


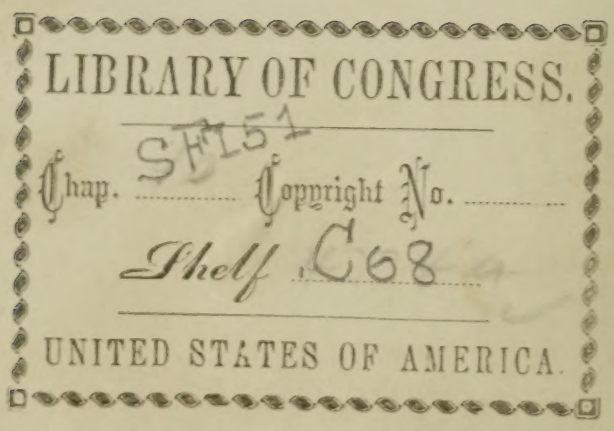

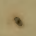



. 


\section{Forty Years' Experience in Veterinary Practice.}

\section{CARE, REARING,}

$\mathrm{AND}$

MEDICAL TREATMENT

\section{DOMESTIC ANIMALS.}

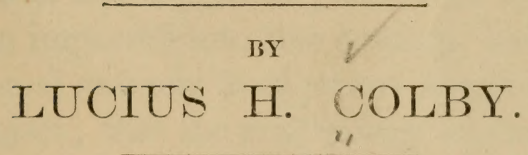

AUBURN :

Wm. J. Moses' Publishing Hougr, 16 Clark St. 
Entered according to Act of Congress, in the year eighteen hundred and seventy-five,

$$
\text { BY LUCIUS H. COLBY, }
$$

in the office of the Librarian of Congress, at Washington, D. C.

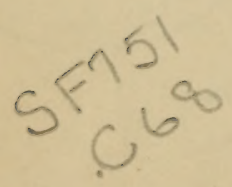




\section{PREFACE.}

This work does not claim to be wholly or even largely original, though it embodies many points in a forty years' experience in the Veterinary practice which have never before appeared in print. In its preparation we have availed ourselves of all valuable and reliable information within our reach, whether to be obtained from books, foreign or domestic, or from the experience and practice of others.

The superior care bestowed in Great Britain upon every class of domestic animals, and the remarkable perfection attained in the culture of horses, sheep and horned cattle, and the consequent high value set upon them when imported into this country, have induced us to give liberal extracts from recent English publications on the subject; that the reader may be enabled to profit by their large experience and great success.

With all these facilities, we have endeavored to compile a work which shall be found generally useful and valuable, and which we know, from personal experience, to be reliable. 



\section{CONTENTS.}

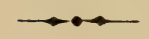

Preface

\section{INTRODUCTORY REMARKS.}

Examination of the Patient. ....................... 19

How to select the Remedies......................... 23

The forms of the Remedies. ......................... 24

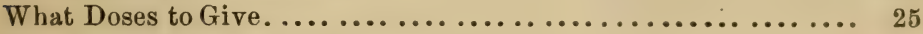

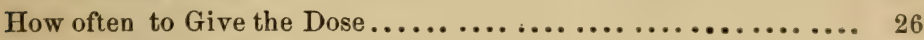

Short Notes on Nursing........................ 27

Chapter I.

DISEASES, ETC., OF THE TEETH.

The Teeth as Tests of Age...................... 29

Irregularities of the Teeth. ..................... 32

Painful and Difficult Teething..................... 33

Caries - Decayed Teeth........................... 34

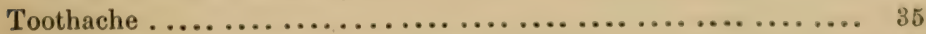

General Symptoms of Diseased Teeth.................. 36 


\section{Chapter II.}

\section{DISEASES OF THE MOUTH.}

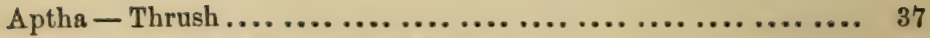

Inflammation of the Mouth and Tongue................ 38

Protrusion of the Tongue. ......................... 40

Injuries about the Mouth, Tongue, etc................. 41

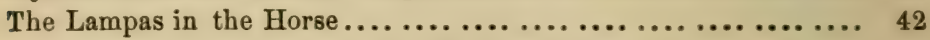

Salivation. ................................. 43

Ranula .................................... 43

Osteo Sarcoma of the Lower Jaw in Oxen................ 43

Gloss Anthrax - Blain......................... 44

Mouth and Foot Disease........................ 47

Mumps ................................... 52

Joint of Lower Jaw Opened....................... 53

Chapter III.

DISEASES OF THE THROAT.

Sore Throat - Inflamed Throat $\ldots \ldots \ldots \ldots \ldots \ldots \ldots \ldots \ldots \ldots, \quad 54$

Inflammation of Guttural Pouches.................. 55

Cases of Sore Throat.......................... 56

Putrid or Malignant Sore Throat.................... 58

Throat Diseases in the Pig........................ 59

\section{Chapter IV.}

\section{DISEASES OF THE GULLET.}

Polypus .................................... 60

Obstruction - Choking .......................... 60

Treatment of Obstruction.......................... 62

Dilatation and Stricture........................ 63

Chapter V.

DISEASES OF THE STOMACH.

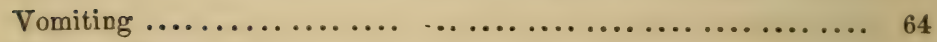

Hove - Hoven. ............................ 65

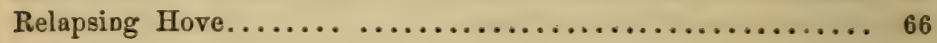




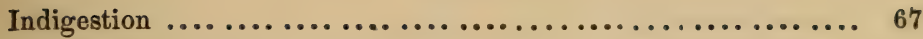

Cases of Indigestion in Horse...................... 68

Cases of Indigestion in Cow. ....................... 69

Stomach Staggers............................. 69

Mad Staggers.................................. 70

Grass Staggers................................. 71

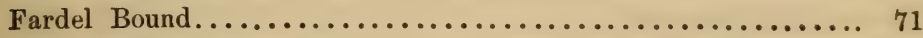

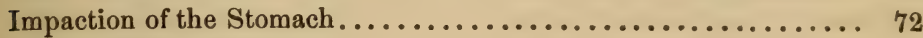

Crammed Stomach............................. 72

Operation of Paunching.......................... 73

Crib Biting and Wind Sucking. .................... 74

Stomach Pain in the Horse $. . . \ldots \ldots \ldots \ldots \ldots \ldots \ldots \ldots \ldots . . . \ldots \ldots$

Loss of Appetite................................. 75

Chapter VI.

DISEASES OF THE BOWELS.

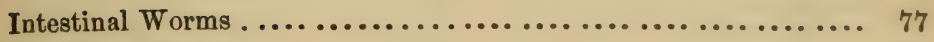

The Horse Bot ................................ 79

Concretions.................................. 81

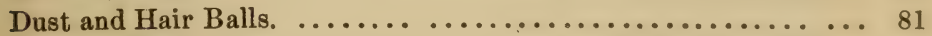

Costiveness.................................. 82

Colic - Gripes................................ 84

Flatulent Colic.................................. 85

Acute Indigestion.................................. 85

Case of Colic .................................. 88

Diarrhœa ........................................ 88

Looseness of the Bowels....................... 88

Case of Diarrhœa in Horse...................... 89

" " "Cow........................ 90

Braxy in Sheep ................................. 92

(Described at page 281, under Carbuncular Diseases.)

Dysentery - Bloody Flux......................... 93

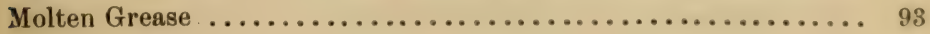

Dysentery in Oxen.............................. 94

Enteritis - Inflammation of the Bowel................ 96

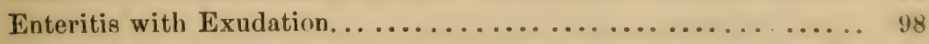

Peritonitis.................................... 99

Chronic Inflammation of the Membrane lining the Abdomen..... 100 
Wood Evil - Moor Ill............................ 102

Twists, etc., of the Bowel......................... 103

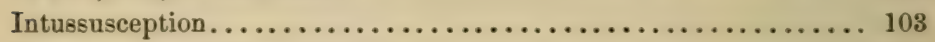

Hemorrhoids - Piles............................ 103

Ascites - Dropsy of the Belly ....................... 104

Protrusion of the Rectum........................... 105

Eversion of the Rectum............................ 105

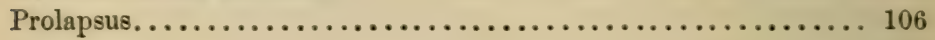

i Chapter VII.

DISEASES OF THE LIVER, SPLEEN, ETC.

Jaundice - The Yellows......................... 107

Hepatitis - Inflammation of the Liver ............... 108

Enlargement of the Liver........................ 109

Flukes in the Liver - Rot ....................... 111

Splenic Apoplexy ............................. 112

Chapter VIII.

DISEASES OF THE KIDNEYS, BLADDER, ETC.

Nephritis - Inflammation of the Kidneys ............. 115

Diabetes Insipidus — Profuse Staling ................. 117

Diabetes Mellitus.............................. 118

Case of Diabetes............................... 119

Scanty Urine. ................................ 119

Retention of Urine............................. 120

Homaturia - Bloody Urine........................ 122

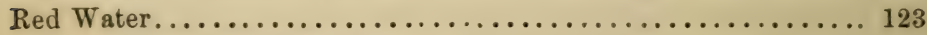

Cystitis - Inflammation of the Bladder................ 124

Calculi — Stones................................ 126

Stones in the Kidney............................. 126

" " $\quad$ Ureter................................. 126

" " Bladder............................. 126

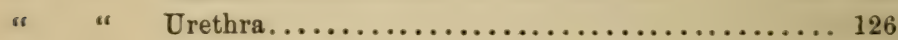

" " Prepuce............................ 126

Dysuria - Difficulty in Urinating. .................. 127

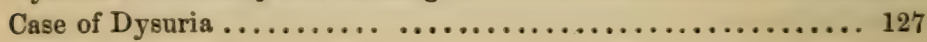


Chapter IX.

\section{DISEASES OF THE GENERATIVE ORGANS.}

Abortion ..................................... 128

Slipping the Calf............................... 128

Precautions against Abortion........................ 130

Premonitory Signs of Abortion, etc. ................. 130

Parturition; Its Treatment ......................... 132

General Treatment to follow Abortion.................. 132

Mechanical Means for Assisting Delivery ............... 136

Flooding after Delivery ......................... 136

Retained Placenta ............................. 137

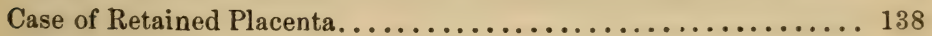

The Early Treatment of Calves. ...................... 138

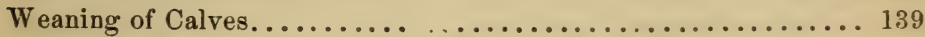

Metritis - Inflammation of the Womb.................. 140

Discharge from the Vagina. ......................... 141

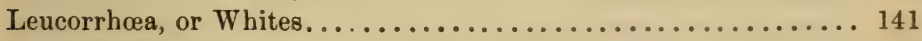

Gonorrhœa, or Clap............................. 141

Inflammation of the Prepuce....................... 142

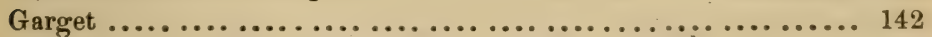

Phytolacea Decandra, Poke Weed..................... 144

Sore Teats.................................. 145

Chapter X.

DISEASES OF THE RESPIRATORY ORGANS.

Bleeding from the Nose .......................... 147

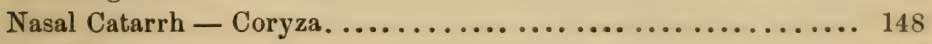

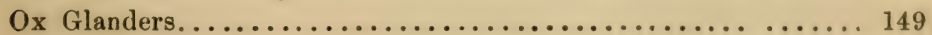

Malignant Coryza............................. 149

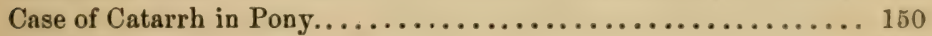

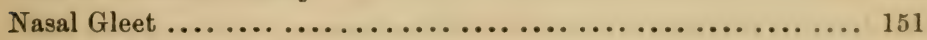

Cases of Nasal Gleet.................................. 152

Collections of Pus in the Nose ....................... 153

Collections of Matter in Guttural Pouches.................. 154

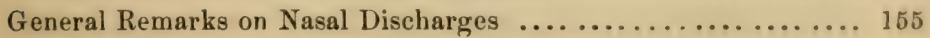

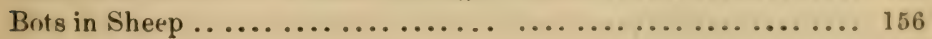

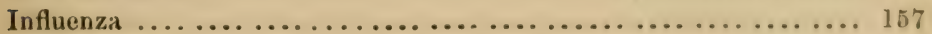

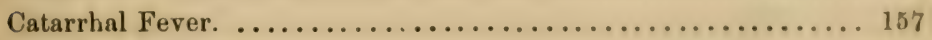

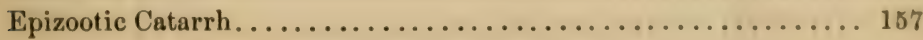


Different Varieties of Influenza..................... 159

Congestion of the Lungs........................... 162

Strangles .................................... 161

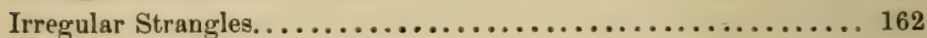

Case of Congestion of the Lungs... .................. 163

Broncho Pneumonia.............................. 164

Bronchitis - Inflammation of the Bronchial Tubes........... 164

Pneumonia - Inflammation of the Lungs ............... 167

Pleura Pneumonia................................ 167

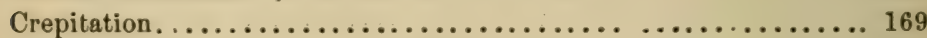

Case of Pneumonia........................... 170

Pleurisy - Inflammation of the Pleura................ 172

Cases Pleurisy................................. 174

Case of Double Pnemonia and Pleurisy.................. 175

“ Pleuro-Pneumonia in Horse..................... 176

“ Typhoid Pneumonia........................ 177

“ Pleuro-Pneumonia.......................... 178

“ Broncho-Pneumonia........................ 179

Abscess ..................................... 181

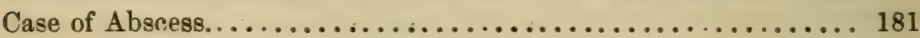

Bleeding from the Lungs ......................... 181

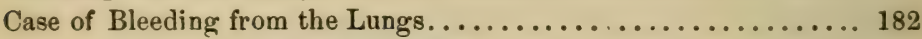

Hydrothorax - Dropsy of the Chest .................. 182

Tapping the Chest................................. 183

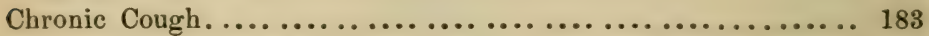

Case of Chronic Cough.............................. 185

Thick Wind - Broken Wind ..................... 186

Roaring, Wheezing, Whistling ..................... 186

Cough in General. .............................. 188

Classification of Coughs ......................... 190

Cough from Dentition............................ 191

Pharyngeal Cough................................ 191

Laryngeal Cough . .............................. 191

Tracheal Cough ................................ 192

Bronchial Cough .............................. 192

Pulmonary Cough ................................ 193

Broken-winded Cough........................... 194

Consumptive Cough. ............................. 197

Chronic Cough................................. 197

Remedies for Cough. ........................... 199 
Pleuro-Pneumonia in Cattle........................ 208

First Stage of Pleuro-Pneumonia in Cattle.............. 210

Second Stage " " "

Third and Last Stage " $\quad$ " $\quad \ldots \ldots \ldots \ldots \ldots \ldots \ldots . \ldots . \ldots . \ldots 212$

Treatment of Pleuro Pneumonia..................... 212

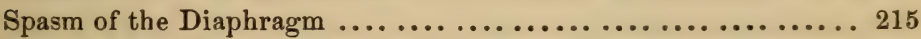

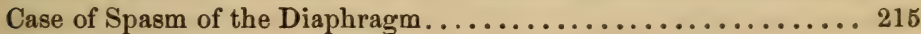

Parasites in the Lungs. .......................... 216

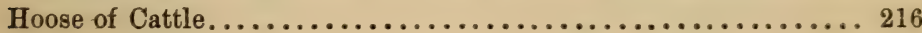

Chapter XI.

DISEASES OF THE HEART.

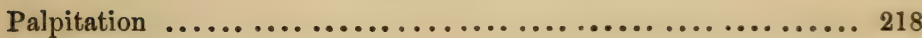

Enlargement of the Heart......................... 219

Dilated Heart................................. 220

Pericarditis ..................................... 220

Inflamed Heart............................... 220

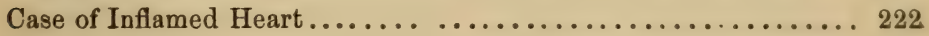

Chapter XII.

DISEASES OF THE NERVOUS SYSTEM.

Rabies - Hydrophobia ........................... 224

Canine Madness, Furious Form.................. 224

" " Sullen Form........................ 225

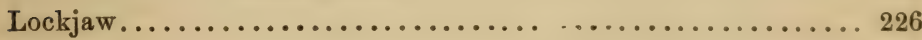

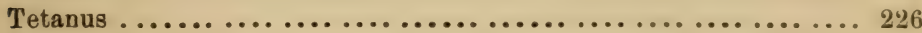

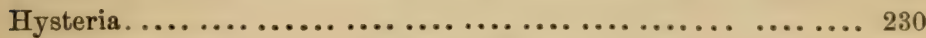

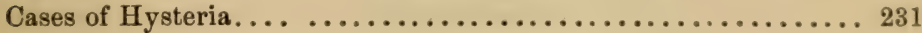

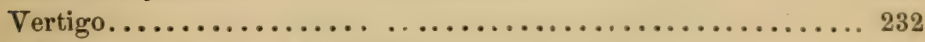

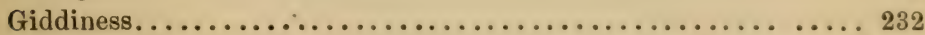

Megrims.................................... 282

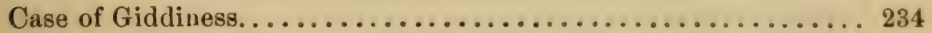

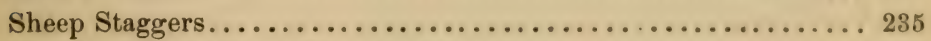

Bladder form of Tape Worm in Sheep................ 235

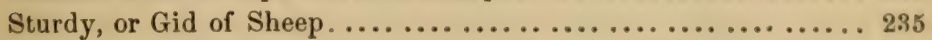

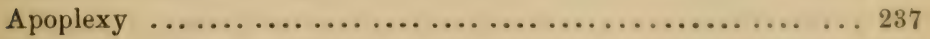

Sunstroke - Coup de Soleil ......................... 238

Staggers ...................................... 239

Mad Staggers.................................. 289 
Brain Fever................................ 240

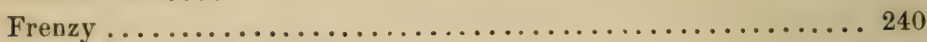

Inflammation of the Brain ........................ 240

Treatment of Giddiness, Apoplexy, Sunstroke, and Mad and Stom-

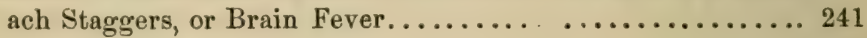

Concussion of the Brain .......................... 245

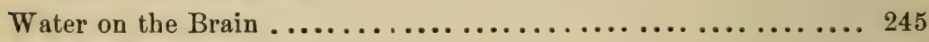

Hydatids of the Brain ............................ 246

Turnsick.................................... 246

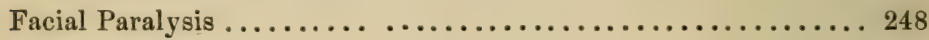

Hemiplegia ................................. 248

Paralysis ................................ 248

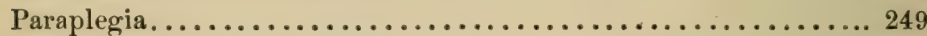

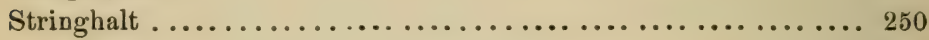

Chapter XIII.

DISEASES OF THE EYE.

Ophthalmia ................................. 251

Inflammation of the mucous membrane of the eye.......... 251

Injuries to the Eye............................ 251

Inversion of the Eyelid............................... 252

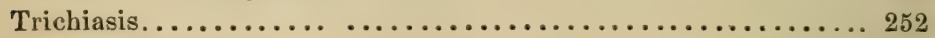

Periodic Ophthalmia............................. 253

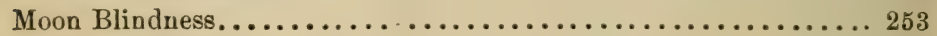

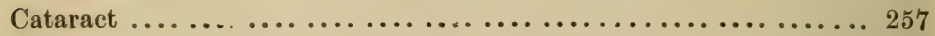

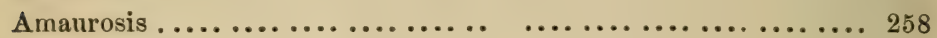

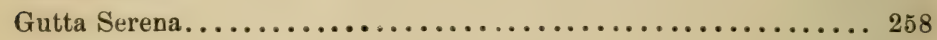

Chapter XIV.

DISEASES OF THE SKIN.

Parasitic Diseases.............................. 260

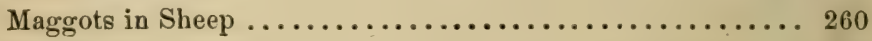

Warbles................................. 261

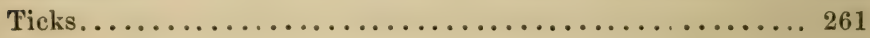

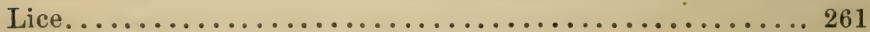

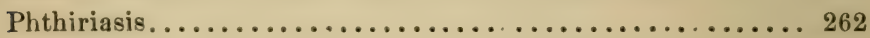

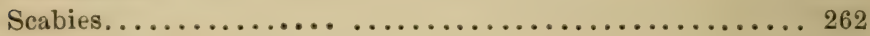

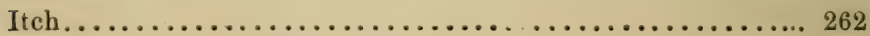

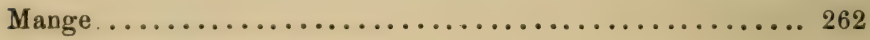


Erythematous Eruptions. ........................ 266

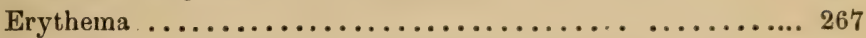

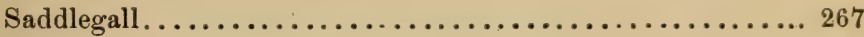

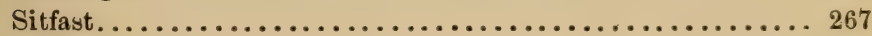

Cracked Heels. .............................. 267

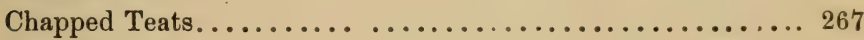

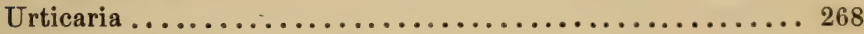

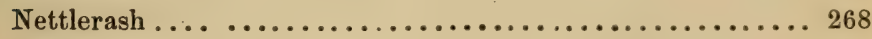

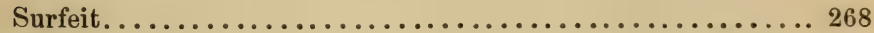

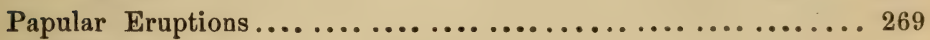

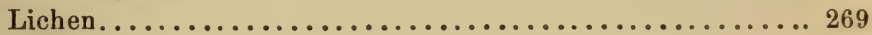

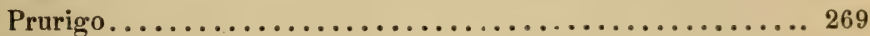

Pustular Eruptions............................ 270

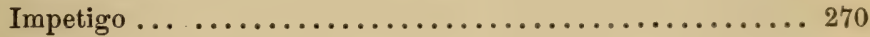

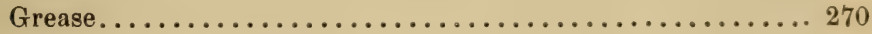

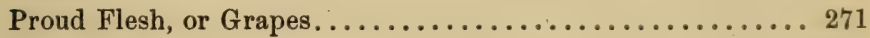

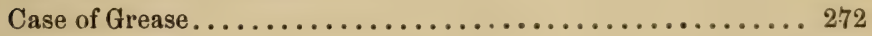

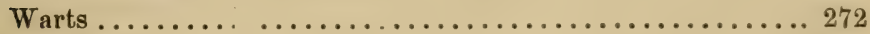

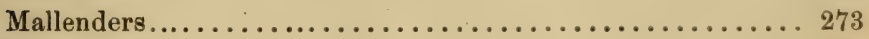

Sallenders............................... 273

Chapter XV.

ERUPTIVE FEVERS.

Erysipelas .................................... 274

Measles..................................... 275

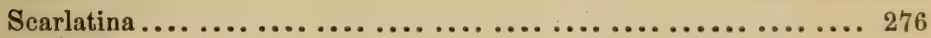

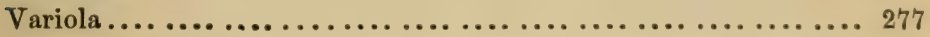

Chapter XVI.

CARBUNCULAR DISEASES.

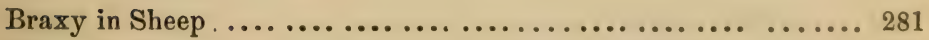

Gloss Anthrax............................... 281

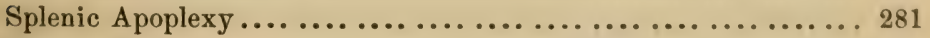

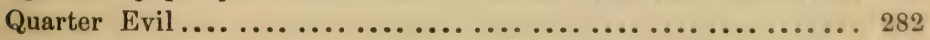

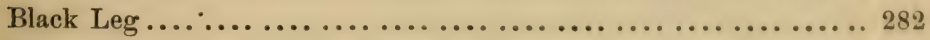

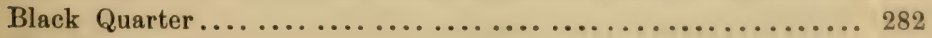

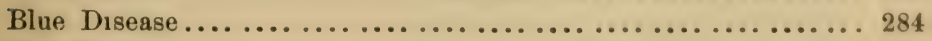

Hog Cholera.................................. 284

Carbuncular Diseases in Pig . ..................... 284 


\section{Chapter XVII.}

\section{WOUNDS AND INJURIES.}

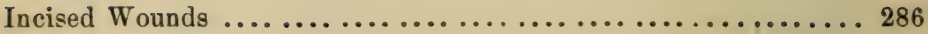

Broken Knee................................. 287

Open Joint. ................................... 289

Poll Evil .................................... 290

Fistulous Withers .............................. 292

Bony Enlargements............................. 292

Nodes ...................................... 292

Bone Spavin .............................. 292

Splint................................ 293

Sidebone................................. 293

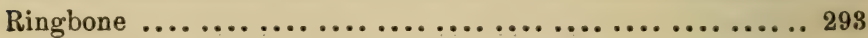

Case of Spavin .................................. 294

" Ringbone............................. 295

Bursal Enlargements............................. 295

Thoroughpin............................ 295

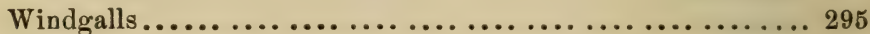

Bog Spavin?............................... 296

Capped Hocks, etc............................. 296

Capped Elbow ................................. 296

Sprains.................................... 297

of the Neck.............................. 297

" Back.............................. 297

" Shoulder............................. 298

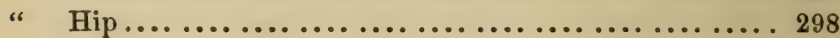

" Stifle............................... 298

" Pelvis Bone............................ 298

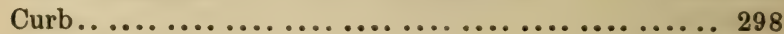

" Back Tendons............................ 299

Broken Down ........................... 299

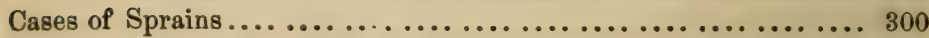

Bruises of the Leg and Foot......................... 302

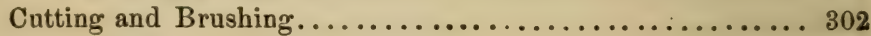

Speedy Cut............................. 302

Overreach ................................ 303

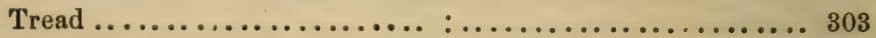

Quittor.................................. 303 
Chapter XVIII.

\section{DISEASES NOT CLASSIFIED.}

Sweeny .................................... 305

Glanders ................................... 306

Farcy ...................................... 308

Treatment of Glanders and Farcy .................... 311

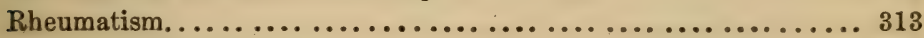

Chronic Rheumatism............................ 314

Lumbago ................................. 314

Case of Rheumatism and Lumbago.................... 315

" " Heart involved................... 316

" " Acute Rheumatism................ 317

Myalgia ....................................... 318

Case of Myalgia ............................. 319

Purpura Hemorrhagica............................ 320

Case of Purpura.............................. 321

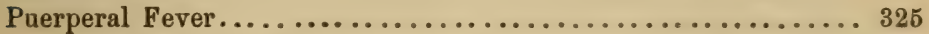

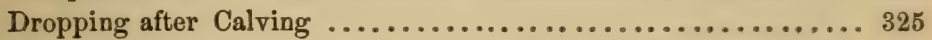

Milk Fever..................................... 325

Treatment of Milk Fever........................... 329

Care of Cows during Milk Fever...................... 331

Case of Milk Fever............................. 332

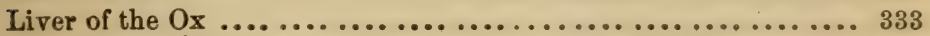

Lymphatitis, or Weed........................... 335

Cellulitis.................................... 337

Foot Rot in Sheep ................................... 337

Ulceration of the Mouth . . . . . . . . . . . . . . . . . 339

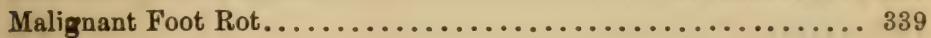

Treatment of Foot Rot. . . . . . . . . . . . . . . . . . . 340

Groggy Lameness................................ 842

Navicular Disease ............................... 342

Picture of Patent Shoe........................ 344

Thrush ................................... 345

Rinderpest. ............................... 347

Cattle Plague of Great Britain...................... 347

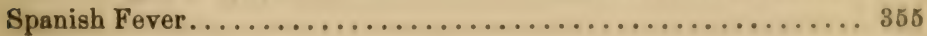

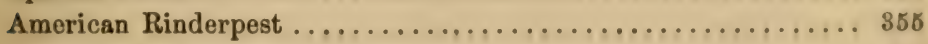

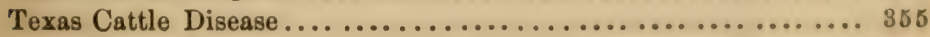




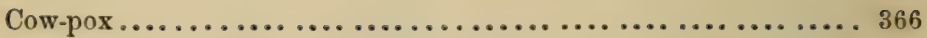

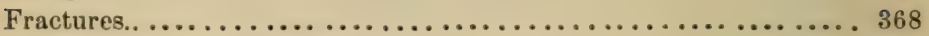

Conformation of the Hock ............................. 375

Spavin - Bone Spavin ......................... 377

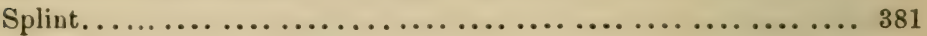

Ringbone................................... 386

Contraction. . . . . .............................. 388

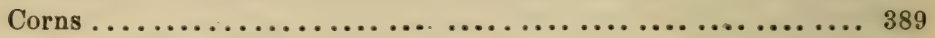

Synovial Membranes............................ 390

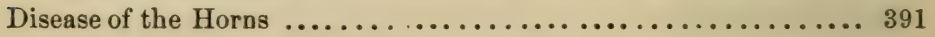

Chapter XiX.

REARING OF SHEEP, SWINE AND POULTRY.

Various Breeds of Sheep, and their productive merits......... 393

The Wool and its Peculiarities . ....................... 399

The General Management of Sheep ................. 401

Parturition of Sheep............................ 404

Early Treatment of Lambs......................... 407

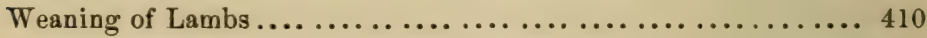

Use of Ewe's Milk.............................. 412

Use of Sheep's Skin .......................... 413

Teeth, as indications of Age in Sheep ................. 413

Shearing ................................. 416

Breeding, Rearing, Weaning, etc., of Pigs............... 416

Preparatory Treatment, and Killing of Pigs.............. 418

Poultry ................................... 419

Breeding, Rearing, and General Management of Poultry ....... 423

Feeding of Poultry for Market or Slaughter.............. 433

Ducks and Geese .............................. 435

Of Turkeys............................... 440

Diseases Prevalent among Poultry .................. 442

\section{APPENDIX.}

Table of the Medicines Prescribed in this Work ........... 446

List of External Applications...................... 448

Directions for Compounding Medicines................ 453

Recipes.................................... 454

Glossary................................ 460

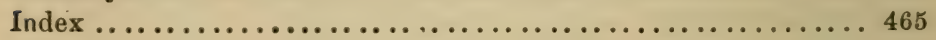




\section{HANDY BOOK}

OF

\section{VETERINARY HOMCOPATHY,}

WITH

FORTY YEARS' EXPERIENCE. 



\section{INTRODUCTORY REMARKS.}

In using this work as a guide to the homøopathic treatment of the diseases of our chief domesticated animals, the reader is particularly requested to "mark, learn, and inwardly digest" the following observations:

The rules respecting-

How to select the remedy;

What dose to give;

How often to give the dose;

must be specially studied, and referred to, after having settled what the disease is, and what remedy is indi. cated.

\section{Examination of the Patient.}

This is the first thing to do when an animal is ill. The symptoms which it presents must be minutely inquired into, for two purposes : firstly, to determine the seat and nature of the disease ; and secondly, to obtain indications for the selection of the right remedy. Attention to the following points will be of service:

\section{1.-The Organs of Circulation.}

(1.) The heart should be examined by the hand, ear, or stethoscope being placed on the left side, behind the 
elbow-joint, to ascertain the force and rhythm of its action, and the character of its sounds, whether natural, or unnatural.

(2.) Important information is gained by learning the state of the pulse,-whether regular or irregular, soft or hard, frequent or slow, \&c.

The horse's pulse is best felt where an artery passes over the edge of the lower jaw, about three inches from the angle of that bone; it may also be felt at the inner side of the leg, near the elbow-joint.

In cows the pulse may be felt, as in the horse, at the jaw, or fore leg. A convenient place, in some cases of illness, is over the fore fetlock, exactly in front, in a line with the cleft of the foot.

In smaller animals, the pulse may be felt on the inside of the arm, or thigh.

The beats per minute of the pulse are, on an average, in health and adult life, in the horse, 36 ; in the ass and mule, 46 ; in the ox, 45 to 50 ; in sheep, goat, and pig, 70 ; in dog, 90 .

It must not be forgotten, however, that the pulse varies somewhat from the healthy standard in all animals, under the influence of various circumstances, including age, size, temperament, breed, mode of feeding, domestication, \&c.

\section{2.-The Respiratory System.}

(1.) Ascertain if the nose is hot or cold; dry or moist, covered or not with hardened mucus ; if there is sneezing, or offensive smell, or indications of polypoid growths or foreign bodies in the nostrils ; if there is discharge-its 
character, whether watery, gluey, mattery, or bloody; if ulcers can be seen on the nasal membrane.

(2.) The character of the breathing,-frequent, difficult, painful, labored, \&c.

In a healthy animal, in complete rest, the frequency of breathing is nearly in the proportion of one act of breathing to four beats of the pulse. In the horse, there are, taking an average, 10 acts of respiration in the minute -15 in the ox -15 in sheep-20 in the dog-in smaller animals, higher.

(3.) If the expectoration, when there is any, is scanty or abundant; mucus or pus, or both ; if expelled easily, or with the aid of vomiting.

(4.) If the cough is rare or frequent, painful, hoarse, croupy, barking, dry or moist, recent or chronic.

(5.) If both sides of the chest expand equally during breathing, or if one side expands more than the other; if pressure causes pain, flinching, and grunting.

(6.) If the sound is clear or dull when the lungs are percussed; if dull, noting the position.

(7.) If, on listening to the chest, the sounds attendant on respiration are natural or morbid ; if the latter, ascertaining their character and marking their site.

\section{3.-The Digestive System.}

(1.) If the teeth are all, or only in part cut; rotten, broken, worn down, loose, black, incrusted with tartar, irregular, \&c.

(2.) If the tongue is bitten, swollen, inflamed, cut, or paralyzed on one side; if furred; moist or dry ; or altered in color; or covered with vesicles, or ulcers. 
(3.) If the mouth is hot and dry, or cool and moist; if the breath is offensive, or not; if the mucous membrane is inflamed on the gums, in company with the peculiar odor of mercurial poisoning and salivation.

(4.) If swallowing is difficult or painful, or attended with choking; examining the throat internally for bones, polypus, \&c., and externally for enlarged glands, \&c., bronchocele, \&c.

(5.) If the stomach is disordered, as indicated by the appetite, thirst, vomiting, \&c. ; the vomit being specially observed as to appearance and smell, and, if necessary, analyzed in cases of suspected poisoning.

(6.) The size of the belly; whether hard, or soft, or painful; dull or clear when percussed, or fluctuating when tapped; containing tumors or foreign bodies; if there is diarrhœea, dysentery, constipation, piles; the character of the expelled excretions, as to color, consis. tence, quantity; the existence of pregnancy.

(7.) The size, position, and feel of the liver; if there are indications of enlargement, or of jaundice.

\section{4.-Genito-Urinary System.}

(1.) The functions of the uterus; discharges from, or tumors connected with it.

(2.) The state of the vagina; if the seat of polypus, cancer, ulceration, displacement, discharge, or injury.

(3.) If the milk-glands or teats are tender, swollen, hard, cancerous, inflamed, ulcerated, or pocked, \&c.

(4.) If the penis or prepuce is inflamed or ulcerated ; or the seat of fungous growths, stones, \&c.

(5.) Note the way in which urine is voided, and whether there is, or is not, a discharge of blood. 


\section{5.-The Intggumentary System.}

(1.) The skin, whether blotched, or mangy, or otherwise diseased ; if the disease is partial or universal ; the seat of morbid growth, dropsy, \&c.; if hot or cold.

(2.) The state of the feet, nails, and pads.

\section{6.-The Nervous System.}

(1.) The shape and size of the head; whether the scalp is injured, or the skull fractured.

(2.) Insensibility, or profound coma.

(3.) If the special senses of sight, hearing, \&c., are diminished or lost; noting at the same time the condition and color of the eye.

(4.) If there are fits, convulsions, trembling, rigidity, palsy, or any peculiarity of movement when walking, such as avoiding, or rolling over obstacles in the way, or turning round and round.

It would be easy to enlarge the objective symptoms of disease, but the above may prove sufficient as fingerposts in aid of practical observation and accurate diagnosis.

\section{How to Select the Remedies.}

The symptoms having been ascertained, and for the sake of reference, instruction, or publication, noted down from day to day, the next step is to select the proper 
remedy for the disease. Under the "treatment" of each malady, a few remedies are given, with eertain symptoms attached to each. That medicine, or those medicines, are to be given, whose symptoms correspond the most exactly with those of the disease. For instance, if the disease should be influenza, Aconite is to be given when the symptoms recorded in connection with it, are present in the patient; and Belladonna when the symptoms recorded after it, are present. Should the symptoms of both these medicines be present in the same case, then both medicines should be given, not mixed, but separately and in alternation. These instructions are applicable to every other disease. I may here remark that all the appropriate remedies are not mentioned, but only those that are generally useful in ordinary cases.

\section{The Remedies.}

: The medicines prescribed in this work, and used in my practice, are internal and external.

The internal are in two forms :-

(1.) Tinctures, or alcoholic solutions of the remedy, varying in the dilution, usually the first decimal.

(2.) Triturations, in which the crude drug is minutely subdivided, by being rubbed up with a non-medicinal substance in certain fixed proportions. These are used less than tinctures.

I sometimes give Pilules, which are non-medicinal vehicles, saturated with a certain dilution of any given 
medicine. They are specially suitable for small dogs, when it is necessary to give medicine, either from the dog refusing, or being unable, to take it.

For the lists of the internal remedies and external applications, refer to the Appendix.

\section{What Dose to Give.}

The following directions on this point apply to all the internal medicines mentioned in this work, and should be followed out, except when the dose is specially stated under the "treatment."

The average doses for ordinary attacks of the diseases mentioned in this treatise are stated, in the body of this work, as " 20 for horses and oxen; 10 for sheep and pigs; 4 to 10 for dogs." The following table will give at a glance the average dose for each of the animals named :-
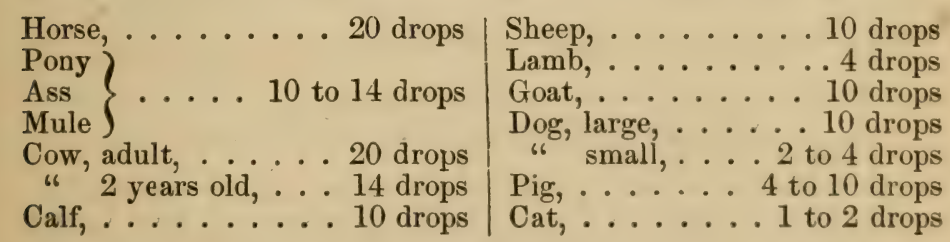

In order to do away with the trouble and inconvenience of having to drop each dose when it is required, the best plan is to mix enough for one or two days. For instance, in the case of the horse and cow, drop 120 drops of the tincture into a perfectly clean bottle, add 4 wineglassfuls of pure cold water, and cork up. Of this mix- 
ture give a wine-glassful, by means of a clean horn, ${ }^{*}$ as frequently as may be required. In the case of smaller animals, mix the dose, as stated above for each, with a teaspoonful of water ; thus, for a medium-sized dog, add 24 drops of tincture to 6 teaspoonfuls of water, and give a teaspoonful when required. Each wine-glassful and each teaspoonful of the two mixtures thus made, will, of course, contain 20 drops and 4 drops respectively. Two medicines must never be mixed together. If two medicines are being given to the same horse or cow, two horns should be used-one for each medicine.

In the sleepy stage of milk fever, instead of mixing with a wine-glassful of water, I use only a teaspoonful,because in such cases swallowing is difficult or impossible, and a small quantity of fluid is more readily absorbed by the mouth than a large quantity.

The triturations-the doses of which are stated in the body of the work-are given to horses in a handful of bran mash; or, when it can be managed, in all animals, dry on the tongue.

\section{How often to Give the Dose.}

In acute cases, such as inflammation of the lungs, or in such as are attended with pain, or are evidently attended with great danger unless speedily checked, the medicine, or medicines, should be given frequently,every quarter of an hour, half-hour, hour, or every two hours, according to the violence of the symptoms. When

\footnotetext{
* Small horns, specially made, can be got from most homœopathic chemists.
} 
the disease is within the remedial power of medicine, and the remedy is adapted to the disease, improvement will set in, in a majority of cases, after a very few doses. Then it follows that the medicine, or medicines, must be given less frequently than before. When two medicines are required to meet all the symptoms, each dose of them is to be given alternately; for instance, if Aconite and Bryonia are both indicated, and if the severity of the disease necessitates their administration every hour, $A$. is to be given, say at the even hours, $2,4,6$, and $B$. at the odd hours, $3,5,7$, etc. And so with the others.

In old-standing cases, or in chronic diseases, the medicine may be given two, three or four times a day, according to circumstances.

\section{Short Notes on Nursing.}

When an animal is ill, it should, if possible, be placed by itself in a clean, well-ventilated, and moderatelywarmed box, or stall. This is especially necessary in the case of catching diseases, as well as when noises are injurious, as in lock-jaw, or when the horse rolls about violently, as in colic. The excretions and soiled bedding should be removed as often as may be necessary. For horses, plenty of straw should be laid on the floor, particularly in colic, \&c. Fresh hay, or clean wood shavings, form the best bed for sick dogs; but pet dogs have usually a more elegant couch.

When the skin is cold, and the hair rough and staring. 
sufficient clothing should be put on to ensure a comfortable degree of warmth. The legs of the horse may also be swathed in flannel bandages, which should be removed occasionally, and, after hand-rubbing the legs, put on again.

In diet, it is generally the safest and best course to follow the cravings of appetite. In acute cases, the appetite is impaired, or absent, and the animal will eat little or nothing, even when food is within his reach. As improvement sets in, in many cases of diseases, the appetite is more or less keen, and the patient is apt to gorge himself, and do himself serious injury. The rule is to give small quantities freqnently, rather than a large quantity at long intervals. Hay is good at all times given in bits, provided it be good and clean. Cold water, or cold hay tea, in small, frequent sips, should not be forgotten. Bran mashes are good during most illnesses, and boiled linseed in sore throat and colds. Carrots and boiled oats, or barley, are always relished during recovery from any illness, as well as green food, when it can be got.

Occasionally it is necessary, when the animal is unable to eat, to give food by drench or injection.

Rough usage is to be avoided, both for humanity's sake and for medical reasons. In tetanus, and some other diseases, an angry word, or rough handling, causes a great increase of suffering. 


\section{CHAPTER I.}

\section{DISEASES, ETC., OF THE TEETH.}

\section{1.-The Teeth as Tests of Age.}

The process of dentition from early to advanced life, especially with respect to the order in which the different sets of teeth make their appearance through the gum, and the striking changes in their shape and marks, has been satisfactorily elucidated by several able investigators. The information derived from an examination of the mouth furnishes reliable grounds, as a rule, for giving a trustworthy opinion on the animal's age; and, in the case of the horse, an experienced man can speak with absolute exactness, except when the horse is aged.

\section{IN HORSES.}

In the foal, the two middle incisors, or front teeth of both jaws, are cut at birth, or they appear shortly afterwards. These are presently followed by three molars, or grinders, on each side of both jaws, and at the sixth week, by two other incisors in each jaw. At about the ninth month, the corner incisors are cut. When a year old the horse has, therefore, 12 molars and 12 
incisors. From the first to the second year, the teeth already cut are in full use and wear, and two more molars on each side of both jaws appear in succession. At two years there are, therefore, 12 incisors and 20 molars, all temporary teeth. Between the second and third year these teeth begin to fall out and give way to the permanent set, which appear in the same order as the first set. The two middle incisors in the upper and lower jaw are the first to be replaced by stronger and larger permanent incisors, marked on the cutting surface by a deep, dark hollow ; from three to four, the next incisors above and below are replaced; and from four to five, the corner incisors-making in all 16 permanent incisors. By four and a half, the temporary molars have gradually given way to corresponding permanent molars, and the sixth and last molar, a permanent tooth, appears, as well as the four tusks, or tushes, of the horse. At this period the full number of teeth are cut, viz., 44 in the horse, 40 in the mare, and at five they are all in full wear, and undergoing, especially the incisors, those changes which furnish the best evidence of the animal's age. At six the lower middle incisors are without mark; at seven the same change is found in the other incisors; and at eight, all the black marks are worn out, even in the corner incisors. At a later period, the incisors become triangular on their cutting surface, and stand out straighter from the jaws. The marks are retained longer on the upper incisors, but they are less reliable than those on the lower as indications of age. The tushes, too, at first small and sharp, gradually, as age advances, become longer, blunter and yellower. In exceptional cases, one may meet with an old horse having a young mouth, as far as the shape 
and marks of the incisors are concerned, but the changes above described generally hold good. It is important not to mistake the temporary for the permanent teeth, otherwise a two-year-old may pass for a five-year-old, with a "green" buyer. The permanent teeth are larger and darker, and have a furrow down the centre on the outer surface.

\section{IN OXEN.}

Oxen have eight incisors in the lower jaw, and in the upper no teeth, but instead, a "pad" of elastic tissue; and 24 molars. In early life, the age is ascertained by the times when the temporary are succeeded by the permanent teeth, and later, by changes in the teeth themselves, which become narrower, blunter and stumpy. In addition, count three for the space between the top ring on the ox's horn and the top, and one for each of the other rings. Professor Simonds gives the following table of the successive changes in the teeth of this animal :-

\begin{tabular}{|c|c|c|c|}
\hline \multicolumn{2}{|c|}{$\begin{array}{l}\text { TABLE OF EARLY AVERAGE- } \\
\text { IMPROVED BREEDS. }\end{array}$} & \multicolumn{2}{|c|}{$\begin{array}{l}\text { TABLE OF LATE AVERAGE- } \\
\text { IMPROVED BREEDS. }\end{array}$} \\
\hline Years. Months. & No. of Teeth. & Years. Months. & No. of Teeth. \\
\hline & $\begin{array}{l}2 \text { permanent } \\
\text { incisors. }\end{array}$ & $\begin{array}{llll}2 & \ldots & 3 & \ldots\end{array}$ & $\begin{array}{l}2 \text { permanent } \\
\text { incisors. }\end{array}$ \\
\hline .. 3 & $4 \quad$ “ & .. 9 & $4 \quad 6$ \\
\hline .. 8 & 6 & $\begin{array}{lll}3 & \ldots & 3\end{array}$ & 6 \\
\hline $3 \ldots 3$ & 8 & $3 \ldots 9$ & \\
\hline
\end{tabular}

\section{IN SHEEP.}

In the sheep, also, the age is determined partly by the shedding of the temporary for the permanent teeth, and 
partly by the wearing appearance of the teeth themselves. On an average, at one year the central pair of temporary incisors are replaced by the permanent-at one and a half, the second pair-at two and a half, the third-and at three, the fourth pair.

\section{IN PIGS.}

At the first month, there are four incisors, and three temporary molars on each side of both upper and lower jaw. At three months, two more incisors appear in each jaw, and this completes the temporary set. In some, at about the sixth month, false tushes appear in both jaws in front of the molars. At the sixth month, the fourth molar is through. At nine months, the permanent corner incisors and permanent tushes are cut, and the fifth molar on each side. At twelve months, the middle incisors are replaced, as well as the temporary molars. At, or about the eighteenth month, the process is completed in the pig by the appearance of the side incisors, and sixth and last molar.

\section{2.-Irregularities of the Teeth.}

One of the most common irregularities occurs during growth, when one incisor projects from the jaw instead of being side by side with the others. The term "buck" tooth is applied to this condition. The remedy is removal. In some cases a temporary tooth has to be drawn in order to make room for the proper placing of a per- 
manent one. A supernumerary tooth--"wolf's" tooth - sometimes appears in front of the molar set in either jaw; when it interferes with chewing, it should be extracted. An outgrowth of bony substance from a tooth has been met with, and should be chipped off if it cause any inconvenience. The molars are often irregular. One, instead of coming through the ridge of the gum, may protrude through the roof of the mouth, and when long, injure the tongue and prevent eating and chewing. From irregular wearing of the surface of the molars, the edges of these teeth become sharp and jagged, causing wounds of the inside of the cheek or tongue, and interfering with chewing. These sharp edges should be rasped down. Occasionally, the excessive growth of a molar leads to injury of the upper jaw, followed by inflammation and ulceration, with offensive discharge from the nose, swelling of the face, and impaired chewing. Such a tooth should be rasped down or drawn.

In rasping, or dratwing the teeth of animals, instruments made for the purpose must be used, and a veterinary surgeon called in to handle them.

\section{3.-Painful and Difficult Teething.}

\section{IN HORSES, OXEN, \&c.}

In some horses the process of teething gives rise generally to much disturbance, especially in the case of the tushes. There is a certain amount of feverishness, attended with refusal to eat, difficulty and pain in chewing, disorder of the bowels, and even colic, passage of 
uncrushed oats, irritating cough, loss of flesh and condition, \&c. The gum is found to be hot, painful and swollen.

\section{Treatment.}

Belladonna is the best remedy when the animal is feverish and suffers much pain, and when the gums are tender to the touch.

Calcarea is of use when the teeth are slow in appearing, as the result of constitutional weakness.

Doses.-20 drops of these medicines, night and morning, for the horse and cow; 10 drops for sheep and pig.

There are cases where it is necessary at once to remove the urgent symptoms by freeing the tooth, which is done by cutting through the gum cross-wise over the tooth. Also, pull out loose or superfluous teeth.

\section{4.-Caries-Decayed 'Teeth. IN HORSES.}

The incisors never, the molars frequently are rotten. This is indicated by a peculiarly offensive smell issuing from the mouth, by slavering, by swelling, redness and pain of the gum around the diseased tooth, by a black spot or cavity in the tooth, and by accumulation of foul food in its vicinity. There are fits of toothache, especially when it is the third molar that is affected. When the three last molars are decayed, and the gum inflames, the matter resulting therefrom makes its way into the maxillary sinuses, and thence flows through the nose, giving rise to much local disturbance, general irritative 
fever, most offensive stench, and presenting a condition which has often been mistaken for glanders. In some cases of decayed molars, in addition to other symptoms, one eye is inflamed and watery. Sometimes the abscess bursts on the surface, and then we have to deal with that troublesome state known as Fistula of the face. A diseased lower molar may set up inflammation of the lower jaw, with enlargement of the bone, and mattery discharge -a condition which I have seen more than once, and cured by the extraction of the offending tooth, followed by general and local treatment. The teeth should be carefully examined in all cases of pain and swelling of the bones of the face and jaws, particularly if accompanied by nasal discharge and imperfect chewing.

\section{IN OTHER ANIMALS.}

The symptoms are those given above, and the same general treatment should be carried out.

\section{Treatment.}

It is essential at once to extract diseased teeth, and if the contiguous bone is diseased to give Silicea, in 20 drop doses three times a day. If matter has discharged on the skin, keep the part thoroughly clean by washing with warm water, and treat locally for fistula, as directed in the remarks on that subject.

\section{5.-Toothache.}

\section{IN HORSES.}

When a horse shows from his conduct that he is suffering pain, and when he refuses his food, or cannot chew 
it properly, hangs his head, and is worse in these respects after drinking cold water, he may have a rotten tooth, or be suffering from some other unnatural condition of the mouth.

\section{IN OXEN, \&c.}

The symptoms are much the same. In all animals the only treatment is to pull out the diseased teeth.

\section{General Symptoms of Diseased Teeth.}

Animals suffering from diseased or irregular teeth present some, or all of the following symptoms:-The food is not chewed thoroughly, but after having been partly ground down, is thrown out. Horses "quid" their hay and bolt their corn, which is seen in the dung uncrushed. The result is, that partly from constant pain and partly from loss of nourishment, the animal gets dull, thin and hide-bound, and then is apt to suffer from colic, or some other disease incited by its low condition. Some of the consequences of diseased teeth to the parts adjacent, in setting up irritation, inflammation and nasal discharge have been already referred to. In caries, there is a most disgusting smell issuing from the mouth, attended with increased flow of saliva, indications of acute pain, and swelling and redness of the gum around the diseased tooth. Whenever these symptoms are met with, a careful examination of the mouth should not be neglected. 


\section{CHAPTER II.}

\section{DISEASES OF THE MOUTH.}

\section{1.-Aphtha-Thrush.}

\section{IN HORSES.}

Thrush is occasionally met with in the horse, although less frequently than in other animals. It consists in an eruption of small vesicles on the mucous membrane lining the mouth. When the fluid in these vesicles is discharged, small ulcers are observed, and this ulcerated condition may be somewhat widely spread, from the small ulcers running together. The lining of the mouth peels off, leaving below a tender surface, which prevents the animal from eating as usual. On examining the mouth, these appearances are observed on the tongue, lips, inside of the cheeks and gums. In the horse, thrush is sometimes accompanied by strangles. It arises in all cases either from the local irritant action of improper food, or acrid plants, or as'the result of constitutional disorder.

\section{IN OXEN.}

In oxen, thrush is often observed. The symptoms are the same as those found in the horse, with the addi- 
tion of a more profuse discharge of stringy slaver from the mouth. Thrush is not "foot and mouth disease:" it is not contagious, does not spread like an epidemic (epizootic in the lower animals), and is not associated with disease of the teats and feet; by these differences simple thrush may be known from the more serious murrain. It should also be distinguished from the rinderpest, in which disease there is a peculiar thrush-like condition of the mouth and lips. Oxen do not chew the cud as usual.

\section{IN SHEEP.}

Sheep, also, are often attacked with thrush, the most frequent cause being, in them as in oxen, eating acrid irritating plants. It is found associated with foot-rot in some cases. For the symptoms, read the above description.

\section{Treatment.}

In all cases begin with Kali Chloricum.

If, after giving this remedy for a few days, no improvement is manifest, substitute Mercurius.

Doses. - 20 drops for horse and cow, thrice a day; 10 drops for pig and sheep.

\section{2.-Inflammation of the Mouth and Tongue. IN HORSES.}

It has been known to follow the internal administration of turpensine in too large doses, and it occasionally 
arises from other more obscure causes. The whole of the mouth is more or less affected with redness, pain and swelling, and throughout the progress of the disease there is much febrile excitement. In course of time, the lining membrane of the mouth peels off, and the parts are left raw and sore, and extensive ulceration and even gangrene may result. There is copious discharge of mucus. In some cases the tongue is more severely inflamed than the other parts of the mouth-then it is swollen and hangs out, and in bad cases, matter forms in it, or it is left hard, stiff and enlarged. When the tongue is much swollen, swallowing is difficult, and the breathing so impeded as to threaten choking. One of the most frequent causes of inflamed tongue in the horse is injuries resulting from violence in giving balls, or from the animal biting his tongue.

\section{IN OXEN.}

The causes are the same as in the horse, and it may also follow the administration of hartshorn given to cattle suffering from hove. The symptoms and treatment of this disease in the ox, and other animals, are those given above and below.

\section{Treatment.}

Aconite should be given at first when the animal is feverish, the mouth dry and red, and the tongue swollen and painful.

Arnica is indicated when the inflammation has resulted from injuries. Arnica lotion* may also be applied locally.

\footnotetext{
* See list of local applications.
} 
Mercurius may be given with good results when, as a result of acute, unchecked inflammation, the tongue is hard and much swollen; also, when there is great dribbling, difficulty of swallowing, and redness and rawness of the mouth generally.

Hepar Sulphuris may be required when matter is forming in the tongue.

Arsenicum is the best remedy when gangrene threatens.

Doses. -20 drops for horse and cow, every 2 or 3 hours ; 10 for sheep and pig.

In addition, if suffocation be imminent on account of the great swelling of the tongue, and if matter have formed, incisions should be made into the tongue. Plenty of cold water should be allowed, together with gruel, linseed tea, \&c., and if necessary, thin fluid foods should be given by drench.

t.

\section{3.-Protrusion of the Tongue.}

\section{IN HORSES, OXEN, \&c.}

This is a symptom of paralysis, weakness of the muscles of the tongue, and of injuries. Paralysis of the lips on one side sometimes co-exists, resulting from local injury to the nerves distributed to the parts. The lower lip then hangs down, and food is picked up with difficulty ; there is also more or less slavering. Of course, the tongue is very liable to be severely injured when it falls between the front teeth, and repeated injuries of this kind may result in troublesome wounds or ulcers. 


\section{Treatment.}

When arising from injuries, give Arnica.

When a symptom of paralysis, the most likely medi. cine to do good is Nux Vomica.

The last medicine failing to improve after a month's course, try Belladonna.

Dosfs.-20 drops, night and morning, for horse and cow ; 10 for sheep and pigs.

Generally, the tongue must be shortened, otherwise it is continually falling between the teeth and getting injured.

\section{4.-Injuries about the Mouth, Tongue, \&c.}

\section{IN HORSES.}

The tongue may be injured by the forcible and awkward administration of balls and drinks, and the fringe of membrane which connects the under surface of the tongue with the floor of the mouth may be torn. The lower jaw and angle of the lips are sometimes hurt, and even a portion of the tongue severely crushed, by bits, or rough usage in riding or driving. Rough, irregular, too long teeth may tear the tongue, or bruise the cheeks and palate. Needles, thorns, sharp bones may stick in the tongue, or mouth. In cart horses, particularly, by the absurd use of bearing-reins, the angle of the mouth 
is bruised, and an abscess forms, which may break in the inside or outside.

The tongue is also liable to injury when it protrudes from the mouth, as has been already mentioned above.

\section{Treatment.}

In all cases apply Arnica lotion* three or four times a day, and Calendula lotion* for open wounds, cuts, \&c. Remove foreign bodies. When ulcers form, give Hepar Sulph.

Doses. -20 drops for horse and oxen; 10 for sheep and pigs.

\section{5.-The Lampas in the Horse.}

By this name is called a swelling, more or less painful, of the bars of the roof of the mouth, behind the front teeth, generally on a level with the teeth's surface, sometimes projecting lower. It is not a specific disease, but a symptom connected with the shedding of the teeth in young horses, or with disordered stomach. It interferes with feeding and chewing, causes the horse to be off his feed, and is usually attended with some degree of feverishness.

\section{Treatment.}

Let it alone, or treat as for "Difficult Teething," (page 34 ), or "Indigestion," (which see) according to the symptoms "present. If any one recommends "burning," ask to have the same measure tried on the roof of his mouth.

* See list of local applications. 


\section{6.-Salivation.}

IN HORSES, OXEN, SHEEP, \&c.

Excessive dribbling of slaver from the mouth has already been alluded to, as a prominent symptom in inflammatory diseases of the mouth, \&c. It has been observed from eating grass containing mustard, and from the use of mercury, internally or externally.

\section{Treatment.}

Remove the cause, frequently inject cold water into the mouth, and when it does not arise from mercury, give Mercurius; when it does, Hepar Sulph.

1)oses.-20 drops for horse and ox; 10 for sheep and pigs - one dose three times a day.

\section{7.-Ranula.}

Ranula consists in obstruction and distension of the duct of one of the glands which secretes saliva, under the tongue, on one or on both sides. When large, it is very troublesome.

\section{Treatment.}

Open with the lancet. Give 20 drops of Mercurius, three times a day. If necessary, dress the wound with Calendula lotion.*

\section{8.-Osteo-Sarcoma of the Lower Jaw in Oxen.}

This is the scarcely accurate name of a disense affecting the lower jaw, sometimes the ribs also, of young

* See list of local applications. 
cattle, and of steers oftener than bulls. The favorite seat is that part of the lower jaw where the second and third molars are attached. Occasionally, but rarely, the upper jaw is affected. It begins in the form of a small, painful swelling, which grows either rapidly or slowly, and is attended with gradually increasing pain. The bone becomes seriously involved, the teeth become loose or rot, and fall out, whilst the pain and impeded chewing induce wasting. It is very common in some parts of Italy, especially in low bred cattle.

\section{Treatment.}

The only likely means of effecting a thorough cure is to remove the swelling as soon as it appears. If operation should fail, call in the butcher.

\section{9.-Gloss-Anthrax-Blain.}

This is one of the family of the "anthrax" diseases, so common and so fatal amongst cattle, sheep and pigs. In its nature it is a putrid fever, in which the blood is poisoned with a poison derived from other animals, or self-bred from various causes; and I mention it in this place, instead of classing it with the other maladies of the same character, because it is signalized by the breaking out of a foul and malignant carbuncle in the tongue or mouth.

\section{IN OXEN AND SHEEP.}

Formerly this disease was much more common than it is now, but it is still not unfrequently met with in some parts of Ireland and Scotland. On the continent it is well known as the most rife of all anthrax 
diseases, and as occasionally assuming an epizootic form, and then spreading with great severity and virulence. The course of the disease is very rapid. There are no signs of warning. It begins suddenly, with white pustules on the tongue, lips, roof of the mouth, inside of the cheeks, \&c., which gradually increase in size. There is generally one large pustule on the tongue, where the local mischief is most centered. The pustules then become red and angry-looking, and finally, as gangrene advances, purple and black, and foul with sloughs, or, in bad cases, the tongue falls out in a lump. All this may take place in a few hours, and death in from 18 to 30 from the onset. In addition to these local symptoms, the constitutional disturbance is great, the animal languid and drowsy, and the powers of life profoundly prostrated. Sheep are less liable to this disease than oxen.

\section{IN PIGS.}

This animal is peculiarly subject to several forms of putrid fever, attended with outbreaks of carbuncular swellings, including a malady identical with cattle and sheep gloss-anthrax. The symptoms observed in the pig are trembling and uneasiness, anxious, frightened look, loss of appetite, hot mouth and thirst, with dribbling of mucus from the mouth, and febrile excitement. The appearance of a vesicular or pustular eruption in the mouth is somewhat similar to that found in cattle, as well as the tendency to ulceration, sloughing and gangrene of the tongue and mouth, the great prostration, and the rapid death. Bloody, offensive diarrhoxa frequently comes on towards the last. 


\section{Treatment.}

No time should be lost in treating this disease, and even when treatment is begun early, it may prove fatal from its rapidity and virulence. The diseased animal should be separated from healthy ones, and the same man should not attend upon both. He should be careful not to get inoculated with the matter from the mouth. Nourishing food should be given throughout,-by drench, if necessary.

Tartarus Emeticus is the best medicine to begin with, when the mouth is covered with white pustules.

Mercurius Biniod is the best when the disease is more advanced - the tongue and mouth covered with angry ulcers, from the pustules bursting - when there is reddish and offensive discharge-and swelling of the neck and throat, with difficulty of swallowing.

Arsenicum, when gangrene threatens, and the animal is drowsy and prostrated.

DosEs. - 2 grains of the above medicines every one or two hours ; as the symptoms yield, every four hours. For sheep and pigs 1 grain.

Sulphurous acid lotion* should be frequently applied, both to annul the bad smell, to destroy the contagious property of the discharge, and to avert the tendency to sloughing and gangrene. If there be one large carbuncle, it should be freely opened with the lancet, and then dressed with the lotion-especially if it be true, as some maintain, that the disease is local at first, and only infects the system at large by the virus being absorbed.

* See list of local applications. 


\section{0.-The Mouth and Foot Disease.}

The other names of this disease are-epizootic aphtha - epizootic eczema, the murrain, the epidemic, \&c.

Outbreaks of this disease in different countries are recorded by various writers, during the past two centuries, as having occurred in horses, oxen, sheep, pigs, goats, wild animals, and even in man. It first appeared in Britain, in or about the year 1840, and has since prevailed more or less severely. Some maintain that it was imported there with diseased eattle from infected districts, and that it never spreads except by direct contagion; whilst others ascribe its appearance and fluctuating prevalence to a peculiar condition of the atmosphere, and pertinently ask where the first case came from. The contagionist and the non-contagionist are still engaged in contesting the correctness of each other's opinion. A good deal can be said on both sides, fitting in with facts ; and the question may be regarded as finally settled, when the former can satisfactorily explain the origin of disease in the first instance, and the fleeting visits and final disappearance of the most virulent of human and brute pests.

\section{IN OXEN.}

A period called the period of incubation or breeding, varying from one to four days, intervenes between exposure to the cause and the first appearance of illness. The symptoms of this, the first stage, are as follows: dullness, loss of appetite, shivering, diminution of urine, hot skin, trembling of the muscles of the legs, the snout dry, the head supported on the manger. The mouth is dry and 
hot, red and painful to the touch ; the saliva next becomes stringy and abundant; the teeth are ground; and the breath stinks. If the eruption is going to appear around the hooves and in the interdigital spaces, the animal stamps with impatience and is restless, and brings its legs under its belly, so as to relieve them from the weight of its body; it also rests much, and is loth to rise. In cows, when the eruption is about to appear on the teats or udder, these parts are found to be red, painful and swollen, and milking is painful and not quietly submitted to.

In the second stage, the fever subsides and the eruption appears on the mucous membrane of the mouth, lips, gums, tongue, cheeks, and the muzzle, wings of the nose, as well as on the coronets, and in cows, on the udder. The vesicles vary in size from that of a millet seed to a lentil, are raised in the middle, and are either arranged singly, or run together into a patch ; they contain at first a clear fluid, which afterwards becomes thicker and opaque. When these vesicles burst, the fluid is dis. charged along with the increased flow of saliva, and the surface underneath is seen to be vividly red, and sometimes ulcerated. The animal is constantly licking its nose with its tongue and smacking its lips, and, from the tender state of the mouth, cares little to seize and chew its food. When the eruption appears on the feet, the coronet is swollen and painful, the animal is very lame, the vesicles soon burst, and frequently the entire hoof drops off. The vesicles on the teats are larger and raised, but not pointed in the centre; they are usually surrounded by a red ring, soon burst, and dry into scabs, which peel off gradually. If the cow be milked, the crusts on 
the teats are rubbed off by the milker's hand, troublesome ulcers are apt to follow, the milk is not all removed in consequence of the cow's restlessness, it is mixed with blood, and afterwards with matter, and at last inflammation comes on, which may end in death, or the loss of a portion of the udder.

In the third stage, the disease either abates, or increases. In the former case, the mucous surface and the skin previously occupied by the eruption heals and resumes its natural condition and appearance, the appetite returns, and all the other functions of the body are restored to their healthy state. When the disease gets worse, ulceration takes place, the hoofs drop off, irritative fever continues, the breathing is quick, an offensive discolored discharge dribbles from the mouth, sometimes collections of matter appear on different parts of the body, and death, often preceded by purging, follows from exhaustion.

Such are the chief symptums of a disease, which, sometimes very mild, sometimes very violent, always inflicts severe loss on stock owners from its causing loss of condition and of milk rather than from its fatality.

It has been satisfactorily proved that this disease does not prevent an attack of the lung disease, nor the latter an attack of the former.

There are numerous facts on record both for and against its contagiousness, and all that can now be said is that this point is undecided. Many strong contagionists have been compelled to change their opinion.

Some writers state that not only has aphtha extensively prevailed in man at the same time as in the lower animals, but they have declared that the milk of diseased $3^{*}$ 
cows was the sole channel of communicating the disease. Reynal suggests that those who have had an eruption in the mouth from drinking the milk of an aphthous cow, caught, not genuine aphtha, but an eruptive disease having analagous characters to cow-pox; and this is all the more probable, seeing that cow aphtha is something complicated with what the Germans call false cow-pox (falsche-poken). On this, and all other like questions connected with the alleged transmission of some diseases of brutes.to man, there is much obscurity and no little romance. It appears to be positively proved that the flesh of an aphthous cow does not disease man.

\section{IN SHEEP AND PIGS.}

The symptoms and course of the disease are substantially the same as in the cow, except that the first stage is shorter, and that usually the whole duration is about eight days, instead of varying from eight to sixteen, as in oxen. The hoofs are very frequently cast off, and condition not quickly recovered.

\section{Treatment.}

In the first stage, when the animal is feverish-a condition made known by shivering, loss of appetite, quick pulse, and other symptoms already mentioned, give Aconite.

When the eruption breaks out in the mouth and on the udder, and both parts present the appearance described above, give Mercurius.

Another valuable remedy for the eruptive stage, especially when unhealthy ulceration threatens to come on, 
and when there is purging, shedding of the hoofs, and great weakness, is Arsenicum.

Phosphoric Acid may prove of service in the last stage.

Kali Bichromicum is more especially suitable when the nose is principally involved, and collections of matter appear in different parts of the body. I have also used Carbolic Acid with success in this phase of the disease.

Tartar Emetic I have used in some cases with good effects when Mercurius has been used without much benefit.

When, in addition to the mouth affection, the udder is severely inflamed,-red, tender and swollen, give Belladonna in turn with Mercurius.

DosEs. -20 drops of the above medicines for cattle; 10 for sheep and pigs; each dose every three hours.

Local Applications.-The feet should be kept thoroughly clean by being bathed three times a day with warm water, and after each cleansing, apply the following lotion around the tops of the hoofs and in the inter. digital spaces: Kali Chloricum, 2 drams; Glycerine, 8 fluid ounces; water, 8 ounces,-all to be well rubbed together.

The milk should be regularly drawn off, either with the hand, or by means of teat tubes, and the sores dressed with a lotion composed of Tincture of Calendula, ${ }^{*}$ or a salve made of $1.1 \mathrm{~b}$. butter, 1 oz. Oil Origanum, $\frac{1}{4} \mathrm{oz}$. Tincture of Iodine.

FooD.-During the disease, and afterwards when the

* See list of local applications. 
mouth is tender, the diet should consist of gruel and mashes.

\section{1.-Mumps.}

This disease consists of inflammation of the parotid gland.

\section{IN HORSES.}

In addition to the parotid, the gland on the inner side of the lower jaw-bone may likewise be inflamed. This especially happens in strangles. In ordinary cases, after exposure to cold, or from some other cause, the animal becomes feverish, off his feed, thirsty, \&c.; then symptoms of sore throat come on-cough, difficulty and evident pain in swallowing, and some obstruction to the breathing. The gland, usually on one side, sometimes on both, swells and becomes hard and painful to the touch. The swelling does not go on to the formation of matter, but remains hard for some time.

\section{- IN OXEN.}

Mumps is perhaps seen more frequently in oxen than in horses, especially in winter, during stall feeding.

\section{Treatment.}

Foment with hot water for half an hour, three times a day, and after each fomentation apply a hot bran poultice.

Two medicines are generally sufficient in this disease: Belladonna and Mercurius, six times a day, four hours between each dose.

Dosf.-20 drops in a teaspoonful of water.

When the swelling is so large as to press upon the throat and upper part of the windpipe, and so render 
breathing difficult, it may be necessary to avert the danger of suffocation, by opening the windpipe.

\section{2.-Joint of Lower Jaw Opened. IN HORSES AND OXEN.}

A blow may open the joint between the lower jaw and the temporal bone of the skull. The part is painful and swollen, and a glairy discharge escapes, consisting of joint oil. If care be not taken, the joint inflames, matter runs out, and the bone becomes diseased. Stiffness of the joint may remain, preventing mastication, and thus leading on to starvation.

\section{Treatment.}

Keep the jaws fixed by a head-collar furnished with a strap going round the face and lower jaw above the openings of the nose; feed on fluids until the wound is firmly healed up ; constantly dust on powdered lime whenever the fluid exudes; and give 20 drops of Silicea, three times a day. 


\section{CHAPTER III.}

\section{DISEASES OF THE THROAT.}

\section{1.-Sore Throat-Inflamed Throat.}

\section{IN HORSES.}

Sore throat is the name commonly applied to an inflammatory condition of the back of the mouth, which is more or less affected throughout its entire extent. It is generally associated with, and is a prominent symptom of inflammation of the larynx, although it may exist as an independent disorder. It very often attacks young horses out of condition when brought out of the farmer's hands and put into hot, ill-ventilated stables, and when they are sent to work and placed on unusually good food. This change in their stabling, work, and feeding, predisposes them to be acted upon by exposure to the weather, and sore throat is induced, or some more serious disease of the breathing organs.

In simple sore throat there is some febrile excitement, with loss of appetite, thirst, \&c., followed by hard, dry cough, difficulty of swallowing, quick breathing, and swelling of the throat externally, as well as tenderness when it is handled. The glands under the jaw and below 
the ears are hot, tender and swollen. Subsequently, the cough is looser, a discharge flows from the nose, and the mouth contains a frothy fluid.

In the majority of cases these symptoms gradually subside until health is regained, or symptoms of laryngitis, or of bronchitis come on.

Sometimes the inflammation extends to the guttural pouches, and then, if matter should form, as is occasionally the case, there may be great obstruction to the breathing.

\section{IN OXEN AND SHEEP.}

Sore throat is most common in these animals when they graze on damp lands, during cold springs and autumns. In some seasons sore throat is exceedingly prevalent amongst cattle. The symptoms are much the same as in the horse. We find the same feverishness, difficulty in swallowing, pain and swelling of the throat, and, in addition, there is no chewing of the cud.

\section{Treatment.}

At the beginning of the attack, when the animal is feverish, the pulse and breathing quickened, the membrane of the eye and nose red and injected, the throat tender, and swallowing painful, Aconite will suffice, if given early, to arrest the further course of the disease, in a large number of cases.

Belladonna is best for a later stage, when the inflammation is more firmly established, and is indicated by swelling and tenderness of the glands and throat externally, great difficulty and pain in swallowing, - so much so as to cause fluids to return by the nose; and by a discharge of stringy saliva from the mouth. The exist- 
ence of a dry, irritating cough is an additional indication for its use.

If the last medicine should make no decided impression on the disease, Mercurius should be substituted, or the two medicines may be given in turn, in the same doses, every two or three hours, according to the urgency of the symptoms. This medicine is indicated by somewhat the same symptoms as Belladonna.

Doses. -20 drops for horse and ox ; 10 for sheep and pig ; 4 to 10 for dog. Each dose to be given every two, three, or four hours.

If there be much external swelling and tenderness, foment three times a day with hot water, and afterwards apply a good-sized hot bran poultice to the throat. Give cold water and gruels of linseed and oat-meal, and have the stable well ventilated.

The two following cases are good illustrations of this disease and its treatment:-

\section{CASE I.}

October 26th, 1856.-Symptoms-Pulse 84, and small; respiration 48 , and blowing; nasal membrane vascular; almost constant efforts to swallow, each effort being attended with a clucking sound; frequent hard ringing cough; mouth full of tough saliva, which hangs in strings from the palate when the jaws are separated; submaxillary glands swollen and tender; legs and ears cold; urine scanty and high colored; appetite bad; tongue foul ; fæces lumpy and glazed with mucus.

To have 20 drops of first dilution of Belladonna, and 20 drops of Mercurius, every three hours, alternately.

27 th.-Pulse 72 ; respiration 40 ; glands larger; difficulty in swallowing fluids, part of which returns by the nostrils. Continue medicines.

28th.-Pulse 64; respiration 40 ; glands larger. Hepar Sulphuris 20 drops three times a day. 
29th.-Slight discbarge of a dirty white color from nostrils; glands soft and fluctuating; lanced them, but no matter came out. Continue medicines.

31st.-Pulse 60 ; respiration 28 ; copious discharge from the nose ; some matter from lanced gland; can swallow better; appetite improved. Continue medicines.

November 4th.-Discharge from nose almost ceased; improving generally; lanced other gland, and let out a large quantity of thin, pale yellow matter. Same medicine twice a day.

8th.-Cured.

\section{Case II.}

October 28th, 1858.-Symptoms-Pulse 80 and full ; respiration 48 ; difficulty of swallowing; refuses food on account of the pain attending swallowing; submaxillary glands considerably enlarged and tender; mouth full of tough saliva; very violent cough; loud crowing at every inspiration ; there is evidently a sense of suffocation, as the horse every now and then stamps the ground with his fore-feet, and tosses his head about; anxious expression, \&c. To have Ammon. caust. every three hours.

29th.-Pulse 64 ; respiration 36 ; less wheezing; rough sound in trachea; cough still frequent and inspiration crowing; fluid returns in part by the nose; wouth full of slimy, tenacious mucus. To have Belladonna every three hours.

November 1st.-Yesterday Hepar sulph. was given; copious discharge from both nostrils of a thin matter, mixed with small specks of lymphy substance; the sounds in the throat give the idea of shreds of some substance hanging down from the roof of the throat, and being moved to and fro by the ingress and egress of air. Continue Hepar sulph., 20 drops every tbree hours.

3 d.--Pulse 56 ; respiration 22 ; cough and crowing less ; appetite better; can swallow better, and fluids do not return by nose. Continue medicine.

10th.--Since last report the horse has been going on well, and is now convalescent. 


\section{2.-Putrid, or Malignant Sore Throat. IN HORSES AND OXEN.}

This disease has proved rapidly fatal in both classes of animals. It differs from simple sore throat in the suddenness of its attack and the rapidity of its course, as well as in the greater gravity of the general symptoms. At first, high fever comes on, which is speedily followed by swelling and pain in the salivary glands. The throat, inside and out, swells to such an extent as seriously to interfere with swallowing and breathing; the animal then refuses all food, and the breathing becomes noisy and choking. The breath is most offensive, and gets more so as the disease advances. The animal has an anxious and distressed look, the difficulty of breathing increases, indications of prostration advance, and death soon puts an end to his sufferings.

\section{Treatment.}

Give the medicines advised in the preceding article on "Sore Throat," every hour or two, according to the severity of the attack.

When symptoms of prostration come on, attended with offensive breath, give Arsenicum.

Dose.-20 drops every hour.

In the majority of cases, there is such urgent difficulty of breathing and such imminent danger of suffocation, that the windpipe requires to be opened. 


\section{3.-Throat Diseases in the Pig.}

The pig is subject to two distinct diseases of the throat; one consisting of ordinary inflammation of the mucous membrane lining the throat, palate and larynx; the other, bearing a close analogy to the diphtheria of man, in so far as there is formed a membranous exudation on the diseased surface.

The first form begins with loss of appetite, dullness, disinclination to move, \&c., followed by feverishness, difficult breathing, gasping, cough, bluish color of the mouth, swelling of the tongue, obstructed and difficult swallowing, and choking.

A hard, red, painful swelling appears on the neck, sometimes extending down the course of the windpipe. There is great prostration, and a tendency to gangrene and sloughing.

In the second form, the general symptoms are somewhat similar, and depend upon the local mischief interfering with swallowing and breathing, whilst the constitution at large suffers from the blood being vitiated.

\section{Treatment.}

Refer to the previous articles on throat diseases in other animals.

DosE.-10 drops of the medicines indicated, every one or two hours, according to the violence of the symptoms.

The styes should be well cleaned out and kept clean; the diseased separated from the healthy, and gruels drenched in. 


\section{OHAPTER IV.}

\section{DISEASES OF THE GULLET.}

\section{1.-Polypus.}

Sometimes a polypus is found to hang from the back part of the nose over the opening of the windpipe, giving rise to hackling cough, obstructed breathing, and frequent efforts to swallow. When such a tumor drops into the windpipe, the animal is choked.

Such growths have been observed in both horses and cattle, and often their existence is unsuspected. Whenever the above symptoms are present, the throat should be carefully examined.

\section{Treatment.}

Introduce the hand into the back of the mouth, and twist off the polypus.

\section{2.-Obstruction-Choking.}

IN HORSES.

- The passage of food from the mouth to the stomach may be obstructed by various causes. Horses are sometimes choked by food, such as chaff, bran, carrots, turnips, and such like, accumulating or sticking fast in some 
part of the swallow, or the canal lower down; by the food not being properly chewed and softened by admixture with saliva; and by diseases, such as inflammation, stricture, dilated pouches, in the passage itself. Balls have been known to stick in the throat, or in the lower part of the foodtube, either from being too large, or improperly administered, or from existing stricture.

When the obstruction is in the throat, the animal is in an anxious, distressed state, coughs, slavers, breathes quick, seems to be choking, breaks out in sweats, and frequently retches without being relieved. Water administered, or imbibed voluntarily, returns through the nose. Wherever these symptoms are met with, the hand should be put into the mouth, and the throat explored; if any foreign body be there, it will of course be felt, and should be instantly removed.

When the obstruction is in the neck portion of the food-tube, in addition to the foregoing symptoms, a swelling will be seen in the left side of the neck; and when lower down still, in that part of the tube which lies out of sight in the chest, we find violent retching after swallowing fluids, besides most of the above symptoms, except that the choking is less urgent.

\section{IN OXEN AND SHEEP.}

Here the symptoms of choking are even more urgent than in horses, much more dangerous, and much more likely to end in death. This arises in great measure from the fact that obstruction, from whatever cause, and wherever situated, puts an immediate stop to the process of cudding, and therefore of digestion, so that the food in the stomach undergoes fermentation, gases are generated, 
and symptoms of "hove" come on. To the general symptoms of choking already mentioned as occurring in the horse, there are added in the case of cattle, severe cough and retching, strong muscular contraction of the neck and belly, and violent expulsion of urine and dung.

\section{Treatment.}

As soon as the nature of the case is made out, and there should be no difficulty, because the symptoms speak for themselves, no time should be lost in overcoming the obstruction. In the first place, if it be due to some foreign body in the back of the mouth, or in the throat, the best plan is to open the horse's mouth, pull the tongue well out, and introduce the hand, and so remove the mass or obstructing object. At the same time, the assistant may render efficient service by compressing the throat externally and trying to loosen the obstacle. In the second place, when it lies in the foot-tube beyond the reach of the hand, the swollen part at the side should be rubbed up and down with the flat hand, and such grasping pressure made with the fingers and hands as will be suggested by the intention to loosen the obstacle from where it is fixed, or to break down the mass into smaller pieces. Along with this handling; the animal should drink, or have drenched into it, some tepid water, thin gruel, or oil, in the effort to gulp which, the offending obstacle may be carried down, or in attempts at retching or vomiting, be brought up by the mouth. When this fails, and even at the first, if the obstacle be large and completely immovable, the probang should be introduced at once, the use of which requires some tact, practice, and knowledge of anatomy. Still foiled, there 
is no other resource left save opening the tube, and removing the cause of obstruction. The wound must then be stitched up, and no solid food given for some time.

Usually weakness of digestion remains, for which $N u x$ Vomica, and the other remedies prescribed under "Indigestion," "Hove," may be required.

\section{3.-Dilatation and Stricture.}

This condition of the gullet is sometimes met with in horses. The tube is either dilated near to the stomach, and then we find frequent attempts at retching and vomiting when food accumulates at the part; or it is dilated in the neck-part of the tube, and the swelling occasioned by the lodgment or passage of food is apparent to the eye.

When the dilation is considerable, and the stoppage of food sets up symptoms of choking, refer to the remarks just made on this accident. No medicine avails in this condition.

In Stricture, the canal is more or less diminished in its diameter. Above the point of constriction, the food lodges and in course of time causes dilatation; hence, the two conditions often co-exist in the same animal. Injuries to, and operations on the gullet are apt to be followed by stricture. Fibrous, cancerous, melanotic, and other morbid deposits are at the bottom of most strictures of the gullet.

Very little can be done in the way of treatment, except to give soft foods. Sometimes an operation may be resorted to with temporary benefit. 


\section{CHAPTER V.}

\section{DISEASES OF THE STOMACH.}

\section{1.-Vomiting.}

Vomiting is generally met with as a symptom, rather than as a substantive disease, not only of the stomach, but of other organs also. The same may be said of retching, which is the unsuccessful attempt to vomitto expel the stomach's contents.

\section{IN HORSES.}

Horses rarely vomit, but vomiting is sometimes observed during attacks of colic, ulceration of the mucous membrane of the stomach, distension of the stomach with contraction of the bowel immediately beyond; and more frequently, as a constant symptom, when the foodtube in front of the stomach is contracted or dilated, as to give rise to accumulation of food, as described at page 61, on choking. Vomiting also occurs, when the stomach is burst, an event which sometimes happens in stomach staggers.

\section{Treatment.}

Vomiting will cease on the cure of the disease of which it is a symptom. See following remarks on "Hove," "Indigestion," \&c. 


\section{2.-Hove-Hoven.}

This is the most general name for a very common disorder of cattle, depending on distension of the paunch with gas, given off from the decomposition and fermentation of food.

\section{IN OXEN AND SHEEP.*}

It arises from turning winter-fed cattle into rich clover or grass fields, especially after rain or damp ; from feeding stall-fed cattle on pease, meal, beans, \&c.; and from giving turnips, potatoes, or roots generally. The same hoven condition occurs as a symptom of other diseases, including obstructed gullet, \&c.

The chief symptom comes on suddenly, during, or soon after, eating. A swelling, which gradually increases, rises in the belly towards the left flank, on striking which with the hand, a drumlike sound is heard. The animal stops chewing the cud, appears dull, breathes quickly, and rifts up wind. As the swelling increases, so do the cow's sufferings; the breathing becomes labored and difficult and moaning, the back is arched, the nostrils expanded, the tongue thrust out, the eye-balls anxious and protruded, and saliva dribbles from the mouth. Death is preceded by staggering, falling down, and a greenish discharge from the mouth and nose, and is the result of suffocation. In some cases, the progress of these symptoms is remarkably speedy.

\section{Treatment.}

As soon as distension is observed, stop feeding instantly.

* So-called Hove in the horse, is referred to under "Colic." 
Suddenly dash cold water over the belly-this helps to expel the gas, and to re-excite cudding.

The best medicine, according to my experience, is $A \mathrm{~m}$ monium Causticum, administered as quickly as possible.

DosE.-20 drops of the strong solution in two teaspoonfuls of water, every ten, fifteen, or thirty minutes, according to the urgency of the symptoms and the effect produced. For sheep, 10 drops.

The next most serviceable medicines are Belladonna or Colchicum, one of which should be given if the first should do no good, after a few doses have been administered.

\section{Dose.-The same as for Ammonium.}

In addition to these medicines, mechanical measures must be resorted to from the first, especially if the symptoms appear and advance rapidly, so as to threaten speedy suffocation; these are either to pass a hollow probang into the rumen, or to push a trocar, fitted with a tube, into the left side, midway between the last rib and the haunch bone. In lieu of this instrument, a penknife and large quill or hollow cane, will answer on an emergency. These failing, refer to Crammed Stomach.

In Relapsing Hove, which comes on whenever a cow with weak digestion, or disease of the third stomach, or intestines, eatŝ green food, the treatment consists in regulating the diet, in giving 20 drops of Nux Vomica, three times a day, during the intervals, and treating the attacks when they arise in the above ways.

FOoD, ETC.-In all cases of hove, the greatest attention must be paid, after the attack is over, to the kind of food and the prevention of gorging. Boiled roots are good. 


\section{3.-Indigestion.}

\section{IN THE HORSE.}

Indigestion is derangement of the process by which the food is naturally digested-is disorder rather than structural disease of the stomach, and probably, also, though in a less marked degree, of the liver, intestinal glands, \&c.

It arises from giving indigestible food; allowing too much food after giving too little; eating too much at too long intervals; imperfect chewing, either from diseases or irregularities of the teeth, or from greed; severe work soon after a meal.

The tongue is foul and coated; the mouth slimy; the dung dry and mixed with undigested oats, or hard, glazed and offensive; the urine scanty and thick. The appetite is unnatural or capricious; sometimes the horse eats very greedily, at another he eats very little, or takes one food and leaves others; or he prefers dirty straw to the best oats and hay; or he licks the walls and swallows the plaster from it. He soon gets out of condition, loses flesh, does not thrive, and his skin looks "hide-bound." He sweats easily, and does not work so well as formerly, being weak and spiritless. Very often he has a short, hacking, irritating cough. It is evident from his manner, that he sometimes suffers from smart colicky pains.

\section{Treatment.}

Give Nux Vomica for depraved, fastidious, changeable appetite ; confined bowels ; dung hard, lumpy, and glazed on the surface with mucus; tongue furred and slimy.

Arsenicum is a most valuable remedy when the horse 
is weak and unthrifty, eats little or nothing, conghs frequently after eating or drinking, \&c.

Another good medicine for somewhat similar symptoms to the last is Ferrum, which should be given if Arsenicum does no good.

Dose.-10 grains of Ferrum, thrice daily in a handful of mash; of Nux and Arsenicum, 20 drops in a wine-glassful of water.

FOoD, ETC.-Soft and easily-digested food should alone be allowed, and in small quantities at short periods. The horse should be moderately exercised, and not overworked.

\section{CASE I.*}

On October 5th, 1859, I was called to a horse belonging to $\mathrm{Mr}$. Holland, baker, Manchester. When this horse was bought, three months ago, he was pronounced sound by a veterinary surgeon, although suffering from a cough ; he has been under allopathic treatment ever since, without any benefit; and the V. S. novo states that " that the lungs are rotten."

The symptoms are:-Pulse and breathing unaffected; tongue covered with a dirty yellow fur; mouth contains slims saliva; offensive smell in mouth; bowels constipated; urine, pale brown in color ; coat, dry, staring and devoid of gloss ; abdomen, tucked up; appetite, fastidious; cough, frequent and dry; the horse is in poor condition and does not gain flesh.

Tieaiment.-To have Arsenicum and Nux Vomica 1, 20 drops thrice a day alternately.

14th. "Since last report, the horse has steadily improved; and be is now all right.

\section{IN OXEN.}

In cows, the symptoms do not vary much from those of the horse. The milk is poor in quality, and scanty; purging, belchings and retching are also observed.

*Quoted from "Veterinary Homœopathy Ilustrated," \&c. 


\section{Treatment.}

Thesame as in the horse, both as respects the medicines to be administered, the doses and food.

\section{CASE II.*}

On March 12th, 1860, I visited a cow belonging to Mr. Fletcher, Longsight, near Manchester. Of late this cow has refused her food, and vomited immediately after each meal. Neighboring wiseacres say she has the lung disease, and had better be sold. My opinion was asked on this point.

The symptoms are:-Breathing and pulse unaffected; very little appetite; vomits hay directly it is swallowed; rumination, suspended; dung, scanty; almost total absence of milk; extreme emaciation-reduced to "a bag of bones."

Nux Vomica was given three times a day. In a fortnight the cow was able to eat and digest everything put before her, and she had greatly imprcved in condition. In two months after she was the fattest, the finest and the best milker in the stock.

\section{4.-Stomach Staggers.}

\section{SLEEPY STAGGERS-MAD STAGGERS.}

\section{IN HORSES.}

This name is applied to an assemblage of symptoms, which show that both the stomach and the nervous system are affected. The stomach is more or less crammed with hard and undigested food, as much as 60 pounds weight having been found in it. It is more common in some districts and seasons than in others. Rank grasses, the common rag-weed, rye-grass containing ergot, \&c., have been blamed for its production; but it is also met

\footnotetext{
*Quoted from "Veterinary Homœeopathy Illustrated," \&c.
} 
with after the horse has eaten any other food, especially if he be old, fed on bad food, or be exhausted from illness, over-work, or ill-usage. It often arises when a horse is long fasted and then is allowed to swallow, without slowly chewing, too much oats, bran, hay, \&c. Farm and cart horses are the greatest sufferers.

The bowels are confined, the dung hard and slimy, and little or no urine is passed. Occasionally pains come on in the bowels, and then the animal paws the ground, looks round to his flanks, and lies down and rolls over. Sometimes wind is belched up. Partial sweats break out. The mouth and eyes are tinged yellow. The horse's manner is the most notable feature in the symptoms. He is dull and sleepy, hangs his head on the manger or rests it against the wall ; the eyelids are closed or nearly so; the eyes are partially or wholly insensible to light. From this condition a strong blow or a loud noise may awake him, but presently the same stupor steals over and masters him again. Sometimes the muscles are seized with sudden twitching, and fore-legs drop as if he would fall. The breathing is slow, labored, moaning, or noisy, from throat-rattle. The pulse is full, frequent and oppressed.

These symptoms either abate, leaving the horse more or less affected in his sight and with a weak stomach; or they change their character and indicate excitement of the brain, or MAD STAGGERS, which is a condition occurring either as the advanced stage of stomach staggers, or without the sleepy symptoms above described. As the symptorns of this stage. or form, bear a close analogy to those of inflammation of the brain, the remarks on this latter disease should be referred to. 
Now, the horse shivers all over ; runs his head against the rack, manger, or wall ; stamps wildly as if he were in a passion; plunges about the box; kicks out; rears upwards, and falls backwards on the ground, panting: for breath, and sweating all over; or jumps with his fore-feet into the manger. The eyes are thrust out, fixed and wild looking, and the pupils dilated. The breathing is quickened and the nostrils opened out wide; the pulse hard and accelerated. These attacks recur until one more severe than the rest ends life, often preceded by vomiting, as a sign of ruptured stomach.

\section{IN OXEN.}

Stomach staggers is known in oxen as "grass staggers," and "fardel-bound." Most frequently it is in the omasum, manyplies, or third stomach, that the food is lodged and arrested, although the first and second stomachs are also involved, but to a minor extent. The secretion of the manyplies is absent, and the food is hard and dried into a solid cake. This affection is most common in the spring, when stall-fed cattle are first turned out to grass.

In rinderpest, the third stomach is almost invariably crammed with hard food.

The symptoms do not differ materially from those of the analagous disease in the horse; there is the same stupor in the early stage, and the same wildness in the latter. In addition, cudding is, of course, suspended, the belly is often "hoven," and there may be slight purging. Care as to diet, after the urgent symptoms have been overcome, is even more essential in cows than in the horse. 


\section{Treatment.}

The treatment of stomach staggers consists, in the first instance, in removing the cause, which is an accumulation of food in the stomach, by means of mechanical agents calculated to expel the mechanical obstacle. It is much more difficult in animals than in man, to use the stomach pump with effect, especially when we wish to remove a mass of food often hard, always bulky; indeed, the difficulties are so great as to render this measure practically useless. As much water should be allowed as the animal will drink, no food should be offered, and glysters should be thrown up freely. At least one dose of croton, or aloes, ought to be administered in order to obtain the evacuant action of a powerful cathartic. In addition, Belladonna when there are present the symptoms above described of mad staggers; Opium for those of sleepy staggers; and Nux Vomica, after the urgent symptoms are over, when the stomach is weak and unable to digest food properly.

Dose.-20 drops in a wine-glassful of water, orin a draught of water, every hour or two, according to the violence, of the symptoms. One dose of $N u x$ four times a day, for the subsequent weak digestion.

For some weeks, great care must be taken to give soft food in small quantities frequently.

\section{5.-Crammed Stomach.}

\section{IN OXEN.}

The elegant name for this state is "impaction of the stomach." Of all animals, cows are the most subject to 
it. The rumen, paunch, or first stomach, is more or less stuffed with undigested food, and the beast's life is thereby very seriously endangered. It may arise from eating grains, bran, \&c., and from filling the stomach with grass before there has been time for such a generation of gas as would lead to the production of "Hove." Under some circumstances, a small quantity of herbage will give off a large amount of gas; under others, the paunch is considerably filled with food, and then a comparatively small quantity of gas is evolved. Hence, Crammed Stomach and Hove have many symptoms in common, only they are less quickly produced and less severe in the former than in the latter complaint. The two may be distinguished by crammed stomach wanting the peculiar drumy sound on striking the paunch, by pressure on the swelling on the left side leaving the marks of the clenched fist, and by no gas escaping when the hollow probang is introduced, or the swelling punctured.

\section{Treatment.}

The operation of "Paunching" must be performed without delay; thus: Having properly secured the animal, plunge a sharp carving-knife into the swollen paunch, through the skin at a point midway between the haunch bone and the last rib, and about nine inches from the spine (back bone); and, whilst drawing out the knife, cut a slit six inches downwards. Grasp and hold fast the edges of the wound in the stomach and side of the belly, otherwise the food will drop into the belly amongst the bowels and set up a probably fatal inflammation. Then, lift out the food with the hand. When 
this has been done, sew up the edges of the wound in the paunch with three or four single stitches of fine catgut; and lastly, secure the wound on the cow's side by passing through its lips as many stitches of flexible wire. Only soft food is allowable for some days. The operation is usually successful and speedily recovered from.

Afterwards, treat as for "Indigestion."

\section{6.-Crib-Biting and Wind-Sucking.}

These two tricks, or symptoms of disease, whichever they may be, are confined to the horse; they are mentioned here because they are either the results of a disordered stomach, or frequently lead to it. Certainly, both lower the horse's condition, and would seem to render him more than usually liable to indigestion and attacks of colic. Many horses acquire the habit by imitation; therefore, those addicted to it should be kept apart from others.

A crib-biter fastens his front teeth into the manger, curves his neck and sucks in air with a peculiar noise.

A wind-sucker presses his lips against some hard body, brings his feet together, arches his neck and swallows air, without fixing the teeth. After either of these two performances, the belly becomes enlarged and drumy.

\section{Treatment.}

There are various contrivances in the shape of racks and straps for the prevention of both practices.

If symptoms of indigestion be present, consult the remarks on that subject in this chapter.

In the eye of the law, crib-biting, which has not pro. 
ceeded so far as to induce a disease, or change of structure, or to interfere with the horse's usefulness, is not unsoundness, but a vice; and a purchase, under a warranty that a horse is "sound and free from vice," is void.

\section{7.-Stomach Pain in the Horse.}

I have often observed a class of symptoms, which I believe to arise from pain in the stomach. They are as follows: The general symptoms resemble those of colic ; the horse shows by his manner, restlessness and looks, that he is in pain: he turns his head round to the left side and puts his nose there behind the left elbow joint. Besides, wind (gas) rises up from the stomach, and in its passage along the food-tube causes a waving motion similar to that-only in the opposite direction - which takes place when water or food is swallowed. It is in reality a form, or a symptom of indigestion.

\section{Treatment.}

I have never failed with Antimonium Crudum, and, therefoer, it is needless to refer to any other remedy.

DosE.-20 drops in a wine-glassful of water every half hour, or hour, until the symptoms are relieved.

\section{8.-Loss of Appetite.}

\section{IN ALL ANIMALS.}

This is not a disease in itself, but a symptom of many different discises, both acute and chronic. It is one of the first in diseases attender with febrile excitement. 
It is associated with diseases of the teeth, mouth and stomach; and after recovery from serious illness, some loss of appetite remains.

\section{Treatment.}

When an animal appears to be in tolerable health, but does not eat as he ought to do, give Arsenicum or Nux Vomica, night and morning; or one medicine in the morning and the other at night.

Dose. - 2 grains in a handful of mash, for horses and oxen; 10 drops of the tincture, for sheep and pigs.

Of course an examination should be made to ascertain whether or not actual disease exists. The mouth in particular should be explored for irregular teeth, \&c., especially if the animal "quid" his food. See remarks on this subject, at page 33 . 


\section{CHAPTER VI.}

\section{DISEASES UF THE BOWELS.}

\section{1.-Intestinal Worms.}

\section{IN HORSES.}

The true worms found in the horse's bowels comprise three species of tape-worm; namely, the T'ceniaplicata. T. perfoliata and the T. mamilana; a large, round worm, the ascaris megalocephata; and another, often called a thread-worm, the strongylus armatus.

The first kind are rarely met with, and when they do exist, give rise to little or no disorder. The presence of worms in general is denoted by staring, hind-bound coat; appetite at one time poor, at another greedy ; loss of flesh and condition; occasional attacks of colic, or of diarrhœea, dry cough, \&c. When the worms are found passing from the bowels, all doubt is removed as to the cause of their symptoms.

\section{IN OXEN AND SHEEP.}

In the first animal's bowels, the tape-worms are called Tunia expansa and T. deriticulata ; and the round worms, the strongylus radiatus and the ascaris lumbricoides. 
The Tcenia expansa and several species of strongyli are found in sheep, and produce much the same symptoms as in other animals.

\section{IN PIGS.}

The worms found in the pig's bowels are tcenice, ascarides and a large round worm, with a long name-the echinorhynchus gigas, to young pigs most injurious. This animal is much disturbed by worms-the loins are weak ; the hind legs stiff; the eyes sunken; there are constant grunts, and biting and fighting one with another. In bad cases, the weakness is so great as to induce fatal exhaustion.

\section{Treatment.}

In the treatment of worm cases we must, firstly, destroy and expel the worms ; and, secondly, give such medicines as will improve the mucous membrane of the bowels and its secretions.

For the horse, as a mechanical evacuant, give two croton beans powdered and mixed with a handful of bran mash. Then, for the symptoms above described as the result of worms, give 2 grains of Arsenicum, first trituration, night and morning, in a little mash; or, this failing to do decided good after having been administered for two or three weeks, Ferri Sulphas, in the same way, in 10 grain doses.

For oxen, as an evacuant and as a poison to the tapeworms, Turpentine, half an ounce mixed with olive oil; and as constitutional remedies Arsenicum and Ferri Sulphas, as for the horse.

In sheep and pigs give half these quantities.

The operation of the evacuant medicines should be 
watched, and tape worm, when expelled, either whole or in pieces, should be burned or deeply buried.

\section{2.-The Horse Bot.}

The "bot" is the maggot form of the breeze or gadfly, of which three species-take up their temporary abode in the stomach and entrails of the horse; they are the Estrus Equi, or great spotted horse-fly; the OEstrus Homorrhoidalis, or red tailed horse bot; and the Eistrus Veterinus. The natural history of these creatures is a curious one. Towards the end of summer the fly deposits its eggs on the inside of the horse's knees, on his breast, or on his lips; the eggs are covered with a gluey fluid, which causes them to stick to the hair of these parts. Several hundred eggs may be thus laid on a single horse. They set up considerable irritation and itching, to relieve which the horse licks, or nibbles the skin, and in this manner they are conveyed into the stomach-a transition indispensable to the hatching of the eggs. Here the larvæ at once fix their heads, by means of sharp hooks, into the mucous membrane, where they hang in clusters. During the following winter and spring they undergo no further change, but gradually grow larger. Towards spring, being ready to advance another stage, they loosen themselves, and are discharged from the body along with the fæces. They then find a convenient hiding place, where they change into the form of chrysalis, and sometime afterwards into that of a fly. The second species mentioned above is commonly called the "lip and fundament bot," from two peculiarities-it fixes its eggs about 
the horse's lip, and after quitting the stomach, often adheres a considerable time to the end of the anus.

If a horse out at grass in autumn is observed to be uneasy, going awkwardly, and licking the inside of his legs, or his breast, or, when the lip-fly approaches him, tossing his head and galloping off, there is strong suspicion that he will be troubled with bots next summer. When bots exist in the stomach, there may be no symptoms whatever; but when they are in large numbers, various symptoms of indigestion, attended with loss of condition and flesh, usually arise. All doubt is dispersed when they are seen passed with the excrement, or hanging at the anus.

\section{Treatment.}

Nothing can expel them from the stomach; at the proper time in their development they come away of themselves. The best remedies for the effects produced by them are Arsenicum or Ferri Sulphas.

The first medicine should be given for two or three weeks; and then, after an interval of three days, the second, in case there should not be decided improvement.

In some cases, especially those attended with symptoms of colic and indigestion, I have seen good effects from Nux Vomica.

Doses. - 20 drops of Nux and Arsenicum nigbt and morning; 4 grains of Ferri Sulph.

The following remedy will usually give relief in fifteen minutes: Dissolve one ounce of pulverized aloes and one tablespoonful of ginger in a pint of hot water, then add one ounce of spirits of sweet nitre. Give it all at once. 


\section{3.-Concretions-Dust and Hair Balls.}

\section{IN HORSES.}

A small pebble, or other foreign body, when accidentally swallowed, is often found to be the centre of deposits of earthy matter, leading to the formation of stones, which vary greatly in size. These hard, earthy stones are occasionally found in the horse's stomach, but more frequently in some part of the large intestine. They are met with oftener in some districts than in others-owing probably to the larger amount of calcareous matter in the water drunk.

The dust ball is composed of oats, the dust of oats or barley used as food, and mucous material mixed up and matted together. They often acquire a very large size, and there are often several of them in the same horse. They begin to be formed in the stomach, around any body that will act as a centre-point for deposits, and afterwards pass into the bowels.

\section{IN OXEN, SHEEP, \&c.}

Hair balls are very common in these animals. They consist of hair, thickened mucus and other deposited matters, and begin from swallowing hair when the hide is licked. These usually remain in the stomach, and give rise to no particular symptoms. Besides stomach stones, the dug is liable to have hair balls in the bowels, as well as stony concretions formed around foreign bodies.

The balls often attain an immense size without causing any appreciable derangement of health. Usually, however, they set up obscure symptoms of general ill health 
- the animal gets thin, weak and dull, and has frequent attacks of colic. Towards the last, the bowels become constantly costive, the belly swollen, the back arched upwards, and the breathing quickened. When attacks of pain come on, the eyes have an anxious look, and, in the case of the horse, he sits on his haunches like a dog.

\section{Treatment.}

If it were possible to be sure of the existence of these stones when they are small, it would be comparatively easy and safe to expel them through the bowel by means of a purge; but as this knowledge is wanting, and as the mass when once it has grown large cannot be made to pass along the bowel, nothing can be done but to give relief. A purge is then worse than useless, and is certain to do mischief. During the attacks of pain, treat as for colic.

\section{4.-Costiveness.}

\section{IN HORSES, OXEN, \&C.}

In all animals, costiveness is rather a symptom of many diseases, than a distinct disease of itself; nevertheless, it often assumes the importance of a special disorder in the lower animals, from the circumstance that the nature of their food and the necessity for frequently changing it, induce this condition, which often leads to loss of appetite and pain in the bowels. It is apt to come on from eating old, rough grass-the tough fibers of which resist digestion and softening, and lace together so as to cause accumulation, especially in the rectum. 
Newly-born foals are very liable to costiveness of a dangerous character. The excrement existing in the bowel before the foal's birth cannot be discharged, and symptoms of severe colic come on.

Oxen are likewise occasionally the subjects of simple costiveness, and calves particularly so when first placed on dry food.

In all such cases, we find loss of appetite, uneasiness, indications of belly-ache, straining efforts to relieve the bowels, hardened excrement, \&c.

\section{Treatment.}

Horses should be regularly exercised, and be fed on boiled food, the quantity of oats being reduced for a time. Dogs, also, should be exercised, and have well boiled oatmeal gruel, and occasionally bits of liver. In all animals, the diet should be carefully regulated according to the state of the evacuations. Injections of warm water and soap should not be omitted, especially if the last portion of the bowel be stuffed full. Back-raking the horse is not free from danger. In the smaller animals, it may be necessary to scoop out the accumulation when it lies in the rectum. When colic is present, treat as directed for that complaint.

The best medicines for constipation are Nux Vomica and Sulphur - the former to be given for the first week; and the latter, for the second; and so on in turns as long as may be required. Or, one may be given at night, and the other in the morning.

Doses.--20 drops night and morning, for horses and oxen; 10 drops for foals, calves, sheep and pigs. Many cases do best with 2 grains, night and morning, of Nux Vomica first trituration. 


\section{5.-Colic-Gripes, \&c.}

\section{IN HORSES.}

This, one of the most common and fatal diseases to which the horse is subject, depends on spasm, or cramp of the muscular coat of a portion of the bowel. It arises from a variety of causes-such as dust balls and stony concretions, masses of undigested food, and hardened excrement obstructing the bowel-from over-eating, as when a horse gets loose at night and walks into the corn bin; or naturally has a greedy appetite and gorges himself; or, during the day, has the nose-bag put on whenever he stops, and when brought home at night is again fed freely, or allowed to eat as much hay as he likes -from irregular feeding, as when he is kept short at one part of the day and liberally supplied at another-from exhaustion, following hard work and coupled with improper feeding-from exposure to cold, or drinking cold water when the body is hot-from the presence of worms, \&c. In the great majority of cases, the cause is some impropriety in feeding, and, therefore, if common-sense attention were paid to this point, there would be many fewer cases of colic. When a horse is frequently attacked with colic, there is a strong presumption that he has dust or stone balls in the bowels, especially if the general health and condition breaks down.

Let me sketch the picture of a griped horse. Suddenly he becomes restless, walks about, crouches, paws the ground, kicks his belly with the hind feet, looks often round to his side, \&c. Presently he lies down, and rolls about with more or less violence; sometimes resting on 
his belly and looking round anxiously at his flank, sometimes stretched out full length, sometimes turned on his back, in a state of comparative calm. The pain now remits - he gets up, shakes himself, and begins to eat or nibble. Before long, another attack, more severe than the first, seizes him, and the old symptoms are repeated with greater violence than before. He throws himself about wildly, utterly indifferent to the injuries he selfinflicts; his eyes stare and look anxious; he breathes fast; his skin is more or less covered with sweat; he tries to stool but passes little or nothing; he perhaps voids a few small, hard lumps of dung. After several such displays, the attacks become milder and fewer, and finally cease ; or they increase in number, and then be exhibits indications of exhaustion; walks unsteadily, or reeis round the box; finds no temporary relief save when lying against the wall on his back; his muscles twitch ; his breathing is quick and groaning; his pulse small and hurried; he breaks out in patches of cold sweat; the retracted lips expose the clenched teeth; and ere long, death closes the painful scene.

Colic may continue from half an hour to a day, and may lead to death from rupture of the bowel, or of the midriff, or from twisting of the bowel.

There is another form of this disease, called by some Flatulent Colic, or Acute Indigestion, which arises from eating too fast, over-loading the stomach, drinking too much water, working on a full stomach, eating turnips, carrots, potatoes, rank grass, \&c. The food either remains undigested, or it ferments and gives off' gas which distends the stomach and bowels. It corresponds to the "Hove" of oxen and sheep. The symptoms are similar 
to those first depicted; with the addition, that the belly is more or less bloated, and drumy when struck,- - that rumbling noises are heard within, and wind is discharged from the bowels, - and that the horse frequently retches, and may succeed in vomiting. This is the more dangerous of the two forms, and very frequently terminates in rupture of the stomach.

\section{IN OTHER ANIMALS.}

Oxen are not so often the subjects of this disease as horses and dogs; when it does attack them, the causes and symptoms are the same. Dogs, during the severity of the attack, arch up their båck, draw their legs under the belly, and pull the tail between their hind legs.

\section{Treatment.}

Aconite is indicated by the following symptoms: when the attack has been induced by a chill, or by drinking cold water when the body was hot; when the animal's behavior, as above described, shows that he is in great suffering; when he frequently tries to pass urine and dung; when the belly is tender, swollen, and wind rumbles in it.

Ammonium Causticum is, according to my experience, the only single medicine which can speedily cure the largest proportion of colic cases. It is more especially suitable for "windy colic."

Nux Vomica is the best remedy when the attack arises from eating indigestible food, or from over-eating, or from accumulation of excrement; when hard, dry lumps are discharged; when the horse makes straining efforts to urinate and dung, without any result, or with 
but little; when the pain is not of the most violent character, and the horse does not knock himself about savagely, but lies a good deal on his side, restless and uneasy, and every now and then looks round to his side.

Colocynthus is indicated in cases attended with most severe pain, causing the animal to roll about violently; also when the attack appears to result from eating green food, and the belly is much distended with gas; and when wind and watery motions are discharged by the bowel.

Doses.-20 drops of any of the above medicines for horses and oxen : 10 for sheep and pigs. Repeat each dose every fifteen or thirty minutes, according to the violence of the symptoms.

When the above remedies fail, I use a medicine composed of $1 \mathrm{oz}$. aloes, $1 \mathrm{oz}$. sweet nitre, 1 tablespoonful of ginger. Dissolve the aloes and ginger in 1 pint of warm water, then add the nitre. When cool, give to the patient. This will generally cure in fifteen minutes If it fails, give another dose in thirty minutes. This remedy is also a sure cure for bots.

I have given chloroform for colic, 1 teaspoonful in twice the quantity of water, with good effect.

There are several other medicines which are of use in exceptional cases, but the above are sufficient to cure the great majority, and that more speedily than the ordinary plan of giving turpentine, purges, \&c. Injections of warm water should be thrown up occasionally. The operation of "back-raking" relieves the rectum, but injections are safer and just as effectual. I have known stable-men and grooms thrust their hand through the bowel, and, of course, thus destroy the horse. The ani- 
mal should be turned into a loose box, with plenty of straw to roll on. Compelling a griped horse to walk and trot is downright cruelty, and a most dangerous practice. After the attack is over, give soft food, and exercise gently for two or three days.

\section{CASE.*}

On October 24 th, 1855 , I was sent for in great haste, to see a horse belonging to Messrs. Syddall Brothers, Chadkirk, near Manchester. During the last twefve months this horse has had several attacks of colic, which have always yielded to Aconite. 1t has given only slight relief in the present attack, which has lasted five hours. I found the following symptoms: the horse is lying stretched out full length: when made to rise, he is no sooner up than he draws himself together and drops down again; he turns his head and frequently looks at his abdumen; he has voided a small quantity of dung, covered with mucus; the eyes roll about, and the eyebrows quiver, \&c,

To have Nux Vomica. There was no return or the pain after the first dose. Twenty minutes after the first another dose was given. In an hour from the first dose the horse was standing up, eating hay, and well.

\section{6.-Diarrhœa.}

\section{IN HORSES.}

The frequent discharge of liquid excrement, uncolored by blood, which constitutes diarrhœea, is a very frequent symptom of disease. "Washy" animalsthose with narrow loins and great width between the ribs and haunch bones-are peculiarly subject to it; some horses without this make are constitutionally predisposed to it; hunters, excited with going to hounds,

* Quoted from "Veterinary Homœopathy Hustrated." 
are often troubled with it. Change of diet, bad or improper food, often induce it, especially in association with over-work. Super-purgation is the result of giving purges in too large doses, or too frequently, - a practice much less common nowadays than in past years, and one that has killed many a horse. Purging also arises towards the end of influenza, and other diseases attended with prostration, and is a constant symptom of disordered liver.

The evacuations are passed frequently, with straining, and discharge of wind. There are often indications of belly-ache; such as uneasiness, looking round to the flank, pawing the ground, rolling over, \&c. When digestion is imperfect, the oats are passed undigested. The discharges are offensive, and mixed with more or less of slime. In bad cases, diarrhœea is a dangerous disorder in the horse, and may destroy life. This event may be apprehended when the legs are cold; the surface covered with cold sweats; the breathing quickened; the pulse small and weak; the appetite gone; the strength rapidly reduced; and the flesh wasted away.

In some cases, diarrhoea is of the lingering (chronic) form.

The following case illustrates the symptoms and treatment of a severe attack of diarrhœa, excited by an overdose of aloes, in a horse previously weakened by work and bleeding :-

\section{CASE.*}

In July, 1853, a cart horse beionging to Mr. 'Tait, bleacher, Heaton Mersy, near Manchester, was unwell, and the farrier engaged on the premises thought the best plan to resture the

*Quoted from " Veterinary Homæopathy Illustrated." 
animal to health would be to abstract six quarts of blood and give a dose of aloes.

I found the following results: Pulse 84, and so feeble and threadlike that it is with difficulty it can be felt at all; breathing 40 per minute, and laborious ; glassy staring eyes; legs, face and nose cold as death ; general appearance haggard in the extreme; offensive smell from mouth; appetite quite gone, in fact has neither eaten nor drank within the last four days; extreme debility, so that when made to move, he staggers as if he were about to fall; during four days the purgation has been excessive, and now a dirty, water-like fluid is streaming down his hind legs.

To have Aconitum and Arsenicum of the first dilution, in 20 drop doses, every three hours alternately.

On the fifth day from my first visit, the horse's pulse and breathing were normal, his appetite had returned, and, in short, his improvement was so great, that he did a little work. On the following day he resumed his ordinary task of carting bleached goods between his owner's works and Manchester.

\section{IN OXEN AND SHEEP.}

In oxen, diarrhœa is a frequent symptom of the lung disease and rinderpest. It is very common when the diet is changed, and when cattle are turned into new grass pastures. Sucking calves often have the "white scour," from the milk they drink resisting digestion, and passing out curdled; it then acts as an irritant on the bowels. Lambs are likewise subject to this form of purging when they are suckling, and to another form arising from eating too rich grass in spring.

\section{Case.*}

Mr. Johnson, farmer, Moston, near Manchester, called upon me on September 16th, 1857, respecting a cow. The only particulars of her history that could be learnt were these: The animal began

*Quoted from "Veterinary Homœopathy Illustrated." 
to be purged six weeks ago, and although numerous compounds, or "cow drinks" had been given, the ejections continued as copiously as ever. She had, consequently, been reduced to a heap of bones; the secretion of milk was suspended, and the appetite gone.

I gave her Comphor and Veratrum, each in 20 drop doses, to be given every three hours alternately. The owner reported, after these medicines were all administered, that "the cow is quite well, and is coming to her milk."

\section{IN PIGS.}

In this animal, the causes of diarrhœa, and the symptoms attending it, present no great variation from the same in other animals.

\section{Treatment.}

Bryonia should be given when the purging arises from drinking cold water or being exposed to sudden changes of temperature, whereby perspiration is checked, and chill is received; when the evacuations are very fluid and passed almost involuntarily; when they contain undigested food, curdled milk, \&c.; when it seems likely that drinking impure water, containing vegetable matter, as on moors or marshes, has excited the attack; and when eating and drinking bring on purging.

Arsenicum is indicated when the diarrhoea is attended by violent pain in the bowels, as may be inferred from the animal's conduct; when the discharge is watery, slimy, greenish, or brownish; and when the animal becomes depressed, weak, thin, and does not eat.

Mercurius is suitable for cases attended with straining efforts, and protrusion of the bowel at the anus; when the motions are slimy, frothy, dark, and offensive, and mixed with bile and perhaps with streaks of blood; when there are retching and vomiting, \&c. 
China is a valuable remedy for the chronic form of diarrhœa, especially if the discharge is not accompanied by pain; when there is loss of appetite, failure of strength, and wasting.

Nux Vomica is indicated when the diarrhœea is attended with symptoms of indigestion, as mentioned at page 67 ; and when purging alternates with constipation.

Colocynthis is required in those cases of violent colic which are sometimes attended with looseness.

Sulphur is of service when the purging is attended with eruptions-as is often the case in dogs, for example ; and when milk disagrees and is the cause of the looseness, as in sucking calves and lambs.

Doses.-20 drops for the horse and cow ; 10 drops for sheep and pigs; from 4 to 10 for calves and lambs; to be given in a teaspoonful of water for the smaller, and a wine-glassful for the larger animals. Each dose is to be repeated every one, two, or three hours according to the violence of the symptoms, increasing the interval between each as improvement sets in. For chronic cases, two or three times a day.

Food.-If the food be faulty, withdraw it. Starch gruel, and wheaten flour gruel are good drinks; and for dogs, ground rice mixed with a little beef tea. In cattle, change of pasture is advisable. To suckling animals, or young animals requiring milk, give from a teaspoonful to a table-spoonful of Rennet.

\section{7.- "Braxy" in Sheep.}

The fatal disease known amongst shepherds by this name is met with in different forms; or the symptoms vary so much as to constitute different forms; and probably totally different diseases, are confounded together 
under one general designation. Some cases are associated with disease of the stomach and bowels-" bowel sickness." For further particulars, refer to the chapter on Blood Diseases.

\section{8.-Dysentery-Bloody Flux.}

Dysentery consists in inflammation of the mucous membrane of the bowel, more especially of the large intestine and rectum, is attended with discharge of blood and lymphy shreds, and is prone to end in ulceration. Some cases of diarrhoea run on to, or become mixed in symptoms with those of dysentery.

\section{IN HORSES.}

It is less frequently observed in horses than in other animals. Bad food, exposure and overwork strongly predispose to it; and grazing on damp, marshy pastures excites it. Young, well-conditioned horses have the acute form; and old, done-up horses the chronic.

The usual symptoms are as follows: Pulse small and quick; breathing quickened; great thirst; no appetite; frequent efforts to relieve the bowel, often without success, attended with symptoms indicative of pain in the belly; the straining is so severe sometimes as to force out the end of the rectum a short distance; the discharge consists of excrement coated over with congealed blood, of slimy mucus mixed with dark blood, and of lymphin the latter case presenting a fatty appearance, and hence the old name given by the farriers, "molten grease," from a notion that the fat of the body was melted down and then ejected by the bowels. If the disease continues 
unchecked, the horse becomes exhausted, very weak, and greatly wasted.

\section{IN OXEN.}

Dysentery complicates rinderpest; sometimes occurs as an epizootic; and usually attacks, in this country, cows confined in dirty, ill-ventilated shippons. It also arises from drinking impure, or stagnant water; from eating rank grass, or pasturing on low marsh lands; and when over-driven cattle are fed on bad food, and are exposed to severe weather.

Shaking, dullness, anxiety, dry skin, slightly rough hair, and uneasiness of manner. In some cases the bowels are bound, the dung hard, dry, in small lumps, and pain attends their discharge; in other cases, the dung is soft and discharged frequently, and the animal wastes and loses her appetite and spirits. In other instances, again, the purging is almost constant and severe from the first, attended with pain, straining efforts, and thrusting out of the fundament. In this stage the discharge is watery, mixed with white shreds or darkcolored blood, with little or no dung, and it has a horribly bad smell. These violent symptoms may disappear, but the purging and wasting continue as bad as ever, or they stop for a short time, then begin again, and continue until the animal is worn out by the constant draining; or they are arrested, and improvement begins and goes on slowly. Medicine will do no good in those very severe cases in which the wasting is so excessive that the animal is reduced to a living heap of bones, the joints swelled and covered with sores, the failure of strength great, the eyes hollow and dim, the teeth loose, and the parts under the jaw enlarged; the body covered with 
vermin; the discharge mixed with blood or matter, and having a horrible smell, and the body bedewed with cold sweat.

\section{IN SHEEP AND PIGS.}

In these animals dysentery arises from bad food, overcrowded, ill-ventilated styes, \&c. ; it is signalized at the commencement by dullness, feverishness, thirst, loss of appetite, \&c, and at a later period, by pain in the belly, severe straining, slimy, bloody discharges, followed by rapid wasting, and failure of strength.

\section{Treatment.}

Aconite is frequently useful at the outset of the attack, when the pulse is quickened, the mouth hot, \&c. ; and when there are indications of pain in the belly.

The most valuable remedy, however, is Mercurius Cor . rosivus, which is specially adapted for those cases which are characterized by severe straining; protrusion of the end of the bowel ; discharge of pure blood, or of slimy mucus mixed with blood, and of hardened excrement; frequent urgings to relieve the bowel; symptoms of belly-ache before, during, and after each action.

Colocynthis comes next in value to the last medicine ; it is more particularly indicated when the belly is distended and the seat of severe colicky pains; when the motions are slimy and streaked or mixed with blood; and when the animal is restless and occasionally shivers.

Ipecacuanha may sometimes be serviceable when the evacuations consist chiefly of slimy inueus, with or without admixture with flakes of lymphy matter.

Acidum Phosphoricum should be tried after Mercurius and Culocynthis, if the discharge of blood continues ; and 
Arsenicum when the discharge is offensive, and passed almost involuntarily, and when there are great weakness, wasting, and dullness.

Dosfs.--20 drops of any of the above medicines for horses and oxen; 10 for sheep and pigs. Repeat each dose every one, two or three hours, according to the urgency of the symptoms.

Injections of starch gruel are generally of great value in soothing the bowel; they may be thrown up two or three times a day. The food should mainly consist of gruel; and after recovery, of soft fodder, \&c. For some time, the diet must be very carefully regulated.

\section{9.-Enteritis-Inflammation of the Bowel. IN HORSES.}

- In this disease, the mucous and muscular coats of the bowel are inflamed, at first and chiefly. It arises from causes similar to those which have been enumerated as giving rise to colic, and also from twists and displacements of a portion of the bowel.

In the majority of cases, enteritis begins slowly with dullness, staring coat, restlessness, loss of appetite, quickened pulse, \&c.; sometimes shivering is the first symptom; at other times, colic. The pain, judging from the animal's behavior, varies in severity. The belly very tender under pressure, and tucked up. Constipation is usually present, unless when the attack is caused by irritant poisoning; then there is diarrhoea, attended with straining. The mucous membranes of the eye and nose are red and highly injected. At a later period of the disease, symptoms of prostration come on. The pulse 
becomes small and weak, and can hardly be felt or counted; the breathing quick, jerky, and sighing; the skin covered with cold, clammy sweat; the legs and ears as cold as ice; the strength rapidly declines; the muscles all over the body tremble and twitch; and lastly, convulsions come on, and life is soon extinct.

\section{IN OXEN, SHEEP, \&C.}

In oxen, enteritis is met with, either as an occasional casual disease, or, from obscure causes, prevailing more or less widely amongst the cattle of one locality. It begins, as in the horse, with dullness, loss of appetite, \&c., followed by hot, dry mouth; dry muzzle; tenderness of the belly, which is bloated with gas; quick pulse and breathing; bowels constipated, or profusely relaxed. The animal is evidently in pain; it moans, grinds its teeth, lies down and gets up, and wanders about in a state of half unconsciousness. Before long, its strength fails; it staggers, falls down, cannot get up, plunges about, and dies.

In sheep, the symptoms are essentially the same.

In pigs, we observe the same dullness and uneasiness, accompanied by grunting and squealing, and usually by constant retching, or vomiting.

\section{Treatment.}

Aconite is generally the best remedy to begin with, especially when the animal is feverish and in great suffering.

Belladonna is indicated by fullness of the belly; tenderness on pressure; redness of the eyes and nose; and symptoms of severe pain.

Mercurius is indicated by great thirst; tender belly ; 
watery, offensive evacuations with more or less urging and straining; the evacuations also slimy and mixed with blood; prostration of strength; shivering; and perspirations.

Arsenicum is a valuable remedy for anxiety, restlessness, and rapid loss of strength; for small weak pulse; for retching and vomiting; for looseness of the bowels ; for great chilliness of the ears and legs.

E Doses.-20 drops for horses and oxen; 10 for sheep and pigs.

Repeat each dose every fifteen or twenty minutes, according to the severity of the symptoms.

The remarks on "Colic " and "Peritonitis" should be consulted.

When enteritis is the consequence of poisoning with irritants, the treatment consists in administering a chemical antidote suitable to the special poison, and in giving linseed gruel freely. For the symptoms which remain, treat as directed above; or as laid down under "Diarrhøea," "Constipation," "Indigestion," \&c., according to the indications presented by the animal's condition.

Apply fomentations externally, throw up injections of starch, and give gruel freely.

\section{0.-Enteritis with Exudation. IN HORSES AND OXEN.}

This is a form of enteritis, not unfrequent in these animals, especially the latter, signalized by the discharge of false membrane. "The "molten grease" of the farriers was probably the disease under consideration. The shreds, and ribbons-often many feet long-of false membrane, are frequently mistaken for worms. In other 
respects, the symptoms do not differ materially from those of enteritis as described above; excepting that there is less prostration and fewer deaths-in fact, recovery gen. erally takes place as soon as the membrane is expelled. With respect to treatment, consult the remarks on "Enteritis " and "Dysentery."

\section{1.-Peritonitis.}

\section{IN HORSES.}

Peritonitis is inflammation of the serous membrane which lines the cavity of the abdomen and covers the contained organs. The inflammation may begin at a certain spot, and rapidly spread throughout the whole extent of the membrane, being confined exclusively to the peritoneum from first to last. Those portions of the peritoneum which cover the liver, the stomach, the intestines, \&c., are more liable to take on inflammatory action than that portion of it which lines the wall of the abdomen. The peritonitis is then partial, and is limited to the particular organ which the membrane covers; and it is the secondary result of inflammation beginning in the proper tissue of that particular organ.

It is caused by exposure to cold and damp, by food or excrement passing through the ruptured stomach or bowels and falling into the cavity of the belly, and especially by stabs, and the wound of castration.

The acute form begins with shivering, \&c., followed by loss of appetite; great thirst ; constipation ; seanty urine ; hard, wiry, quick pulse; labored breathing; dilated nostrils; anxious expression; and indications of pain. The 
animal looks round to his flank, paws the ground, crouches, \&c., as in colic. The belly is extremely tender to the touch, hot, and tense. Increased quickness and feebleness of pulse, clammy sweats, short breathing, prostration, and unconsciousness usher in death. If the disease has resulted from the operation of gelding, a few days afterwards, the yard and sheath are painful and swollen, and little or no matter flows from the wound. The animal, if in a loose box, rests his hind-quarters against the manger wall. The breast and legs also swell. Then he lies down, looks anxiously to his side, and a few hours afterwards expires.

In the chronic form, the symptoms are more obscure. The animal is dull, off his feed, uneasy, paws the ground, looks round to his side, walks awkwardly with his hind legs, breathes fast, passes little urine, gets thin and weak, and looks unthrifty in his coat. The belly is tender to the touch, it gradually increases in size, and when percussed yields a feeling of fluctuation, from the presence of fluid. There is now Dropsy of the BeLly.

\section{IN OTHER ANIMALS.}

Oxen and sheep, as compared with the horse, bear injuries of the belly almost with impuuity, and the same remark also applies to other animals. This has been tested by injecting lactic acid into the dog's belly, and by the operation of "spaying" swine, without serious results ensuing, as a rule. When it does occur, either as the consequence of wounds, or injuries, or from other causes, the general symptoms are similar to those found in the horse, as above detailed. 


\section{Treatment.}

Aconite is indicated at the commencement of the attack, for shivering, scanty urine, restlessness, followed by quick pulse and breathing, and general febrile excitement.

Belladonna is required for tenderness and distension of the belly; quick, short, distressed breathing; and for symptoms of belly-ache.

Bryonia is suitable when there are confined bowels; scanty urine; anxious, hurried breathing; and swelling of the sheath, breast, \&c.

Mercurius is indicated for tenderness of the belly to outward pressure; retching, vomiting, \&c.

Arsenicum is indicated for all the symptoms which are met with in the last stage; such as weak and small pulse; great weakness; and also when the pain suddenly subsicles, cold sweats break out, and the pulse becomes almost imperceptible-symptoms which are almost invariably the precursors of death. Swelling of the sheath and breast, and scanty urine, are additional symptoms.

Doses.-20 drops for horse and cow; 10 for sheep and pigs. Repeat each dose every two or three hours, according to the violence of the symptoms.

When peritonitis arises from rupture of the stomach or bowel, it cannot but end fatally. Those cases that follow castration, very often terminate in death within five or six days after the operation. When an abscess forms in the cord, it will be necessary to open it, and to remove a portion of the cord.

Fomentation of water as hot as can be borne, should be assiduously applied to the abdomen throughout the continuance of the disease. In some cases it may be desirable to use injections. 
When fluid accumulates in the belly; the remarks on ascites should be consulted.

\section{2.-Wood Evil-Moor Ill. IN OXEN, \&C.}

The disease thus named is, rightly or wrongly, described as a dysentery due to local causes. It is found amongst cattle which graze on woodland pastures, moors, peaty and ill-drained lands; and is believed to be caused by eating plants and shoots containing much astringent matter. It is most common in spring and autumn.

This disease begins with diminution of all the secretions and excretions- the bowels are costive, the urine scanty, the milk lessened, \&c. Cudding is suspended. Before long, the animal becomes dull, refuses food, drinks eagerly, has a hot and dry mouth, and the pulse and breathing are increased in frequency. The mucous membrane of the eye and nose is injected and yellowish. The urine becomes dark from admixture with blood, and smells strong of ammonia. The fæces are hard and coated with mucus alone, or tinged with blood; and become subsequently liquid, offensive, and still more bloody. At the same time, the animal manifests all the ordinary symptoms of colic. If the disease should remain unchecked, the purging increases, the belly becomes distended with gas, rapid wasting follows, and death ensues.

\section{Treatment.}

The first step is at once to remove the affected cattle to a new pasture. The same treatment should be adopted for this disease as has been recommended for "dysentery." 


\section{3.-Twists, etc., of the Bowel.}

Various deviations from the natural position of the bowel are discovered in most of the lower animals. Sometimes the bowel is twisted upon itself, or entangled by fibrous bands, or strangulated by tumors with a long stalk winding round the intestine. One of the most common forms is called Intussusception, where a portion of the bowel is drawn within the canal in the same man. ner as the finger of a glove may be drawn inwards. The result is the same in all-the bowel is obstructed, no fæces can pass along it, symptoms of colic appear, and the animal dies of inflammation. Cattle and sheep are more subject to this last deviation than the horse.

There are no symptoms during life which indicate with certainty the existence of these serious conditions. The animal exhibits the usual indications of suffering violent pain in the belly, such as are described under "colic." In the great majority of cases, horses sit on their haunches like dogs, and this is held by some to be characteristic.

All such accidents are beyond the reach of medicine. It has been suggested that the belly should be opened and the entanglement unravelled. The suggestion is practically useless, because there are no certain distinctive marks between simple colic and twists. Attempting to cure colic by opening the abdomen would scarcely be good surgery.

\section{4.-Hæmorrhoids-Piles.}

\section{IN HORSES, OXEN, \&c.}

Piles are small tumors, consisting of enlarged veins at the end of the rectum. They occur less frequently in 
the horse than in oxen and sheep. In the latter animals, we observe stiffness in the hind legs, and disinclination to move. The freces are tinged with blood. The secretion of milk is diminished and cudding arrested. An examination makes known the existence of tumors in the bowel. When these tumors burst, congealed blood is discharged. In most cases there are symptoms of feverishness, such as quick pulse, hot mouth, loss of appetite, dry snout, \&c.

\section{Treatment.}

Aconite is indicated when the animal is off his feed, restless, and feverish; when the part is hot and tender to the touch; and when inflammation arises in the piles, especially if it extend, as is occasionally the case, to a large part of the rectum.

Nux Vomica and Sulphur, either singly, or in alternation, are especially indicated when the piles are dependent on constipation.

Mercurius is required when, besides costiveness, there is a discharge of slimy mucus, alone or mixed with blood.

Hepar Sulphuris is of great service when matter is forming.

Doses. -20 drops of any of the above remedies for horses and oxen, three or four times a day; 10 for sheep and pigs.

The part should be frequently bathed, and kept thoroughly clean, and nothing but soft food should be allowed till the complaint is cured.

\section{5.-Ascites-Dropsy of the Belly.}

Dropsy of the belly results from inflammation of the peritoneum, and from diseases of the liver, spleen, \&c. 
The belly is distended, and it fluctuates when percussed. The legs and sheath are swollen, and pit on pressure. The urine is scanty and the bowels constipated.

\section{Treatment.}

Ascites unconnected with organic disease, is generally curable; but when dependent on diseases of the liver, spleen, heart, \&c., it generally ends fatally.

The medicines most likely to be of service are the following :-

Aconitum, when the ascites is of the active kind, with some febrile disturbance and tenderness of the abdomen.

Mercurius, when the ascites is caused by disease of the liver. It is also indicated in active dropsies with febrile symptoms.

Arsenicum, when the ascites follows asthma, or the disappearance of skin disease, or is caused by liver disorders; when the urine is scanty, the debility and wasting considerable, the countenance pinched and anxious, and the legs swollen.

Bryonia is particularly indicated in those acute cases which are brought on by sudden changes of temperature, or by exposure to cold when the body is heated.

Helleborus, Digitalis, Squilla, and Cantharis, are also of service to some cases.

D oses. - 20 drops for horse and cow ; 10 for sheep and pigs; every four hours.

\section{6.-Protrusion of the Rectum.}

\section{IN ALL ANIMALS.}

The disease which bears this name is not protrusion of the rectum, but eversion of the mucous mombrane of the 
rectum. During the act of stool, it is natural for this membrane to be a little exposed, and when the act is over, to be again removed from sight. The same thing occurs to a thickened mucous membrane, perhaps also the seat of piles; with this difference, however, that the eversion is greater and is not temporary. Under the irritation of piles, worms, drastic purges, attacks of diarrhœa, or of dysentery, \&c., the action of the bowel is increased and there is violent straining. The coats of the bowel and the muscular sphincter become lax, and folds of mucous membrane appear externally. But in true prolapsus, there is protrusion, not of one coat only, but of all the coats of the intestine-a comparatively rare accident.

The symptoms are obvious enough.

\section{Treatment.}

In treating a case of this kind, the particular cause must be discovered and removed; for until that be done, the eversion or protrusion will return shortly after the part has been put into its right place. The part should be thoroughly cleansed with douches of cold water, for the purpose of removing dirt, and, by astringing its vessels and reducing its size, enabling the operator to return it with greater facility. After having dried the surface, the part should be returned as gently as possible. The difficulty is, not to get it in, but to keep it in. Cold injections are beneficial. The remedies for piles, or for worms, or for diarrhoa-whatever be the cause of the disorder - must be given. In obstinate cases, which do not yield to these measures because of great laxity of tissue, an operation is necessary. 


\section{CHAPTER VII.}

\section{DISEASES OF THE LIVER, SPLEEN, ETC.}

\section{1.-Jaundice-The Yellows.}

IN HORSES, OXEN, \&C.

Jaundice occurs in horses as a symptom of inflammation of the liver, and frequently of inflammation of the lungs; in oxen, as a symptom of rinderpest, and in all animals as a symptom of congested and of sluggish liver, as well as of obstruction or pressure acting on the gallducts. It is met with in sheep more frequently than in other domestic animals.

The symptoms of simple jaundice are sufficiently obvious. The mucous membrane of the eye and the skin are tinged with a more or less deep yellow color. The urine is dark colored, from the presence of bile in it, as may be known by the yellow stain produced on linen moistened with urine. The fieces are hard, dry, and covered with slimy mucus. The tongue is furred and slimy, and the breath offensive. There is little or no appetite. Wasting of the body and swelling of the legs ensue. 


\section{Treatment.}

When there does not appear to be any other disorder present save jaundice, the following remedies usually prove the most serviceable :-

Aconite, at the beginning of such cases as are attended with feverishness-hot skin, dry mouth, thirst, quickened pulse, \&c.

Mercurius is indicated when there is pain in the right shoulder, as is shown by lameness of the right leg and tenderness on pressure; by the yellow color of the eyes and skin; by the slimy evacuations; by loss of appetite, \&c.

Chelidonium Majus is useful for distension from wind, dark urine, costive bowels, coated tongue, yellow eyes, whitish fæces, \&c.

Doses. -20 drops for horses and oxen; 10 for sheep and pigs. One dose every three hours.

The treatment of jaundice as a symptom is of course that of the major disease, whatever it may be. Also refer to the following remarks on inflammation of the liver.

\section{2.-Hepatitis - Inflammation of the Liver. IN HORSES.}

Inflammation is often mistaken during life for congestion-in fact the two conditions can scarcely be distinguished in the domestic animals. Not only so, but the symptoms of either one or the other are so obscure and ambiguous as often to be misconstrued or overlooked. Both arise chiefly from high feeding, and hence are often found in city dray-horses.

In the acute form of the disease, the horse is dull, 
listless, and unwilling to move; the fæces are dark and lumpy, sometimes of a lighter color than usual and a most offensive smell; the urine high-colored and scanty ; the appetite bad. The feverishness may be very slight, or it may run high. The membrane of the eye is yellow; the mouth soapy and foul. Sometimes the pulse is quickened, sometimes remarkably slow. Lameness of the right leg is common, from pain in the shoulder, just as in the human disease ; and this lameness is often attributed to a wrong cause, and mis-treated accordingly. In bad cases, the pulse becomes weaker, the breathing quickened, the legs cold, and the animal, weak, sleepy and staggering, dies ere long. In some cases, dropsy comes on.

In the chronic form, the symptoms are of much the same character, only they are slow in their progress. There is pain in the right shoulder, known by flinching on pressure, lameness, and pawing the ground.

Inflammation of the liver ends in recovery or in abscess; or in the chronic form which lays the foundation of incurable enlargement, and softening. In oldstanding disorganization of the liver, death often takes place from rupture and internal bleeding. If a fat horse with a low pulse and lame in the right fore-leg is suddenly seized, after severe exertion or even without, with colicky pains, anxious expression, cold legs and ears, and great prostration, it is probable that the liver has been ruptured. Frequent, deep sighing is a characteristic symptom.

\section{IN OXEN, SHEEP, \&C.}

In other animals, sheep especially, diseases of the liver are more common than in the horse. Abscesses 
and great alteration of structure have been met with, although nothing very much amiss was observed during life.

.The symptoms and results are substantially the same as in the horse.

\section{Treatment.}

Aconitum should be given when the fever is high, the skin hot, the tongue furred; and when there are thirst, restlessness, and pain on pressure in the region of the liver.

Mercurius is indicated when the whites of the eyes, and the skin generally, present a yellow color; when the tongue has a yellowish fur, and the evacuations are knotty, and clay-colored.

Nux Vomica is suitable against great tenderness on pressure in the hepatic region, vomiting, thirst, highcolored urine, costiveness.

Arsenicum is especially indicated after the disease has made some progress, and when typhoid symptoms are appearing, such as vomiting; offensive, blackish diarrhœea; cold legs, great prostration; weak, small, irregular pulse. It is also suitable in the chronic form of hepatitis, when the liver is enlarged, the urine scanty, and dropsy of the belly present; or when the disease occurs in connexion with mange, or other skin eruption.

Digitatis is an important remedy against intermittent, and frequent pulse; evacuations dry, and ash-colored; urine thick and brown; pain in the side, \&c.

Podophylin should take the place of Mercurius for the same symptoms, when that drug either fails to do good, or has already been given in too large doses.

Doses.-20 drops for horses and oxen; 10 for sbeep and pigs. 


\section{3.-Flukes in the Liver-Rot.}

\section{IN OXEN AND SHEEP.}

The fluke-worm, or distoma hepaticum, is found in the liver of sheep much more frequently than in that of oxen, and is associated, in the former animals, with a peculiar condition of the systern, which one writer quaintly calls " a direful ruin of the general health and constitution." This so-called fluke disease has proved most destructive to sheep in certain years, notably in 1860 .

Rot is most prevalent in low, damp, marshy, flooded pastures, with rank grasses. The eggs of the perfect worm are found abundantly in the sheep's liver; they pass along the gall-duct into the bowel and thence are discharged. They are washed into pools oi brooks by rain, or fall on the grass; in either case, many die, whilst others are hatched. The embryos are eaten by mollusks and the other inhabitants of pools, and sheep swallow the latter. The immature worm then reaches the liver of its host, where generative organs are developed, and eggs are laid for the next generation. Such is a brief sketch of the changes which these worms are believed to undergo.

The symptoms of rot generally come on gradually. In the former case, the sheep appear dull and listless, and slowly lose flesh and strength. The membrane of the eye and the skin presents a yellow tinge, more or less deep. When patches of wool drop off, this color of the skin is well seen. Both the pulse and breathing are increased in frequency. Quick, short breathing, and slight, irritating cough ordinarily attend those cases that have worms (strongyli) in the air-passages, as well as 
flukes in the liver. There is great thirst and loss of appetite. At a later period, dropsical swellings make their appearance in different parts-in the belly, causing the belly to become swollen and the back stiff, and also about the throat. At this stage purging is a frequent symptom. In bad cases, the weakness gradually increases, and the animal becomes dull and stupid, and dies before long.

\section{Treatment.}

In this disease change of diet is of prime importance. All affected animals should be at once removed to a better pasture, and be fed on nourishing food, such as peas, corn, beans, \&c. A supply of common salt is also advisable. Purging is hurtful, because it increases the already existing weakness.

The best medicines are Arsenicum and Ferri Sulphusgiving them simply for a week at a time, or alternately.

Doses.-Two grains three times a day. .

\section{4.-Splenic Apoplexy.}

\section{IN OXEN AND SHEEP.}

This is the current name for a disease, very common amongst ruminants, which consists of congestion and extravasation of blood in the spleen. It would appear to be a pure blood disease, the spleen being the organ chiefly involved in the local destructive effects.

In principally attacks high-conditioned animals, or others that have been turned from poor into rich pastures. It is also rife where drinking water contains organic impurities, and on badly drained lands in hot seasons.

Serious and even fatal results.arise when pigs and 
other animals swallow the blood, flesh, or offal of cows or sheep that have died of this disease.

The disease comes on suddenly and without warning, and often destroys life in a few hours-from three to eighteen, in the majority of cases. At first the animal appears to be excited and the eye-balls protrude. Presently, it is uneasy and restless, and rnanifests the usual symptoms attending pain in the belly. The urine and fæces are often tinged or mixed with blood. The back is arched and the animal remains fixed in one position. The breathing is short and quick; the pulse quick, and, in the last stage, weak and imperceptible. Before death the animal falls down; a reddish, frothy discharge flows from the nostrils; the muscles twitch; the legs and ears are deadly cold; the teeth are ground; and the animal moans or bellows.

In sheep, the symptoms are identical with those just described as occurring in oxen and cows.

\section{Treatment.}

In order to prevent the disease and to arrest its ravages, it is necessary to change the pasturage and drinking water, and to attend to drainage, \&c. In the medical treatment, small success attends any means whaterer. Dashing cold water over the body and causing the affected animals to be rapidly moved about, are said to be useful. The best remedies are:-

Ammonium Causticum in the early stage, when the balance of the eirculation is disturbed, and hlood herins to stagnate in the spleen.

Dose.-Mix one part of the strong liqnor with seven of water -of this mixture give 20 drops every half hour, or hour, until there is improvement. 
Arsenicum is of great service for purging, pains in the bowels, bloody discharges from the mouth, prostration of strength, \&c. ; but such symptoms almost invariably end in death.

China may be tried, should the case recur, and there be symptoms of enlarged liver.

Doses — Of the two last medicines, 20 drops, every 4 hours ; for sheep, 10 drops. 


\section{CHAPTER VIII.}

DISEASES OF THE KIDNEYS, BLADDER, ETC.

\section{1.-Nephritis-Inflammation of the Kidneys. IN ALL ANIMALS.}

Nephritis is an occasional, and at all times a most dangerous disease in animals. It may be caused by the presence of a stone; by various injuries, such as blows, sprains, \&c., on the loins; it may follow chronic diseases of the bladder, and the employment of cantharides and turpentine. Blisters consisting of cantharides are still largely used; they are really unnecessary in the cure of any disease, and are undoubtedly painful. They are also dangerous ; for the animal may use his tongue and swallow some of the blister, and nephritis may be the consequence.

The use of diuretic balls by grooms and others is also a very frequent cause of this disease.

In this disease, the pulse is hard and quick; the breathing hurried; the skin hot and dry; the mouth hot; the bowels costive ; the urine very scanty, passed in very small quantity with frequent urging, and sometimes mixed with blood or matter. There is also intense thirst. 
The attitude and behavior of the animal is almost characteristic. The hind legs are stiff and kept widely apart, and there is the greatest reluctance to move.When he is compelled to move, he does so in a stiffened, constrained manner, and may walk lame. The loins are hot, arched, and very tender, so that pressure causes flinching. At a later stage, in unfavorable cases, the breath and perspiration smell of urine, the animal becomes depressed and sleepy, and ere long falls down, struggles, and dies.

\section{Treatment.}

Throw up occasionally a glyster of hot water, give plenty of linseed tea or barley for drink, and apply warm fomentations, or a sheep's hot skin to the loins. The best remedies are the following:-

Give Aconite in the early stage, when the symptoms of fever are marked, such as, hot skin and mouth ; quick pulse and breathing; great thirst; urine scanty, \&c.

When, as is occasionally the case, the disease is traceable to bad food, such as mow-burnt hay, kiln-dried oats, \&c., give Nux Vomica, especially if frequent urging to pass urine be present, in company with indications of colicky pains, looking round to the side, \&c.

Camphor may have to be administered as an antidote, when the disease arises from spanish-fly, used in a blister or otherwise.

Cantharis is an excellent remedy in cases not due to that drug, when the urine is scanty and discharged in small quantities with urging efforts, and also when it is mixed with blood.*

* Refer to the remarks on " Fræmaturia.' 
Arnica may prove useful when the disease has resulted from injuries to the back; and Rhus Tox. when from sprains, \&c. Both may, in such cases, respectively, be applied externally in the form of a lotion.*

Mercurius Corr. is indicated in the advanced stages, when the disease has gone on to the formation of matter, or to enlargement.

Doses.-20 drops for horses and oxen; 10 for sheep and pigs.Each dose to be repeated every two, three, or four hours, according to the urgency of the symptoms.

\section{2.-Diabetes Insipidus-Profuse Staling.}

\section{IN HORSES, \&c.}

Here there is excessive secretion and discharge of urine. It is met with most frequently in horses; comparatively rarely in the other lower animals.

Musty or otherwise damaged oats and hay are very apt to bring on this disorder; and then it is associated with indigestion. In sheep, it has been ascribed to eating certain plants, including asclopias, vincetoxicum, anemone nemorosa, \&c. The use of diuretics, such as nitre, may induce it.

The symptoms present no difficulty. The animal eats less than usual, is soon tired, sweats easily, and is weak and dull. The skin is dry and rough. There is intense thirst, and a disposition to lick the wall and to eat all sorts of rubbish. The mouth is dry and clammy, the

* See list of external applications. 
tongue furred, and the breath offensive. The urine is perfectly clear, or more or less milky looking; it is passed frequently in enormous quantities, and is altered in its chemical composition. The animal rapidly sinks in flesh and strength, and, in rapid cases, attended with very profuse staling, dies from exhaustion in a few days or weeks; in slow cases, not before a few months.

Diabetes Mellites, in which sugar exists in urine, has been occasionally met with in animals, but it is much more rare than the disease above described. Both diseases are as yet but ill understood, and deserve further investigation by the veterinarian. In both, the general symptoms are the same.

\section{Treatment.}

Affected animals must at once be placed on different food and pasture. Good old hay in moderate quantities is the best. Carrots are good. Not much water should be allowed, and to that little, add pease meal, or flour gruel.

The most likely medicines to be of service are:Ascidum Phosphoricum, which should be first tried, when the urine is largely increased, and the animal weak and wasted.

Nux Vomica is indicated when; in addition to the symptoms above described, there are those of indigestion.

These two failing, Baryta Carbonica and Iodium should be administered.

Doses. -20 drops for horses and oxen. Repeat each dose every two, four, six, or eight hours, according to the severity of the symptoms. 


\section{Case. *}

The following case illustrates the symptoms and treatment of a common form of this disease :-

On August 16th, 1853, a horse belonging to Mr. John Hewitt, coach proprietor, Manchester, presented the following:

Symptoms.--Pulse 28 and weak; respiration normal; the whole body is very cold, the legs particularly so; the hair rough, unglossy, and staring; the tongue is of a dirty yellowish color, and some half masticated food remains in the mouth; the appetite is both impaired and depraved, for the animal manifests a predilection for dirty litter, and refuses to partake of good diet; frequent micturition; urine profuse, clear and limpid ; the bowels are constipated, and the fæces are enveloped in mucus.

To have 20 drops of Nux Vomica 1, three times daily.

19th.-Skin of the proper temperature and appearance; tongue clean; appetite much improved; fæces and urine natural, both as regards amount and character.

Continue medicine.

20th. - Convalescent. The horse has worked a job of sixteen miles.

\section{3.-Scanty Urine.}

\section{IN ALL ANIMALS.}

In a great number of diseases, the urine is scanty, as a symptom. It is also more or less diminished in hot weather, and when the animal is worked, or much exercised, because then a large quantity of the fluid of the body is carried off by the skin.

It occasionally happens, however, that the horee, for example, suffers from scanty urine and nothing more, attended with some more than usual efforts to relieve

"Quoted from "Veterinary Homœpathy Illustrated.." 
limself-a state of things which knowing grooms consider as requiring a "staling ball."

In such a case as this, instead of giving a drug that may set up an awkward amount of irritation, recourse should be had to Arsenicum or Bryonia, three times a day, until the above symptoms pass off:

Doses.--20 drops for horses and oxen; 10 for sheep and pigs.

\section{4.--Retention of Urine.}

\section{IN HORSES, OXEN, \&C.}

Here there is urine in the bladder requiring to be voided; but either the expulsive power is absent, or the natural channel is obstructed.

Retention arises from a variety of causes, the chief of which are:-Paralysis of the bladder, or spasm of its neck; stones lying at the neck of the bladder; enlarged prostate gland; stones or worms in the canal; contractions of this canal, \&c. Male animals are more subject to it than females.

The symptoms are:-First, Distention of the bladder; a state of things which any experienced person can satisfy himself of, by making the proper examination through the rectum or vagina; in a word, the bladder is found full of urine. Secondly, There is pain on pressing over the bladder in the pubic region, and dullness when percussing it. Thirdly, The animal lies, and shifts about from one place to another; when made to walk, he does so unwillingly, with back arched and straggling gait.Fourthly, There are frequent, strong, straining efforts to 
urinate, but inability to do so; these efforts gradually abate, as the bladder loses its sensibility. Fifthly, Not only is there inability to pass urine-retention of urine -but there is no power to hold it, and the fluid slowly dribbles away-incontinence of urine. This happens only when the resistence to escape at the neck of the bladder is overcome by the pressure of the fluid behind; showing that incontinence is, in a majority of cases, a symptom of retention.

In the horse, especially, this condition is apt to be regarded by inexperienced persons, as that of colic, from the circumstance of there being, in colic, diminished secretion of urine. Nevertheless, there are many cases of undoubted colic attended with frequent attempts to urinate, and the two diseases cannot, as some writers assert, be distinguished merely by that symptom.

\section{Treatment.}

The immediate danger is to be overcome by emptying the bladder by means of the catheter-an instrument which can be introduced without much difficulty in the horse and all female animals. In the ox it is impossible, owing to the curve in the latter's penis. Whenever the catheter fails, or cannot be employed, the urethra (urine tube) requires to be opened in the perincum. An operation is also required, especially in the horse, when the obstruction depends on stone.

In other cases, the following remedies are of service:Nux Vomica, when the bladder is paralyzed or weak, and when the retention arises from constipated bowels.

Cantharis, when the urine is bloody, and passed with strong, straining efforts, in small quantities. 
Belladonna, when the retention appears to arise from spasm of the neck of the bladder.

Doses.-20 drops for horses and oxen ; 10 for sheep and pigs. A dose every two hours.

Injections of warm water into the rectum are often valuable. Small animals should have a warm bath, but not too warm, and only for a minute or two.

\section{5.-Hæmaturia-Bloody Urine. \\ IN HORSES, OXEN, \&c.}

This condition is of frequent occurrence amongst the lower animals. It arises from blows, sprains, and any manner of injury involving the region of the kidney, caused by overloading, jumping, \&c. It is a common symptom of stone in the kidney and bladder, of various diseases of these organs and their appendages, and of purpura hæmorrhagica; and frequently follows calving.

In hæmaturia consequent upon strains, injuries, \&c., we find pain in the loins when they are pressed upon, some degree of feverishness, and discharge of clotted blood, alone or along with the urine. This form is very apt to recur, especially if the affected animal return to work too soon, or be overworked.

Another form of hæinaturia is that which prevails extensively in certain districts, affecting all herb-eaters more or less, and said to be produced by eating various hurtful plants and young trees, including arnica, aconite, digitalis, genista hispanica, \&c. At first, the urine is scanty and reddish; subsequently, bloody and discharged with ardent pain. The pulse is quickened, the 
appetite lost, the mouth hot, and the coat staring-all symptoms of febrile excitement attendant upon active congestion of the kidney, which may run on to inflammation and abscess.

A third form is met with in animals pasturing on poor, and badly-drained land, and prevails most in wet seasons. Stall-fed cattle, living on turnips, are very subject to it; it is rarely observed where rich fodder is provided. It constitutes what is commonly called Red Water, so often prevailing amongst cows; a disease characterized by debility and poorness of blood. This disorder begins slowly with loss of appetite, staring coat, dullness, tender loins, \&c. Then the urine is observed to be higher colored than usual, and the milk is often tinged red. The animal becomes weak, the breathing quick, the pulse small and feeble, and the legs, ears, and horns cold. Cudding and secretion of milk are suspended. The membrane of the eye is whitish, or, when jaundice co-exists, tinged yellow. Generally the bowels are very costive, sometimes relaxed. At a later period, the urine becomes still more strongly colored red, and may become even black, strength and flesh fast decline, the eyeballs sink in their sockets, and death soon occurs from exhanstion.

\section{Treatment.}

In the first form, where the bloody urine has resulted from injuries, blows, \&c., the animal must be kept at rest, and have cold water injections thrown up the rectum, or cold water poured on the loins. Arnica should be administered inwardly, and Arnica Lotion* applied externally. When it is merely a symptom of stone, or

* See list of external applications. 
of diseased kidney, \&c., the major disense must be treated.

In the second form the animal must be removed from the injurious pasture and fed on soft fond, including linseed tea.

Aconite should be given when there are symptoms of feverishness, such as hot mouth, quick pulse, diminished secretions, etc.

Cantharis, when, in additicn to there being blood passed alone or mixed with urine, there are forcing efforts to pass it, attended with pain.

Teretintha is sometimes of greater service than the last medicine for the same symptoms.

In the third form, stop turnips and give generous diet, plenty of linseed gruel, etc.

The best remedies are Cantharis and Terebintha.

Doses.-20 drops for horses and oxen; 10 for sheep and pigs. Give a dose every three hours.

\section{6.-Cystitis-Inflammation of the Bladder. IN ALL ANIMALS.}

Amongst the causes of cystitis may be enumerated: exposure to damp and cold, injuries, extension of inflammation from the kidneys, the irritation produced by a stone, by cantharides, etc.

The symptoms are frequent pulse, restlessness, and general constitutional disturbance. There is trembling of the hind legs, attended with frequent efforts to void urine, which is either not discharged at all, or passes away drop by drop. After the evacuation of the urine, the pain subsides for a time. When the animal, during 
the pain, looks round to his flank, etc., an inexperienced person may think that the case is one of simple colic; but the evident and unmistakable urinary difficulty will make the natnre of the case plain. That portion of the belly in which the bladder is situated is hot and tender, when examined with the fingers. When the bladder is examined through the rectum or vagina much pain is felt. The urine varies in its appearance, being either clear, or mixed with either mucus, sediment, or blood. If the disease go on, other symptoms make their appearance. The bladder, previously so irritable that it contracted with the greatest force on even a few drops of water, now loses its power, and the urine gradually accumulates within it. The muscular wall is in fact paralyzed. When the coats of the bladder are so much stretched that further dilatation is impossible, the neck of the bladder yields and the urine dribbles away involuntarily.

\section{Treatment.}

When there are quick pulse; frequent desire to urinate, discharge of scanty, bloody, turbid urine; pain on pressure in the region of the bladder, give Aconite.

When the urine is discharged drop by drop with great force; the pain increased during the act of passing it; the region of the bladder painful and distended, give Cantharis.

Nux Vomica is another good remedy for the last mentioned symptoms, and may be used in the rare event of Cantharis faillng.

Linseed tea, etc., should be freely administered.

Dosks.-20 drops for horses and oxen; 10 for sheep and pirs.

Repeat each dose every two or three hours. 


\section{7.-Calculi-Stones.}

\section{IN ALL ANIMALS.}

Stones met with in the urinary apparatus are named according to the particular part in which they are lodged.

1.- In the Kidney.-Stones in one or both kidneys have been found in all animals, the horse and pig in particular. Tenderness of the loins, discharge of blood, and attacks of pain with manifestation of colicky sufferings are the chief symptoms, but they are not characteristic.

2.-In the Ureter.-Stones sometimes pass from the kidney into the tube leading to the bladiler, but this is rare, owing to the horizontal position of the ureter. In its passage, most severe pain is excited. If it should be arrested in its course, the urine collects in the kidney and seriously injures that organ. This is specially the case in the pig.

3.- In the Bladder.-The chief symptoms are:-straddling manner of walking, the hind legs separated, frequent efforts to pass urine, stoppage of urine, bloody urine, dribbling, and detection of the stone by passing the hand into the rectum to examine the bladder-into the vagina in female animals; or by using a "sound," when this is feasible.

4. - In the Urethra.-In male animals, stones frequently lodge in the tube for the passage of urine outwards from the bladder, and, obstructing the flow of urine, set up colicky symptoms. Sheep and rams are especially subject to this.

5.-In the Prepuce, or Fore-skin.-Most common in horses and pigs, and in lucing difficulty in urinating. 


\section{Treatment.}

For the attacks of pain attending stone, and for urinary difficulties so often met with from stone, treat as directed under "Colic," and under individual heads in this chapter. Stone lodged in the bladder and urethra must be removed by operation; otherwise, a radical cure cannot be effected.

\section{8.-Dysuria-Difficulty in Urinating.}

\section{CASE IN HoRse.*}

In July, 1851, Mr. Tinsley, of Warrington, consulted me respecting a valuable carriage-horse. The following symptoms were furnished to me: In external appearance the animal is well, and eats and works as usual. He has, however, the greatest difficulty in urinating; places himself in the necessary attitude, and strains frequently and with considerable force, for upwards of an hour-when the urine comes freely and he is at ease; sometimes a few drops are spirted out. Allopathic drugs have been given wholesale for a long time, without doing the slightest good.

I prescribed 20 drops of Aconite, 1st dilution, in the morning, and the same of Arsenicum, at night.

At the end of the week I received information that my patient was very much improved, and I was asked for more of the same medicines.

Six weeks later I was informed that owing to the owner's absence, the medicines had not been continued, and the horse was occasionally affected with slight symptoms of the old complaint.

The medicines were again given, and a cure effected in another week.

A few days ago-I now write on September 14th, 1858-Mr. T. told me that his horse had been perfeetly well ever since.

*Quoted from Brit. Journal of Hom. for 1858 . 


\section{CHAPTER IX.}

\section{DISEASES OF THE GENERATIVE ORGANS.}

\section{1.-Abortion.}

No domestic animals perhaps require nearly so much or such constant and immediate care during pregnancy as cows, for none are so liable to incomplete, premature, unproductive, dangerous, or fatal deliveries. It might almost be said, that irregularities in gestation are the more probable contingencies. Abortion, or, as it is technically called, "stinking," abruptly terminates the pregnancy of cows under an endless variety of circumstances.

It may be termed a species of epidemic disease spreading unaccountably amongst cattle, and continuing to affect them for years. In the majority of cases, however, it is rather of accidental or other local origin, than attributable to epidemy. Nor even when introduced amongst particular herds by the admission of a strange cow previously subject to abortion, can it properly be termed epidemic, for even then the original cause may be traced to a particularity, not a generality.

It may very properly be termed an endemic disease, 
affecting all the cattle in particular localities, and arising in such cases from the stimulating, acrid, or other mischievous nature of the food, from the peculiarity of the water habitually drunk by the cattle; such, for instance, as the presence of chalybeate infusions; from the peculiar nature of the atmosphere, \&c.

It may decidedly be looked upon as sympathetic, being induced even by the presence of a cow addicted to abortion, by the odors emanating from the discharges accompanying or following it, and many similar causes. Wherefore every precaution should be taken to neutralize odors, to cleanse the spot, whether within or out of the stable or shed, to remove all other cattle to a distance, and to sink the abortive calf deep into the ground, and in some spot entirely unfrequented by the cattle, such as a wood or garden, as far from the range of the pastures and cow-houses as possible.

There is no question but that abortion is hereditary in the few instances in which a cow, prone to slinking, has succeeded in rearing her calf. Wherefore, we should strongly discourage the breeder from ever rearing such calves (if female) for milch cows, and recommend him either to dispatch them for slaughter, as calves, or to rear them for fatting only.

Again : abortion may be considered hereditary in another respect, as dependent upōn hereditary defects of constitution. Wherefore, a cow of general unhealthy habit, and especially if unkindly and indicative of the presence of consumption, should never be appropriated to purposes of breeding.

Abortion may justly be held to be periodical or rather atmospheric, for it is very well ascertained that it becomes 
peculiarly prevalent during certain seasons, under particular conditions of weather, \&c.

Abortion is often, and perhaps most frequently, denominated as accidental, and may be occasioned by any circumstances which tend to interfere with, or to interrupt, the regular operation of any particular function; for it must not be forgotten that the cow is of a peculiarly hysterical temperament, that is to say, that anything which affects the beast in ever so slight a degree, is likely, especially during pregnancy, to react upon the womb, and may sometimes affect a future pregnancy when the cow is not actually in calf at the time. Amongst these general accidental causes of abortion, we may number fright (from any cause) ; excess of food; over-stimulating food; plethoric condition arising out of food or confinement; want of fresh air and free scope; foul, close, unhealthy cow-houses; deficient or unwholesome food; acute diseases in general, especially those of an inflammatory nature; sudden changes of pasture or stall food; as also mechanical injuries, and intercourse with the male during pregnancy, \&c.

\section{2.-Premonitory Signs of Abortion, Precau- tions against it, and General Treatment to Follow it.}

Cows which have once slinked a calf should be constantly watched, especially about the twentieth week of pregnancy, and from that time to a period varying from seven to four weeks before the estimated period of parturition. By observing this precaution, proper general 
treatment and proper remedies may be resorted to in time to arrest any untoward consequences.

The approach of abortion will be aistinguishable in the gait and movements of the animal, which will have a tendency to retain the same position, whether recumbent or erect. The movements of the calf in the womb will be less distinctly observable, and whereas, under propitious circumstances, these movements would be palpable externally to the eye, it will be necessary, on the near approach of slinking, to place the palm of the hand upon the right flank, towards the hind quarters, in order to distinguish them; the cow will feed but irregularly, evidently without relish, insufficiently, and without ruminating; the belly will droop, lose its sideway fulness and roundness, and become lank. This last will more especially be the case when the calf is dead, under which circumstance all motion will have ceased; the pace will be uncertain and wavering; the milk will fail; and, as the prernature labor becomes imminent (and almost always unavoidable, but invariably so when there are symptoms of decomposition), sounds indicative of pain will be emitted ; the respiration becomes catching, interrupted, irregular, and difficult; a discharge either of a yellowish or darker and bloody hue exudes from the parts, which, if very offensive, indicates the decomposition of the contents of the womb; and the pulse indicates considerable prostration (much resembling that attendant upon the low species of nervous fever), and is frequently very irregular; sometimes even barely perceptible.

The homœopathic treatment is especially adapted to approaching abortion; the homøopathist possesses a pe- 
culiar advantage in having direct specifics to employ against the disease, whereby, even in cases in which he might mistake the precursory for more imminent symptoms, his treatment will have been as apposite to prevent, as it would later be to facilitate, the deposition of the calf.

The after-treatment of cows, which have slinked their calves, should consist in the most expeditious removal of all offal, in treatment to promote the ejection of the after-birth, and if necessary, of mechanical means for this purpose; in thoroughly cleansing the animal and its litter, and in allowing the free circulation of fresh air. The only food allowable immediately before or after abortion, should consist of thin gruel, slightly warm.

The subsequent treatment of all cows, which have prematurely deposited their calves (except in some rare cases in which mechanical injuries are purely the cause of the accident), may be summed up in four words,get rid of them!

\section{3.-Parturition; Its Treatment.}

The delivery of the calf either takes place in due and proper course, and without difficulty, or it is impeded, painful, slow, very difficult, or even totally arrested. In the former case, little or no interference is requisite, other than to watch the progress of the labor, to be at hand at the moment of delivery, and to render every assistance to the cow and calf, as well as to see that the afterbirth is properly ejected. If the latter is the case, however, and the labor has continued ineffectually for nine or ten hours, medicinal, or even mechanical, interference becomes necessary. 
The removal of the after-birth will sometimes require medicinal, or even mechanical, interference, but this is rarely the case. As soon, therefore, as the labor is safely brought to an issue, we should content ourselves with placing the calf immediately within reach of the mother, and with seeing that a thin mash of tepid gruel is given to her. The suckling of the calf should be encouraged and facilitated (unless there be profuse and prolonged discharges of blood, when it must be withheld); and if the calf does not suck freely, we should resort to the expedient of drawing off the milk. But, under any circumstances, we should not remove the calf from the mother without sufficient reason. The process of licking, to which the cow submits its young, is alike advantageous to both. In the event of profuse and prolonged discharges of blood following delivery, we should have recourse to the medicinal treatment hereinafter recommended under the head of "Parturition."

Approaching parturition is indicated by the following symptoms, and, when these are present, the cow should be conveyed to a place where she will not be exposed either to the inclemency of the weather, or to accidental intrusion or interference, quiet being essential to her.Cold water should be sedulously kept out of her reach, because, if at hand, the febrile action, which in all cases, more or less, accompanies calving, will induce an inclination to drink it, and the pains may thereby be so far modified as to render them inadequate to the expulsion of the calf. We may allow harmless warm drinks, such as unstimulating mashes, gruel, \&c.

The premonitory symptoms are generally these: Great uneasiness, gradually increasing, the cow constantly 
changing position, as, for instance, laying down as soon as it has got up, and vice versa; the discharge from the passage, such as has aiready been described under the head of "Abortion;" the sudden distension of the udder,- the appearance of the belly being such as has already been described; a peculiar, slow, periodical moan, which is only heard on the approach of or during labor; the swelling of the parts, and afterwards a peculiar muscular action of a forcing description, which regularly accompanies the recurrence of the pains. As labor approaches or begins, the pains become more regular, evidently more violent and quicker in succession, and the pulse and respiration quicker. During the whole of this time we should abstain from meddling with the beast; but when, from the presence of certain discharges, or from the length of time since the commencement of the labor, we have reason to believe that the delivery should be at hand, we should proceed to examine the cow, for the purpose of ascertaining the position and advancement of the calf, and whether or not there be any hinderance in the construction of the cow which will impede its progress.

The position or formation of the calf is either natural or unnatural.

1st. In its natural position, it should be extended on its stomach, with the back upwards, (relatively to an erect position of the cow) and it should advance in the passage by the fore legs projecting forward, the head being also stretched forward, so that the nose rests upon and between the knees; but not so as to project below them.

If there be nothing in respect of the conformation of 
the cow, to interfere with ready delivery, and if the parts are properly dilated, whilst the labor-pains (as indicated by the excessive pressure and resistance offered to the hand) are strong enough to expel the calf, it is better to let nature do its work.

$2 d$. The position of the calf being unnatural consists in its being different from that above described.

It is sometimes reverted sideways ;

Sometimes on its back, with the belly turned upwards, relatively to the erect position of the cow ;

Sometimes with the nose so sunk as to offer an impediment to its passing, \&c.

In the majority of these cases, medicine will not suffice without the application of mechanical resources; and these mechanical resources sometimes extend to the partition of the calf, for the purpose of removing it by pieces, which is always justifiable when the calf is dead.

The formation of the calf may be such as to preclude the possibility of delivery, when surgical resources can alone be of any avail. It has even been thought necessary to open the side of the cow for the extraction of the calf: the more common method is to extract the calf by pieces.

There may be impediments arising out of the construction, malformation, or accidental contraction, \&c., of the parts in the mother. In the majority of these cases mechanical means become requisite.

If the labor-pains become weakened or suspended, the treatment hereinafter directed, under the head of "Parturition," must be resorted to. It is obvious to every one who pays the slightest attention to these matters, that both the cow and calf are prejudiced by the undue 
extension of the period of labor ; and consequently anything that tends to protract the expulsion of the calf should be obviated, as soon as possible.

If the labor pains continue unabated, and strong enough to expel the culf, during more than from ten to twelve hours, we may infer that there is some mechanical obstruction, either in the cow or calf, and must proceed accordingly, as before directed.

\section{4.-Mechanical Means for Assisting Delivery.}

The hand must be oiled and carefully inserted, so as to ascertain the position of the calf; and if the general position be found as it should be, with merely a slight depression of the head, it may be found sufficient to raise the head, and place the head and fore feet so that all difficulty is removed. In most cases it becomes necessary to attach a cord to the fore legs and jaw, wherewith to draw the calf forward simultaneously with the propulsion given by the labor-pains. The same means are often found sufficient when the difficulty arises from malformation in the mother. When the position of the calf is otherwise unnatural, we must endeavor to alter the position, agreeably to the description of natural position, given above. The more complicated cases, involving serious operations, are beyond our province.

\section{2.-Flooding after Delivery.}

In all cases it is usual for some amount of blood to be lost after the young is born, without any injury result- 
ing to the parent animal and without the necessity of arresting the discharge.

When the womb does not contract firmly, as it ought to do, after delivery, a considerable quantity of blood may flow into its cavity and externally. Injuries to the womb or passage inflicted during delivery, particularly when the process has been difficult, and when assistance has been required, may give rise to excessive bleeding.

\section{Treatment.}

In the last mentioned instances, after the expulsion of the after-birth, the treatment consists in keeping the animal quiet in a cool, well-ventilated place; in applying a bandage tightly around the belly; in pouring cold water from a height, and throwing injections of cold water up the rectum or vagina; and in giving the medicines recommended for "Abortion" - the remarks on which should be referred to.

\section{6.-Retained Placenta.}

Naturally, the womb expels the after-birth by its own contractible power. When this fails, and the after-birth does not come away, it is usual, if it be in the womb, to introduce the hand and thus remove it, or, if it be in the vagina (passage from the womb outwards) the same means can be more easily employed. Injections of warm water are sometimes used.

In my experience, two medicines, viz., Pulsatilla, or, this failing, Secale, render all manual measures unnecessary in the great majority of cases. I speak with more particular reference to cows. China is also of service.

Doses.-20 drops for mares and cows; 10 for sheep. 


\section{Case.-Retained Placenta-Irritative Fever.}

March $27 \mathrm{tb}, 1859$, I was called to see a cow belonging to $\mathrm{Mr}$. John Greenwood, coach proprietor, Manchester. The cow was bought on the 23d, from a Yorkshire dealer, had traveled a long distance, and calved on the 26th. This morning she began to ail, and a cow leech was fetched to give her an "opening drench." I found the pulse 110, small and thread-like; respiration quickened; no appetite; the cow was in a drowsy, sleepy state, and although able to rise, did so with some difficulty, and was evidently weak in the back; there was very little milk and the cow bad not cleansed. In my absence, Aconitum and Belladonna were sent, and I ordered them to be continued. On the 28 th the pulse was 98 , the breathing more tranquil, the appetite improved, rumination returned, the sleepiness gone, and the placenta discharged. On the 30 th the cow was quite well.

\section{7.-The Earliest Treatment of Calves.}

Apart from the more special directions for treatment, it may be very properly mentioned in this place:First, that the navel-string should be examined, and proper bandages applied if the hemorrhage continue. Secondly, that, above all, the calf should (unless there be physical impediments) be allowed access to the first milk, than which nothing will more surely modify the first irregularities of the digestive functions. Thirdly, that the treatment of the calf should be incidental to that of the mother, for whatever will tend to injure the mother, during the first few weeks after delivery, in particular, will almost inevitably recoil upon the calf. Every precaution should, therefore, be taken to promote the secretion of good and abundant milk; all regulations with re- 
spect to cleanliness, air, and the like, should be strictly observed; and the first indications of derangement in the cow should be speedily obviated by appropriate treatment. Fourthly, that in the generalities, the same precautions are observable, with respect to the calf individually. Fifihly, that as soon as the calf has sufficient strength to follow the mother about in the pasture, and power, as well as disposition, to gambol, it will be far more consistent with common sense to allow it to remain with the mother, and freely to seek the udder, or to ramble unrestrained, than to keep it pent up in a close and unhealthy cow-house. It is a great mistake to suppose that the unlimited access of the calf to the udder, either deteriorates or lessens the supply of milk; the fact is quite the contrary, if the food be of a quality and abundance consistent with the requirements of an animal giving suck.

The period of weaning varies so much, according to the convenience of the owner, and is so frequently set aside altogether, by the slaughter of the calf, that it were difficult to set any precise limit for the guidance of the reader in this respect. It is clear, howerer, that, if it be intended to rear the calf, much must depend upon its strength and capability of subsisting independently of the mother. The advancement of the teeth is an important point whereby to judge of this; and it is not very difficult to discern whether the calf readily and easily partakes of general food. If it be intencled to breed again from the mother, it is natural enough that she should be separated from the calf, already sufficiently advanced to do without her support. 


\section{8.-Metritis-Inflammation of the Womb.}

\section{IN ALL ANIMALS.}

Of all the domestic animals, ewes are the most subject to this disease. It arises in connection with the process of delivery, and when the afterbirth is retained. Usually, it comes on in from four to eight days after delivery.

The symptoms begin with feverishness, such as hot mouth, checked secretions, quickened pulse, \&c. These are presently followed by straining and discharge of fluid from the womb; this fluid may be brownish, and even black in color. The loins and abdomen are tender to the touch, and the vagina hot. The bowels are generally constipated. In favorable cases, these symptoms gradually yield to treatment and recovery takes place; in unfavorable, the pulse and breathing increase in frequency, the discharge becomes offensive, and fatal sinking comes on.

\section{Treatment.}

When the bowels are much constipated, throw up a clyster of hot water, and give a few doses of Nux Vomica.

Injections up the passage are mest important aids to recovery in this disease, by removing the discharge, which, by accumulating, decomposes and does harm.When the discharge is offensive, Condy's Fluid should be added to the water.

In addition, give one or other of the following reme dies, according to the subjoined indications:-

Aconite for the symptoms of fever, such as hot skin and mouth, quick pulse, \&c.

Pulsatilla if there be reason to believe that a portion of the after-birth has been left in the uterus. 
Crocus is indicated when the discharge is dark colored. Arnica is required when the disease follows a difficult labor, and assistance has been employed by the hand or instruments.

Doses. - 20 drops for horses and oxen; 10 for sheep and pigs.

\section{9.-Discharge from the Vagina.}

\section{IN ALL ANIMALS.}

An increase of the natural mucus which is secreted in the passage of female animals may arise from several causes, including injuries, relaxation of the mucous membrane, inflammation of the same, from a condition of general ill health, \&c. The disorder in such cases would be called LEUCORRHÆA, or whites. A discharge of white, curdy mucus is a common symptom of chronic inflammation of the womb.

Cows are also subject to a disease arising from contact with the bull's instrument in a state of inflammation, attended with an irritating discharge. A considerable discharge of matter flows away, and the urine is passed in small quantities and with evident pain. The parts, on examination, are found to be swollen, red and painful. This disorder has been called GoNoRRHEA, or Clap.

\section{Treatment.}

The parts must be frequently bathed with luke-warm water and kept perfectly clean. An injection of Chloride of Zinc, one ounce, dissolved in two gallons of soft water, should be thrown up three times a day. When the discharge depends on relaxation, an injection of 
Hydrastis may be used; one of Calendula when there are wounds, or ulceration; and one of Iodine if there be much offensive discharge.

In addition, give Cantharis when it is evident, from the animal's manner and posturing, that urine is passed with pain and difficulty; and

Mercurius, when the parts are inflamed and tender, and the discharge thick and mattery.

\section{0.-Inflammation of the Prepuce.}

\section{IN BULLS, \&c.}

In this disease the fore-skin is inflamed and gives forth a mattery discharge. The corresponding disease, which is said to excite this, is described under the head of "Discharge from the Vagina," as it occurs in cows. On examination, the sheath and penis are found red, and swollen, and the fore-skin covered with matter. Great pain attends the act of urinating.

In some cases, the canal is the seat of inflammation, and then the disease would be called "clap."

\section{Treatment.}

Apply the same lotions, and give the same medicines as are directed above for "Discharge from the Vagina."

\section{1.-Garget.}

A disease to which cows and ewes are exceedingly subject, and which requires great care and attention. It consists of a species of inflammation and induration of the lacteal glands, and the interior of the udder and teats. 
SympToMs.-The first indication of the presence of this disease consists in the refusal of the darn to suffer the young to suck; and this is ever to be looked upon as a distinct symptom of derangement, because, besides the natural affection of the dam for its young, which prompts it to nurse its offspring, the painful distension occasioned by the rapid secretion of milk would, in the ordinary course, lead the dam to suckle the young for relief; upon examination, therefore, which should take place as soon as the above mentioned indication occurs, we shall generally find the udder exceedingly tender, of a burning heat, swollen, and knotty; the development of distinct hard lumps takes place in various parts of the udder, and the teats become likewise affected with these tumors or nodosities; in the earlier stage, the development of tumors, which is gradual and progressive, will not have taken place, and the general hardness, heat, tenderness, and swelling of the udder, \&c., will constitute the group of symptoms; later, considerable constitutional disturbance supervenes, and the pulse and respiration are powerfully affected ; the swellings or lumps in the udder become flabby and soft, owing to the progress of suppuration; sometimes proceeding to the development of large, ragged ulcers, or to the formation of permanent, hard, knotty tumors, with decrease and impeded discharge of milk; the milk is tinged with blood, and mingled with matter; the appetite fails, and rumination ceases.

Causes.-Excess of condition, especially at the first parturition; neglect during the latter period of pregnancy; careless and imperfect millsing, or cessation of milking for too long a period; the milk not being drained thoroughly off, so as to leave the more dense and coagu- 
lable portion of it in the udder, whereas it is coagulation of the milk, which is the immediate cause of garget; exposure in cold, bleak, damp, and marshy situations; and sometimes, also, the peculiar habit of the animal of exposing the udder to pressure when lying down, are also amongst the provoking causes; as, also, neglect in the treatment of the animal immediately after parturition (suffering inflammatory action to continue without due attention), or the effect of cold and moisture at that time.

\section{Treatment.}

Phytolacca.-Many homœopathic remedies have been advised for this disease; but they all are inferior to one which has long been known as a domestic remedy for cows affected with garget. And it is from the excellent effects of poke, or scoke, or "garget," as it is called in the eastern sections of the country, on cows, that it has come to be recognized as invaluable in similar disorders in the human subject. When a boy, I remember seeing my father slice up the green root of this plant, insert the slices in sorne pieces of raw potato, and give them to a cow whose "bag" was swollen and hard, and that she was thus cured. A similar experience is quoted by Dr. E. M. Hale: "When I was a student of medicine in my father's office, a neighbor had a valuable cow, which, after a clandestine confinement, was brought home from the woods with a most enormously swollen udder. It was hard as a stone, intensely hot, painful and sensitive, and not a particle of milk could be drawn. A dose of Epsom salts was administered, but after twenty-four hours the cow was worse than before. At this juncture an old woman of the neighborhood brought in a piece of a 
large, white, succulent looking root, which she called scoke, and ordered the farmer to cut a portion of it up finely and give the animal in some bran mash. Another, the larger portion, was made into a decoction, and the cow's udder washed with it frequently. The effect was magical! In less than twelve hours the milk could be drawn, the gland softened, and in a few days the morbid condition was removed."

This is a most remarkable homœopathic remedy, for it is capable of causing what it thus cures. Poke-weed, or Phytolacca decandra, known by its rows (racemes) of purple berries, grows in most parts of the United States, and constitutes one of the principal of the famous Indian medicines.

Dose.-Those who cannot readily procure the fresh root may use the mother tincture in 10 drop doses, in a little meal, once in three hours, and bathe the udder as often, with a lotion made by mixing 2 teaspoonfuls of the tincture in 1 pint of soft warm water.

The Phytolacca is no less suitable for mares than for cows.

Should the disorder already have advanced to suppuration, and this not readily yield to the Phytolacca, select Silicea, Phosphorus, or Sulphur, according to the prevailing symptoms, and give six drops of the chosen remedy, night and morning.

\section{2.-Sore Teats.}

The soreness, cracking, excoriation of the teats, or even discharge of matter from the fissures developed about the teats, resulting from a considerable degree of irritation or inflammation, is of very common occurrence $7^{*}$ 
amongst cows, and when the cow betrays great pain, and is restive during the milking, without the evidence $\mathrm{o}_{\Perp}$ constitutional disturbance, the appetite and rumination being unaltered, it is very desirable that the teats should be examined and appropriately treated, otherwise constrained suppression of milk may ensue, and entail more serious consequences. The habit of impatiently goading the cow, which is restive during milking, under such circumstances, is exceedingly reprehensible, and is not unfrequently followed by decreased or deteriorated supply; sometimes by the admixture of blood with the milk, or even by the occurrence of severe constitutional disturbance. If, indeed, the excoriation be neglected, it will often result in garget, arising out of the suppression and consequent coagulation of the milk; or if it does not lead to such serious consequences, the yield of the milk will become permanently diminished, or a vicious habit of restlessness incurred, which will render milking a serious task.

\section{Treatment.}

This is very simple and effectual. Wash the sores with tepid water, and keep the part thoroughly clean.Then apply Calendula Lotion, at least four times a day; and this failing, Sulphurous Acid Lotion. If the teats are so painful as to prevent milking, the milk must be removed by introducing a teat-tube into the teat. By this means the milk will drain off, and the evil effects of its accumulation be prevented. If the udder should become inflamed, consult the remarks on "Garget."

I use at the present time a salve, composed of 1 pound of fresh butter, 2 drachms tincture of Iodine, 1 ounce Origanum. Melt the butter, and mix well together. 


\section{CHAPTER X.}

\section{DISEASES OF THE RESPIRATORY ORGANS.*}

\section{1.-Bleeding from the Nose.}

This accident may happen to all animals, but is of most frequent occurrence in the ox, and next, in the horse. Usually it must be regarded not so much as a disease but rather as a symptom of different diseases, including glanders, ulceration in the nose, polypus, purpura hæmorrhagica and other blood diseases, \&c. It may likewise arise from injuries, over-exertion, \&c. The symptoms are sufficiently obvious. The blood is bright colored, except in certain blood-diseases when it is dark colored.

In horses severely galloped in hunting, blood may issue from the mouth also ; and if it be in large quantity, death may be instantaneous.

\section{Treatment.}

Where nose-bleed is symptomatic of disease, the latter must be treated; but in all cases when the bleelling is excessive, it demands exclusive attention for the time. Slight bleeding requires no treatment-it generally stopls

* Including the nose, nasal chambers, windpipe, air-tuhes, lungs, pleura, \& 
of its own accord. When this does not take place, treat in the following manner :-

Aconite is required when the bleeding attacks a horse in high condition, as the result of over-exertion, and when the pulse is full and quick, the breathing labored, the membrane of the eye injected, \&c.

Arnica is more suitable when the bleeding is the result of local injury. The injection of Arnica Lotion* may be restored to, in addition, in bad cases.

Pouring cold water on the head from a height may be advantageously employed.

The use of tight collars, by impeding the return of blood from the head along the jugular veins, may predispose to, and even excite bleeding from the nose. Of course, such a collar should be discarded.

For the treatment of bleeding from the nose dependent upon glanders, ulcers, purpura, the reader should refer to the observations on this disease in other parts_of this work]

\section{2.--Nasal Catarrh-Coryza. IN HORSES.}

This disease consists of inflammation of the nose, as well as of the maxillary and frontal sinuses, which communicate with the nose.

It arises from exposure to variations of temperature and to damp and cold; and from bad ventilation, foul, damp stables, \&c. It begins with sneezing, some feverishness, swollen eyelids, red eyes, \&c. ; these are present-

* See list of local applications. 
ly followed by a thin discharge which irritates the part over which it flows. Subsequently, the discharge becomes thick and mattery. The feverishness now begins to decline, the quantity of discharge decreases, the appetite returns, and health is speedily re-estatablished. In bad, neglected, ill-treated cases, however, especially in a weakly horse, the symptoms take on the character of nasal gleet. Many cases are accompanied by sore throat, cough, and pain and difficulty in swallowing, from the inflammation extending to the throat.

\section{IN OXEN, SHEEP, \&C.}

In young oxen and cows, coryza is sometimes met with of a very severe form. It begins with the ordinary symptoms of nasal catarrh; the discharge, however, is bloody, mattery, and offensive. The nasal membrane in the last stage sloughs off; the horns and hoofs also drop off. There is great prostration throughout, and convulsions very generally come on immediately before death. This disease has been termed malignant coryza, and also ox glanders.

In the pig, the general symptoms are identical with those of the same disease in other animals.

\section{Treatment.}

Place the animal in a clean, well-ventilated box, clothe him warmly, and feed him on mashes for a few days. In order to have the nose cleaned out, cause him to breathe the fumes of vinegar by pouring some of the fluid on a red hot brick placed in front of his head on a shovel. This will make him snort and snceze, whereby a large quantity of matter will be expelled. The fumi- 
gation should be used at least night and morning so long as the discharge is copious.

The best remedies are :-

Aconite for dryness, stuffing, and redness of the nose; quickened pulse and breathing; hot and dry mouth; thirst and loss of appetite - the symptoms, in short, which denote the febrile state.

Belladonna for swollen eyelids, red eyes, sore throat, pain in swallowing, and flow of tears.

Mercurius for sneezing, free flux from nose, coughing and sore throat.

Arsenicum, especially if the discharge should continue in spite of the preceding treatment, and the disease threatens to lapse into nasal gleet; it is especially indicated when the horse is weak and off his feed; when the attack follows exposure to wet and cold, etc.

Dosfs.-20 drops for horses and oxen; 10 for sheep and pigs. One dose every two or three hours.

Carbolic Acid, ten drops three times a day, is a good remedy.

\section{Case of Catarkh in Pony.}

On June 20,1861 , I was requested to visit a pony, the property of His Grace the Duke of Northumberland. This pony was brought from the country to remain for the London season. In a few days it commenced to cough; was off its food, and had difficulty in swallowing; had muco-purulent discharge from the nose; pulse 60 , and rather feeble ; respiration 16 per minute; nasal membrane vascular; coughed when the throat was pinched; costive, and the frces thickly coated with mucus; urine scanty, and rather highly colored.

Treatment.--To have Belladonna and Mercurius v. alternately every three hours.

21st.--Slight amendment. Continue medicine. 
23d.-Pulse 46, and otherwise improved. Continue medicine every four hours.

25th.-Further improvement. Continue medicine.

27 th. -Well.

\section{3.-Nasal Gleet.}

\section{IN HORSES.}

All chronic discharges from the nose are spoken of together under this one name, or as constituting chronic nasal catarrh. The term, however, if used correctly, should be limited to those cases in which the mucous membrane lining the nose and its communicating chambers and windings, is in a state of chronic inflammation, attended with discharge of mucus, or of mucopus. Nasal gleet, in this sense, is usually the result of the acute form already described.

The discharge consists of mucus, or a mixture of mucus and matter; it is whitish, yellowish, or greenish ; it varies much in quantity, and is constantly flowing, although increased by sneezing and snorting; it is of a uniform consistence, sometimes partially clotted and lumpy; and it may issue from one, or from both nos: trils. The membrane of the nose presents an unhealthy leaden color. The gland under one or both jaws is slightly enlarged, it may be tender to the touch. In addition, the coat is staring, the appetite poor, and the strength more or less reduced.

\section{Treatment.}

On this point, I cannot do better than quote the account of two cases first published in "Practical reply to Sir B. Brodie's Letter :"- 


\section{Case I.}

On 14th of October, 1861, admitted a bay carriage-horse, the property of — Hamper, Esq., of Wandsworth. This horse has been under allopathic treatment for two months, aud daily getting worse. The veterinary surgeon recommended him to be killed as incurable.

Symptoms.-Copious discharge from both nostrils, adhering like glue; the whole of the nasal mucous membrane vascular; submaxillary glands enlarged; rough, harsh, unhealthy coat; low in condition, and feeble; bowels costive; urine high colored; pulse 44 , and feeble; bad appetite, \&c.

Treatment.-To have Hydras C., 20 drops, 1st dilution, three times a day, and the nostrils to be fumigated with a solution of the same drug.

15th.-The discharge from the nostrils not so copious, and the membrane not so vascular; pulse 40, and strong; appetite better. Continue medicine.

16th.-No discharge from left nostril ; slight watery discharge from right; pulse normal; nasal membrane nearly of its natural color. Continue medicine.

18th.-The watery discharge from nose quite ceased; gave four coughs this morning; to all appearance quite well ; no fumigations for two days.

19th.-Cured.

The Turkish bath also was given.

\section{CASE II.}

On the 6th of June, 1861, a bay carriage-horse, the property of the Right Hon. Lord Wharncliffe, was sent to me, having a discharge from both nostrils. This horse has been under allopathic treatment for a considerable length of time without the slightest benefit, and it was feared the disease would soon terminate in glanders, and my advice was sought.

Symptoms.-Pulse and respiration normal; no cough; copious discharge-muco-purulent-from the nose, which adheres to the alæ nasi ; the membrane of the nose is highly vascular; the submaxillary glands are enlarged, and tender to the touch; the animal 
is rather low in flesh and not in a thriving state; appetite pretty good; fæces and urine normal; the hair is dry and harsh when felt, and the skin adherent.

Treatment.-To have Merc. v., 20 drops, sixth dilution, three times a day.

10th.-Greatly improved in every respect. Continue medicine. 20th.--Since last report he has had the medicine as before, and is now quite well.

\section{Case III.}

A horse belonging to A. Birley, Esq., Didsbury, near Manchester. This horse is 16 years old, 15 bands 3 inches high, used for carriage purposes; and although delicate when joung, has had good health during the last ten years.

Uctober 14th, 1859._- Present state: Pulse and respiration slightly quickened; discharge of thick yellow matter from right nostril; accumulation of greenish pus on the nasal alæ; horrible foetor; the box being ill-ventilated, the stench is intolerable and sickening; the right sub-maxillary gland is swollen and tender; appetite good.

Admitted to the hospital on the 17 th.

Treatment.-Kali Bichrom. 1, 20 drops night and morning; the nostrils to be fumigated with the same drug. The discharge began to decrease from the fourth day, and the horse was discharged cured on November 22d.

\section{4.-Collections of Pus in the Nose.}

\section{IN HORSES.}

In horses, after catarrh, matter is apt to accumulate in the frontal and nasal sinuses. There is, in addition to discharge of matter from the nostrils, swelling of the glands under the jaw, and dull sound when the bone of the face over these sinuses is struck with the knuckle. When only one side is affected, the difference between the hollow sound of the healthy side and the dull sound 


\section{$15 \frac{1}{ \pm}$ DISEASES OF THE RESPIRATORY ORGANS.}

of the diseused one, is sufficiently marked to decide as to the exact locality of the collection. In some cases, that side of the face is also swollen, from the upward pressure of the imprisoned matter beneath.

\section{Treatment.}

Here it is imperative to remove a circular piece of bone by means of the trephine, and to remove the matter by thoroughly syringing out the cavities with warm water. Then, three times a day inject Hydrastis Lotion, * and give 20 drops of Hydrastis three times a day. Mercurius, Kali Bichromicum, and Arsenicum are of service in such cases, in the same doses, as also Carbolic Acid, 10 drops three times a day.

\section{5.-Collection of Matter in Guttural Pouches. IN HORSES.}

In cases of catarrh where the disease has extended backwards, as well as in strangles, the guttural pouches are often involved in the inflammation, and the result may be that pus collects. When both sides are affected, the enlargement may be so considerable as to interfere with breathing, and threaten suffocation. The discharge may escape into the throat, or make its way through the skin at the angle of the jaw. When it flows into the nose, we find the discharge issuing from one side when the horse holds his head down, as whilst grazing, because this position enables the matter to flow out of the pouch. Usually, the discharge does not escape regularly from

\footnotetext{
* See list of local applications.
} 
day to day ; it is much increased during work, and by the dependent position. The swelling may cause roughness of breathing, or roaring, and, when large, causes the neck to be thick.

\section{Treatment.}

In chronic cases of this kind, turning the animal out to grass-in other words, compelling him to keep his head for a considerable time in a position calculated to allow the matter to escape-is often a successful measure. In others, an operation is necessary.

\section{6.-General Remarks on Nasal Discharges. IN HORSES, \&c.}

In the preceding pages of this chapter it has been shown that discharge from the nose is one of the most prominent symptoms of coryza, of nasal gleet, and of disease of the nasal sinuses, and guttural pouches.

It is, in addition, observed under the following circumstances:-

1. When, as is stated at page 34 , a diseased tooth sets up irritation and inflammation in the upper jaw, the result being the formation of matter which finds its way into the nose, and thence is discharged through the nostril. The discharge is confined to one side, is very offensive, and sometimes bloody.

2. When the bones entering into the formation of the nose (nasal, turbinated, upper jaw, \&c.) are diseased, in consequence of the intrusion of foreign bodies. In disease of the turbinated bones, acempanied by collection of pus, the face swells, and there is obvious deformity. 
3. In glanders. This disease will be found fully discussed in another part of this work. Many of the diseased conditions already described have, from the nose symptoms, been mistaken for glanders, and many valuable animals have been destroyed in consequence. In a doubtful case, inoculate an ass with the nasal discharge; if glanders follows, all doubt is removed.

\section{General Treatment.}

This comprises the removal of diseased teeth, foreign bodies, and rotten bone. Collections of matter must be removed, either by injections into the nose, or by irritating fumigations to compel sneezing, or by position, or by operation.

In diseases of the nose such as I have already described, I consider Hydrastis Canadensis, injected in solution, or inhaled by steaming, one of the very best local applications that can be used.

For internal use, I place, in the order of their value, Kali Bichromicum, Hydrastis, Mercurius, and Arsenicum.

Doses. - 20 drops for horses and cows; 10 for sheep and pigs.

Carbolic Acid, in doses of 10 drops, is a good remedy.

\section{7.-Bots in Sheep.}

Bracy Clark, in his memoir on Bots, states that the eggs of the sheep bot are deposited on the margin of the nostril. He proceeds - "The moment the fly touches this part of the sheep, they shake their heads and strike the ground violently with their fore-feet, at the same time running away and holding their noses close to the ground, and looking around them on every side to see if 
the fly pursues, and as they go along they often smell also to the grass and look anxiously into it, lest one should be lying in wait for them; if they observe one, they gallop back again, or take some other direction."

The larvæ are found in the maxillary and frontal sinuses. When fully developed, they drop out through the nostrils, remain on the ground for about two months in the pupa state, and then take to wing.

\section{Treatment.}

For the parasites in the nose of the sheep, nothing can be done except to cause these animals to breathe the fumes of burning Sulphur, or Tobacco.

\section{8.-Influenza.}

\section{IN HORSES.}

This disease is more definitely called Catarrhat Fever, and Epizootic Catarrh. When pure, uncomplicated, and typical, it consists of two factors - a general fever, and a specific affection of the mucous membrane of the nose, eyes, mouth, throat, and air-passages.

It is supposed to arise from some peculiar atmospheric condition - nobody knows what ; it prevails extensively in certain years, striking a certain number of animals at one and the same time; it is most common in spring and autumn, and affects young horses more frequently than old.

The likeness between it and human influenza is singularly close. In an ordinary case of the genuine-disease, the first symptoms are those of febrile excitement. The animal is dull, listless, off his feed. The pulse is 
quickened and feeble, the breathing slightly hurried, the urine scanty and high-colored, the bowels costive, the skin of variable temperature, the mouth dry and hot, the membrane of the nose and eyes reddened. At this period, there is a congestive swelling and dryness of the mucous membrane of the nose, frontal sinuses and eye - a condition which subsequently extends along the air tract. Presently, the affected membrane gives forth a secretion, which is at first thin and irritating, afterwards thicker, tougher, and mattery. At this period, the eyelids are swollen and tears flow down the face; and there is a discharge from the nose, attended with sneezing, and also from the mouth. In addition, we find indications of sore throat, pain and difficulty of swallowing, quidding of food, external swelling of the throat at the angle of the jaws and in the space under and between the jaw bones, frequent, irritating cough, and greater acceleration of the breathing. It is a peculiarity of influenza that throughout its whole course the nervous system is profoundly affected; as witness, the early depression of spirits, languor, muscular weakness, want of energy, disinclination to move, staggering gait, halting and dragging of the hind legs, and stupor, or (in some cases) a tendency to excitement.

In a few days, more or less, these symptoms begin to subside and finally disappear, leaving the patient, however, weak and out of condition for a little while longer, according to the mildness or severity of the prevailing epizootic.

In practice, influenza is not often found thus typically well-marked and clearly defined as a catarrhal fever. The attacks of one year differ much from those of 
another, and even at the same period individual cases present great diversity of symptoms. For instance, in one case the fever may be of a somewhat decidedly inflammatory character; in another, it may be low and malig. nant. The fever may predominate over the catarrh, or the catarrh over the fever. Again, serious complications in contiguous, continuous, or distant organs may arise during the course of influenza, without the latter ever losing its specific features. I may instance inflammation of the lungs, pleura, heart, \&c.; and more particularly disease of the digestive mucous membrane. These different phenomena of a concrete disease have been classed by Hering and other continental writers into three groups, somewhat arbitrary to be sure, but yet sufficiently inclusive as to be free from grave objections. They are: 1st, The catarrho-rheumatic ; $2 \mathrm{~d}$, The gastro-erysipelatous, where there are the swellings of cedema; and $3 \mathrm{~d}$, The gastric, or bilious-rheumatic.

In this country, the different varieties of influenza that have occurred within historical times, may practically be arranged into two grand classes: 1st, That whose symptoms are above narrated, in which the respiratory mucous membrane, and respiratory organs secondarily, are the chief local seats of inflammation: and $2 \mathrm{~d}$, That in which these organs are less affected than the mucous membrane of the digestive canal, and the digestive organs.

In this last variety, the catarrhal symptoms referrible to the nose, eyes, throat, and lungs, are but slightly marked - the force of the disease is spent elsewhere. We observe loss of appetite; thirst; symptoms that may, without stretching a point unduly, be interpreted as indi- 
cating headache and nausea; coated tongue and accumulation of soapy saliva in the mouth; a yellow color of the membrane of the eye, nose, and mouth, and likewise of the skin ; slimy evacuations. In some years, as in the spring of 1865 , these symptoms have been especially prominent. In some cases the eyelids were everted, the legs much swollen. Symptoms of headache - resting the head on the manger, or hanging it low down almost to the ground. There were often rheumatic pains in the legs, known by the animal being lame.

\section{Treatment.}

The patient should be placed in a clean, airy, wellventilated, loose box, be sufficiently clad, and fed on oatmeal and linseed gruels, and bran mashes.

The most useful medicines are the following:

Aconite is seldom required at the onset, except in those comparatively rare instances of the disease when the attendant fever is of a decidedly inflammatory character. It may, however, be called for during the progress of the disease, when local congestions and inflammations arise.

Belladonna is indicated by swollen, closed eyes; flow of tears ; sore throat; pain and difficulty in swallowing; tenderness and swelling of the glands about the neck and jaw ; irritating cough ; indications of headache or of delirium.

Ammonium Causticum is a valuable remedy for the complication of congested lungs, which may be known by hurried, labored breathing, dilated nostrils, cold skin, \&c.

Mercurius is indicated by similar catarrhal symptoms as those which demand Belladonna, and in some cases 
these two medicines may be used in turn with great benefit. Mercurius is likewise valuable for foul tongue ; discharge of saliva; offensive breath; yellowness of the eyes, mouth, and skin; slimy, bilious evacuations.

Nux Vomica is peculiarly called for when the bowels are constipated, or relaxed at one time and confined at another; the evacuations hard, lumpy, and covered with mucus; tendency to drowsiness and apparent paralysis of the hind legs.

Phosphorus is required when inflammation of the lungs is at hand or present, which may be known by the symtoms detailed in this chapter on that disease.

Bryonia must be given when any rheumatic or pleuritic element exists - short, grunting breathing; pain to the touch in the intercostal spaces; friction sounds heard on applying the ear to the affected side, etc.

Arsenicum is required for great prostration of strength and spirits; feeble pulse; and in short for the obvious low symptoms which characterize the worst variety of this disease - that in which the power of life is profoundly and rapidly depressed.

Dose.-20 drops of any of the above medicines; repeat each dose every one, two, three, or four hours according to the necessities of each case, and the resulting improvement.

In this disease, in 1872, I used Carbolic Acid, ten drops, three times a day, in a little water, with good success.

\section{1.-Strangles.}

IN HORSES.

Strangles is the common name for a disease compounded of fever, and a specific phlegmonous inflamma- 
tion of the arcolar tissue about the throat, and of the glands under the jaw.

It is most common between the fourth and fifth year; in cold, damp springs and autumns; and attacks almost every horse.

It begins with loss of appetite, languor, occasional cough, feverishness, staring coat, \&c., followed by sore throat, pain in swallowing, and swelling and tenderness about the throat externally. This swelling is at first hard, then it softens and breaks, unless opened by the lancet, and discharges matter. Usually, the disease now abates, and recovery is rapid. Sometimes, however, abscesses appear in other parts of the body-the back, shoulders, legs, breast, \&c. This is Irregular StraxGLES. Inflammation of the windpipe, or of the lungs, sometimes follows strangles.

\section{Treatment.}

Foment the throat three times a day, and afterwards put on a hot bran poultice.

As a rule, the only medicines required in simple strangles are Belladonna and Mercurius, 20 drop doses, every four hours, turn about. In order to hasten suppuration, Hepar Sulphuris is useful, given in the same doses and time. Arsenicum may be necessary when strength and appetite are not quickly recovered.

\section{2.-Congestion of the Lungs.}

\section{IN HORSES, \&c.}

The sudden and severe engorgement of the lungs with blood, so often met with in horses, is most frequently 
caused by a long run in hunting, or by any other severe and long-continued exertion. It also arises when a horse has been over-fatigued by a hard day's work, especially if he has been exposed to damp and cold. Under such circumstances this disease is very common amongst the horses of our large brewers, and railway and carrying companies. By the adoption of a more rational system of treatment, than that of bleeding, blistering, and so on, many hundreds of pounds would be annually saved.

The symptoms are beyond mistake. The patient's pulse is strong and full, and may count up to 100 per minute. The heart, on placing the ear to the side, is heard beating with unnatural agitation. The breathing is oppressed, labored, panting, and may count 50 or 60 . The nostrils are widely dilated and work heavily. The eyes are staring, and anxiety and distress are written in the expression of the countenance. Sometimes blood flows from the nose in small quantity, or, if a ressel be ruptured, fatal bleeding may take place suddenly. The legs and ears are cold. These symptoms may end in death, or they may run on to the development of pneumonia, or bronchitis, or pleuro-pneumonia.

\section{Treatment.}

In simple congestion of the lungs, I find the best remedy to be Ammonium Causticum. The following is a case in point:-

On September 19th, 1857, I was requested to visit a valuable horse, the property of Mr. Walter Carter, of Manchester.He had recently come into the owner's possession, had been put to severe work, to which he had hitherto been unaccustomed, and on the 18th was permitted to drink copionsly of cold water, whilst perspiring and exhausted, after a hard day's labor. Shortly after- 
wards he had a rigor so violent that his legs tottered under him. Three hours after this shivering, I found the following symptoms: Pulse strong, full, and 100 per minute; respiration labored, heaving, and 84 per minute; conjunctiva injected; eyes watery; mouth hot, and clammy to the touch; corrugations of the cutaneous muscle along the side and shoulder; general surface warm, the extremities cold; the nasal membrane preternaturally vascular, \&c.

To have 20 drops of Ammon. Caust. 1, in a wine-glassful of water, every hour.

On the following morning the pulse counted 28 in the minute, and intermitted occasionally; all the other symptoms had disappeared, and he ate, drank, dunged, and staled as if nothing had been amiss. At two o'clock of the same day the pulse had risen to the healthy standard, and had assumed the usual character;--in short, the horse was all right, and resumed work next morning.

\section{3.-Bronchitis-Inflammation of the Bronchial Tubes. \\ IN HORSES.}

This is a very common disease, in its acute form, amongst all domestic animals, especially the horse. It may come on and run its course without any preceding or accompanying disorder; very often it succeeds sore throat, catarrh, and congestion of the lungs. In a considerable proportion of cases, there is more than pure bronchitis - a touch of pure pneumonia, constituting Broncho-Pneumonia.

Bronchitis arises from exposure to east winds, damp and cold, changes of the weather, \&c.

The symptoms first observed, in sudden and uncomplicated bronchitis, are languor, loss of appetite, thirst, ears and legs cold, pulse full and quick, and breathing accelerated. The cough is at first slight, and afterwards 
frequent and painful. The in-taking act of breathing is attended with noise, from the diameter of the tubes being diminished by a swollen condition of the mucous membrane lining them. This sound is heard best at the front of the chest, and also on applying the ears to the side. At a subsequent period, an increase of mucous secretion takes place, at first frothy, afterwards thick and mattery; it both runs from the nose and is coughed up. At this time, loud rattling can be heard in the chest on applying the ear to the side. The symptoms now begin to decline, and recovery soon takes place.

\section{IN OTHER ANIMALS.}

All our domestic animals, equally with the horse, suffer from bronchitis. The causes and symptoms are the same as those above described.

\section{Treatment.}

Aconite should be given for hot, dry mouth; quick, full pulse; thirst, and the other symptoms of feverishness.

Bryonia is indicated for quick, difficult breathing; rattling in the windpipe and in the air-tubes in the lungs ; frequent, dry, irritating cough; discharge of thick phlegm.

In the majority of cases, at the beginning, it will be necessary to give these two medicines alternately.

Belladonna is required for soreness of the throat; pain and difficulty of swallowing; violent fits of coughing, \&c.

Antimonium Tartaricum is indicated when the discharge of mucus is very copious; the cough loose; the rattling loud; and the breathing much distressed. 
Kali Bichromicum is indicated when the phlegm is tough, sticky and stringy; in old-standing cases; and when the tongue is covered with yellow fur.

Sulphur, I generally give when all the violent symptoms are on the decline, with a view to hasten recovery.

Doses._-20 drops for horses and oxen; 10 for sheep and pigs.Give each dose every one, two, or three hours, according to the violence of the symptoms.

\section{Case of Acute Bronchitis.}

On March 5th, 1867, a horse belonging to Messrs. Mowlem, Burt, \& Freeman, had the following symptoms:-Pulse 104 and soft ; respiration 48 and labored; eyes and nose red; loud rattling in airtubes; no appetite; fæces lumpy and coated with mucus. To have Aconite and Bryonia, 20 drops every hour, for five hours, then every two hours. On the 6 th-pulse 66 ; respiration 36 ; eats a little; rattling not so loud; continue medicines. On the 7 th - pulse 60 ; respiration 25; frequent cough and discharge of mucus; rattling much less; looks better generally; continue medicines. On the 9 th-pulse 48 ; respiration 14 ; in other respects doing well ; continue medicines. On the 11th-well; to have 20 drops of Sulphur, night and morning, for two or three days.

\section{Case of Chronic Bronchitis.*}

On the 25th of December, 1860, I visited a horse, the property of Messrs. Sewell. He was ill three months ago, under allopathic treatment, and has had a copious discharge from the nose ever since.

Symptoms. - Violent rattling in the bronchial tubes; copious discharge of frothy mucus from both nostrils ; pulse 48 and full ; respiration 16 per minute; frequent, moist cough.

Treatment.-To bave Ant. Tart. $10 / 2$, night and morning..

This treatment was continued for eight days, when the horse was quite well. Ten months have now elapsed, and the horse has been at work ever since, with the exception of a few days, when he was laid up from a severe injury.

*Quoted from "Practical Reply to Sir B. Brodie's Letter." 


\section{4.-Pneumonia-Inflammation of the Lungs. \\ IN HORSES.}

Inflammation of the proper substance of the lungs is rare, in comparison with a compound disease in which the air-tubes, or the pleura covering the lung, is more or less inflamed at the same time. When the air-tubes and lungs are inflamed, we call the disease Broncho PNEUMONIA, and we find the joint symptoms of the two distinct diseases more or less mixed up together in the same case. When the pleura and lungs are affected, the disease is called Pleuro-Pneumonia, which presents the combined symptoms of the two individual affections.Nor is it rare to find symptoms of bronchitis, of pneumonia, of pleurisy, and even of heart disease, co-existing in the same patient.

Fat, full-blooded animals, and those that are overworked or severely galloped are predisposed to this disease, and exposure to cold and damp, and to variations of temperature, determine the attack.

In some cases, the disease begins with a more or less violent fit of shivering, the ears and legs being cold, the skin staring, the nose pale, and the animal languid and depressed. In others, slight cold, want of appetite, cough, \&c., are first observed. In either case, febrile reaction comes on ; the pulse is frequent and full, counting 60 or 70 in the minute; the breathing is short, labored, and 30 or 40 per minute, or even much higher; the membranes of the nose and eyes are reddened; the mouth hot and dry; the expression of the countenance anxious and distressed; the bowels costive, and the urine scanty and high colored. 
At a somewhat later period, the breathing becomes more labored and heaving at the flanks; the nostrils are widened and in full play; the neck is stretched out at full length; the nose and head poked forward; the animal stands fixed in one place, with his legs separated from each other-in a word, he instinctively postures himself in such a manner as to bring the "extraordinary muscles" of respiration into full action. At the same time, the membranes of the nose, eyes and lips have a dark bluish tinge; the legs and ears are remarkably cold, whilst the skin elsewhere may be moderately warm; patches of sweat break out here and there; the cough is only occasionally heard, or it is frequent, hard, painful, and attended with the discharge of reddish colored mucus.

Still later, the pulse is small, weak, and can hardly be counted; the breathing is still more labored and difficult; the expired air hotter than usual; the mouth cold and clammy; the teeth are ground; the muscles twitch and quiver; the eyes are dim, heavy, and glassy ; the animal very weak and drowsy; he wanders unconsciously around the box, or leans against the manger; he soon staggers and falls down; and whilst attempting without success to get up again, he groans, struggles briefly, and dies.

The physical signs leave no doubt as to the nature, severity, extent, and complications of the disease; and give valuable information as to the probability of recovery. One or both lungs may be involved. In the diseased parts of the lungs a sound is heard, resembling that which is produced when one's hair is rubbed between the finger and thumb, close to the ear. This 
sound is learnedly called "crepitation;" it denotes the first stage of pneumonia, when the lungs are engorged with blood or bloody serum. In the same parts the natural healthy sound is obscured, and as the disease advances, displaced by the morbid one. As compared with the healthy lung, the diseased part gives out a dull sound when tapped, as is done when the human chest is "sounded." In the second stage, the lung loses its spongy structure, and becomes dense and solid. Neither crepitation nor the natural sound can now be heard, but instead, a blowing sound proceeding from the larger bronchial tubes, which are surrounded by the solid lung. At a still more advanced stage, the sounds are rattling, from the passage to and fro of air through the effused fluids, or products of inflammation. These either cease, and are gradually replaced by the gentle, breezy murmur of health, or continue, and then indicate suppuration of the lung.

\section{IN OXEN AND SHEEP.}

In these animals, pure pneumonia is rarer than in others. When it does occur, the generic symptoms are analagous to those observed in the horse, as above detailed.

\section{Treatment.}

Place the animal in a well-ventilated box, and let plenty of air in. In the horse, put on warm clothing; hand-rub and bandage the legs; and give small quantities frequently of whatever food he will eat.

The following are the best remedies:-

Ammonium Causticum in those cases which begin with languor ; coldness of the legs, ears, and nose ; rough, star- 
ing coat; quickened, difficult breathing; pulse small, weak, and frequent.

Aconite is indicated when febrile reaction comes onthe pulse being quickened and full; the breathing labored and panting; the mouth hot and dry; the membranes of the eye and nose injected.

Bryonia is especially required, often in alternation with Aconite, when there are symptoms of bronchitis, such as loud rattling from the first in the air-tube, heard when the ear is applied to the breast or side; the cough loose and attended with discharge of frothy phlegm, \&c.

Phosphorus is of the greatest value in this disease, especially in the second stage, when the lung is solidifieda condition which can be ascertained by detecting special physical signs. Also, when the breathing is very labored and distressed; and when a reddish or yellowish discharge is coughed up.

Arsenicum is often of service in extreme cases, when there are great depression; weak pulse; cold, clammy mouth ; purging; no appetite.

Sulphuir I always give as soon as the acute symptoms have yielded.

D os Es.-20 drops for horses and oxen; 10 for sheep and pigs.Give a dose every one, two, or three hours, according to the violence of the symptoms.

The following case, quoted from "Practical Reply to Sir B. Brodie's Letter," illustrates the symptoms and treatment of an ordinary case of pneumonia in the horse :-

On the 13th of August, 1860, a horse, the property of the Midland Railway Company, was brought to my establishment, having been observed to be unwell for two days previously. The hostler 
unfortunately, gave a dose of aloes, which, however, did not purge; but it affected the kidneys and nervous system, for the horse was weak in his back, and swayed from side to side, and the left hind leg was partially paralysed. These effects I have frequently seen, when aloes has been given, and especially after the use of Cupiss' balls.

Symptoms.-Pulse 72 per minute; respiration 36 per minute; copious crepitation throughout the whole of the left lung, particularly in the upper portion, or the site of the saddle; no friction sound anywhere; frequent, short, hard, and suppressed cough, belly tucked up, and marked with deep indentations along the margin of the ribs; the conjunctival and nasal membranes vascular; nostrils expanded; urine scanty, and high colored; appetite bad; the animal dull, and hangs his head.

To have Aconite, 20 drops, first dilution, every three hours, in a wine-glassful of water.

14th.-Pulse 64 per minute; otherwise the same. Continue Aconite as before.

15th.-Pulse 56, and respiration 21 per minute: appetite improved; the animal is more lively; the rattles in the inferior and middle third of lung nearly gone; copious still in upper third.

To have Phosphorus, 20 drops, third dilution, every three hours.

16th.-Considerable amendment; pulse 56, and the respiration 16 per minute; is lively, and appetite good; rattles nearly gone, except a few along site of saddle; does not cough so much; conjunctival and nasal nembranes less injected; laid down last night, -horses never lie down while they are ill with inflamed lungs, and it is always a good sign of returning health when they do so,otherwise improving. Continue the medicine as before.

17th.-Improving; pulse 46, and respiration 12 per minute; the rattles are nearly gone.

To have Sulphur, 20 drops, sixth dilution, night and morning.

19th.--Pulse 42, and respiration 10 per minute; was put into the yard to water, and began to play. Continue medicine.

2uth. -Well. 


\section{5.-Pleurisy-Inflammation of the Pleura. IN HORSES.}

This disease, which consists of inflammation of the serous membrane lining the cavity of the chest and covering the lungs, attacks the horse more frequently than any other animal. As I have already stated, it often coexists with pneumonia. Exposure to cold, and injuries of the chest, broken ribs, \&c., are the most frequent causes.

It usually begins with shivering, followed by dry mouth, languor, anxious expression, \&c. The pulse is quickened, hard, and wiry. The respiration is characteristic, the chest being comparatively still, whilst the muscles of the abdomen are in full play, and whereas the act of expelling air from the lungs is easy and prolonged, that of inspiring air is short and constrained.The cough is short, hacking, and greatly increases the pain in the side, or "stitch" which the horse feels. The horse stands still in one position, in a crouching attitude, with neck stretched out and his head protruded; he seems uneasy and in pain, and afraid to move. On applying pressure to the affected side, he flinches and grunts. The ear placed on the side hears a rubbing friction sound. The skin over the inflamed chest is thrown into folds, and the flanks are tucked up; the skin around the openings of the nostrils and around the mouth is wrinkled. When effusions of serous fluid occupy the cavity of the chest-Hydrothorax-the breathing becomes more labored and quicker, but less grunting; the pulse feebler; the friction sound diminishes, or ceases; dullness on percussion is detected at 
the lowest part of the chest; and, in unfavorable cases, drowsiness, prostration of strength, and cold sweats usher in death.

In a large proportion of cases, the membrane covering the heart, and in a few, even the heart itself, is involved in inflammation at the same time as the pleura.

\section{IN OXEN, SHEEP, \&C.}

These animals, and likewise the hog, may be attacked with pleurisy, from the same causes as the horse, the most frequent being one out of the many circumstances summed up in the expression, "exposure to cold."

The symptoms, especially the peculiar character of the breathing, are identical with those of the same disease in the horse.

\section{Treatment.}

Aconite is required in the early stage, when the pulse is hard and full, the breathing quickened, the mouth hot and dry, etc.

Bryonia is required, in alternation with the last medicine, when the breathing is short and catching; the sides painful to the touch; the cough short and restrained; and when the animal grunts when the side is pressed against.

Sulphur is useful when all the severe symptoms are on the decline.

Doses._-20 drops for horses and oxen; 10 for sheep and pigs.

For the treatment of Dropsy of the Chest, so apt to supervene upon pleurisy, refer to the remarks on that subject. Also consult the observations upon "Pneumonia," and "Bronchitis." 
The two following cases are illustrations of pleurisy as it occurs in the horse :

\section{CASE I.}

On M ty 12th, 1802, Mitchell Henry, Esq., of Harley street requested my attendance on one of his carriage-horses. It was in the breaker's hands, and was supposed to be suffering from sore throat, for which mustard was applied. I found the following symptoms in chief: Pulse 60 ; respiration 36 and short; a grunt at every expiration; intense pain in the side when pressure was applied; friction sounds ; frequent suppressed cough ; membranes of eyes and nose injected. To bave Aconite every 3 hours. On the 13 th - pulse 48 ; respiration 24 . On the 14 th - pulse 44 ; respiration 16; side less painful; in other respects better. To have Aconite and Bryonia alternately. On the 17 th - the animal was convalescent - to have Sulphur night and morning for a few days.

\section{CASE II.}

On November 20th, 1861, I attended a horse belonging to Dr. Davison. It had been hard worked in rough, stormy weather. The symptoms were:- pulse 40; peculiar jerking action of the heart, felt at the left side and heard; pain on pressure between the ribs on the right side, causing grunting; single, suppressed, dry cough, which evidently causes pain; animal very feeble when made to walk. To have 20 drops of Bryonia 1, every three hours. On the 21st, much the same, only the side not so painful. To have Digitalis alternately with Bryonia every two hours. On the 22d, much better; pulse 32 ; heart not so jerking; appetite better; no cough and no pain in side. Continue medicines night and morning. On the 25th, well.

Still further to illustrate different forms of lung diseases, as well as their treatment, I cannot do better than reprint the following cases from the British Journal of Homœopathy, for 1858 : 


\section{Case I.-Panemonia (Double) and Pleurisy.}

On November 15th, 1859, I visited a splendid carriage-horse belonging to Thomas Brocklehurst, Esq., the Fence, Macclesfield. This horse is 4 years old, 16 hands high, and was bought in the metropolis a few weeks ago. He has been quite well until a few days since, when, after ordinary carriage work, he was observed to cough, and to be otherwise out of sorts. The coachman thinking that it was a case of simple sore throat, gave a few doses of Belladonna and Hepar Sulph.; but as he continued to get worse, I was summoned by telegram. Two or three doses of Aconite were given before my arrival.

I found the following symptoms: Pulse 64, soft, and compressible; respiration 36, and slightly embarrassed; eough frequent, hard, and single; conjunctiva injected and yellowish; nasal membrane also increased in vascularity ; mouth hot and slimy ; appetite bad; pain and grunting on intercostal pressure; nether lip pendulous; distinct crepitation in superior half of left lung; some mucus rales in the middle; loud friction sound along the middle and infero-posterior portion of right lung, with crepitation higher up, etc.

Treatment.-To have Aconite and Bryonia 1, 20 drops every three hours alternately.

16th.-Much the same. To have Phosphorus and Bryonia 1, 20 drops every two hours alternately.

17th.-Pulse 70 ; respiration 30 ; grunting and catching during respiratory acts; suppressed, short cough; has eaten mashes and some hay; left lung sounds rather better; those of right unaltered; mucous membranes less injected.

Continue medicines.

18th.-Pulse 58; respiration 24 ; occasional grunts; the head is held higher; the freces are soft; the urine less turbid; the appetite improved; the horse is more lively; bronchial respiration along middle of right lung, with small crepitation above and below; cough still frequent.

Continue same medicines every three hours alternately.

19th.-Pulse 46 : respiration 18 ; only a few subcrepitant rales here and there in left lung; instead of the tubular breathing of 
yesterday, there is now the returning crepitation of health, mingled with the respiratory murmur; healthy sound in other parts of this (right) lung; friction gone; cough loose; some stringy expectoration; has been resting several times yesterday and to-day. Continue medicines every four hours.

21 st.-Pulse 37 ; respiration 14 ; limited rales here and there in both lungs, especially in superior part of left : in all other parts the respiratory murmur is re-established; much improved in all other respects.

Continue medicines.

23 d.-Pulse 32 ; respiration 14 ; all abnormal sounds are gone; appetite good.

To have Sulphur 6, 20 drops three times daily.

26th.-All right.

\section{Case II.-Pleuro-Pneumonia in Horse.}

On January 20th, 1858, Messrs. Syddall Brothers, calico printers, Chadkirk, near Manchester, requested my attendance on their mare, which had been coughing for several days previously.

Her symptoms are these: Pulse full, strong, and 70 per minute; breathing labored, difficult, and 40 ; cold legs, nose, and ears ; frequent, short cough ; no appetite; crepitation through whole of left lung.

To have 20 drops of Aconite 1, erery three hours.

On the 21 st, the pulse is down to 58 , and the breathing to 36 per minute; the appetite is better; in all other respects improved.

To have Aconite 1, and Phosphorus 3, in 20-drop doses, every three hours alternately.

On the $22 \mathrm{~d}$, the pulse is 44 , and the breathing 12 per minute; all the crepitation is gone; the appetite is good; has laid down.

Continue same medicine every six hours.

On the 24th, she is much the same.

To have 20 drops of Arsenicum 1, every six hours.

On the 26th, she is much worse, in consequence of having been most injudiciously walked out this morning from a warm stable into a frosty and piercing cold air. The pleura is now inflamed, 
the symptoms being short, suppressed cough ; pain in the intercosal s races ; grunting, etc.; no friction can be detected.

To have 20 drops of Aconite and of Bryonia, each of the 1st dilution, every three hours alternately.

On the 28th, the pulse is 48 and weak; breathing less frequent, and not so difficult and painful; looks lively, appetite good.

To have Arsenicum and Phosphorus, 20 drops of the 1st dilution, every three hours alternately.

On the 30th, all abnormal sounds in lungs gone; eats well; rests as in health, \&c.

To have Arsenicum as before, three times a day.

February 1st.-Well.

\section{Case III.--Typhoid Pneumonia.}

On February 23d, 1860, Messrs. W--, the extensive London carriers, placed an aged mare under my treatment. She has been treated allopathically for seven or tight wetks, and is prononnced incurable. On this date the symptoms are:- Pulse 73, weak and small; respiration 36 , quick and short; the cough is painful, carernous, and frequent; discharge from both liostrils is copious, muco-purulent, and brownish; considerable expectoration from the mouth; the breath, sputum, and nasal discharge are horribly foetid, especially after a paroxysm of coughing; when the animal coughs, the head is held down to the ground, and the flanks beave; the eyes are dull and heavy; the appetite greatly impaired; the urine scanty; the bowels costive and the evacuations offensive; the extremities are cold; the mare is exceedingly weak and in very low condition. On examining the lungs, 1 found $t$ ubular breathing in inferior third of left side, loud rattles in middle third, and subcrepitation in upper third; there were scme scanty, indistinct rattles throughout right lung.

Treatment. - To have 20 drops of Phosphorus 1, three times a day. 24th.-Pulse 60 ; respiration 24 ; has eaten more to-day than she has done since the disease began. Continue medicine.

25th.-Appetite still improving; the hair is more glossy; returning crepitation in hepatized portion of left lung; respiratery murmur returning in inferiur portion. Continue medicine.

27 th. - Pulse 52 and stronger; eyes brighter; cough less fre- 
quent; profuse muco-purulent expectoration; o lor of breath less offensive; healthy breathing gradually returning in both lungs. Continue medicine.

March 3d. -Steady general improvement since last report ; still a few rattles in inferior portion of left lung. To have Sulphur 6, 20 drops three times a day.

10th. - Went to work with the team.

Remarks.-A more satisfactory case than this it is impossible to cite; a more conclusive proof of the efficacy of Phosphomus in advanced stages of Pneumonia it is impo:sible to furnish. I have frequently opened horses that have died under allopathic treatment of this disease, with exactly the same symptoms as in the above case, and have always found one or more abscesses in the lungs.

\section{Case IV.-Pleuro-Pneumonia.}

On April 7th, 1852, a cart-horse belonging to Messrs. Molyneux, Webb \& Co., the eminent glass manufacturers of Mauchester, stood, for a considerable time, exposed to rain and col: and was shortly afterwards observed to be unwell.

There are the following symptoms:-Pulse full, hard, and 68 per minute; breathing 32 per minute ; short, suppressed inspiration; long, slow expiration, attended with a wheezing grunt and catching during breathing; pain when the intercostal spaces are pressed against, with elicitation of characteristic grunting sound; the skin over the affected side is thrown into folds, in consequence of contraction of the cutaneous muscle; the skin about the sides of the nostrils, and at the angles of the mouth is also wrinkled; the tongue is covered with a yellow fur; the bowels are constipated ; there is a frequent, short, suppressed cough, evidently attended with pain; bronchial rattles are distinctly audible in the inferior third of the right lung, whilst in the middle third, small crepita tion can be detected.

To have Aconite and Bryonia of the 1st dilution, 20 drops of each every two hours alternately.

On the 8 th. - The pulse is 64 , and the respiration 24 per minute; no abnormal sounds in the right lung, but in the middle portion of 
the left there is copious crepitation, and friction sounds, which latter are more marked when the animal coughs.

To have three doses of Bryonia 1, at intervals of two hours ; and then the same dose of Phosphorus 3, every three hours.

On the 9 th. - The pulse is 52, and the respiration 26 per minute; the cough is less frequent and less contrained; the friction sounds are gone; the crepitation is less marked; the appetite is improved; the dung and urine are natural; the horse looks more lively.

Continue the Phosphorus as before.

On the 10th.- The pulse is 48 , and the respiration 22 per minute; better in all other respects; healthy vesicular breathing is resumed in the inferior half of the lung; scanty crepitation in posterior portion of superior half.

To have 20 drops of tincture of Sulphur, every four hours.

On the 12th. - The pulse is 44, and the breathing 16 per minute; all abnormal sounds have disappeared; improving otherwise.

Continue same medicine.

On the 14th.-Considerable amendment. A few more doses of Sulphur will restore my patient.

\section{Case V.-Broncho-Pneumonia in Horse.}

On May 6th, 1852, a horse belonging to Mr. McCaldon, horse dealer, of Manchester, presented the following symptoms:-Pulse soft, full, and 60 per minute; breathing difficult, 80 per minute and attended with loud mucous rattles at the bifurcation of the trachea; violent shivering ; frequent, loose cough ; anxious countenance; ears, legs, nose, etc., very cold; pituitary membrane dry, and of a bluish color; conjunctiva intensely vascular: throughout the whole of both lungs there are loud, bronchial, mucous rattles. which mask every other sound.

To have 4 drops of Ammonium Causticum, every hour.

On examin ttion three hours afterwards, the pulse is full and counts 100 per minute; the breathing 68 , and attended with less audible rattles; the entire surface burns with heat.

To have 10 drops of tincture of Aconite, every hour.

On the morning of the $7 \mathrm{th}$, the pulse is 60 , and the breathing 46 
per minute; there are fewer rattles in the larger bronchi heard at the breast.

To have 20 drops of Aconite 1, every four hours.

In the evening of the same day, the pulse is 60 and rery.weak, the breathing 24 and much less difficult; there is great debility and no appetite.

To have three doses, 20 drops each, of Arsenicum 1, at intervals of two hours; then resume the former medicine.

From the 8 th to 10th.-The Aconite has been continued in the same way. The pulse and respiration are becoming more natural, and the general appearance indicates recovery.

On the 11th.-'I'he pulse is risen to 49 , and the horse is altogether worse. On examining the lungs, which I bad not done since the first day, in consequence of the favorable progress of the case, 1 found, along the inferior third of left lung, dullness on percussion, absence of respiratory murmur, kronchial respiration, and considerable crepitation around the cor solidatcd lung. The last sound is also distinct along the scapular region, and in the site of the saddle.

To have 20 drops of Arsenicum 1, every four hours.

On the 12th. - He is found lying - the first time since his illness; pulse 42 ; respiration 13 ; crepitation but slight; appetite much improved.

On the 13 th. - The pulse is 44 ; the breathing 12 ; there is a peculiar sharp, but not strong jerk of the heart at every contraction ; all the crepitation has disappeared, and the respiratory murmur has returned; so also has it in the lately bepatized lower third of left lung, where it is mingled, here and there, with loud crepitation; the horse eats and drinks freely, and looks lively. In all other respects there is a decided improvement. Continue medicine.

In the evening of the same day, there is no great change manifest, except that at every beat of the heart there is a peculiar sound, similar to what may be produced by striking a piece of tin with a sharp pointed rod of iron.

To have 20 drops of Digitalis 1, every four hours.

On the 14th.-The ringing sound is gone; the pulse 33 and of 
its ordinary character; breathing 11 per minute; no abnormal sounds in lungs; in all other respects well.

To have two more doses of Digitalis, and then discharge the patient cured.

\section{6.-Abscess.}

As a result of pneumonia, a collection of matter is not uncommon. If a bronchial tube opens into the abscess, the matter, which is of a very offensive, stinking character, is coughed up more or less abundantly, and loud, gurgling sounds are heard in it on listening at the chest.

Some horses recover; whilst others linger on, never pick up flesh, won't eat, and die.

\section{Case.}

In the spring of 1867 , I treated a case of this kind in a horse belonging to Messrs. Mowlem, Burt and Freeman, contractors. The attack was one of severe inflammation of the lungs. An abscess formed and burst into a bronchial tube, whereupon the breath became foul, and offensive matter was coughed up. The animal was convalescent in 30 days. The chief medicines given were Aconite, Bryonia and Phosphorus in the early stage, and Ammonium Causticum and Hepar Sulphuris in the later. I also used, after the abscess broke, a steam fumigation medicated with Baptisia.

\section{7.-Bleeding from the Lungs.}

Rupture of a blood-vessel in the lungs, and discharge of blood by the nose and mouth, is an occasional occurrence in the horse, almost always as the result of severe and long-continued exertion; more rarely from heart disease. A horse severely run with hounds may be fatally attacked in this manner. 


\section{CASE.}

On October 2d, 1863, a horse belonging to Mr. Jay, contractor, immediately after strongly exerting himself in pulling a cart out of a clayey place, was suddenly seized with coughing, and brought up a quantity of florid blood, and some ran out of his nose likewise. He continued his work up to the 6 th, when he was again seized in the same way. 1 saw him next day. His pulse was 72 and soft; his countenance was anxious and dejected; his breathing somewhat hurried; loud rattling could be heard in the windpipe and large bronchial tubes; he shivered; and after coughing, blood was discharged. Five days afterwards, nothing ailed him. The medicines he had were Hamamelis and Bryonia, in 20 drop doses every three hours.

In such cases, Aconite and Arnica are useful.

\section{8.-Hydrothorax-Dropsy of the Chest.}

\section{IN ALL ANIMALS.}

A collection of fluid in the cavity of the chest is a not unfrequent sequel of pleurisy. When this disease is treated by bleeding and other lowering measures, dropsy of ths chest is almost unavoidable, especially if the horse has been in low condition previously.

There are cases without previous pleurisy, where, from poverty of blood and general weakness, fluid collects in the chest, in the sheath, at the breast, in the legs, \&c.

The symptoms are as follows:-All the acute symptoms of pleurisy have declined, and to all appearances the case is doing well. But on applying your ear to the affected side you discover that above a certain point you can hear the sound of healthy breathing, and below no 
sound at all. The higher the fluid rises, the more difficult becomes the breathing; the pulse is weak; the appetite lost; swelling appears under the skin at the sheath, breast, belly, legs, \&c., and unless relief be afforded, symptoms of exhaustion set in, followed by death.

\section{Treatment.}

Very frequently these cases do well; the fluid is absorbed and complete recovery takes place.

The best medicines are :-

Arsenicum when the breathing is difficult; the pulse weak; the appetite gone; the urine scanty; and the breast, legs, \&c., swollen.

Digitalis when there are indications of heart disease; pulse feeble and irregular, \&c.

Mercurius when the breathing is much oppressed; the nostrils dilated, the cough short and frequent.

Ferrum is of value in those cases which occur in debilitated subjects, and after bleeding.

China may prove useful under similar circumstances.

Doses.--20 drops for horses and oxen; 10 for sheep and pigs.

Repeat each dose every two or three hours.

Tapping the chest and evacuating the fluid has been resorted to in the horse with some success.

\section{9.-Chronic Cough.}

\section{IN SHEEP.}

This is invariably the result of mismanaged acute inflammation (in cases in which life is not immediately 
extinguished by them). It consists of a subacute inflammation, which proceeds ultimately to tuberculous decay. There are few sheep which are not more or less affected by it; but, inasmuch as they are despatched to the shambles very young, there is not much time for the disease to waste away or deteriorate the flesh. The cough will continue very long without affecting the appetite, but after a time it becomes more severe, the membranes lose color and become pale and flaccid, the caruncle and the vessels of the eye have no longer the characteristic redness of health, and afterwards loss of flesh, dullness, languor, \&c., slowly and gradually supervene. In such cases the disease has changed its character, and has degenerated into consumption.

\section{IN HORSES.}

It may either be the result of any of the foregoing acute diseases affecting the respiratory apparatus, or of worms in the intestinal canal, the irritation of which reacts upon these organs. It accompanies thick wind and broken wind, which proceed from the first of the above named causes. Excess of dry food will always aggravate it. Cough arising from permanent affections of the lungs: it will be provoked by eating; from irritation in the air cells,-and drinking will induce a fit of coughing: if from worms, we may notice the appearances which are usually caused by those insects, which may be distinguished in the dung; and the cough will be rough, hollow, and sonorous. Pressure of the throat externally will always provoke coughing, when the air passages are the seat of the disease, but not invariably so when they arise from worms. 


\section{a Case of Chronic Cough.}

May 3d, 1875, I was called to see a mare belonging to Edward Parmalee, of Auburn, N. Y., which had been treated for a long time by allopathic veterinarians, without success, or the least improvement.

Symptoms.-Bad cough, caused by the secretions from the head passing down the back passage into the throat, accompanied by every symptom of the heaves.

Treatment.-Commenced giving Arsenicum and Phosphorus, 20. drop doses, once an hour alternately. In about 12 hours the groom reported to me that the mare was coughing herself to death. I then ordered Caustic Ammonic to be given every fifteen minutes, until the cough was relieved.

On the 4th, again gave Arsenicum and Phosphorus, as before. Also, added to a pailful of water, every time the mare drank, two drops of a preparation composed of one ounce Laudanum and two drams Oil of Vitriol.

On the 10th, the cough was sensibly relieved, and the mare commenced to improve in flesh. On the 31st, cough had entirely ceased, she breathing naturally, and to all appearance as healthy as ever, and continues so up to the present time.

\section{Certificate.}

This certifies that Ed. Parmalee's mare was in my care from January 21st to May 23d, 1875. On the 23d day of January she commenced coughing, which continued to increase until the 26th of April. During this time she was attended by two allopathic veterinarians, without improvement, but steadily grew worse.

Within 24 hours after Dr. Colby was called, the mare began to improve, and on the 5th of May was so far well as to be taken home by her owner. After he doctored her four weeks, she was entirely recovered, and is still sound.

June 16, 1875.

Wm. Remington. 


\section{0.-Thick Wind-Broken Wind.}

THIck WIND is likewise, in general, the result of acute inflammation, and more especially in the air-tubes, which become permanently obstructed by the incrustation or enlargement of the membranes. It may also be the result of fatness or malformation of the chest, but rarely, if ever so, in highly bred or fleet horses. It consists of very rapid, short, and difficult inspiration and expiration (in very quick succession), and will generally be followed by

BRoKen WIND, if the animal be put to severe exertion. Broken wind is identified not so much by the rapidity of the inspiration and expiration, as by the second effort which attends the latter, (being effected by two puffs as it were); whereas, the former (inspiration) is unaltered.

Apart from the occurrence of broken wind as the immediate result of obstruction of the air-cells (thick wind), from previous and more remote acute inflammation, broken wind very often occurs amongst heavy draft horses, especially as the result of bulky feeding-strawyard feeding, \&c.-(the excessive proportion of fodder yielding little nourishment, and of which the animal consumes a great deal) particularly if the animal be habitually put to work directly after feeding.

\section{1.-Roaring, Wheezing, Whistling.}

Roaring consists of loud, sonorous breathing, provoked by exertion, (accelerated pace) especially up an acclivity, and is characterized by a peculiar groan, ut- 
tered by the animal when suddenly struck or threatened. It may result from purely local inflammation, followed by thickening of the membranes, or from the extension of the inflammatory action of strangles to the air-passages, or from other like causes, or from a peculiar distortion of the upper part of the windpipe, occasioned by the strain of a tightened bearing-rein.

Wheezing is an affection sufficiently explained by the name, but in which the noisy breathing is continual, in rest as well as in motion. It is more directly the result of bronchial inflammation, and will often remain after it as a chronic affection.

Whistling is also explained by the name, but it is observable only after some continuance of rapid motion, particularly up an acclivity.

\section{Treatment.}

Treatment, in the majority of these chronic affections of the respiratory apparatus, resolves itself into

Regimen.-The food of a horse affected with chronic cough (proceeding from the lungs or air-tubes in particular) should consist as much as possible of a large porportion of fresh food of the better qualities. Early tares are very advantageous. Means should be sought to prevent it from eating the straw, and very little dry cut fodder should be afforded. The water should never be given quite cold. Transitions of temperature should be as much as possible avoided, as well as undue exposure. Ample ventilation, and regular exercise, without sudden and violent acceleration of pace, are essential.

The thick-winded horse should never be overfed, (ats is common, under the mistaken idea of providing for a 
long journey) nor should it be put into motion immediately after eating; regular and sufficient exercise, and other precautions, as just stated.

'The broken-winded horse should be fed upon highly nourishing food, of small compass, \&c., so as not to distend the stomach, and communicate any pressure from the diaphragm to the lungs, \&c. ; carrots are very beneficial. The other directions as just stated.

For worm-cough select from Belladonna, Bryonia, Dulcamara, Cina, Hepar s., and Sulphur.

For cough after eating, from Arsenicum, Baryta c., Calcarea c., Carbo veg., China, Kali carb., and Phosphorus.

For cough after drinking, from Aconitum, Arsenicum, Drosera, Hepar s., Lachesis, Ammo. mur., and Spongia.

For thick wind, from Arsenicum, Cuprum a., Ipecacuanha, Lachesis, and Tartarus emeticus.

For wheezing, and perhaps also whistling, from Belladonna, Carbo veg., Drosera, Dulcamara, Hyoscyamus, Iperacuanha, Phosphorus, Sambucus, Spongia, Veratrum, and Nux Vomica.

For the pulmonary fever, or "rot of the lights" in sheep, when assuming a low type, consult Arnica, Ipecacuanha, or Opium; and give the medicine whose symptoms best describe the case ; or two or more of these may be given in alternations.

D osE. -12 drops once in three or four hours.

\section{2.-Cough in General.}

Cough is the most common, the most obvious, and in some one of its forms the most universal symptom which presents itself in inflammations of the lining membrane 
of the trachea or windpipe, of the bronchial tubes, of the lungs, and of the pleura, or serous membrane which incloses them. In some chronic forms of these diseases, and in some still more obscure disorders of the respiratory apparatus, cough is almost the only indication of the abnormal condition. Hence, it requires to be studied very carefully by itself; and for this reason we have taken pains to present, in this section, both a thorough description of the varieties of cough, and a careful statement of the characteristic cough symptoms, and other obvious indications of the chief remedies which have been found useful in the coughs of domestic animals.

The varieties of this symptom, as dry, loose, \&c., taken in connection with the accompanying conditions, greatly aid in selecting the remedy suited to the case. It is not, indeed, always possible, especially for an inexperienced veterinarian, or still more for a lay practitioner, to determine whether, in a given case, there is bronchitis or pneumonia; but if the symptoms are carefully compared, and the medicine selected in accordance with them, the patient will be in a fair way to improve, notwithstanding the uncertainty of the surgeon's diagnosis. And on account of the immense importance of the cough. symptom, in deciding the character of the illness present, we place before our readers the following very full discussion of the various kinds of cough, communicated by W. C. Lord, F. R. P. S., to the British Journal of Homcopathy, and from the same authoritative source we subsequently quote the symptomatic indications of remedies in cough, to which, also, are added others suggested by our own experience and observation, and from other authorities. 


\section{3.-Classification of Coughs.}

Idiopathic, which seems to exist independent of any other disease, cough being the prominent and only symptom.

Symptomatic, when the cough depends on or accompanies other affections, such as bronchitis, catarrh, \&c.

These may again be subdivided into acute and chronic cough.

The acute cough is of recent origin; is frequently symptomatic, and usually disappears with the disease to which it owes its origin.

The chronic cough is either a continuation of the acute for an indefinite period, or has been idiopathic from the commencement.

The causes are irritability or inflammation in some part of the mucous membrane lining the air-passages or lungs; nervous derangement, especially of the pneumogastric and ganglionic nervous system; dentition; organic changes in the thoracic viscera, or the passage of some foreign body into the respiratory tube.

To distinguish the seat of cough and its cause is a subject of the greatest importance to the scientific practitioner, who places more confidence in the pathologic state of his patient, and the well known local action of drugs, than in mere symptom covering. I will, therefore, endeavor to explain (although a very difficult task) the diagnostic symptoms attending the various kinds of cough to which the horse is liable, and afterwards point out the homøopathic treatment which I have found most successful. 


\section{4.-The Cough from Dentition.}

This is loud, clear and ringing, heard chiefly in the morning and at night; is met with in young horses, especially between four and five years old, when it seems to depend on nervous irritation, produced by the canine teeth or tusks coming up; the mouth is hot, and the bars of the palate full, as in "lampas." The horse evinces a certain degree of tenderness in eating his corn, and takes longer time about it than usual, yet his spirits are good and the general appearance healthy.

\section{5.-The Pharyngeal Cough.}

It arises from irritation or inflammation of the pharynx, fauces, or neighboring glands; is not clear and loud, but moist, heavy-sounding, long, and as it were, hanging in the throat. This cough may be short and dry at the commencement, but only remains so until the secretions return, which they soon do, and in increased quantity.

\section{6.-The Laryngeal Cough.}

This generally depends on irritation of the membrane lining the larynx, but sometimes on derangement of the recurrent nerve. It comes on in paroxysms, and is easily produced by compression on the top of the windpipe.

When caused by a dry state of the membrane, this cough is short and dry, has a hard, ringing or metallic sound, immediately followed by a long, harsh inspiration. 
When caused by derangement in the recurrent nerve, this cough is loud, dry and spasmodic, and frequently chronic. As soon as the secretion returns to the membrane, the cough becomes loose and less painful ; instead of the cooing noise heard on applying the ear to the larynx, in the primary or dry stage of the membrane, a mucous rattle will now be heard. These sounds will help us materially in forming correct diagnosis.

\section{2\%.-The Tracheal Cough.}

If the secretion from the membrane lining the trachea be increased, the cough will be prolonged and moist, sometimes followed by a thick, white discharge from the mouth or nose. In this case the ear applied to the trachea will hear the mucous rattle, and compression will cause the animal to cough. If, on the contrary, the membrane be dry, so will the cough, and on applying the ear to the windpipe no moist sound will be heard, as in the former case, but instead, either a shrill cooing or base sound-according to the size of the tube from constriction or deposition of lymph, and the dry state of the membrane. I have cured a case of tracheal cough, of long standing, where the aperture in the lower part of the tube was so small that a whistling sound could be heard during inspiration, and the cough was short, hacking, and feeble.

\section{8.-The Bronchial Cough.}

This cough, at first short, hard, dry, and frequent, soon becomes muffled, moist and prolonged, when the 
secretion returns. The absence of irritation in the upper part of the air-passages leads us to investigate the lower portion by auscultation, which alone can give a correct diagnosis. When the cough depends upon inflammation and dryness of the membrane lining the large bronchi, the ear or stethoscope applied to the hollow in front of the chest detects a cooing sound or rhonchus; but should the small bronchi be similarly affected, the ear applied to the sides of the thorax detects the dry or sibilant rales. As soon as the secretion returns and the cough becomes loose, the mucous rattle will be heard with diminished and often suppressed respiratory murmur, until the secretion becomes removed by a cough. These abnormal and suppressed natural sounds, however, belong to symptomatic rather than idiopathic bronchial cough, which is distinguished by negative rather than positive symptoms, such as absence of fever, sore throat, or any other catarrhal symptoms; compression on the larynx or trachea, such as will produce laryngeal and tracheal cough, has no effect on this cough, which depends upon an irritable (not inflammatory) state of the bronchial membrane, and yields generally in two or three days to the homopathic treatment, which I shall presently describe. The idiopathic bronchial cough is loud, dry, and comes on in fits or paroxysms, at uncertain periods of the day, causing the animal considerable annoyance, but not interfering with the general health or appetite.

\section{9.-The Pulmonary Cough.}

This is generally symptomatic of inflammation in the substance of the lung, or of some organic change, the $9^{*}$ 
result of previous inflammation. It is usually short, dry, and frequent, accompanied in the former case by dyspnœea, and increased by percussion. As the inflammation increases, this cough becomes more constrained and painful, and ceases altogether. When inflammation is present in the parenchyma, auscultation detects the crepitant or other rales indicative of pneumonia. In chronic pulmonary cough, when organic change has taken place, it is usually indicated by absence of respiratory murmur in one or more circumscribed portions of the lung.

\section{0.-The Broken-winded Cough.}

This cough, which usually betokens vesicular dilatation and interlobular emphysema, is always symptomatic of a morbid state of the nervous system, which it is necessary I should fully explain, in order that the treatment which has proved successful may become intelligible to the reader. The cough is generally at first spasmodic and paroxysmal, but soon becomes short, single, and so feeble that it can scarcely be heard at a few yards distance. To diagnose this cough is very easy, for not only is it peculiar in character, but it is accompanied by dyspnoea, and a double expiration or supplementary effort performed by the abdominal muscles. This is attributed, by nearly all foreign authors, to an emphysematous state of the lung, requiring this supplementary expiration to expel the extravasated air from the tissue which they suppose has been ruptured. Several veterinary surgeons, however, declare that they have examined broken-winded horses in which there was no 
emphysema (D'Arboval, Rodet, Volpi, Professors Dick and Sewell). In those cases which I have examined I have found the lungs much paler, and not collapsed, as usual, when exposed to atmospheric pressure.

I have also observed dilatation of the small bronchi and air-cells, as well as interlobular emphysema, but have not been able to trace the latter to rupture of the cell. By some, a thickening or other altered condition of the membrane lining the air-passages has been observed, which has been put down by Laennec as the cause of the cough, and the latter as the cause of the dilatation and rupture of the air-cells, which he assumes does exist. The pathological changes which have been (though rarely) seen, are atrophy of the heart, and lesion of the diaphragm. Dupuy, one of our best French authorities, first conceived the idea that it was a nervous affection, and said that he had produced symptoms somewhat similar by compression and section of the pneumogastric nerves; but English authors, with very few exceptions, agree with Percival, that "emphysema of the lungs is the true pathology of broken wind." Professor Gamgee, however, dissents from this doctrine in the following words: "In stating our opinion on the nature of broken wind, we unhesitatingly affirm that it is at first a purely nervous disorder, dependent on the condition of the digestive organs, and in which the pneumogastric nerve is especially involved. As the result of a cause which thus operates through the nervous system, dyspnoea ensues, and organic lesions soon follow, and most frequently in the shape of pulmonary emphysema." All these theories are no doubt highly interesting. but in a curative point of view of little importance to the al- 
lopathic practitioner, whose treatment would be just the same whether the disease of which the cough is symptomatic consisted in emphysema, thickness of the membrane, dilatation of the air-cells, or nervous derangement. It is, however, of the utmost importance to the homoeopathic practitioner to ascertain the fons et origo of this cough, which I shall now endeavor to explain.

In my opinion, not only the cough but all the attending phenomena depend upon loss of power in the nerves furnished to the lungs by the anterior and posterior pulmonary plexuses; and on a similar derangement in the gastric branches of the par vagum must depend those symptoms of indigestion, which are so frequent an accompaniment of the disease. This loss of power in the branches supplied by the pulmonary plexus, which are nearly all ganglionic, produces a degeneration and loss of power not only in the muscular coat of the bronchial tubes, but also in the elastic tissue of the lung, upon a healthy state of which principally depends the act of expiration. In consequence of this want of elasticity in the pulmonary tissue, the cells became unnaturally dilated, and this dilatation, or impurity of unchanged air, produces the short, single cough, rendered feeble by the loss of power in the phrenic nerves and in the muscular tissues clothing the respiratory tubes. As these are unstriped muscles, it is clear that the ganglionic system is at fault, and to it, not to the pneumngastric nerve (as taught by Professor Gamgee), must we turn our attention and apply our curative agents. This theory of loss of nerve power and elasticity will account for the double expiration, not only in those cases in which emphysema exists, but also in those cases in which no such 
lesion has taken place, for in both the abdominal muscles are called on to assist, by a supplementary effort, the weakened pulmonary elastic tissue.

\section{1.-The Consumptive Cough.}

This is very rarely met with in the horse, and may be diagnosed by absence of murmur in circumscribed spots or patches of one or both lungs, bronchial respiration, and cavernous or spumous rales. This cough denotes tubercles in the lungs, which usually terminate in glanders.

\section{2.-The Chronic Cough.}

This is so named from its lasting for months or years without appearing to injure the animal's health; may be an effect of previous disease in some part of the respiratory organs, or it may be idiopathic, when its origin must be ascribed to nervous derangement.

There are three kinds of chronic cough, namely:

1. The hollow, groaning cough.

2. The loud, dry, spasmodic cough.

3. The short, hacking, feeble, grunting cough.

The first, or hollow cough, which seems to come from the inmost recesses of the body, is preceded by a groan, or made up of half groan, half cough. It comes on early in the morning or at night, and does not interfere with the general health for a very long time. Auscultation sometimes fails to detect the seat of this cough, when 
I conclude that it arises from some derangement in the pulmonary and gastric branches of the par vagum.More frequently, however, it depends upon consolidation of a portion of the lung, which may be diagnosed by the absence of murmur, and dulness on percussion over the hepatized portion.

The second, or spasmodic cough, seems to me to depend upon some derangement in the recurrent nerve, or on an irritable state of the membrane lining the larynx. It is generally heard when the horse is eating, drinking, or first brought out of the stable.

The third, or grunting cough, is similar to the brokenwinded one, but unattended by the jerking movement of the flanks, or any emphysema. The pathology of this cough only differs from that of broken wind insomuch as the elasticity of the lung is concerned; for in this case the pulmonary tissue does not appear to be affected at first, although it eventually becomes so, and then the animal is broken-winded.

But the organic nervous system supplying the lining membrane and also the diaphragm is equally depressed, and as a natural consequence, we find an alteration in the bronchial secretion, or some deposition of lymph in the air-passages, giving rise to a cough which is short, feeble, and devoid of resonance, owing chiefly to loss of power in the phrenic nerves. A strong forcible expiration or cough requires a sudden inspiration, which cannot well take place if the diaphragm is out of order.This has been confirmed by Girard and other veterinary surgeons, who observed a cough, similar to the one under consideration, in horses that they afterwards found had sustained a lesion of the diaphragm. 


\section{Remedies for Cough.}

Aconitum Napellus may be used in the primary stages of all inflammatory coughs, whether seated in the mucous membrane or pulmonary tissue. Its principal indication in the former is dryness of the membrane. In the latter, increased and full or strong pulse, with dyspnœa and other indications already given in the diagnostic symptoms of pulmonary coughs. The Aconite cough is short, dry, frequent, and generally spasmodic. As soon as the inflammatory symptoms subside, which is denoted by increased mucus or muco-purulent secretion, the Aconite should be either discontinued or given in alternation with the drug specifically adapted to the nature and seat of cough under treatment.

In human medicine this drug holds a high position as a remedy for coughs, but, according to my experience, it can only be regarded as a useful auxiliary in veterinary practice. From its extensive range of action, Aconite may be useful in those recent, hard, dry coughs, whose seat it may be difficult to diagnose.

Aconite will be indispensible in the first or inflammatory stage of bronchitis, of pleurisy, and of pneumonia, which are characterized by hardness of the pulse, chilliness, and high fever. In the last named disorder it will be indicated by the pulse being quickened and full, the breathing labored and panting, the mouth hot and dry, and the membranes of the eye and nose injected.

Dose. - 20 drops may be thoroughly mixed in a pint of water; of this 2 tablespoonfuls may be given to the larger animals, 1 to the medium sized, as the sheep, the goat; and 1 or 2 teasyoonfuls to the sinaller domestic patients; the dose to be repeated once in one, two, three, or four hours, according to the severity of the symptoms; diminishing the frequency of repetition as the case improves. 
Apis Mellifica acts especially on the lining membrane of the larynx, and seerns to control a state of inflammation in it even greater than that to which Belladonna is applicable-one in which I infer, from the symptoms, that submucous infiltration has taken place. The cough is suffocative, painful, and not so hard as the Belladonna cough; but there is usually more dyspnœea, which leads me to diagnose a thickening of the membrane, probably from effusion, which Apis speedily removes. It has also, in my hands, quickly removed a painful, hoarse cough, accompanied by a clear, ropy discharge from the mouth, similar to the symptoms indicating Mercurius, which appears to me to be its chief analogue as far as the action of both on the larynx is concerned. Apis has also been used by me, with considerable success, in some cases to which Belladonna appeared to be homœopathic, but yet failed in removing the cough.

Dose.-See Aconite, page 199.

Arsenicum Album acts on the mucous membrane lining the whole of the respiratory tract. It is chiefly indicated in those coughs which remain after influenza, or catarrh of an asthenic type, accompanied by an unhealthy appearance of the coat. The cough is usually dry, but with watery discharge from the nostrils, and excited by exposure to cold air or drinking cold water. It would seem to be dependent on loss of power in the organic nervous system, giving rise to a thin, acrid secretion from the respiratory mucous membrane, to cure which it only requires that the vaso-motor system be raised to a healthy standard by the action of Arsenicum. On referring to my pathology of "broken wind," the reader will understand why Arsenicum has been so successful in the hands 
of M. Hew, of Chaumont, in curing this disease. This gentleman has lately published a report of ten cases of broken wind cured by Arsenicum. He gave it to the extent of fifteen grains daily, and at the end of a fortnight not only the cough but all other symptoms had disappeared. Chronic cough with thick wind is also one for which Arsenicum is peculiarly suitable; but in these cases I would recommend the Liquor Arsenicalis in thirty drop doses.

In pneumonia, Arsenicum will be indicated in extreme cases, when there are great depression; weak pulse; cold, clammy mouth; purging, and entire absence of appetite; cough worse at night (after midnight), on going up a hill, and especially when attended with difficulty of breathing. This is the principal remedy for heaves or broken wind, as above stated.

DosE.-Same as directed for Aconite, page 199, but repeated only half as often.

The chief sphere of action of Belladonna, as a cough medicine, is the upper portion of the air-passages, including the pharynx, fauces, and larynx, as well as the neighboring glands. I have never witnessed any beneficial effects from Belladonna in bronchial coughs of a sthenic character, but in those low, feeble, painful coughs, having their seats in the bronchial membrane, and accompanied by fever of an asthenic type, I have used Belladonna in alternation with Bryonia most successfully.

Belladonna has proved remarkably successful in the dry cough of the late epizootic; it is indispensable in pneumonia, and here, as in the epizootic, it may be given in alternation with Phosphorus to great advantage.

Dose.- Similar to Aconite, page 199, but not so often repeatcd. 
Bryonia is a very valuable cough medicine, but the state for which it is applicable has not, according to my experience, been so well explained by any author as by Dr. Hughes, in his Pharmaco-dynamics. Its sphere of action as regards coughs is the larynx, trachea, and large bronchi, but it has little or no effect on a cough having its seat in the small tubes. It does not appear to me to be applicable to an active state of inflammation, but to irritability, producing " continued, dry cough, especially early in the morning." The Brionia cough, although dry, is usually accompanied by a rattle in the throat or some part of the trachea. This rattle is not such as ordinary mucus would produce, but conveys the idea that plastic lymph has been thrown out, or mucus of a tenacious character. Pressure on the part of the trachea where this rale is heard inmediately produces the cough, as does also exposure to cold air or exercise. In those coughs which prove a sequel to some active disease in the air-passages, which has left the membrane in an irritable state, giving rise to cough when the horse is first brought out of the stable, Bryonia will be found a most useful remedy; but when any soreness of the throat is present it must yield in value to Belladonna. Bryonia is required in pleurisy, where it will be indicated by the aggravation on motion and tenderness on pressing upon the flanks, and by a full and moderately hard pulse.Short and restrained cough, as if it were painful, and the breathing short and catching, will especially call for this remedy in pleurisy. In bronchitis, Bryonia will be indicated by lout rattling from the first, heard when the ear is applied to the breast or side; cough is lonse and attended with discharge of frothy phlegm; the respira- 
tion is quick, difficult; there is rattling of mucus in the windpipe, in the air-tubes (bronchia), and in the lungs; or the cough may be frequent, dry, irritating (Aconite may here be preferred); there is a discharge of thick phlegm. Aconite and Bryonia have been advised to be given in alternation, in the earlier stages of this disorder.

Dosf. -12 to 20 drops, once in two or three hours, in the most urgent cases (e. g., of pleurisy); usually to be prepared as directed for Aconite, page 199.

I have found the Tincture of Iodine useful both for laryngeal and tracheal coughs, attended by an effusion from the membrane of a plastic nature, as well as in those cases dependent on chronic inflammation of the membrane, and accompanied by an unhealthy discharge from one or both nostrils. In its action on the laryngeal and tracheal membrane it resembles Bryonia, but differs from the latter in its affinity for the salivary glands.When the cough depends upon an irritable, not inflammatory, state of the salivary glands, or of the mucous follicles in the laryngeal and tracheal membrane, whereby these secretions are changed from their normal character, I believe. Iodine will be found superior to Mercurius, Bromine, or Spongia, but often inferior to Arsenicum, which appears to be its chief analogue.

Dose.-As directed for Aconite, page 199, repeated once in three or four hours.

The Mercurial preparations act on the mucous membrane lining the air-passages, but principally on the laryngeal portion, and also on the salivary glands. I have very little faith in Mercurius as a remedy for bronchial coughs, either acute or chronic, and feel convinced that if those authors who recommend it for chronic bronchi- 
tis would substitute Antimonium tartaricum either in trituration or dilution, they would find the latter a far superior remedy, especially in those cases where the tubes are filled with mucus. The cough requiring Mercurius is dependent on the secondary stage of inflammation in the pharyngo-laryngeal region and neighboring glands, and its use is indicated by increased secretion, nasal discharge, and sore throat.

Nux vomica. - Tinct. nucis is principally indicated in coughs of a nervous and spasmodic character, which some horses have early in the morning and at night, but without deranging the general health, and often without any pathognomonic symptom whereby its seat can be accurately determined. This chronic cough appears to me to depend upon some derangement in the pneumogastric nerve. Sometimes the recurrent is the branch most affected, sometimes the pulmonary branches, but occasionally the gastric, when we have what is called a stomach cough. The acute symptomatic cough for which $N u x$ is applicable is attended by constipation or a slimy state of the freal balls, foul tongue, or a peculiar white appearance of its dorsum, and probably yellowness of the buccal membrane, and sometimes by that state of the skin called "hide-bound." A most remarkable indication for Nux Vomica in cough is found in breaking wind simultaneously with the cough. This we have observed on several occasions, in which $N u x$ never failed to cure. In coughs attended with a disposition to heaves, and where the animal immediately begins to cough on being made to trot, $N u x$ will be found invaluable. Coughs worse on going down hill, especially trotting, which seems to irritate the bronchia by a sort of 
downward mechanical pressure, compare Ammo. mur. Dr. James Moore, whose cough indications we have freely quoted, gives the following indications for Nix vomica: dry, hoarse, spasmodic cough, worse in the morning, and after eating and exercise, and especially when the stomach is disordered, the tongue furred, the mouth foul, the appetite variable, the bowels confined.

DosE.-As directed for Aconite, page 199, repeated once in three or four hours.

Phosphorus is the best medicine for pulmonary coughs of a febrile or inflammatory character, whether such cough has its seat in the small bronchi or substance of the lung. It is also useful for chronic cough arising from some organic change in the pulmonary tissue. The Phosphorus cough is dry, short, and frequent, or what is termed a "racking" cough. Human surgeons recommend this drug for laryngeal cough, but I cannot indorse this recommendation in veterinary practice. Necessary in pneumonia, especially in the second stage, when the lung is solidified, a condition which may be ascertained by observing the physical signs, of dulness on auscultation; and when the breathing is very labored and distressed, and when a reddish or yellowish discharge is coughed up.

DosE. - As directed for Aconite, page 199, to be repeated once in four hours.

Rumex crispus, whose specific action on man is confined to the laryngo-pharyngeal region, in the horse has an equally powerful effect on the bronchial membrane.

The laryngeal cough, which Rumex removes, is dependent on an irritable (not inflammatory) state of the membrane, either idiopathic from the commencement, or 
the result of previous inflammation, which has been removed by other means. In consequence of the absence generally of objective symptoms, and the horse being unable to give us the subjective ones, it requires a great deal of practical knowledge to decide on the seat and class of cough for which Rumex is suitable; but once correctly diagnosed, the remedy acts like magic.

There is no sore throat, bronchitis, or any marked constitutional derangement, and seldom any abnormal rale, yet the horse coughs frequently, especially on the slightest exertion.

Sometimes a sibilant or mucous rale may be heard, but more frequently the respiration is merely harsh, as though the membrane was deficient in secretion. It is, in fact, an idiopathic cough, whose seat it is difficult, and often impossible, to determine; but of this the reader may be assured, that if Rumex be the appropriate rem. edy, its action will be apparent in two or three days.

Dose.-As directed for Aconite, page 199.

So far Dr. Lord, except the directions for the dose; but there are still certain remedies, of less frequent occurrence perhaps, but still needed in the various disorders of domestic animals, whose titles are placed at the head of this section, disorders of which cough forms one of the most prominent and easily distinguished symptoms.

Arnica will be required in pleurisy resulting from external injuries, to be followed by Aconite, Bryonia, or Rhus tox., according to the existing conditions.

Cannabis may be required in inflammations of bronchia, or in pneumonia, where the animal is obliged to stand up in order to breathe.

Carbo veg.-Frequent attacks of hollow-sounding, 
spasmodic cough, occurring only in the morning. It is also indicated for cough in the evening till midnight.

China.-Cough at night, worse after midnight, difficult breathing, with debility, especially after hemorrhage from lungs (in human subjects).

Ammonium Causticum.-Much languor from the first ; coldness of the legs, ears, and nose ; rough, staring coat; quickened, difficult breathing; pulse small, weak, and frequent.

Sulphur will often be needed in pneumonia, and in pleurisy, when the acute symptoms have been removed by other remedies. It serves to prevent the cough from becoming chronic.

\section{DosE.-12 or 20 drops every morning.}

Antimonium Tartaricum (Tartar emetic) is indicated in bronchial cough, when the secretion (and discharge) of mucus is very copious, the cough is loose, the rattling loud, the breathing much distressed.

Kali bichromicum is indicated when the phlegm is tough, sticky, stringy, in old-standing cases, and when the tongue is covered with yellow fur.

Drosera.-Spasmodic, or chronic cough, hoarse, hollowsounding.

Spongia.-Sharp, shrill, ringing cough, tracheal inflammation, or dry, hollow, barking cough, sounding like croup or like whooping-cough in the human subject.

Ipecacuanha.-Quick, anxious, sighing breathing, suffocating attacks, frequent dry cough, suffocating cough, loose cough, rattling in bronchia, violent racking cough. Particularly recommended for sheep.

For the dose of these remedies see Aconite; they must be repeated according to circumstances. 


\section{3.-Pleuro-Pneumonia in Cattle.}

From Gamgee's "Dairy Stock," we take the following concise statement of the symptoms of epidemic pleuropneumonia in cattle: "From the time that an animal is exposed to the contagion to the first manifestation of the symptoms, a certain period elapses; this is the period of incubation. It varies from a fortnight to forty days. Some faith may be relied on reports of even longer periods of incubation. The first signs, proving that the animal has been seized, can scarcely be detected by any but a professional man; though, if a proprietor of cattle were extremely careful, and had painstaking individuals about his stock, he would invariably notice a slight shiver usher in the disorder, which for several days, even after the shivering fit, would limit itself to slight interference with breathing, detected readily on auscultation. Perhaps a cough might be noticed, and the appetite and milk secretion also diminish. The animal becomes costive, and the shivering fits recur. The cough becomes more constant and oppressive, the pulse full and frequent, usually numbering about 80 per minute at first, and rising to upwards of 100 . The temperature of the body rises, and all the symptoms of acute fever set in. A moan or grunt, in the early part of the disease, indicates a dangerous attack, and the alæ nasi, or nasal cartilages, rise spasmodically at each inspiration; the air rushes through the inflamed windpipe and bronchial tubes, so as to produce a loud, coarse respiratóry murmur; and the spasmodic action of the abdominal muscles indicates the difficulty the animal experiences also in the act of expiration. Pressure over the intercostal 
spaces and pressing on the spine, induce the pain so characteristic of pleurisy, and a deep moan not unfre. quently follows such an experiment. The eyes are bloodshot, the mouth clammy, skin dry and tightly bound to the subcutaneous textures, and the urine is scanty and high-colored.

"On auscultation, the characteristic, dry, sonorous rale of ordinary bronchitis may be detected along the windpipe and in the bronchial tubes. A loud sound of this description is, not unfrequently, detected at the anterior part of either side of the ehest, whilst the respiratory murmur is entirely lost posteriorly, from consolidation of the lung. A decided leathery friction-sound is detected over a considerable portion of the thoracic surface. As the disease advances, and gangrene, with the production of cavities in the lungs, ensues, loud cavernous rales are heard, which are more or less circumscribed, occasionally attended by a decided metallic noise. When one lung alone is affected, the morbid sounds are confined to one side, and on the healthy side the respiratory murmur is uniformly louder all over.

"By carefully auscultating diseased cows from day to day, interesting changes can be discovered during the animal's lifetime. Frequently the abnormal sounds indicate progressive destruction; but at other times, portions of lung that have been totally impervious to air, become the seat of sibilant rales, and gradually a healthy respiratory murmur proves that, by absorption of the materials that have been plugging the lung-tissue, resolution is fast advancing. I have seen some very remarkable cases of this description.

"Unfortunately, we often find a rapid destruction of 
lung-tissne, and speedy dissolution. In other instances, the general symptoms of hectic or consumption attend lingering cases, in which the temperature of the body becomes low; the animal has a dainty appetite, or refuses all nourishment. It has a discharge from the eyes, and a fetid sanious discharge from the nose. Not unfrequently it coughs up disorganized lung-tissue and putrid pus. Great prostration, and indeed, typhous symptoms set in. There is a fetid diarrhoea, and the animal sinks in the most emaciated state, often dying from suffocation, in consequence of the complete destruction of the respiratory structures."

The following symptoms are given by Dr. James Moore, as the characteristics of the malady which are of most frequent occurrence :

In the First Stage.-It begins in one of three ways.Firstly, it may attack the cow suddenly, and run a rapid course in spite of all treatment; secondly, it may come on slowly and insidiously, the cow appearing to be not very ill, whilst the lungs are becoming diseased beyond the hope of restoration; and thirdly, it sometimes begins with violent purging, followed by great weakness and loss of flesh.

The majority of cases, however, present the following symptoms: a short, dry, husky cough, which is heard only occasionally; it is highly characteristic of this disease, and when once heard cannot be mistaken again.The owner says, perhaps, that he has heard this "hoose" for two or three days, but thought no more about it. On inquiry, it will be found that the beast does not give so much milk as usual, and that it has a slightly yellowish 
tinge; the appetite is not much worse, yet still she is careless about her food, and does not lick her dish clean; when at rest, the breathing may not show any departure from its healthy play, but when the animal is moved and walked some distance, it becomes more frequent, labored, and difficult; the pulse is often bealthy in character, although sometimes it is weak, and slightly increased in frequency; the bowels may either be confined or purged, or quite regular; the body is sometimes hot, sometimes cold. The cow appears dull and listless; when at grass she separates herself from the others, and lies on the ground whilst they are browsing.

In the Second Stage.-The cough is now more frequent, and thick, frothy phlegm dribbles from the mouth; the breathing is short when the air is taken into the lungs, and long when it is pressed out of them; the inward breathing is attended with much pain, which causes the animal to grunt and to grate her teeth; the grunt is heard when the animal is pressing the air out from the lungs; the pain is much increased by coughing and change of position, and to lessen it the cough is now suppressed, or held back and short, and the cow stands fixed in one place. The pain is owing to the pleura being inflamed, and the position of the diseased place may be ascertained by pressing the side between the ribs with the point of the thumb; when pressed on, the animal will flinch and grunt. The pulse is quickened and oppressed ; the skin is hard, tight, and bound to the ribs; the horns are hotter, and the muzzle drier than usual; the head is lowered and thrust forward, with the nose poked out; the back is raised up; little or no food is eaten; the cud is seldom or never chewed; the milk is 
stopped; the bowels are bound, and, when moved, the dung is in hard, dry lumps.

In the Third Stage.-The breathing is much quickened, very difficult, labored, and even gasping; the breathing is carried on partly through the mouth, partly through the nostrils; the breath has a bad smell ; a stringy, frothy fluid constantly dribbles from the mouth; the cow groans loudly and frequently, whilst the grunt is either gone or subdued; the pulse is quick, weak, and in some cases imperceptible or intermittent; the horns, ears, and legs are cold, the skin covered with cold sweat, the head and neck stretched out, with the nose poked into the corner of the manger; the fore legs are separated from one another and fixed in one place, unless the cow is restless and uneasy; sometimes the hind ones are crossed over each other, or the hind fetlock-joints are knuckled forward; the stoppage of milk is complete; the animal is reduced to a skeleton; the strength is also, of course, greatly impaired, and the beast can scarcely cough; insensibility sometimes steals over her; the urine is very high colored ; towards the last, violent purging comes on, the discharged matter being quite watery, blackish, highly offensive, and sometimes mixed with blood; eventually, the cavity of the chest becomes so full of fluid, or so much of the lung is condensed, that the breathing, from being more and more difficult and frequent, at last ceases, and the animal is dead.

\section{Treatment.}

It is of the utmost importance that this should be begun at the earliest possible moment. The sooner the medicines are given after the first symptoms of the mal- 
ady begin to make their appearance, the more promptly and certainly may the disorder be removed.

Aconite.-Pulse hard and quickened; breathing short, painful, anxious, attended with groans and open mouth; shivering or trembling, attended with coldness of the legs and horns, and dry heat of the skin.

Dose.-20 drops in a little water, every one or two hours, according to the violence of the symptoms.

Bryonia.-Frequent, short, suppressed cough, which conveys to a looker-on the idea that the action of coughing causes a sharp pain in the chest, and the cow tries to lessen this by stopping or cutting short the effort of coughing. For the same reason the breathing is short, attended with pain and the characteristic grunt; pain, followed by flinching and grunt, when the ribs are pressed against; the animal remains standing in one place, and is unwilling to move, because the pain in the chest is thereby increased.

DosE.-20 drops every two or three hours.

It frequently happens that the cow presents all the symptoms which are included under the last two medicines, in which case both are to be chosen, and given every one, two, three, or four hours, not mixed, but time about, or in turns - that is, first one, then the other, one, two, or three hours after-then the first again, and so on, according to the violence of the symptoms. The same remark applies to all the other remedies. The reader, then, is to remember this rule: That in choosing any of the medicines, he must pick out those that correspond most nearly to the present symptoms of the cow.

Ammonium causticum.-Quick, difficult breathing, at- 
tended with rattling sounds; the breathing inwards is short, in consequence of pain; cough frequent, and attended with discharge of phlegm from the windpipe; great weakness, listlessness, and depression; pulse feeble and quickened; frequent shivering and trembling; skin hot and dry, afterwards moist.

Dose. -20 drops in a little water, every two hours, till improvement appears, then once in three hours only.

Lycopodium.-Fan-like motion of alæ nasi ; from this symptom, Dr. Wilson cured many cases with this remedy.

Arsenicum is the most suitable when there are wheezing, short, hurried, and difficult breathing; small, quick pulse; great weakness, and loss of appetite; grinding of the teeth; cold skin and clammy sweats ; frequent short cough; purging either in the first or last stage; then Arsenicum is the most suitable, and must be given as follows :

Dose. -20 drops every two or three hours.

If the following symptoms exist-breathing difficult, oppressed, and obstructed; pains in the chest, which are aggravated by taking a deep breath, by moving about, and by coughing; pain between the ribs ; frequent short cough, attended frequently with discharge of slimy phlegm, which is sometimes mixed with blood; violent purging, followed by wasting and weakness; then Phosphorus is required, and must be given as directed for the last medicine.

Sulphur exerts its beneficial action in this complaint, more especially when one or other of the foregoing remedies have subdued the more violent symptoms, and when the cow is slowly recovering. Its chief effect seems 
to be to confirm the tendency towards health, and to guard against a relapse. It is to be given as follows :

Dose.-20 drops in a wine-glassful of water, three times a day.

The food should consist of bran mashes, boiled turnips, and carrots, oat-meal or corn-meal gruel. Healthy cows should be removed from the vicinity of those that are sick. The latter should be housed in dry, well ventilated, and comfortable stables, and every care should be observed, by giving little food at a time, to prevent a fatal relapse after convalescence has been well established; for if the stomach is filled with food, the disease is very apt to return, and death is then the usual consequence.

By the aid of these hornœopathic medicines, Dr. Moore and other British veterinarians saved a very large portion of their cases in an epidemic of pleuro-pneumonia, so destructive that nearly all (36 out of 37 ) died under the ordinary treatment.

\section{4.-Spasm of the Diaphragm.}

I have seen several cases of a disease which, in my opinion, is correctly designated by the above name, and is often confounded with palpitation of the heart. "Nimrod" was the first to recognize and describe it. Since his day, others have published cases, and now there can be little doubt of its occurrence.

\section{Case.}

On August 31st, 1850, a mare belonging to Mr. Sidebotham, Manchester, was taken ill after a very severe galloping immediately after feeding. The symptoms were:- Pulse 64 , small and 
wiry; respiration 42 per minute; vessels of eye turgid; strong, spasmodic, irregular action of the diaphragm, from 45 to 50 per minute; breath drawn in forcibly, attended with a snuffling noise at the nostrils, and as rapidly expelled without noise; pulsation very distinct at each side of the back. To have 20 drops of Stannum, 1st dilution. An hour afterwards the pulse was 40, full and strong, and the spasmodic action far less powerful. Gave 10 drops of the same preparation. Two hours afterwards, the pulse was 30 , full and strong; now and then a slight spasm near the lumbur vertebræ. Repeated the medicine. Four hours later, the pulse was 36 , the respirations tranquil and natural, and all spasmodic jerking or pulsations gone.

Refer to the remarks on "Palpitation of the Heart," for the symptoms which distinguish the one disease from the other.

\section{5.-Parasites in the Lungs.}

\section{IN SHEEP, CALVES, \&c.}

The disease resulting from the presence of worms in the air-tubes and lungs is generally called hoose, or bronchitis. It prevails most in low, damp, marshy districts, and especially attacks young ruminants, when they begin to eat grass, although adult animals are not wholly exempt.

The first marked symptom is a slight, soft, and moist, or a dry, husky cough, which comes on in violent fits. The animal is dull and drooping, and occasionally rubs its nose on the ground or against a wall. The hair or wool is dry and harsh, very different from that of healthy animals. The breathing is quickened, difficult, and apparently attended with distress in the chest. Subsequently, in addition, the animal loses flesh and strength ; the eyeballs sink in their sockets ; the counte- 
nance expresses anxiety; the breathing continues difficult and grunting, and death occurs from exhaustion.

\section{Treatment.}

It is absolutely necessary to destroy the worms, which are a species of strongylus. Various means have been advocated. Perhaps the best is to cause the affected animals to breathe the fumes of burning Sulphur or Tobacco. The food must be generous, such as turnips, linseed cake, etc. As a means of prevention, turn the animals into a virgin pasture.

I have also observed good effects from Cina, 20 drops once or twice a day.

Give Arsenicum, 10 drops, and Carbolic Acid alternately, once in two hours in bad cases; in mild cases, three or four times a day.

$10 *$ 


\section{CHAPTER XI.}

\section{DISEASES OF THE HEART.}

Diseases of the heart frequently occur in the lower animals, but their detection during life, as well as the discrimination of one disease from another, is a matter of considerable difficulty, in consequence of the region of the heart being less accessible to examination than is the case in the human subject.

There are, however, a few diseases of this organ which can be made out with tolerable accuracy; to them I shall confine my observations.

\section{1.-Palpitation.}

Excessive action of the heart may occur in horses that are in feeble health and out of condition, or when strong horses have had a long run with hounds.

The symptoms are:-a dull, thumping noise proceeding from the interior of the body, and quite audible at a distance of some yards; this sound corresponds in time with the pulsations of the heart and the throbbing of the pulse. Sometimes, the heart's action is so energetic as to cause a jerking or shaking over the whole body; and the flanks are raised up likewise. 
Palpitation is sometimes mistaken for what has been called Spasm of the Diaphragm, in which a somewhat similar sound is heard. In such cases, according to my own observations, the sound could be heard a few yards off, and was very distinct on applying the ear to the back on each side of the spine. The breathing and pulse were both increased in frequency, and the thumps did not correspond with the heart's pulsations.

Horses are most liable to palpitation of the heart.

\section{Treatment.}

Rest the animal for a few days, be careful not to overwork him or drive him fast for some time afterwards, and pay attention to the diet.

If the stomach be disordered, treat as directed for "Indigestion," at page 90. In cases of palpitation, depending, as far as can be ascertained, on indigestion, Nux Vomica is especially suitable.

China should be given when there are symptoms of general debility, and poor appetite.

Aconite is especially required for energetic action of the heart, occurring in high-conditioned animals, after exertion, and attended with quick breathing.

Stannum, 1st dilution, has always cured the symptoms of spasm of the diaphragm described above.

Doses. - 20 drops for horses. Repeat the dose every one, two, or three hours, according to the urgency of the symptoms.

\section{2.-Enlargement of the Heart.}

An increase in the size of the heart is rather frequently. found in animals suffering from "broken wind." Such 
a disease affects the animal's powers of enclurance and speed; and, when the animal is over-driven, may bring on hœmorrhage from the lungs.

The symptoms are:-The action of the heart is heard and felt to be stronger than it ought to be, and extending over a larger space. In some cases, the stroke of the heart against the side is very strong. The increased action dependent on enlargement is constant, unlike that found in simple palpitation. In addition, a "clacking" sound is sometimes heard.

\section{Treatment.}

This disease is incurable. Regulating the diet, preventing overwork, and giving Aconite, Arsenicum or Digitalis, will give relief and prolong life.

Doses. -20 drops for the horse, three times a day.

\section{3.-Dilated Heart.}

This lesion may be known, as it occurs in horses, by symptoms which can hardly be mistaken. They are:Loss of appetite; languor ; cold legs and ears ; difficulty of breathing on the least exertion; giddiness or " megrims ;" small. soft, feeble, irregular pulse; feeble, tremulous action of the heart; and, in advanced cases, swelling of the legs, chest, and belly.

This disease is incurable.

\section{4.-Pericarditis.}

\section{IN ALL ANIMALS.}

This disease, which consists of inflammation of the scrous investment of the heart, is not unfrequent in all 
our domesticated animals. It may occur as an independent affection; more usually it is a complication of rheumatism, pleurisy, and pleuro-pneumonia of cows; and it has been known to follow a punctured wound.

The symptoms are often ambiguous, and are apt to be confounded with those of pleurisy. In a fully developed case, we find him standing still, anxious in the face, and evidently in great suffering; with his breathing much quickened and labored, and his pulse accelerated, small, hard, and sometimes irregular. Pressure on the left side, in the region of the heart, causes flinching and expression of pain. On listening at the same part we hear rubbing friction sounds, which cease when effusion or adhesion takes place; subsequently, the heart's sounds are muffled by the effusion. In unfavorable cases, the breathing becomes more distressing, especially on the least movement; the pulse feebler, and even imperceptible at the jaw; the eyes protruded and anxious; the legs and ears cold; dropsical swelling comes on in the legs, sheath, breast, \&c., and death speedily follows.

The late Professor Dick, in his "Manual of Veterinary Science," gives the following case:-_"I was lately consulted in a case of a mare which was taken unwell. She had left off feeding; her pulse was small and weak; her respiration scarcely affected; her mouth cool; her bowels regular; and her ears and legs fine, but cold. On moving her about in the stall, a twitching of the hind legs of the animal was observed, as if it was affected with cramp. On applying the hand to the left side, a peculiar pulsation was felt, as if the heart was moving in a fluid. This pulsating sound was readily heard on applying the ear to the left side, and was totally distinct 
from the sounds produced by water in the chest. The c.rso provel fat.l, and on dissection, the pericardium was found to contain four pounds of serum."

The following case is quoted from the "British Journal of Homœopathy," for 1858 :

\section{CASE.}

On April 5th, 1858, I visited a cart-horse belonging to Messrs. M urslınd, csal proprietors, Manchester, which has been under allopathic trextment for a week and is rapidly getting worse.

The most important symptoms are:-Pulse 120 per minute; violent jerking action of the heart; there is a peculiar ringing sound at every beat of the heart ; the respiration is 36 per minute; little breathing can be heard in the left lung, in consequence of the predominant action of the heart; the urine is voided frequently, and in small quantities, etc. This has been produced by a fly blister on the side. I had it washed off at once.

To have 20 drops of Digitalis, 1st dilution, every three hours.

On April 26th, pulse 104 and intermittent; respiration 20; the violent jerking of the heart is almost gone ; the respiratory murmur is now audible in the lower portion of the lungs, and some mucous rattles in upper part; the urine flows freely; the bowels are moved; the appetite is better, and the animal's appearance livelier.

To have 20 drops of Helleborus and of Arsenicum, 1st dilution, every three hours alternately.

On April 27th, pulse 95, still intermittent, but more distinct at jaw; respiration 10 per minute; the urine is profuse in quantity, and has a peculiar smell - the same as that which proceeds from the chest when opened after death from hydrothorax; in all other respects better.

Continue same medicines.

On the 28 th, pulse 80 ; from 16 to 20 of the beats in the minute are slower, the rest quicker than in health; respiration normal; appetite still improving and better otherwise. Continue as before. 
On the 29th, the same, except that the pulse is fuller and softer.

Substitute Spigelia for Helleborus.

On May lst, pulse 60, full and strong; better otherwise.

Continue same medicines.

On May 7th.-Since last report has been steadily improving in every respect; pulse 54 ; appetite good; has been resting.

To have Sulphur three times daily.

On the 12 th all right. 


\section{CHAPTER XII.}

\section{DISEASES UF THE NERVOUS SYSTEM.}

\section{1.-Rabies - Hydrophobia. \\ IN DOGS.}

Canine madness occurs spontaneously in the dog, wolf, and fox, and in other animals as the result of contagion, or the direct inoculation of the poisonous saliva by a bite. The term hydrophobia, which means fear of water, is applicable only to the disease as it is observed in man, since, as a rule, no such dread is manifested by the lower animals.

The causes which excite this disease in the members of the canine species are really unknown. Excessive heat, contrary to popular belief, can have no power to engender it, seeing that it is just as common in cold weather and latitudes as in warm.

The best description is contained in Youatt's work, from which the following extract is taken :

"The disease manifests itself under two forms. The furious form, characterized by augmented activity of the sensorial and locomotive systems, a disposition to bite, and a continued, peculiar bark. The animal becomes altered in habits and disposition, has an inclination to 
lick or carry inedible substances, is restless and snaps in the air, but is still obedient and attached. Soon there is loss of appetite and thirst, the mouth and tongue swollen ; the eyes red, dull, and half closed; the skin of the forehead wrinkled; the coat rough and staring; the gait unsteady and staggering; there is a periodic disposition to bite, the animal in approaching is often quiet and friendly, and then snaps; latterly, there is paralysis of the extremities; the breathing and deglutition become affected by spasms; the external surface irritable, and the sensorial functions increased in activity and perverted; convulsions may occur. These symptoms are paroxysmal, they remit and intermit, and are often excited by sight, hearing, or touch.

"The sullen form is characterized by shyness and depression, in which there is no disposition to bite, and no fear of fluids. The dog appears to be unusually quiet, is melancholic, and has depression of spirits: although he has no fear of water, he does not drink; he makes no attempt to bite, and seems haggard and suspicious, avoiding society, and refusing food. The breathing is labored and the bark is harsh, rough, and altered in tone; the mouth is open from the dropping of the jaw; the tongue protrudes, and the saliva is constantly flowing. The breathing soon becomes more difficult and laborious; there are tremors, and vomiting, and convulsions."

\section{IN OTHER ANIMALS.}

In horses, oxen, sheep, cats, pigs, the symptoms do not vary materially from those observed in dogs. We find, after the disease is developed, restlessness, difliculty 
of swallowing, modification of the voice in neighing, grunting, bellowing, etc., also biting, butting with the horns, scratching, etc., followed by paralysis and death.

\section{Treatment.}

From my own experience, I know of no remedy for this terrible disease. The best plan, probably, is to destroy every affected animal, in order to prevent the propagation of the disease. Youatt strongly advocates the free application of lunar caustic to the part bitten by a mad animal, as a preventive. A singular popular error is that a person bitten by a healthy dog will have this disease if the dog should afterwards contract it. Of course there is not a particle of foundation for such a notion.

\section{2.-Tetanus.}

\section{IN HORSES.}

Of all animals the horse is especially liable to tetanus - a disease of the upper part of the spinal marrow, characterized by unremitting spasm of all the muscles of the body. There are two forms: the idiopathic, which arises from exposure to severe weather, and irritation of the stornach and bowels; and the traumatic, which follows wounds and other injuries, such as broken knees, open joints, bruises, nicking or docking the tail, punctured wounds of the feet, castration, fractured bones, etc.

As a rule, the symptoms come on slowly. In the earliest stage, the muscles of the jaw and neck are the first to be attacked, and hence the convertible term of the disease-Lock-JAW. The animal manifests some 
difficulty in gathering his food with his lips, in swallowing, in moving his head and neck, and some degree of general stiffness in walking. Later, the muscles of the jaw are firmly contracted, hard to the touch on the cheek, and more or less completely close the mouth; so that by no force can the jaws be separated, nor can food by any means be nibbled up. The eyes are fixed, squinted outwards, pulled backwards into their sockets, and the "haw" drawn in front of the eyeballs. The neck is stiff and cannot be moved, and its muscles hard and rigid. The head is held firmly in one position, with the muzzle pointed forwards; the nostrils are expanded; the ears project forwards, erect and fixed; the lips are stretched firmly across the front of the teeth, exposing them to view; and slaver dribbles from the mouth.

The belly is tucked up, contracted, and hard to the touch; the tail is elevated and in a constant tremble; the anus is firmly contracted; the urine scanty and the bowels confined. The animal stands resolutely fixed to one spot, with all his legs stretched out; if perchance he move, or be made to move, he does so, not in detail, but all of a piece, like a thing without joint or suppleness, and the least effort evidently causes acute suffering. The breathing is quickened, short, and constrained; the pulse frequent and hard.

The spasmed muscles are screwed up to a still higher degree of agonizing tension by any excitement; such as loud noises, angry talking, and even by light and rustling of straw. When the animal is kept in a dark, quiet box to the care of an attendant who does his work with kindness and stealth, the spasms slacken somewhat, although the muscles are never wholly relieved 
from the tetanic grip until the disease is on the decline. In very acute cases, the animal appears to be com. pletely overwhelmed by the severity of the disease, and death occurs in a few hours; in others, death may not happen for several days.

\section{Treatment.}

The animal must be placed in a darkened, loose box, and be kept perfectly quiet and free from noise or excitement of any kind whatsoever. To protect it from cold, put on sufficient warm clothing, and bandage the legs with flannel. The man in attendance must do his work in the quietest manner possible, and keep his tongue still. Thin gruel, milk, linseed and hay tea, slightly warmed, should be frequently offered. As the jaws open, boiled turnips, bran mashes, and bruised oats are the best. After convalescence is fairly established, great care must be exercised to prevent overloading of the stomach.

The best remedies are the following:

Aconite and Belladonna are the best at the onset of the attack, when the disease can be traced to exposure to cold or damp. Give them in 20 drop doses every two hours alternately.

If there be no improvement in a few hours, I would recommend Aconite to be stopped, and Nux Vomica to be given in the same doses and times alternately with Belladonna.

Arnica is especially suitable when the disease is the result of wounds, and injuries in general; and in nine cases out of ten it will be necessary to give it in alternation with Belladonna, as directed above. 
In the fully developed stage, with intense rigidity and exacerbation of spasm on the least excitement, I would give Nux Vomica, alternately with Arnica, in traumatic cases.

Each dose must be mixed with a tablespoonful of . water, and this mixture injected into the mouth, by means of a syringe fitted with a long nozzle, which will admit of being insinuated between the upper surface of the tongue and roof of mouth, to the back part of the mouth. Or, having first cleared out the bowel by an injection of warm water, throw up 40 drops, or double the usual dose of the medicines above mentioned.

It is impossible to lay down more precise rules for the treatment of such a disease as tetanus, which requires careful individualizing in each case, such as a practitioner on the spot, and conversant with the specialties of the symptoms, can alone do.

In the only three cases that I have ever treated throughout since I adopted homøopathy, with the result of two recoveries, the medicines used were Arnica, Nux and Belladonna.

Encouraged by the testimony of observant veterinarians, and by the recovery of a man suffering from severe tetanus, treated by Dr. Moore, who has reported it in the "British Journal of Homoopathy," vol. xxiv., p. 506, I intend on the first opportunity to try Prussic Acid, 20 drops, 1st dilution, every two hours, in tetanus attacking the horse.

Some years ago, I proposed, on being called in in consultation with Mr. Williams, V. S., to use Chloroform as an injection. At the same time, homoopathic medicines were given, and, I understand, the case recovered. 
Wounds and injuries, if still unhealed, should be treated with Arnica Lotion. If the foot has been pricked in shoeing, the shoe must be removed, the horn pared away, and a poultice put on; adding some Arnica to the - poultice.

\section{3.-Hysteria.}

This name has been applied at my suggestion by Mr. Haycock, of Manchester, to a rare disease of the mare. The late Professor Dick, in his "Manual of Veterinary Science," mentions that he recognized three cases of it, also in mares. The pathology of the disease is quite unknown at the present time. The symptoms are not unlike those which are observed in cases of spinal apoplexy befalling the human subject.

The attack comes on suddenly, especially in mares that have rested and then worked hard, or have been lively and frisky at exercise. They begin to stagger, appear stiff and sluggish, are indisposed or unable to move on, and evidently wish to lie down. The hind legs appear to be partially paralyzed. I have seen them standing on their fetlocks, with the soles of the hoofs turned backwards and upwards. When got into the stable, they lie fully extended on the ground, covered with sweat. They are every now and then seized with violent spasm, roll violently about and strain strongly, when dark-colored urine is discharged; the eyeballs are full and projecting, the eyes red; the muscles of the belly and legs are strongly contracted, and as hard as a board to the touch. The pulse and respiration are considerably increased in frequency. Symptoms of com- 
plete paralysis of the hind legs appear, the animal makes desperate efforts to get up, but cannot, and soon sinks exhausted. In some mild cases, the severe symptoms abate, and the animal recovers. Such are the most important symptoms of this curious disease.

\section{Treatment.}

Begin with Aconite and Belladonna, in 20 drop doses, every half-hour or hour, alternately. If, after a few doses have been given, the animal is better, continue them every two or three hours, increasing the interval between the doses according to the improvement made.

If there is no change for the better, give Nux Vomica in the same manner, instead of the other two medicines; it is likewise required in recovering cases when the paralytic symptoms do not clear off rapidly.

Acidum Hydrocyanicum (prussic acid) would, I think, be a likely remedy of value in such cases, especially when the convulsive struggles are strong, and the muscles hard and firmly contracted.

In addition to the three cases mentioned by the late Professor Dick, I give my own experience.

\section{CASE I.}

In June, 1872, I treated a horse which had been under the care of two allopathic veterinarians for two weeks, and then abandoned for a week as hopeless, and pronounced incurable by several medical gentlemen, who were present when I was first called to see him.

Symptoms.-Pulse 110 ; respiration 65 ; the horse was 1 ying on his side, covered with cold sweat; had not been up in three wetks; muscles strongly contracted.

Treatment.-Gave Aconite and Bellacionna, 20 drops, every half hour, alternately, and in four hours he got up. ('ontinued this treatment for two days, then gave Nux Vomica, night and morning, 
and Aconite and Belladonna during the day. In five days he was well and went to pasture.

\section{CASE II.}

William Harter, of Niles, N. Y., had a sorrel mare, which had been sick three months, and during that time had been treated by three different allopathic veterinarians. The disease had gone to the right hind quarter, making her very lame; stifle very weak, and muscles and cords perisbed, leaving nothing but skin and bones.

Treatment.-I gave her Rush and Nux, three times a day. Also, rubbed the perished part with a salve made by taking two pounds of butter, two ounces of O,iganum, one-half ounce Tincture of Iodine, warming the butter and stirring well together, when it is fit for use. Under this treatment the mare was well in two months.

\section{CASE III.}

I also treated a mare belonging to Mr. Darrow, which, under allopathic treatment, had been left in the same condition as the one just mentioned. After being under my treatment for two months, she was well.

\section{Case IV.}

March 23, 1875, I treated another mare belonging to William Harter, of Niles, affected with the same disease.

Symptoms.-Pulse 90 ; respiration 65 ; muscles contracted and very hard; unable to get up, after the greatest effort.

Treatment.-I gave her Aconite and Belladonna, alternately, every half hour, 20 drop doses. On the second day, she got up, with help. She continued to improve during the day, and in a few hours was able to get up without help. On the third day gave $N u x$, three times a day. To all appearance she is now well.

\section{4.--Megrims.}

Megrims means the same as vertigo and giddiness, and depends upon a congested state of the brain. Although giddiness is a symptom of tumors and other lesions of 
the brain, "megrims" is generally restricted to that particular giddiness which never comes on except when the animal is at work in a collar, and which is due to pressure on the jugular veins. A tight or badly fitting collar is the direct cause, by impeding the return of blood from the brain along the jugular veins; and violent exertion, hot weather, dragging a heavy load up a hill, \&c., determine the attack. Some horses are so peculiarly shaped in the neck that they become giddy even with a well-fitting collar. Megrims from obstruction of a jugular left after bleeding with the fleam, is much rarer nowadays than formerly.

I have known saddle-horses seized with giddiness, spin round and round, and throw the rider off. Whether this arose from organic disease, or from temporary causes independent of all restraint affecting the neck, it is impossible to say.

The symptoms come on suddenly. The animal whilst going along suddenly stops, raises and shakes his head, looks wild with staring eyes, looks stupidly about him, and staggers or sways from side to side. If the collar be not at once drawn forwards towards the head, the animal reels and falls in a heap, or he springs forwards and falls heavily against any obstruction that may be in the way. He may now be convulsed more or less, the breathing being quickened and the nostrils dilated. Presently he gets up conscious, shakes himself, looks above him as if wondering what it all meant, and is soou himself again.

\section{Treatment.}

When the giddiness depends upon organic discases of the brain, the case must be regarded as incurable. When 
it arices from a too tight bearing-rein, or from an illfitting collar, the means of preventing an attack are obvinus enough. When an attack threatens, the collar should be speedily pulled forward, and cold water dashed on the head. If the animal be fat and full. blooded, the diet should be reduced.

Select, according to symptoms and general habit, from Aconitum, Amica, Belladonna, China. Conium, Lachesis, Nux Tomica, Opium, Pulsatilla, Pilıus, Siticea and Sulptiur.

1) OsE. - 20 drops of the selected remedy may be dissolved in a little water, and given once in one, two, four, or six bours, according to the urgency of the symptoms.

\section{Case.}

In October, 1850, I visited a valuable carriage horse, belonging to H. P. Ree, Esq., of Mancliester, which for four years had been the subject of megrims.

He is affected thus :- Whilst being driven he will stop with alarming suddenness-throw up his head-shake it wildly-turn round and round, the coachman having no control whatever orer his morements. Sometimes he will stand still for a minute or two, and then go on as if nothing had been amiss. The same phenomena are exhibited when the coachman rides him; he will hurriedly, and without a moment's warning, whirl round aud round, and will sometimts come down on his knees; then the attack being orer, he seems all right.

I gave 20 drops of Arnica, 1st dilution, night and morning.

This treatment was continued for a week, when, no attacks having come on, it was suspended.

Eight months afterwards he had another seizure ; the same medicine effected the same results as before.

He had, to my personal knowledge, no attack after this. Having become a roarer, he was sold seven years after my first visit, and then I lost sight of him. 


\section{5.-Sturdy, or Gid of Sheep.}

The disease thus commonly named is very frequent amongst sheep in some parts of the country, and depends upon the presence within the skull or in the brain, of the bladder form of the tape worm, derived from the dog. When dogs are fed with these worms from a sheep suffering from sturdy, the fully developed trenia is found more or less abundantly in their bowels within a few weeks afterwards; and when the joints of the same tænia are given to lambs, sturdy is developed in them. Consequently, whenever a shepherd's dog has tape-worm, the lambs and young sheep are certain to be the victims of sturdy.

At first, when the bladder is small, there are no symptoms of significance, but when it increases in size, and presses upon the brain, various symptoms make their appearance, varying with the position occupied by the hydatid, and the particular part of the brain upon which the pressure is exercised; and varying partly also owing to the soft brain of a young animal's skull yielding to the enlarging hydatid underneath. The sheep is dull, chews its food slowly and carelessly, and staggers when walking. From this latter symptom, the disease has received the name Staggers. When the bladder is situated in the substance of the brain, the sheep turns round and round with the head turned on one side, according as the right or left side of the brain is affeeted: and when located in the central line of the brain, the animal carries its head upwards and forwards. In some cases, it stands before a pool of water, apparently looking into it, and sometimes tumbles in and is 
drowned. In others, when quietly nibbling grass, it suddenly starts as if in a fright, and scampers over the field. If the bones of the skull should give away to the hydatid, the pressure on the brain is so much the less, and a sheep previously giddy and dull, becomes better for a while; or it may improve considerably, should the skull open and the fluid contents of the hydatid escape. As the hydatid is reaching a larger size, the giddiness and turning round are greater, more frequent, and more continuous. Paralysis and inability to stand up supervene. Other peculiarities are noticeable. When the eyes are involved, the pupils are dilated, the eyeball protruded and prominent, and vision more or less impaired - as we infer from the animal not going along with its companions, striking against trees or any other obstacle in its path, etc. When the hydatid is situated in the cerebellum, or small brain, the animal's rnovements are peculiar; it walks stiffly, leaps and falls, struggles and gets up, rolls about unsteadily on its legs, and so on. In all cases, whaterer the general or special symptoms may be, there is progressive wasting and weakness. Death is the usual termination, unless the hydatid be removed by a natural effort, or by an operation.

\section{Treatment.}

The only treatment that avails in this disease is to extirpate the hydatid after the manner of the Scotch shepherds, who feel for a soft part of the skull, pierce this part with a "borer," draw out the fluid in the bladder with a syringe, and sometimes drag out the bladder itself. I have seen them use a stocking wire. Such 
measures often succeed, especially when only one bladder exists, and the operation is not too long delayed. Dogs suffering from tape-worm should be banished from a sheep farm.

\section{6.-Apoplexy.}

Apoplexy of horses differs from staggers in this, that neither is there inflammation of the brain nor distension of the stomach.

The premonitory symptoms consist in the hanging down or resting of the head for support; evident dulness of sight and hearing, and a toppling, reeling motion, even when stationary; if erect, after a varied duration of these manifestations-(in a case within our recollection, they continued twenty-three hours) - a sudden fall takes place, muscular twitchings are observable, the vessels of the neck are distended, the muzzle is characterized by coldness, the eyes being prominent, wide open, and immovable, and the pupils much dilated, with grinding of the teeth, and incapability of deglutition; sometimes, expulsion of all fluids through the nostrils, and spontaneous evacuations; if convulsions ensue, the case is usually hopeless. Oxen, cows, \&c., are subject to apoplexy, exhibiting the following symptoms (with little or no premonition)-sudden falling, with loud, thick, heavy, noisy, or gurgling respiration, and fearful struggles. Sheep exhibit premonitory symptoms, such as slow and languid movements, apparent stupor, deficient: or lazy rumination, and slight heaving of the flanks; they then, of a sudden, become perfectly motionless as 
they stand; with insensibility of hearing; immovable and insensible eyes; dilated pupils; or even purple hue of the conjunctiva and interior of the nostrils (which are dilated); tottering of the limbs on attempting to move; thick, gurgling, or snoring respiration, and a full, hard (distensive), pulse; sometimes the pulse is impeded, confluent, slow, and full; lastly, the animal oscillates, and finally falls; the struggle which ensues is but of short duration. The pig betrays similar symptoms, but the eye has a more frenzied and bright red appearance, and the surface of the body generally appears to be numbed and insensible.

\section{Treatment.}

Select according to symptoms and general habit, from Aconitum, Antimonium crud., Arnica, Baryta carb., Belladonna, Cocculus, Conium, Digitalis, Hyoscyamus, Ipecacuanha (for sheep especially), Lachesis, Mercurius, Nux vomica, Opium, Pulsatilla, and Tartarus emeticus.

Doses.-On the first appearance of premonitory symptoms, administer repeated doses of the appropriate remedy, at intervals of from ten to sixty minutes, until the symptoms subside; or, immediately upon recovery from a paroxysm, administer a dose, repeating it after two hours, again after three more hours, and again after six hours.

\section{7.-Sunstroke-Coup de Soleil.}

Sunstroke may be called that form of apoplexy, in horses particularly, which is brought on by over-exertion and exposure to the intense heat of the sun. 


\section{8.-Staggers.}

Staggers is a disease to which horses are subject, and of which there are two varieties, viz., stomach or sleepy staggers, characterized by distension of the stomach, and purely occasioned by irregular and excessive feeding (eating too quick): and mad staggers with inflammation of the brain, and corresponding with that disease in other animals.

The symptoms of stomach staggers (with distension of the stomach) are as follows:-The animal stands listlessly, drooping, drowsy, and oscillating, or staring vacantly if disturbed; continually dozing, or dozing with food in the mouth, as, for instance, a mouthful of hay; and lastly, falling. Delirium sometimes ensues, the animal repeatedly falling, and getting up again, and violently striking at itself, or being seized with severe convulsions ; sometimes, also, there is great difficulty in backing; if in action, the feet are lifted very high; the head is turned right and left, without changing position, or the animal falling upon its haunches, and wheeling round, with the fore-legs stiff and straightened.

In MAD STAGgers the early symptoms are very analogous to those of the stomach or sleepy staggers; but as the real nature of this disease-inflammation of the brain-develops its characteristic features, violent heaving of the flanks ensues; the eyes become wild, red, and staring; the nostrils are strongly dilated; and, in the place of the heavy, drowsy, sleepiness of the eyes, the eyes become vivid, strongly and permanently opened, and furious delirium, with frantic movements, ensues; the animal rushes furiously from place to place, but with 
no malicious destructiveness, as in rabies, and evidently quite unconscious; wherens, in the rabid disease, consciousness is never lost; lastly the stupor returns in an aggravated degree, or the animil is perfectly exhausted and motionless. Colic is often characterized by movements and fury almost akin to that of staggers (frenzy, or brain fever), but consciousness is never lost in colic, and the rolling is the more prevalent and characteristic of the movements.

\section{9.-Inflammation of the Brain, Brain Fever, or Frenzy.}

This disease, which prevails amongst oxen, \&c., sheep, and other animals, chiefly at the height of summer, and when the heat is excessive, and which may at such times be provoked by over-driving, or excessive exposure to the direct rays of the sun, or by insufficiency of water, or excessive and over-stimulating food, is analogous to the mad staggers of the horse. Pigs are generally subject to this complaint, as the consecutive result of apoplexy.

Cattle, in addition to, or with some modification of the symptoms of mad staggers, also exhibit the following peculiarities in brain fever: During the frenzied period there is a peculiar aversion to, or excitation caused by, red (a color of which cattle have a natural abhorrence); the movements are, perhaps, more heedless and headlong than those of the horse; the tail is arched, the furious galloping incessant, and the bellowing frightful; the skin adheres to the ribs (as in hidebound), and the whole course of the spine and adjacent parts are peculiarly ten- 
der, on being touched; lastly, the animal falls head foremost, and either relapses into stupor or remains motionless from exhaustion ; the premonitory symptoms consist of vivid redness and prominence of the eyes; invincible repugnance to motion, heavy dulness and drowsiness, and thick, oppressed, and heavy respiration. Amongst sheep the symptoms partake of the features already described. It is more frequently developed amongst lambs than amongst full grown sheep. As in the case of the pig, it is liable to follow as the consecutive result of apoplexy.

Select from Aconitum, Arsenicum, Belladonna, Bryonia, Calcarea, Causticum, Cocculus, Digitalis, Dulcamara, Ignatia, Pulsatilla, Rhus, Veratrum (especially if the spine be severely affected); or, generally, Aconitum, Belladonna, Bryonia, Camphor Tincture (if caused by sunstroke), Cantharides, Cina, Cocculus, Cuprum a., Digitalis, Helleboris nig., Hyoscyamus, Lachesis, Mercurius, Opinm, and Sulphur.

Doses.-Of Opium Tincture, from 8 to 20 drops for oxen, dc., and from 4 to 12 drops for sheep and pigs, every half hour, until the symptoms subside. The administration of the other remedies should be such as directed in the following pages.

\section{Treatment.}

In giddiness, apoplexy, sunstroke, and mad and stomach staggers, or inflammation of the brain, 20 drops of the diluted remedy should be mixed in a little water for horses, a smaller number of drops for animals less in size, and given, as already directer, as soon as the premonitory symptoms set in, and the dose should be repeated at intervals of from ten to sixty minutes, until 
the symptoms subside. After recovering from a fit, the medicine should be given for a week at least, night and morning.

1 Aconite-Twenty drops mixed as above directed; to be given every hour or two hours, when the inflammation is very intense, the pulse rapid and hard, and the breathing labored. Aconite is our main reliance in the onset of inflammation, and should be continued till the violence of the fever abates, and the symptoms begin to indicate more particularly some other remedy.

Belladonna.-Twenty drops mixed as above directed, and a similar dose given every hour, or half hour, till easier, when the animal is plunging, rearing up, furious, unconscious. Attempts to bite; has a wild, fierce look; tries to leap out of the box; foams at the mouth ; trembles all over. Falls down, covered with sweat; remains lying a short time; rises again, and becomes violent as before. Belladonna is the most specific remedy for pure brain fever, especially when the disorder aggravates in paroxysms; and its influence shoud be continued as long as the improvement sustains its use. In this, as in all other remedies, the doses should be made farther apart as the symptoms become lighter, and the paroxysms return less frequently.

Opium.-Twenty drops mixed in a teaspoonful of water, every two hours, when there are redness about the eyes and eyelids; a dull, heavy, stupid look; drowsiness; his head hangs down, or he leans on the manger. Eyes glassy. Nostrils dilated. Obstinate constipation. Pulse slow and full. Suitable for sleepy staggers arising from an affection of the brain.

Arnica.-Twenty drops of the tincture in one pint of 
water; give a tablespoonful once in one hour, or, in extreme cases, once in half an hour, with external application of the Arnica lotion, when the disorder of the head or brain results from a blow, a concussion, or other external injury. The external application may be made in alternation, or at the same time with the internal exhibition of this remedy.

Gelseminum. - Twenty drops mixed in a little water; dose as in Opium, every hour, or two hours, in congestion of the brain from exposure to the heat of the sun, when there is a manifest, almost paralytic, weakness of the muscles and limbs; the pupils are dilated.

Glonoine.-A dose prepared as above directed for Gelseminum every hour, or two hours, in apoplexy, or sunstroke, when there is most violent congestion to the head. The eyes protrude, and have a wild, staring look, but there is not the fury described under Belladonna.

Nux Vomica.-Twenty drops, in a little water, may be given once in one, two, or three hours, according to the severity of the attack, especially of sleepy staggers, and where the patient has already been drugged, or labors under constipation, and in horses suffering from weakness or old age.

Stomach staggers-palsy of the stomach-is a disease sometimes temporary, caused by driving too soon after eating a hearty meal, especially of corn; and it may show itself in the mildest form by frequent and continual stumbling and hocking (stumbling with hind-feet) when driving under such circumstances. Nux vom. will greatly help in this case.

The proper treatment of stomach stargers (cansed in great measure by pressure of the contents of the stom- 
ach, flatus, and undigested food, upon the large nerves, and so reflected to the brain) will be greatly facilitated by freely opening the bowels as soon as possible. This may be done by giving one pint of Linseed oil, or half a pint of Castor oil, and, in urgent cases, the action of the purgative may be hastened by administering an injection consisting of half a gallon of warm milk and water, or warm soapsuds.

Stramonium.-Twenty drops mixed as above directed, and a similar dose once in three hours, may be given in what are popularly called " blind staggers," where the horse suddenly stops, shakes his head, staggers, falls down, presently gets up and proceeds on his way,-or he may stagger without falling Trembling and convulsion of the whole body. Rolling of the eyes. Nostrils fully dilated.

Lycopodium may be indicated in such a case as that described under Stramonium, and given in a similar dose, when the fan-like opening and closing of the nostrils is distinctly perceptible. It may then be given in conjunction with this remedy, a dose once in four hours. And in connection with Nux vomica when there is much and rapid formation of flatulence.

If no one of the above mentioned remedies seems to suit the case in hand, select, according to symptoms, from Arsenicum, Bryonia, Cocculus, Conium, Hyoscyamus, Lachesis, Anti. c., China, Rhus, Digitalis, Ipecacuanha, (for sheep especially,) Mercurius, and Tartar emetic.

To remove the tendency to apoplexy and many other brain diseases, Sulphur may be given twice a week with great advantage. 


\section{0.-Concussion of the Brain.}

This disorder, the result of some mechanical influence, needs no particular description. Every violent blow or fall upon the head is liable to result in concussion of the brain.

\section{Treatment.}

This is very simple. Arnica is the principal, and when the case is at all curative, the sufficient remedy. It should be given internally, in doses of six to ten drops of the dilution once in three hours, or three times a day, according to the severity of the original injury. Externally the Arnica Lotion should be freely applied thrice daily.

\section{1.-Water on the Brain.}

This disease gives rise amongst calves to symptoms very analogous to those of apoplexy; but the slow, protracted, often inactive, nature of these symptoms, besides the frequent enlargement of the head, will sufficiently distinguish it from an apoplectic affection, whose cowrse is always rapid. Lambs are subject to the same disease, which is of uterine origin. Many of the symptoms are also analogous to those of apoplexy, but here we further notice a more or less enlargement of the head, gradual and severe emaciation; sometimes an obstinate state of costiveness, and occasionally intractable relaxation; the appetite varying from total defieiency to craving; morbid, and ravenous consumption of food. 


\section{Treatment.}

Select from the following remedies: Aconitum, Arsenicum, Belladonna, Cina, Digitalis, Helleborus, Ledum, and Sulphur.

Doses. - From 4 to 20 drops, three times a day.

\section{2.-Hydatids of the Brain.}

A disease to which both sheep (especially) and cattle are known to be liable, and which, as regards sheep, is known by the familiar name of turnsick, and has also been designated by a variety of other local or rustic names. By whatever name it is called, it has been ascertained to arise from the presence of a quantity of small, peculiar insects which are lodged in various parts of the cavity of the head, either upon the division of the two portions of the brain, or within the substance, or upon the surface of the brain, or between the membranes which inclose it. Damp and ill-drained lands appear to provoke this disease, which is little known in elevated districts, or where the land has been efficiently drained.

The earlier symptoms are indolent and imperfect rumination ; irregular and insufficient browsing; listlessness, and solitary disposition ; oscillation during motion ; apparent absence of consciousness; the flesh wastes away; the expression becomes painful, and the face sunken; there is a peculiar attraction in the waterside, and if there be running water at hand, the animal will draw near to it, and stand abstractedly over it, often till giddiness supervenes, and it falls headlong into the stream or ditch; whilst browsing, the animal appears from time to time to be suddenly startled, and after star- 
ing stupidly about it for a moment, it suddenly starts away at the top of its speed; the substance of the eye becomes gradually discolored, until it is perfectly blue; and the head is fixedly turned to one and constantly to the same side; or sometimes on one side, sometimes on the other, or lowered forwards (with repeated headlong falling), and occasionally raised and reverted upwards towards the back, when the animal will oscillate from side to side in moving; these symptoms increase until the animal begins to spin round upon one spot until it falls, whilst, upon getting up again, the same motion is renewed.

\section{Treatment.}

Select from the following remedies: Belladonna, Cantharides, China, Cina, Graphites, Mercurius, Rhus, Ruta. Give ten drop doses. Upon the first detection of the premonitory symptoms, the administration should be repeated every twelve hours, until relief ensues.

Cows are subject to a similar affection, arising, also, out of the presence of this peculiar insect, inclosed in tumors generated by it, and forming upon the surface, between the membranes, or within the substance of the brain. The symptoms are very similar, differing only in the effects which are peculiar to the species; increased heat, more or less intense, in the ears and roots of the horn ; staring coat; dryness of the muzzle; accelerated and small (sometimes), or full and bounding pulse; little appetite, without rumination, and, by degrees, the commencement of the characteristic rotatory motion. The remedies above mentioned may be consulted, although the affection is obviously not very amenable to medication in any domestic animal. 


\section{3.-Paralysis. \\ IN HORSES AND OXEN.}

Paralysis means a total or partial loss of the power of feeling, or of moving, or of both, limited to one part of the body, or affecting the whole of it.

Facial Paralysis is confined to the muscles of the face, and is chiefly caused by pressure upon the nerves of the face by heavy head-gear, and by exposure to draughts of cold air. Usually only one side of the face is involved. sometimes both. The lip, especially the corner of the lower one, hangs down motionless, and appears to be swollen; the lips on the sound side are drawn towards that side, and the angle of the mouth drawn upwards. When the horse eats, he turns his head on one side-on the healthy side, so that he may use the unparalyzed side of his lips. The food is not cherved so well as usual, and it becomes crammed in between the teeth and cheek of the diseased side; and sometimes the morsel drops out. In some cases, the prick of a pin is not felt-showing paralysis of sensation as well as of motion.

Hemiplegia occurs when one side of the body is paralyzed. This rare form depends on effusion of blood or tumors on one side of the brain, or in the upper part of the spinal marrow. It comes on suddenly, like a "stroke." The animal falls down and cannot rise without help. The head is drawn to one side; the ear hangs down useless; the eye squints; a fore and hind leg of the same side are weak and cannot be voluntarily moved; and the animal either cannot walk at all, or he does so in an awkward, hobbling manner. 
Paraplegia consists of paralysis of the hinder half of the body, and depends upon disease of the spinal marrow, fractures of the vertebral bones, \&c. When the disease is fully developed, we observe that the animal is unable to stand, and tumbles down; he struggles to get up, raising himself on his fore legs, with his haunches remaining powerless on the ground, like a dog sitting. In this position, he may drag himself along the ground for a few paces. If he is raised on his feet, he cannot stand long, or at all, on his hind legs; the hind pasterns double under, with the soles of the hoof looking upwards. Unless recovery takes place, or he is destroyed, the symptoms of paralysis continue the same, the urine and freces escape involuntarily, and the hind legs mortify.

\section{Treatment.}

In all cases, treatment, in order to be successful, must be steadily continued for some time, as, even in the most favorable cases for recovery, improvement and complete restoration cannot be brought about speedily. Where the paralysis comes on suddenly from a severe injury, such as may be received in casting, from falls, from injuries to the spine in jumping, etc., the bone of the back may be broken, or the spinal marrow itself so much damaged as to preclude recovery.

The best medicines are the following:

Arnica is required when the paralysis follows injuries, blows, heavy bodies falling on the back, etc. Amica Lotion should be applied night and morning at least to the part.

Rhus Toxicodendron when the paralysis is the result of 
a sprain, or over-reach, as in jumping. Apply Rhus Lotion also.

After an injury is received, some degree of feverishness usually follows in a few hours; in such a case Aconite will prove useful.

Belladonna is required in those cases which have come on gradually, and are presumed to depend on congestion.

Nux Vomica, when there is reason to believe that the nervous centres are free from congestion, and that the paralysis is due merely to diminished nutrition of the spinal cord.

Graphites I have found to be the best for facial paralysis, and have cured many cases with it.

Doses._-20 drops for horses and cows; 10 for sheep and pigs. Give a dose three times a day.

\section{4.-Stringhalt.}

\section{IN HORSES.}

This name is given to a peculiar movement of the hind leg, arising from irregular, spasmodic action of the muscles, owing to some undiscovered disease of the nerves. Oliphant, in his "Law of Horses," says "it is probably so called from its resemblance to the sort of halt produced by a string tied to the leg of a pig, and held in the hand of the person driving it." Legally it constitutes unsoundness. It is incurable.

I have cured mild cases by the use of Cimicifuga and Nux Vomica, in 20 drop doses, six times a day alternately. 


\section{CHAPTER XIII. \\ DISEASES OF THE EYE.}

\section{1.-Ophthalmia.}

\section{IN HORSES.}

This is the name for inflammation of the mucous membrane which lines the inner surface of the eyelids, and is reflected over the front of the eyeball. The most frequent causes are, injuries from a stick or whip, or knocking the eye against a hard body; the irritation caused by a seed, a bit of hay or dust getting into the eye, or by the ingrowing of an eyelash; and an unhealthy condition of body, induced by living in a damp, ill-ventilated place. In catarrh, as has been stated in my remarks on that disease, the eye is more or less inflamed.

The eyelids are swollen and closed, and there is a copious flow of scalding tears, which run down the fice and fret the skin. At the corner of the eyes a small quantity of thickish mucus is observed at a later period. There is a great sensitiveness to light, and a strong reluctance to separate the lids, or have them separated by force. On examination, the membrane of the eye is 
seen to be red and traversed by a network of fine vessels, and the front part of the ball (cornea) is dim and muddy. In acute cases, there may be quickened pulse and other indications of feverishness; in chronic, there are none such.

\section{IN OTHER ANIMALS.}

Besides the general causes, one almost peculiar to oxen operates in the production of ophthalmia - it is inversion of the lower eyelid, whereby the lashes are turned inwards and keep up constant irritation of the part. This condition is called Trichiasis, by Dick.

\section{Treatment.}

It is necessary to examine the eye for foreign bodies, and, of course, to remove them, if found. The usual place for such bodies is under the upper lid, which should be turned inside out by taking the edge of it between the finger and thumb and turning it out on the point of the finger. In all cases, the eye should be bathed with warm water three or four times a day, light excluded, and no corn allowed for a few days. Much relief may be given, when a small portion of the cornea has been removed by a blow, or lash, by applying a drop or two of castor oil to the injured part. Whenever injuries have been the cause, Arnica Lotion should be dabbed on the outside several times a day.

Aconite is required when there are symptoms of feverishness.

Belladonna, when the eyes are very sensitive to light; the membrane of the eye red and injected; the tears copious; and the lids swollen and shut.

Mercurius Corrosivus is indicated, especially after, or 
alternately with the last medicine, when there is secretion of mucus, with sticking together of the lids, and when the cornea is hazy.

Euphrasia is sometimes of service when there is copious secretion and flow of tears, and great intolerance of light.

Nux Vornica may be required alone, or alternately with Belladonna or Mercurius, according to the indications for each, when there are symptoms of indigestion.

Sulphur is often valuable in chronic intractable cases.

Doses.-20 drops for horses and oxen; 10 for sheep and pigs. Repeat the doses every two or three hours, according to the violence of the symptous.

\section{2.-Periodic Ophthalmia.}

This disease, which is exclusively confined to the horse species, unlike simple conjunctiva, consists, not merely of inflammation of the superficial membrane covering the eye, but of inflammation of the entire eyeball - of all the structures enclosed within the globe. It is called periodic, from its relapsing or recurrent character ; sperific, from its presumed dependence upon some special, constitutional eause, of which no one knows anything; and moon-blindness, from its frequently occurring at the time of the moon's changes. None of these names correctly expresses what the disease really is.

Some horses are more susceptible to it than others; those, namely, of lax and flabby fibre, with flat feet and thick skin, and soft-hearted and funky at their work, especially if they have been bred and reared on damp, clayey soils, exposed to a humid atmosphere, fed on poor 
food, overworked, and kept in an ill-ventilated, illlighted, ill-drained stable. Pig-eyed horses, in whom the eye is small and sunk into the socket, are also peculiarly liable to suffer from this disease. It is beyond doubt that this affection is hereditary - that is to say, a sire or dam suffering from it, or blind from it, will transmit a tendency or predisposition to their progeny. It is more frequent in horses than in mares, and in young horses than old - both circumstances being explained by the local afflux of blood to the head during dentition, and the irritation of cutting the canine teeth, which are absent in the mare.

The symptoms begin either gradually with slight weeping and injection of the eyes, or suddenly, perhaps during the night, with swollen and nearly closed eyelids, and profuse discharge of tears. On making an examination, the animal shows his dislike to have the lids separated, and we discover that the eye is extremely sensitive to light, and pulled backwards into the socket, that the "haw" is red, swollen, and drawn partially in front of the eyeball, and that the conjunctiva is everywhere highly injected and red. At the same time, the pulse is full and frequent, the mouth hot and dry, the bowels costive, the urine scanty - all indicating a certain degree of feverish excitement, which varies with the suddenness of the attack and rapidity of its progress. In a few days, more or less, the turgid vessels are observed as minute red lines running into the rim of the transparent cornea, and the latter now presents a whitish appearance, either from being rendered opaque in itself, or from turbidity of the clear, internal humors, or from deposits of lymphy exudation. In favorable cases, the symptoms subside, 
often very quickly, the intolerance of light becomes less, the superficial redness disappears, the exuded matters are absorbed, and, in a first attack, the eye is restored to its healthy condition. In bad cases, the internal structures of the eye become permanently clouded, or utterly disorganized, and vision is lost forever.

The chief peculiarity of the disease is that the most favorable cases relapse, or subsequent attacks recur. The disease may fly about from one eye to the other, and its duration extend over several weeks, or even months each attack leaving the eye more and more damaged, until it exhausts itself in the utter destruction of the eye for all visual purposes.

Probably it would be found, if an examination were made by means of the ophthalmoscope as used in human practice, that even in the most trivial attack to superficial inspection, the delicate structures of the interior of the eye are left irretrievably damaged. To the view of the naked eye, the most notable changes are opacity of the crystalline lens, constituting CATARACT; specks on the cornea, or a diffused dimness; and a shrunken, pointed condition of the eyeball.

\section{Treatment.}

This is a most provoking disease to treat, for when a case is to all appearances doing well, a relapse takes place, and matters are as bad as ever, or even worse; and when one eye makes a tolerable escape, the sound one is attacked in its turn. Such a feature is, however, unavoidable, and as much an essential of the disease as for catarrh to be attended with a running nose. Under allopathic treatment, the uselessness of blecling, purges, 
fomentations, setons, blisters, eye-washes, etc., is admitted on all hands. As compared with it, homoeopathy, without pretending to save every eye, may confidently avow its superiority.

The animal should be placed in a darkened box, and light kept carefully excluded until the intolerance of light is notably less.

In the earliest period of the disease, when the animal is feverish, the pulse being quickened, the mouth dry and hot, etc.; and when the lids are almost shut and swollen, the membrane covering the eye injected, and the flow of tears copious, give Aconite and Belladonna, in turn, every two or three hours.

When the general feverishness is reduced, but the eye still remains inflamed superficially, and we notice a whitish, or brownish appearance in the interior, give Belladonna and Mercurius Corrosivus, in turn, every two or three hours, until the symptoms abate, when they may be given less frequently.

From the very outset and throughout the course of the disease, until the attack is fairly over, and the eye is resuming its natural clearness, it is of the first consequence to apply to the outside of the eye, and also, if possible, under the lids, Belladonna Lotion, which is made by dissolving 4 grains of the extract in one ounce of water. Pour some into the hollow of the hand and apply several times a day.

When the red and injected appearance of the eye is decidedly reduced, I would recommend Mercur. Cor. to be steadily continued for some time, three or four times a day, so long as any opacity of the cornea, or dimness of the internal humors remains. 
In relapsing, recurrent cases, attention should be paid to the ventilation and drainage of the stable, and the animal should be placed under a steady course of Arsenicum, 4 grains of the first trituration, three times a day; suspending it, and resuming the above medicines, should acute or sub-acute symptoms return.

I would likewise suggest a trial of Kali Bichromicum, and use one-half ounce of German wash in one-half pint of water.

Dos rs. -20 drops of the above medicine.

\section{3.-Cataract.}

\section{IN ALL ANIMALS.}

Cataract consists of opacity of the crystalline lens, or of its capsule, or of both. The lens is more frequently found opaque than the capsule. In health, both the lens and its investing membrane are perfectly transparent.The opacity may affect the whole or only a part of either of these structures.

The capsular variety of cataract is generally caused by inflammation, or by wounds or blows affecting the whole eyeball, or part of it. The opacity then comes on very quickly.

Opacity of the lens itself, although it may arise from the above causes, is usually formed in old animals as the consequence of senile degeneration of tissue from imperfect nutrition, and in horses as a very frequent consequence of "periodic ophthalmia."

The opacity that supervenes on inflammation is more 
likely to be removed than the form produced by old age, which always gets worse and worse.

Cataract may affect both eyes, or-only one. In old animals both eyes are usually cataractous, one wholly so, the other in part; whilst, if the opacity should have followed a blow or penetrating wound, the injured eye only is affected, and the other will remain sound until old age creeps on. As a rule, the blindness of old age depends on cataract.

Cataract is known by seeing behind the pupil an opaque body of a whitish grey color, which is best seen when the pupil is dilated by the previous application of Atropin. This preliminary step should always be taken in aid of correct diagnosis, when the case is doubtful.Of course, vision is more or less imperfect in proportion to the size and situation of the cataract. From the movements and behavior of the animal, the inferences may be drawn that vision is better in the evening or in a subdued light, than it is in the full sunshine; and that it is improved so long as the pupil remains dilated under the action of Atropin.

\section{Treatment.}

No medicine can remove cataract, as far as I know.And, in animals, an operation is practically useless.

\section{4.-Amaurosis.}

\section{IN ALL ANIMALS.}

Amaurosis, or gutta serena, is the name applied to a disease in which the optic nerve, or the brain, is so disordered, as to give rise to imperfect sight. "Amaurosis may 
be consequent upon some structural disease of the brain, or of the optic nerve. It may follow a blow, or some other form of violence received on the head. In some cases, it is difficult to make out the cause.

In gutta serena the eye is clear, bright, and transparent; the pupil is dilated, and the iris sluggish at first, immovable afterwards, as tested by the introduction of light into the eye. The movements of the animal show that there is partial or total blindness; he stumbles against every object in his way, and his whole gait is peculiar and characteristic. These symptoms may depend on several different pathological conditions of the interior of the eye. The human oculist has proved, by means of the ophthalmoscope, that the lesions which affect the internal structures are numerous and various, and that they are improperly classified under the general head of amaurosis. But such distinctions have yet to be drawn in veterinary pathology. The old names, though wanting in scientific accuracy, are retained as conveniently designating those cases of blindness which are dependent on obscure and unascertained changes in the visual apparatus, especially the nervous part of it.

\section{Treatment.}

One oz. of German wash in a pint of soft water, should be applied twice a day, and attention paid to the general health. 


\section{CHAPTER XIV. \\ DISEASES OF THE SKIN.}

Under such nondescript names as "surfeit," "mange," and so on, are conveniently clubbed together several entirely different skin diseases, incidental to the lower animals. These diseases can be classified, with at least some approach to diagnostic accuracy, by following the arrangement of human skin diseases into special groups.

\section{1.-Parasitic Diseases.}

Maggots IN Sheep.-The large blow-fly deposits eggs on the sheep, during hot days in summer, especially when the quarters and adjacent parts are fouled from diarrhoea. Any wounded surface is also a favorite place for such deposits. The eggs in process of time are developed into myriads of maggots, which eat into the skin, and cause deep sores, and free mattery discharge. The animal becomes depressed, eats nothing, and sinks from exhaustion, unless properly cared for and treated.

The treatment consists in clipping off the wool from the soiled or injured parts which the fly attacks; in keeping it thoroughly clean; in feeding liberally to support 
the strength; in giving 10 drops of Arsenicum, three times a day, or 10 drops of Carbolic Acid; and in dressing the part with Spirits of Tar.

WARBLES.-This name is applied to the swelling so often met with on the skin of oxen, occasionally on that of the horse, from the gad-fly depositing its eggs in the skin; a tumor, often as large as a pigeon's egg, containing grubs and matter, being the result. The back and loins are the favorite places for the fly's operations. There may be several such swellings. During the pro cess of depositing the eggs, the cow is in great fright, and scampers up and down the field in furious excitement.

The treatment consists in cutting into each tumor, squeezing out its contents, and afterwards applying Sulphurous Acid, three or four times a day; give 10 drops of Carbolic Acid, three times a day. The grubs should be burnt.

TICKS.-These blood-sucking creatures attack the skin of horses, cattle, and sheep; and more especially injure ewes and lambs in spring.

Dressing with olive oil, and subsequently washing with soft soap and water, are effectual and innocent measures. Use two ounces of German Wash in one quart of water. Give 10 drops of Carbolic Acid, three times a day.

LICE.-Various species of lice infest the skin of all the domesticated animals. Severe itching is set up, to 
relieve which the animal scratches himself until his skin is tender and sore. Filth and poverty are favorable for the development of lice.

There is a peculiar skin disease, named Phthiriasis, sometimes met with amongst horses where poultry are kept, from transmigration of a particular louse from the fowl to the horse. The itching is so excessive, that the animal is constantly rubbing himself, stamping the ground, kicking his belly, biting his skin, and altogether in a very sorry plight. In consequence of the scratching, the hair is rubbed off, and the denuded surface is covered with blood, or various kinds of eruption. This louse may visit the groom.

The treatment of lousiness consists in burning infected bedding and clothing; in washing harness and brushes in hot water ; in attending to thorough cleanliness; and in dressing every part of the hide with olive oil, or this failing, with Sulphurous Acid. The eruption caused by the lice either disappears of itself, after they are killed, or may be readily cured by giving the usual doses of Arsenicum, three times a day, or 10 drops of Carbolic Acid.

Scabies, Itch, Mange.-The disease bearing these names arises from an insect or mite which burrows in the skin, and induces severe irritation, followed by various eruptions.

Itch in the horse, according to the greatest authority Gerlach, is excited by three species of insects, namely the Sarcoptes Equi, which burrows in the skin; the Der. matodectes Equi, which bites and fastens itself to the skin ; and the Symbiotes Equi, which penetrates no further than 
the superficial layer of the skin. The first closely resembles the Sarcoptes of man, both in its appearance, and in the general features of the eruption to which it gives rise. It can live on man, and excite an eruption, which is identical with that of human itch, and which may disappear spontaneously. Grooms attending "mangy" horses have been known to suffer from horse itch, caused by the $S$. Equi. Cattle, also, are affected by it, but experiments have as yet failed in transmitting it to sheep and pigs. The second parasite is the special itch insect of the horse, as, if transmitted to the skin of other animals, it speedily dies. In the horse, it gives rise to itching, loss of hair, and a scurfy condition of the skin.The third is found in clusters, especially about the horse's heels; then the animal rubs one leg on the other, stamps with his feet, attempts to bite the part, \&c., and crusts of scurf form. It is also peculiar to the horse, and cannot live on other species.

In oxen, two parasites are found, corresponding with the two last kinds mentioned above as affecting the horse, but they do not live on the latter.

In sheep, the parasite fastens itself on the skin, and does not burrow at all-it is the Dermatodectes ovis, form. erly called the Sarcoptes ovis. The disease it gives rise to is called the Scab-one that is most injurious to the good condition of a flock, and difficult to eradicate, partly because it is communicated from diseased to healthy animals, less by actual contact than by rubbing against walls or banks to which the parasite, or its nits, adhere. This parasite is a most prolific creature.

In pigs, the parasite is a burrower, and can live on the human skin. 
The general symptoms of the itch disease are as follows :-When the parasite attaches itself to the skin, or burrows beneath the surface, irritation is set up, and pimples or vesicles appear. The extent of the eruption and the violence of the itching sensation vary according to the number of parasites present, and the thickness or delicacy of the skin. From the same causes, a general efflorescence appears in the vicinity of the papules, giving the skin a red appearance, which is best observed where the integument has little coloring matter in its structure.

Pustules are sometimes met with, and when they burst, the contained matter concretes into thick, brownish scales. This is especially the case in domestic favorites, with tender skins, and luxurious appetites. The pus. tules are chiefly located on the inner surface of the thighs, under the abdomen, in the pubic region, \&c. In these cases, the skin is moistened with a serous exudation, having the appearance of drops of sweat.

The common varieties of mange-red, dry, moistmark the different eruptive conditions of the skin. They all depend on the same cause-the itch parasite, but are immediately and chiefly produced by the vigorous scratching which the itching sensation provokes.Whether the eruption be a simple efflorescence, or papular, or vesicular, or pustular, will depend partly on the state of health of the animal, partly on the sensibility of the integument, and partly on the duration of the disease. Hence the external features of confirmed and de. veloped mange vary according to circumstances; but in all such cases, the hair drops off, the thickened skin- 
known to be thickened by pinching up a fold between the finger and the thumb-is thrown into wrinkles, especially about the head and on the back, and disfigured by scabs, chaps, and fissures. On some parts, small red points of clotted blood will be observed.

The itching is the most distressing symptom; slight at first, when the acari are few and the irritation caused by their presence in the skin is bearable, but gradually increasing with the rapid multiplication of the parasites. The itchy sensation is at first confined to the spots where they are deposited, and are engaged in channeling the epiderms; but, subsequently, the sensory nerves of the skin convey the sensation of universal itching, and the wretched animal spends his days and nights in rubbing, scratching, and nibbling himself.

In treatment, the object is to kill the parasites. First, clip off the hair or wool, and wash the whole body thoroughly with soft soap and warm water; then dry the skin; and, lastly, rub in Benzine. These applications may have to be repeated more than once; but one thorough application is generally sufficient, as far as the destruction of the parasites is concerned, and the remaining eruptions usually disappear without any treatment. Sulphur ointment is another good remedy; washing being used as directed. The whole body should be subjected to these processes at the same time, because if one parasite escapes destruction, others will soon be bred. Give 10 drops of Carbolic Acid, three times a day.

The bedding should be burnt. The stable furniture and clothing should be thoroughly cleansed with soft soap and hot water. 
Vegetable Parasites. - The production of skin diseases in animals, by low forms of vegetable life, has not yet received that investigation which the interest and importance of the subject demand. It is, however, beyond doubt that our domestic animals are sometimes attacked with RING-WORM, and that grooms attending on horses suffering from it have contracted the same disease. The eruption consists of a greater or less number of patches on different parts of the skin, circular in shape, partially or wholly bare of hair, and dotted with vesicles. Scales form on the surface of these patches, and on removing them there is a slight moisture underneath. The hairs around are altered from their natural color, and dusty.

There is a form of so-called "mange," which has been often observed where diseased straw has been used as bedding. A disease called "Camp Measles" has been observed in America in man, and has been ascribed to a fungus from diseased wheat straw.

The treatment consists in applying to the patches Sulphurous Acid Lotion, three times a day; in destroying infecting bedding; and in giving Arsenicum or Carbolic Acid, in the usual doses, three times a day. Liberal food should be allowed.

\section{2.-Erythematous Eruptions.}

The skin diseases of this class are characterized by slight redness of the skin, raised in patches, and irregularly circumscribed, the redness (when it can be seen) disappearing on pressure, and instantly returning on the removal of pressure. 
- ERythema. - This occurs from friction between folds of skin, such as between the thighs, in the arm-pits, \&c. The chafing of harness, also, causes it. Discharges running over the skin, as urine, may excite it. Hot weather, sweating, accumulations of dirt, favor this disease.

Another form arises mainly from pressure, as when horses are slung, and when saddles and collars gall the skin of the back and shoulders. Hence the name Saddle-gall. When the pressure is continued, the skin in the middle of the injured part becomes hard and gristly, and in some cases is separated from the surrounding healthy skin by an ulcerated furrow. This is Sitfast.

Cracked Heels also belong to this class. This very common disease of horses often arises from not properly washing and drying the heels, and especially if the horse be afterwards left in a draughty place. It is very apt to come on in frosty weather, when the heels are not thoroughly dried, and also when poor horses are suddenly put on a liberal diet. The symptoms are plain enough. The animal is lame and in pain. One or more of the heels is found painful, hot, and swollen. The skin cracks and fluid exudes. In bad or neglected cases, deep ulcerations form, and the legs swell.

Chapped Teats in cows or ewes is likewise erythema.

When the disease arises from friction and irritating discharges, the part must be thoroughly cleansed with tepid water and well dried, and then dusted with powdered starch, or fuller's earth. When the cause is pressure, the saddle or collar should be altered in such a way as to fit better, and Arnica Lotion applied frequently. In sitfast, the hardened skin may have to be cut out, in which case, the resulting wound should be dressed with 
Calendula Lction; if not, apply Arnica Lotion. For cracked heels, clip the hair close to the skin, foment if there is pain, poultice with bran if there is pain and dis charge, and if there is ulceration apply Sulphurous Acid, night and morning. At the same time give 20 drops of Arsenicum, or of Sulphur, or 10 drops of Carbolic Acid, three times a day; and feed on mashes, boiled oats, hay, and carrots. Chapped teats will readily heal after a few applications of Sulphurous Acid, with a camel's hair pencil. Heal with Butter Ointment.

URTICARIA, or nettle-rash, known as "surfeit," is very common in horses, less so in oxen and other animals. It'arises from indigestion, over-fatigue, and exposure to wet after a long journey. It is recognized by the sudden appearance of blotches, or elevations of the skin, varying in size from a sixpenny-piece to that of one's hand, on different parts of the body. There is considerable heat of the skin, and itching. In mild cases, the general health is not affected, and the eruption does not continue long; whereas in others, there is some amount of feverishness, and the elevations are prone to reappear at intervals for some time.

The treatment consists in giving mashes, but no corn for a few days, and in giving Aconite, Antimonium Crudum, Rhus, or Arsenicum - the first medicine for feverishness; the second, when the disease is associated with indigestion; the third when it is the result of cold; and the fourth, in obstinate or relapsing cases. Also, give 10 drops of Carbolic Acid, and bathe with German Wash.

Doses. - 20 drops for horses and oxen, every three or six hours. 


\section{3.-Papular Eruptions.}

Lichen.-Pimply eruptions are very common in horses. On stroking the skin with the point of the fingers, especially over the neck, shoulders, and hind quarters, a lot of hard, gritty bodies are felt. On scratching one of these pimples with the nail, we find that the top of it peels off as scurf. These pimples are as large as a hemp seed; generally break out in spring; are of long; duration and most difficult to cure. There is usually some itching and rubbing. Irritation and disorder of the stomach, drinking cold water whilst the body is heated, and sudden exposure to damp and cold, are the chief causes.

In treatment, Nux Vomica or Antimonium Crudum are required, when there are symptoms of indigestion.

Belladonna is required in those cases of papular eruptions which are attended with heat of skin, some feverishness, and great itching. Arsenicum proves of great service in all papular eruptions. Sulphur is a valuable remedy, and also, Carbolic Acid, in 10 drop doses.

Doses.-20 drops for horses and oxen; 10 for sheep-every four or six hours.

If there be reason to believe that parasites are the cause of the eruption and itching, dress with Sulphur Dintment, or Sulphurous Acid Lotion, night and morning. Attention must be paid to diet and exercise.

Prurigo. - This disease is signalized by small pimples, heat of skin, and particularly by excessive itching. The horse rubs his neck, root of the tail, mane, against 
the wall, edge of the manger, or anything else, until the skin is red, raw, and covered with small clots of blood. In some cases, the itching is intolerably severe, and the animal bites and rubs himself furiously. The legs are often mainly affected, and then he rubs one against the other, stamps impatiently, tries to nibble them, \&c.Many of these cases depend upon a plethoric condition of the system, the result of over feeding, and want of exercise. Others are connected with the presence of parasites, and others are dependent on an excitation of the nerves distributed to the skin. In this latter case, there may be no primary eruption whatever, and those that arise secondarily are wholly due to rubbing and biting. For treatment refer above to "Lichen."

\section{4.-Pustular Eruptions.}

ImpETIGO.-The chief variety of this class of skin disease occurs in horses, and is called "Grease." It consists of inflammation of the skin at the back surface of the fetlock and heels, followed by the formation of pustules, on the bursting of which there is a copious mattery discharge.

This is an inherited disease-often "runs in the family." Coarse bred horses and those with much hair on their legs are more subject to it than well bred horses, the difference being probably due to better grooming and attention in the one than in the other. Exposure to damp and cold, and dirt, are the exciting causes. One form of grease depends on a specific contagious fluid.

A swelling appears in one or more legs, the hind more 
frequently than the fore; this swelling may extend as high as the knees, or hocks. The skin is hot, red, and painful, and the animal is more or less stiff and lame in his movements. In a short time, clusters of small vesicles arise on the skin, at the heels, containing a clear fluid, which, if it be specific, has the property, when inoculated on oxen and human beings, of exciting an eruption like that of vaccine matter. If the fluid be not specific, it has no such property. The vesicles subsequently become pustules, which eontain matter. When these break, there is a more or less copious discharge of an offensive character. This discharge mats the hairs together, and dries into scabs. Still later, the skin cracks into deep fissures, from which a mattery discharge issues. The leg above the heel is much swollen and painful, and the cracks may extend upwards. The diseased surface, in the most advanced stage, becomes covered with large, unhealthy granulations, or "proud flesh," which, from their appearance, are known as "grapes." In the worst cases, what with the grapy condition of the leg, its considerable increase in size from swelling, the copious and offensive discharge, and the lameness present, the animal is in a sorry plight, and not pleasant to look at. In some cases, "canker" of the foot is present, as a consequence or complication, and in others of confirmed grease the parasites of the itch disease abound.

In the treatment of this disease, it is most important to keep the part perfectly clean by washing night and morning with luke-warm water and glycerine or petroleum soap, afterwards drying thoroughly with a soft cloth. If there be much offensive discharge and scabs, 
poultice with boiled carrots or turnips once or twice until the surface is clean. Mashes, carrots, and green food are useful as a change in the diet, and too much corn should be cut off. Arsenicum, 20 drops, three times a day, will, aided by the above measures, often arrest the disease in its early stage, or prevent it from going on to the ulcerated and grapy condition. Sulphurous Acid Lotion should be used thrice daily as soon as the skin cracks, and especially if you suspect the presence of the itch parasite. In some cases, I have used Arsenical Lotion with good effects. The following case shows good results from another remedy :

On April 2, 1860, I visited a pony belonging to Lady Frankland Russell, of Chequer's Court, Aylesbury. The pony, when bought two years and a half ago, had a slight running from the left hind heel. This has been gradually getting worse, in spite of the treatment of several veterinary surgeons, who have in vain applied the routine remedies. The disease is now above the fetlork joint, and involves the skin all around that part of the leg; the hairs stand up like the bristles of a bedgehog; an ichorous, offensive matter exudes freely : the pony is dead lame; the leg is much swollen.

Treatment.- The hair is to be cut short; the part affected to be poulticed for a day or two, and then the following liniment to be applied with a soft brush, night and morning - viz. : Kali Chloricum 1-2 oz., glycerine 12 oz. To have internally, Kali Chloricum, 20 drops night and morning. This treatment was pursued for a few weeks, when the leg was quite cured, the pony working all the time on dirty roads.

When I write, eighteen months after the above date, the disease had not returned; and no one can tell which leg was affected.*

WARTS.-One of the best local applications is strong tincture of Thuja, put on with a camel's hair pencil night

*Quoted from "Practical Reply," etc. 
and morning, and steadily persevered with. The best internal remedies are Thuja and Calcarea Carbonica - 20 drops for horses and oxen, night and morning; and Carbolic Acid, 10 drops.

MaLLenders is the common name for a scurfy or scaly disease (psoriasis) situate on the skin at the back part of the bend of the knee. Sallenders is the same at the front of the bend of the hock. Both attack horses only.

In treatment, after softening the scales with warm water and soap, apply Thuja night and morning, and give Arsenicum or Thuja, in 20 drop doses, at the same times. German Wash is an excellent external application. It is made as follows :-One quart of good strong cider vinegar, two ounces pulverized Blue vitriol, three ounces Alum, three tablespoonfuls pulverized Loaf sugar, six tablespoonfuls Honey, simmered together in earthen or pewter. 


\section{CHAPTER XV. \\ ERUPTIVE FEVERS.}

These diseases are attended with general fever, and a special eruption of the skin. They comprise, in the lower animals, erysipelas, measles, scarlatina, cow-pox, and small-pox.

\section{1.-Erysipelas.}

\section{IN ALL ANIMALS.}

Erysipelas occurs in all animals, but most frequently in sheep. In horses, it sometimes supervenes upon cracked heels. In sheep, it has been known to follow an injury of the skin inflicted during shearing.

The animal is more or less feverish - the pulse being quickened, the appetite impaired, the secretions checked, the mouth hot and dry, etc.

The skin is covered with a diffused redness, and is hot to the touch. The areolar tissue under the skin is likewise involved, giving rise to a swollen condition of the inflamed part. On the decline of the inflammation the skin peels off in thin scales. 


\section{Treatment.}

Belladonna and Rhus, given in alternation, are the best remedies, and in the majority of cases render the use of other medicines unnecessary.

Doses. - 20 drops for horses and cows; 10 for sheep and pigs; every four hours.

\section{2.-Measles.}

\section{. IN PIG AND SHEEP.}

By measles in pigs is meant, not the parasite disease, in which undeveloped tape-worms exist in the muscles, and give rise to what is known as "measly pork," but a fever, attended with catarrhal symptoms, and a rash on the skin. The animal coughs and vomits; the eyes are red and there is a copious flow of tears; and a watery discharge runs from the nose. The pulse is quickened; the skin hot and dry, and the appetite impaired. Then an eruption appears on different parts of the body, especially on the inner surface of the legs, about the armpits, etc., consisting of a multitude of small, red pimples. When the eruption fades, the inflamed skin peels off in small, branny scales. Diarrhœea, or bronchitis are apt to arise as complications.

A similar disease has been described by some continental veterinarians as occurring in sheep.

\section{Treatment.}

Aconite is required for quick pulse, hot, dry skin, thirst, restlessness, etc.

Pulsatilla should be given as soon as the rash appears, 
and when there is a hoarse cough, and running from the nose and eyes.

Arsenicum is required in those cases which manifest a tendency for the skin to slough in patches, and when diarrhœe, or prostration sets in.

If there are symptoms of bronchitis, treat as directed at page 165 .

D os es._-16, drops for sheep and pigs, every three hours.

\section{3.-Scarlatina.}

Horses sometimes present symptoms analogous to those of human scarlet fever. They become feverish, the pulse being 60 , or higher, and the breathing 20 , or upwards. The throat is sore, also some difficulty and pain in swallowing; the throat symptoms vary in severity in different cases. The glands about the throat are more or less painful, hot, and swollen, and there is a troublesome throat cough. If the nose be examined, we shall discover on the nasal membrane a number of scarlet spots of different sizes, which gradually run together and form red patches; a similar eruption is also found on the mucous membrane of the lips, and on the skin, wherever white hair enables one to see it. There is sloughing in the nose at the site of the spots, as is the case with the purple petechiæ of purpura hœmorrhagica. When the disease is on the decline, there is a copious shedding of scurfy scales. Blotches and elevations of the skin have been described by some writers, but they do not occur in genuine cases of scarlatina. After the disease has continued some days, and the horse appears to be doing well, a swelling, which pits on pressure, 
suddenly appears under the belly, gradually extending forwards to the breast and backwards to the prepuce, and also filling one or more legs. Recovery is the usual termination, unless " heroic" treatment is applied, or the disease attacks a worn-out animal.

\section{Treatment.}

In mild cases of the disease nothing more is required than to turn the horse into a loose box, feed him on mashes for a few days, and give 20 drops of Belladonna, every four hours. If the cough is troublesome, the throat sore and painful, and swollen, give the Belladonna alternately with the same dose of Mercurius, every two or three hours. When the fever runs high at the onset of the disease, it may be necessary to give Aconite, alone or alternately, with Belladonna. When swellings appear in the legs, and under the belly, and the urine is scanty, give Arsericum, 20 drops, four times a day.

\section{4.- Variola.}

\section{IN ALL ANIMALS.}

Variola is equivalent to small-pox in man. Horses, oxen, sheep, and pigs are subject to variolous diseases. Horses are affected in the lips, but especially in the heels, the attack in the latter region being undistinguishable in external features from common grease. If, however, the matter in the vesicle be inoculated on a cow's udder it will give rise to cow-pox, and if on a child's arm the well-known eruption, like that of vaccination.

In cows, the eruption appears on the udder, first as red, circumseribed, hard pimples, which gradually in- 
crease in size, until they become vesicles, filled with a clear fluid, and surrounded by a red ring. Later, the vesicles contain matter; they break, and the matter dries into scabs, which fall off. Some amount of fever is present. It is from these vesicles that vaccine lymph for vaccination is obtained.

In sheep, the disease is highly contagious, infectious and destructive to a flock. After a few days of incubation, during which the animal is dull and off its feed, small, red pimples appear, which grow larger and flat, and at first contain a clear fluid, subsequently matter. Then the pustule breaks, and the escaped fluid dries into a scab, grey or brown in color, which presently drops off. The attendant fever runs high. Discharge from the nose and eyes, hurried breathing, lividity of the membranes of the mouth and nose, and offensive smell of the skin, are frequently observed in ordinary cases. In malignant cases the vesicles contain bloody serum, and gas is developed under the skin; or death takes place suddenly before any rash appears ; or the air, or food tube is seriously implicated from the eruption appearing in one or other of them. The average duration is about three weeks. Sloughing of the skin in patches, or of the feet or hoofs, is a not unfrequent consequence.

\section{Treatment.}

The horse and cow-pox are so mild as to require no special treatment. When sheep-pox breaks out in a flock the diseased should be separated from the healthy, and the latter inoculated. They will then have the disease in a milder, more manageable, and less fatal form than if they had become affected by contagion, or infec- 
tion in the ordinary way. If it be resolved to place the diseased sheep under treatment, they should be carefully isolated during the illness, and for some time afterwards, great attention should be paid to ventilation and cleanliness, and only soft food and plenty of water should be given.

Aconite should be given when there is feverishness. Antimonium Tartaricum is indicated throughout the whole course of the disease, not only for the eruption, but for the lung symptoms so frequently present.

Arsenicum is useful for symptoms of prostration, for purging, and for tendency to sloughing.

Doses. -10 drops for sheep, every two hours. 


\section{CHAPTER XVI. \\ CARBUNCULAR DISEASES.}

The diseases of this class depend upon the development of a poisonous animal principle within the body of several of our domestic animals, and are characterized by the existence of a fever of a low or malignant type, and, in some forms, by a local lesion of the nature of carbuncle.

They all arise from rankness or poverty of pasturage, exposure to damp or cold, and from unascertained local causes. They attack herb-eating animals, and are communicated to others by contagion or inoculation, and have often prevailed as epizootics on the continent of Europe. They are most severe in full-blooded animals, and frequently follow a change from poor to rich diet.

True carbuncular diseases of animals are communicable to man by contact with diseased hide, horn, bones, blood, and flesh, and by flies and other insects carry= ing the poisonous matter from an infected animal's carcass to man. The disease is known in human medicine as "Malignant Pustule." The use of diseased flesh and milk as food does not appear to injure man, but the evidence on this point is rather conflicting. 
The following are the most common forms:

1. Gloss-anthrax, which the reader will find described at page 44 , as it occurs in oxen, sheep and pigs.

2. Splenic Apoplexy, which is described at page 112. Whilst some authorities regard this as a veritable carbuncular fever without external local lesions, others look upon it as non-specific congestion of the spleen.

3. Braxy in Sheep. - This is a very common and fatal disease amongst sheep, especially in mountainous districts, and in others, when the food is changed to turnips, etc., in winter. Amongst shepherds the term "braxy" is as nondescript as that of "the epidemic," "the distemper." Some mean by it an attack of diarrhoea, or of inflammation of the bowels, without any specific poison in the case. Properly speaking, it should be understood as designating a blood-disease, which suddenly attacks one or more sheep, particularly in frosty weather, and proves fatal in a few hours. The most usual symptoms are, in the early stage, staggering gait, tumbling forwards or backwards, hard, dry dung, and scanty, dark urine, with difficulty in passing both. The eyes are found to be red, the mouth hot and dry, the pulse quick and bounding, the breathing labored and panting, and the skin hot. Then the animal becomes weak, and drops down or rolls on its back, and soon dies. In some cases, before death, gas is generated under the skin, and a crackling noise is heard when the hand is passed over the back; in others, the paunch is distended with food and gas, giving rise to a swelling on the left side. After death, rapid decomposition sets in.

In treatment, very little can be done, except in the very earliest part of the attack. It has been recom- 
mended to cause the animal to move about briskly. It should be put into a warm place, and have hot gruel drenched down in small quantities. Warm water injections are useful when there appears to be irritation or pain in the belly, and the fæces are hard. When the paunch is much distended with gas, perforating it with the trocar may be necessary. In such cases give Nux Vomica and Ammonium Causticum in 20 drop doses, every hour, until there is improvement, and then every two or three hours. When symptoms of prostration appear, give Arsenicum, or Rhus, in the same way. Should recovery take place, great care as to the diet must be taken for some days. For the prevention of the disease, sheep should have the means of shelter on the hills, and should not be suddenly put on a rich pastureage.

4. Black Quarter. - This form is likewise called inflammatory fever, quarter-evil, joint-murrain, black-leg. It affects both oxen and sheep, and is widely spread in this and other countries. The young are more subject to it than the old.

When the disease is fully developed, there are symptoms of high febrile excitement, such as quick, full pulse, hurried breathing, outstretched head, bloodshot eyes, hot mouth, loss of appetite, moaning, anxious countenance, etc. The animal is lame on a fore or hind leg. There is, on some part of the body - on the quarters or about the joints - a painful swelling, which causes the animal to be dead lame, or very loth to move. The back and loins are exquisitely tender to the toich. Swellings appear about the back, shoulders, and loins, crackling when pressed upon, due to decomposition of 
the tissues and the generation of gas. The skin in patches becomes hard and dry, and subsequently sloughs off, leaving an ugly, unhealthy sore, which discharges offensive matter. At the same tinte, the mouth and tongue become ulcerated, and a stinking, bloody fluid drops from the nose and mouth. Diarrhœa sets in, and the animal dies from exhaustion.

This disease may be prevented by placing young stock in sheltered, comfortable quarters, carefully avoiding over-crowding, imperfect ventilation, and accumulations of excrement. Linseed cake, good hay, turnips, and salted water should be the diet.

The medical treatment has little chance of succeeding, chiefly in consequence of the virulence and great rapidity of the malady. Bleeding, and all varieties of severe measures, are worse than useless.

In my opinion, the following are the best remedies: Ammonium Causticum when the breathing is quick and heaving; the nostrils expanded; the muzzle dry, etc.

Aconite may prove useful when the pulse is full and bounding, and when symptoms of feverishness, as above detailed, are present.

Belladonna and Rhus, in alternation, as soon as the local swellings appear.

Mercurius and Belladonna, in alternation, when the mouth and tongue are severely ulcerated, and there is a copious discharge of saliva, or of bloody fluid.

Arsenicum is required for symptoms of prostration, and for diarrhœe.

The principal swelling should be fomented, and the sores which remain after the sloughs have fallen off, dressed with Sulphurous Acid three or four times a day. 
The same may be applied to the mouth and tongue, when ulcerated.

I use with good success Ammonium Causticum in 20 drop doses, alternately with Carbolic Acid, 10 drop doses, once in half hour, or hour, according to the urgency of the case.

D oses.-20 drops for oxen, 10 for sheep. Repeat each dose every one or two hours, according to the violence of the symptoms.

5.-Carbuncular Diseases in Pig.-One form is analogous to the gloss anthrax of cattle and sheep. The reader will find a description of it at page 44 . The second form is described at page 59. The third form, believed to belong to the anthrax family, is commonly known as the " blue disease," "distemper in pigs," "hog cholera," etc. It begins with dullness, loss of appetite, holding the head low; then the animal lies on its belly, and evidently suffers from pain there. Retching or vomiting of food, bile, or mucus is a common symptom. In some cases we observe drowsiness; in others delirium. The skin, especially about the back, belly, inside of the legs, is of a blue, or purplish color. Symptoms of paralysis of the hind legs appear; the surface becomes cold; and death may take place in a few hours.

In this disease, give Belladonna when the animal is excited, in pain from colic, and when the rash comes out. Rhus is also good when the rash appears and there are symptoms of prostration. Arsenicum for diarrhœa and exhaustion. For the paralysis, Rhus or Belladonna.

Doses. - 10 drops every one or two hours, according to the violence of the symptoms. 
The means of preventing these forms of disease amongst pigs comprise thorough cleanliness, removal of filth, clean bedding, sluicing the body with cold water, allowing exercise, and admitting fresh air. Sound food of both vegetable and animal nature should alone be given. When the disease breaks out in a lot, the animals should not be congregated together. 


\section{CHAPTER XVII. \\ WOUNDS AND INJURIES.}

\section{1.-Incised Wounds.}

Incised wounds are produced by cutting instruments, such as knives, scythes, etc.

In treating a wound, the first thing to do is to stop bleeding. This may be done by exposing the wound to the atmosphere, by pressure with the finger, or a compress of lint bound down by a bandage, by the applica. tion of cold water, etc. These means will always succeed, unless the hœmorrhage proceed from a wounded artery of considerable size. In such a case as this, firm and constant pressure is the immediate, and a ligature the radical remedy.

The second thing to do is, to remove all foreign bodies from the wound, such as clots of blood, dirt, splinters, thorns, by means of the fingers, or forceps, or affusion with water. No wound will heal whilst these substances remain in it.

In the third place, the sides of the wound should be brought together and kept there. The hair should be closely clipped away from the skin near the edges of the 
wound. Then, whilst the sides of the wound are held together, a thick layer of Collodion should be applied by means of a camel's hair pencil. This substance forms a thick film, under which the wound heals. But when the wound is larger or deeper, Collodion is not sufficient, and stitches may be necessary. Pass a strong needle, armed with silk or hempen thread, previously well waxed, through one side of the wound, from without inwards, then through the opposite point of the other side from within outwards. The thread is then to be tied, without unduly straining the parts, and the ends clipped closely off. Other stitches are to be put in in the same manner, at about the distance of half an inch, until the whole extent of the wound is accurately sewn up. A piece of lint, saturated with Arnica Lotion, and constantly kept moist, may then be placed over the wound and kept there by a bandage. In flesh wounds, with tearing and loss of substance, as well as a pure cut, apply Calendula Lotion. The stitches should be removed as soon as the sides of the wound are firmly adherent, and also when the wound becomes inflamed. In this latter event, poultices may have to be applied for a day or two, but usually Calendula Lotion and Aconite internally, in the usual doses for different animals, every three hours, will supersede the necessity for poulticing. Use also German wash or Butter Salve.

\section{2.-Broken Knees.}

Injuries to the knees consequent upon the horse falling upon this part, vary in extent and severity. For the 
purpose of illustration, they may be grouped into three classes :

1. Those which consist of simple bruises, without perforation of the skin. The knee is hot, painful, and swollen; some hair is removed and the skin somewhat grazed. 2. Those in which the skin is cut through, torn, and jagged, and the tissues underneath more or less injured. 3. Those in which the knee is cut, bruised, lacerated, and pulpified, and the knee-joint open into as well. This last accident is known by the escape of clear fluid, like white of egg. The injury is often so severe as to cause death, or to necessitate the destruction of the animal.

\section{Treatment.}

The treatrnent consists in the first class of cases, in washing the knee to remove dirt, and in frequently applying Arnica Lotion. In the second class, wash to remove dirt and blood, adjust the eut and torn skin as accurately as possible, and apply the same lotion. Give internally 20 drops of Arnica three times a day, and Aconite in the same way, if there be any feverishness. In severe injuries some inflammation will generally arise in the injured knee; the tissues ground down to a pulp by the force of the fall will slough off, and the wound will heal from the bottom, leaving, necessarily, a permanent blemish of greater or less extent. Here, hot fomentations and linseed poultices, medicated with Calendula Lotion, are required. When the inflammation in the wound is fairly gone, nothing more is required but this last lotion. When the knee-joint is open, treat as directed below. 
Front View of the Buncs of the Kinee.

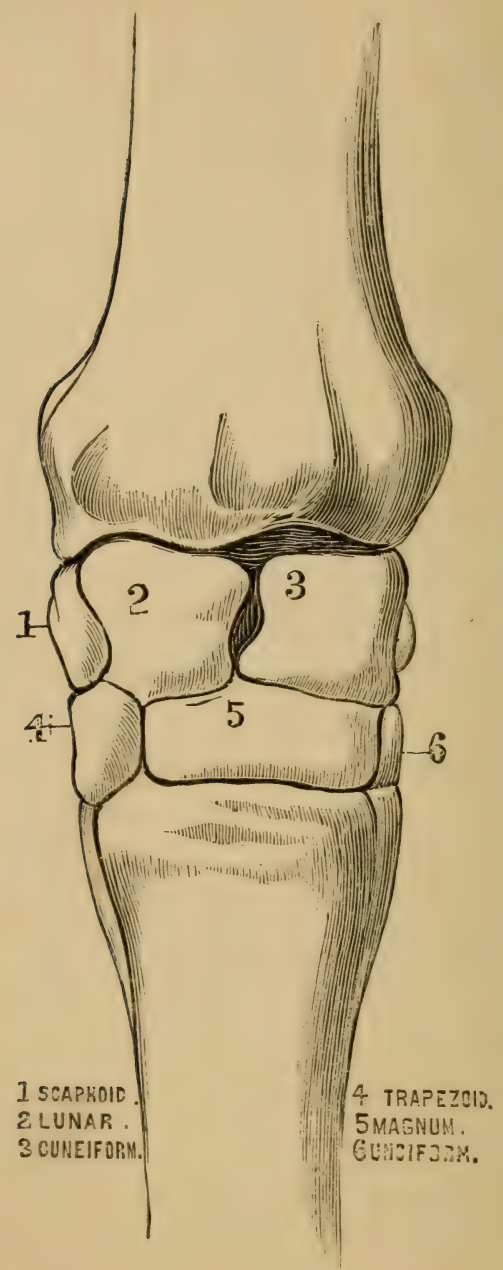

The tr $p$ zinm, or bone at the back of his knee, is $n$ t shown in tilis irawiag. 


\section{3.-Open Joint.}

This serious accident most frequently befalls the knee, hock, stifle, and pastern joints, and is caused by falls, kicks, stabs with a nail, or other penetrating body, etc.

It is characterized by a flow of synovia (joint-oil) through the wound. This fluid in appearance and feel is not unlike white of egg. Severe pain, and more or less irritative fever, varying with the size of the wound, and the importance of the joint injured, supervene. If, as sometimes happens, the interior of the joint becomes severely inflamed, the horse may die, or he may have to be destroyed; or tetanus may ensue.

\section{Treatment.}

In treatment, the first bar to recovery lies in the impossibility of keeping a horse quiet, and therefore, in keeping the joint perfectly motionless-rest being, in such cases, of incalculable aid; and the second lies in the condition of the wound itself, which cannot close and heal up, so long as a fluid is constantly running through its sides. When the wound is a large one, or when it implicates a large joint, such as the stifle, the horse must be slung. If there is much pain and feverishness, give 20 drops of Aconite, every three hours; if not, Arnica in the same way. When the flow of jointoil is considerable, give Silicea in the same doses. According to my experience, the best local application, one that is absolutely indispensable, is fresh slaked lime, very finely powdered. Lift some of it on the handle-end of a spoon, and apply it directly to the wound at the point where the fluid is escaping, and press it on with $13^{*}$ 
slight force. One attendant must wait on the horse during the day, another during the night, and whenever any oozing is seen, a fresh application of the powder must be made at the point of issue. The lime and the oil form a thick, hard, adhesive crust, which, in course of time, if assiduously added to, stops the running, and allows the wound to close. None of this crust should be removed until the discharge is completely arrested, but the fresh applications must be made on the top of the old. From a considerable experience of this treatment, I can strongly recommend it. Even very bad cases should not be given up. The great point is, the repeated applications night and day whenever the oil makes its appearance. Silicea should be continued from first to last; but alone it can do but little.

In opened knee-joint, it is possible to keep the leg in comparative rest, by fixing on a gutta percha splint, four inches broad and twelve long. Dip it in hot water to soften it ; then fit it to the inequalities of the back of the leg, and secure it by a bandage encircling the leg above and below the knee. Use German wash and butter salve, first one at night and the other in the morning.

\section{4.-Poll Evil.}

This disease, as the name implies, is situated at the juncture of the neck with the skull, which are connected together by a strong elastic ligament, commonly called the pack-wax. Poll-evil is at first merely a bruise, inflicted by the horse striking the top of his head against a door-way, or the top of a low-roofed stable; often, it is 
- 
1010500

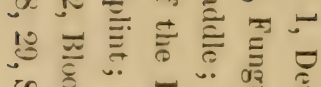

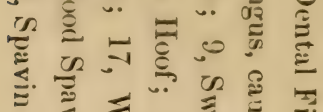

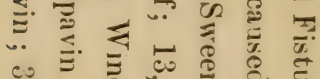

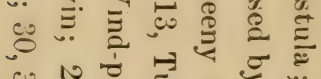

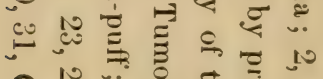

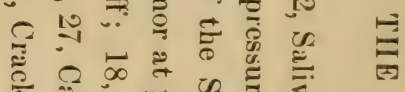

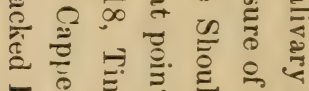

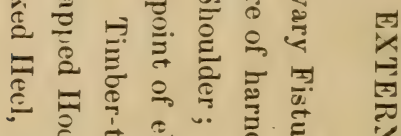

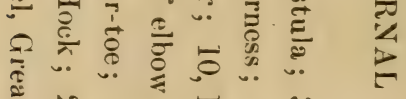

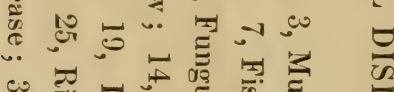

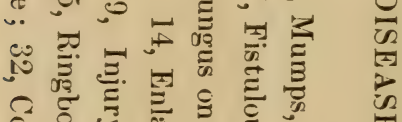

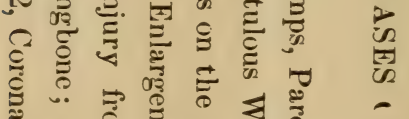

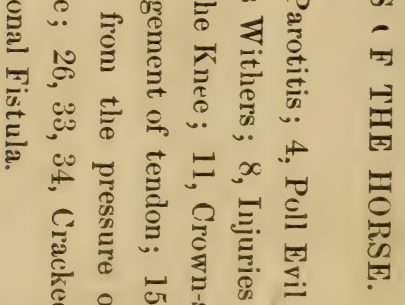

c 0 心

글. 인

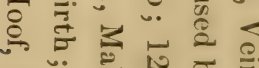

कृ

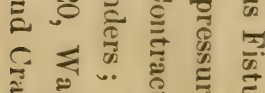

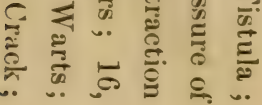




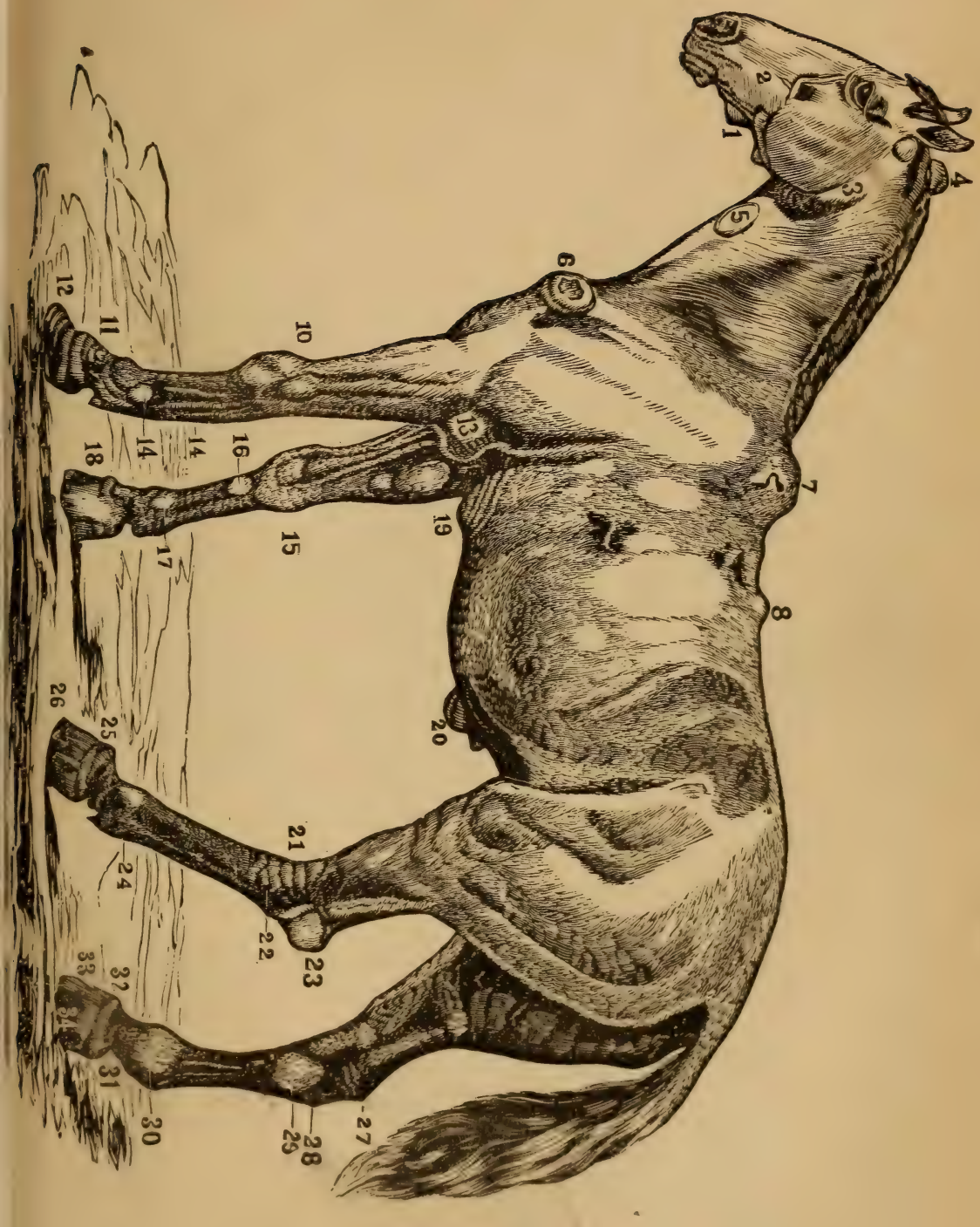



to be feared, the result of a brutal driver hitting that part with a stick, or the butt-end of a whip.

The seat of injury is very tender, hot, and swollen; the slightest touch causes the horse to flinch, and he is very reluctant to move it, and may be seen hanging his head, resting on the manger. If the case be neglected, the appetite fails, there is evidently greater pain, and the tissues under and about the pack-wax become inflamed. Matter soon forms, and, as there is no easy vent for it through such resisting structures to the skin, it burrows deeply and widely in all directions amongst the ligaments, and may even set up disease in the bones themselves. It may, eventually, if let alone, come to the surface and the abscess burst, to the animal's great relief.

\section{Treatment.}

The horse should rest, or, if this is impracticable, the head-collar should be eased in its bearings on the poll, and the driver should take care not to jerk the head about. Night and morning, the part should be thoroughly fomented with hot water. If these means should fail, it may be assumed that the formation of matter cannot be prevented, and the next step is to make a free incision into the abscess, so as to allow the matter to escape easily, and to make an independent opening at the lowest part. If the matter has already burrowed, the channels which it has made are called Fistula, or, in farriers' language, pipes. These also must be laid open with the knife. Then place dossils of lint in the lips of the wounds thus made, so that they may not close too soon. When matter is formed, and when it is discharging 
freely, give 20 drops of Hepar Sulphuris, four times a day ; and, in chronic cases, when it is desirable to quicken the healing process, give Silicea in the same way.

One quart of good, strong Cider Vinegar, two ounces pulverized Blue Vitriol, three ounces Alum, three tablespoonfuls pulverized Loaf Sugar, six tablepoonfuls Honey, simmered together in earthen or pewter, and applied with a syringe into the pipes or fistules of the Poll Evil, two or three times a day, is warranted to cure. Diseased bone should be scraped.

When the bones are diseased, the cure is always tedious.

\section{5.-Fistulous Withers.}

Beginning with a bruise from the pressure of an illfiting tsaddle, and ending in abscess and fistula, this injury presents exactly the same general symptoms as Poll Evil, runs the same course, and requires precisely the same treatment.

\section{6.-Bony Enlargements.}

NoDEs.-A kick, or other blow, on the shank bone of a hind or a fore leg, gives rise to severe pain, heat, and swelling. The membrane (periosteum) covering the bone inflames and thickens, and a deposit of osseous matter takes place. Constitutional causes, apart from any injury, may likewise induce such swellings.

Bone-Spavin.-This is a small, hard swelling caused by bony deposit, situated at the lower part of the hock, at the inner side. Usually, in the earlier stage at least, 
there is more or less occasional lameness, as well as tenderness to the touch, heat, and swelling. At a later period, it involves the hinge-like joint of the hock, and interferes with its free movement. In some chronic cases, the lameness attending spavin disappears during exercise, from a special part of the articulation being a ffected, whilst free movement remains between the tibia and astragalus. A peculiar make of hock, and hereditary predisposition favor the development of spavin; whilst tt is caused in other animals without any such liability; by overwork and excessive strain upon the joint, especially in early life.

Except in very confirmed cases, it is possible to remove spavin and its accompanying lameness without resorting to painful or disfiguring measures, by remedies mentioned below, which I have often tried and found successful.

SpLinT.-The bony tumor thus named is between the shank bone and the splint bone, which is situated alongside the shank bone, between the knee and fetlock, and it usually appears on the inside of a fore leg. Sometimes there is a splint on the outside. According to its position, size, and extent, it may, or may not cause lameness. If it be situated near the knee-joint, or iñterfere with the free movement of a sinew, it causes lameness. It then, and only then, constitutes legal unsoundness.

SIDE-BonE.-This consists of a deposit of bony matter into the side cartilages, at the back part and outside or inside of the coronet, just above the hoof. It is usually confined to the fore legs.

Ring-Bone.-This is a bony enlargement on the pas- 
tern bone, immediately above the coronet, extending in some cases so much as to produce anchylosis of the pastern, or coffin joints - a condition in which the opposed surfices of the articulation are surrounded and immoveably locked together by bony deposit. Ring-bone is most frequent on the hind legs, and there is usually " sidebone" as well. Ring-bone and side-bone, no matter how small, and whether they cause lameness or not, constitute unsqundness in the law's eye.

\section{Treatment.}

The treatment of all bony enlargements is the same. The frequent application of Arnica Lotion, and the internal administration of 20 drops of Arnica three times a day, is the proper treatment for such swellings, soon after the infliction of the injury. When the swelling is hard to the touch and bony in nature, give 20 drops of Mercurius Corrosivus, three times a day, and rub in, night and morning, Mercurius Corrosivus Lotion until the skin becomes tender and scurfy; then desist for two or three days, and repeat as before - continning in this manner until the enlargement disappears.

Refer to the appendix for recipes for the cure of Ringbone and Spavin.

The following cases, reprinted from "Practical Reply to Sir B. Brodie's Letter," illustrate two of the diseases described above:

\section{Case I.-Spavin.}

February 12th, 1861.--A horse belonging to Col. Dudley de Ros, is said to be lame in the fetlock joint. On examination I find lameness of right hind leg; slight enlargement at the spavin place of hock, with heat and pain on pressure. I ordered this part to be 
rubbed, night and morning, with the Merc. Cor. Lotion, and 20 drops of the sixth dilution of Merc. Cor. to be given night and morning. In a month he was sound, and went to work.

\section{Case II.-Incipient Ring-Bone.}

On July 14th, 1860, I visited, by the recommendation of Lady F. Russell, a carriage-mare belonging to Col. Ashley. She has been examined by Messrs. Mavor, who pronounce the lameness to arise from disease of the knee-joint, and recommend the mare to be sent to the farm, and have repeated blisters applied to the knęe. I found that the lameness was clearly due to incipient ring-bone, and the result of the treatment confirmed this diagnosis.

Symptoms.-Lame on the right fore leg; slight enlargement round pastern joint, with heat and pain on pressure.

Treatment.-A lotion of Merc. Cor. was applied night and morning, and Merc. Cor., sixth dilution, 20 drops, given twice daily; she was sound in a fortnight. Twelve months after I had a letter from Lady Frankland Russell, saying the mare remained quite sound.

\section{7.-Bursal Enlargements.}

A bursa is a small bag containing a fluid, and the use of it is to prevent friction where a tendon runs upon a bone. When a horse is overworked, too early worked, or the tendon is sprained, or the bursa itself directly injured by a blow, or kick, slight inflammation arises, the part becomes hot and tender, and a swelling, sometimes small, sometimes large, arises in consequence of increased secretion of mucus into the sac. There are several special varieties, including the following :-

Thorough-Pin, which is situated on both sides of the hock, in the form of a round swelling, often of considerable size, but seldom causing lameness.

WIND-GALLS, which are found above and at the sides 
of the fetlock joint. Except when large or hard, they do not cause stiff' action or lameness, but they are un. sightly and ought to be attended to.

Bog-Spavin is a bursal swelling, situated inside the bend of the hock, often of considerable size. This swelling, if large, obstructs the flow of blood from a vein which passes over the bursa - the vein is distended with blood-and the swelling thus caused is named BloodSpavin.

\section{Treatment.}

The treatment of all the foregoing injuries is substantially the same. At first, rub the part thoroughly, three times a day, with Arnica Lotion, and give 20 drops of Arnica, thrice daily. At a later period, when there is more or less copious effusion, use Rhus Lotion, and Rhus, in the same way. Should this fail, which it rarely does, or if the swelling be tense or callous, use Mercurius Corrosivus Lotion, and Merc. Cor. inwardly, as directed for "nodes," page 383 .

\section{- 8.-Capped Hocks, etc.}

CAPPED Hocks is a swelling on the point of the hock, best seen when looked at sideways, caused generally by the horse kicking in harness, or in the stable, or by any other mode of injury. It may be a true bursal enlargement; in which case the swelling is at the sides of the point of the hock; most frequently it consists of effusion of a serous fluid under the skin, with thickening of the skin itself. The swelling often becomes hard and diffcult to remove.

Capped Elbow.-This enlargement is of the same 
nature as capped hock, and is caused by the heel of the shoe injuring the point of the elbow when the horse lies down. Sometimes it ends in ABscess.

In treatment, rub in Arnica Lotion, three times a day, and give Arnica, 20 drops thrice daily. For subsequent hardening, use Mercurius Corrosivus Lotion, and Merc. Cor. thrice a day; also, German Wash or Butter Salve.

\section{9.-Sprains.}

A sprain may be defined to be an injury of the ligaments and adjacent structures of a joint, or of a single tendon, the result of over-extension. A sprain may vary in severity and consequences, from a slight strain upon these structures, requiring little or no treatment, up to a fatal or irremedial lesion. Local pain and swelling, with lameness or inability to move, and perhaps some degree of feverish excitement, are the chief general symptoms. The following are the principal individual accidents of this kind:-

SPRAIN of THE NeCK.-This occurs when a horse falls upon his head, as in hunting. There may be displacement of bones with twisting of the head and neck; or concussion or laceration of the spinal cord, attended or followed by hopeless paralysis; or simple sprain of the tendinous and ligamentous structures.

SPRAIN OF THE BACK occurs when a horse slips in the field, or on ice, and attempts to recover himself; or when the hind feet slip backwards, as in jumping. Severe injuries in this quarter may involve important parts and be beyond the reach of art; or the sprain may not be observable till the animal has rested. 
Sprain of the Shoulder occurs from a slip or fall, and is characterized by reluctance to move the limb, extension of the leg forward, dragging the toe along the ground and slightly swinging it round when walking, with local tenderness under pressure, and usually with some heat and swelling. When the lame leg is raised. well up, and pulled out in front of the horse in a straight line, it is evident that more or less pain is caused.

Sprain of the Hip is known by lameness, difficulty in moving the leg forwards, and local beat, tenderness, and swelling.

Sprain of the Stifle is known by dragging of the leg, and the same local symptoms as the last. The patella-or bone in front of the stifle joint-may be dislocated, in which case the leg is dragged along, stiff, and immovable. Drawing the leg forcibly forwards and pressing the bone into its place, will remedy this displacement. Spasm, or cramp of the leg, gives rise to similar symptoms; it comes and goes suddenly.

The Pelvis Bone, generally at the outer extremity, is frequently injured by strain or overwork. When this is the case, the foot on the affected side will be found resting on top of the other foot, the heel cork up to the hair on the foot.

Treatment.-Nux Vomica and Cimicifuga, alternately, 20 drop doses, each one four times a day.

CURB is an enlargement at the back of the hock, about three or four inches below the point of the hock, and consists of sprain, followed by swelling and thickening of the ligament which binds the os calcis and metatarsal bones together. It is seen at a glance, by looking at the hock from the side. A horse galloping over stiff ground, 

Tendons and Ligaments of the Fore-leg.

S. Splint bone

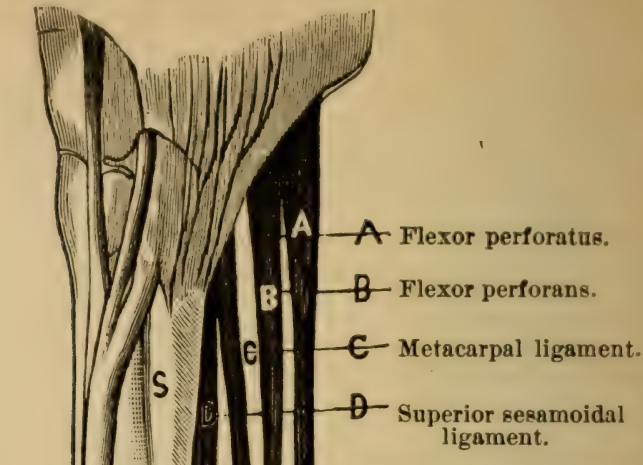

E. Extensor tendon.

M. Great metcarpal or cannon or shank bone

E. Extensor tendon.

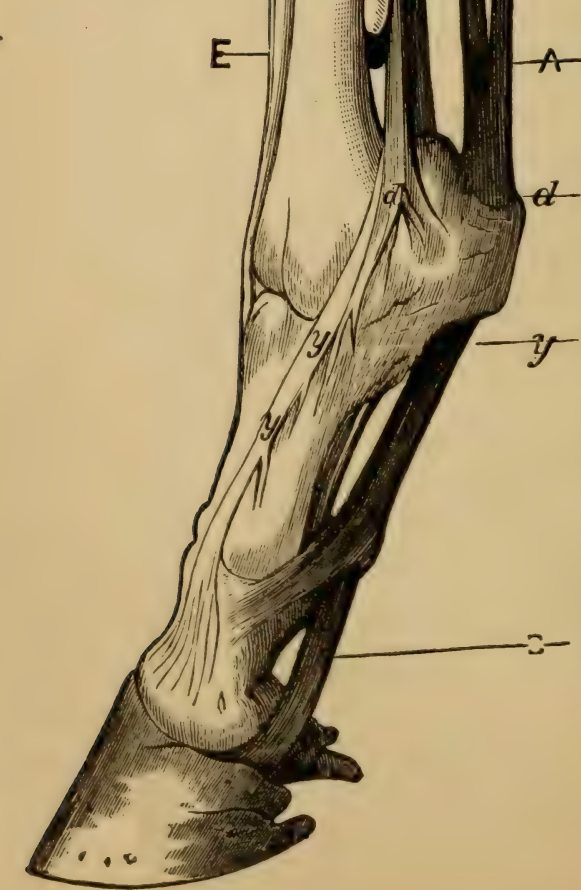

Continuation of the flexor perforans afterwards inserted into the lower side of the os pedis. 
or put to a sudden spring, as in a start or jump, may "throw out" a curb. Young horses are especially the subjects of it. Horses are called cow-hocked, or curbyhocked, when the joint is malformed in such a manner as to render them more than ordinarily liable to curb, from the ligament being kept constantly on the stretch. A horse with curb is unsound, but a curby-hocked horse not lame at the time of sale, is legally sound. A horse that throws out a curb even within an hour after sale, cannot be returned to the vendor.

Sprain of THE Back Tendons, a frequent accident to the fore leg, is attended with sudden lameness at the time of injury, or the lameness is perceptible only after the horse has rested. There is pain, heat, and swelling in some part of the tendon, between the pastern and knee. The horse rests his leg on the toe, and.is afraid to put the foot down flat. In bad, or neglected cases, the condition known as thickening of the back sinews, remains.

BRoken Down expresses rupture of the suspensory ligament, and of the perforating flexor of the foot-a serious accident which occurs suddenly to one leg, rarely to both, when the horse is galloping at full speed. He stops like a shot, or falls down-hence the name-and we find him resting on the fetlock, with the toe turned up and the sole of the foot forwards.

\section{Treatment.}

The treatment of all the above varieties of sprain is the same. They all demand absolute rest, at least until pain and swelling are markedly less, and the lameness trivial. Even then work is out of the question-gentle 
exercise is alone advisable. On this point, great care and judgment are required.

In the first stage of sprains, give Arnica, in 20 drop doses, every three, four, six, or eight hours, according to the severity of the injury, increasing the interval between the doses with the improvement. The best application is Arnica Lotion, which should be well rubbed into the injured part at least three times a day, or, when it can be done, applied constantly by wetting a piece of lint or cotton, covered with a dry cloth. In sprains of the leg, a bandage damped with the lotion, and kept damp, should be wrapped round. In the majority of cases this treatment will be successful. But if some swelling should still remain, substitute Rhus Lotion and Rhus, using the former and giving the latter as directed above for Arnica. In chronic thickening of tendons, or the sheaths of tendons, or of the structures around a joint, consequent on severe or neglected sprains, have recourse to Mercurius Corrosivus Lotion and Merc. Cor., as directed for "nodes" at page 292. Apply Butter Salve, also.

The following cases* illustrate the symptoms and treatment of sprains :-

\section{Case I.-Sprain of the Flexor Tendon.}

On May 5th, 1860 , I visited a carriage horse belonging to $\mathrm{B}$. Green, Esq. The animal is a fine topped horse, but rather defective in his fore legs. A few days ago he became lame of the fore right leg, and was sent to a veterinary surgeon, who recommended firing and blistering, and rest for three months. The owner objected to such severe measures, and placed his horse under my care.

Symptoms._Lameness of the right fore leg; the flexor tendon is

Quoted from "Practical Reply." 
swollen, hot, and painful, just above the fetlock joint; quite well otherwise.

Treatment. - Foment for half an bour, and afterwards rub in Rhus Lotion, night and morning; apply a bandage, wrung out of cold water, round the leg, and give 20 drops of Rhus, first dilution, night and morning. At the end of a week there was less lameness and swelling. Rhus Lotion was then used night and morning alone.Three weeks from the commencement of treatment, the horse was sound, the swelling subsided, and the leg as firm as the other.

\section{Case II.-Sprain of the Pasteern Joint.}

In 1860, I was consulted respecting a horse belonging to His Grace the Duke of —. I found great lameness, and swelling of the leg, from the effects of a blister which had been applied to the fetlock joint. I ordered the application of lard to soften the scabs, and after they had been washed off, A nica Lotion three times a day. Three weeks afterwards, when much improved, he was driven out, and returned worse than ever. There was heat and slight enlargement of the pastern joint, which I considered the original seat of the disease. My opinion was asked as to firing, and I said I could cure the case without. The horse was afterwards taken to a veterinary surgeon, who said the disease was in the fetlock joint, and who advised two or three courses of blistering, a dose of physic, rest, and firing as a last resort. As His Grace disliked such severe treatment, he consented to mine. I gave 20 drops of the sixth dilution of Merc. Cor., night and morning, and caused the pastern to be rubbed with the Merc. Cor. embrocation. In a fortnight the horse was sound.

\section{Case III.-Sprain of the Hock at the Seat of Spavin.}

On October 4th, 1860, I was requested to look at a bay horse, the property of the Midland Railway Company. This horse has been but a short time in the company's service, and is just recovered from a severe attack of bronchitis, and is not in a fit state for severe exertion. Last evening he was heavily loaded, and was driven at a quick pace. This morning he is very lame, particularly 
on the right leg; he walks very widely, and is stiff in his bocks; on the seat of spavin there are great heat and pain on pressure.

Treatment.- The hocks were ordered to be well fomented twice a day, and after each lomentation to be well rubbed with Ruta Lotion -two parts of the Ruta to fifteen of water. To have internally Ruta, 20 dinps, first dilution, night and morning.

This treatment was persevered with for a short time, when he became sound and fit to go to work.

\section{Case IV.-Sprain of the Hock. (Sprung Hock.)}

On the 17 th of October, 1860 , my attention was directed to a brown gelding, the property of the Midland Railway Company, which came home from work the previous evening very lame.

Symptoms. - The left hock is very much swollen all round, and projecting on each side; the seat of "thorough-pin," and also the seat of bog-spavin, are both very hot and painful on pressure ; otherwise the horse is well.

Treatment.-The hock to be well fomented three times a day, and immediately afterwards to be well rubbed with Ruta Lotion, and 20 drops of first dilution of Ruta to be administered internally.

Under this treatment the horse became sound in ten days, and went to work, although the hock was still considerably enlarged ; but by persevering in the above treatment, it became quite right in a few weeks.

\section{0.-Bruises of the Leg and Foot.}

Cutting and Brushing is the result of hitting a fetlock with the opposite foot. Some horses are so badly marle in the legs, that cutting is a matter of course.-The use of a boot, and putting on a particular shaped shoe, known to most smiths, are preventives.

SPEEDY.CUT occurs during the fast action, usually in horses with ill shaped legs, when the inside of the leg just below the knee is banged by the hoof or shoe of the 
opposite foot. A boot and special shoe may be required here also.

OVER-REACH is the consequence of hitting the heel of a fore foot with the toe of the hind one. If neglected, it may end in quittor.

TREAD is a bruise caused when one foot treads upon the coronet of another foot, as when the horse is turning or backing; or it may happen when one horse treads upon another. Sharpened shoes, in winter, often cause considerable injury in this way. Quittor may ensue.

\section{Treatment.}

In all the foregoing varieties of bruises, the horse should rest if possible. German Wash will generally cure any of them; but if it fails, Arnica Lotion should be applied three or four times a day, either by being put on with the hand, or by damping a bandage with it. Treads and over-reaches should be well fomented by placing the foot in a bucketful of water or in a bran poultice. After the fomentation, damp a piece of tow in Arnica Lotion, put it on the injured part, and fasten by a bandage. Keep the dressing constantly damp with the lotion, and renew the tow once a day. If there is a mattery discharge subsequently, put on a bran poultice medicated with a wineglassful of Calendula Lotion, and when the surface is clean, dress with this last lotion, and secure the pledget of tow by means of a bandage. Butter Salve is sometimes useful.

\section{1.-Quittor.}

Quittor is an abscess at the coronet resulting from injuries to the foot, such as corns, pricks in shoeing, or 
penetrating wounds produced by sharp bodies in general. In severe, or neglected cases, inflammation arises at the injured part, and matter forms, which spreads in every direction between the bone and horn, and at last appears at the coronet above the heel. The same condition of the coronet may follow severe treads, or over-reaches.Before the gathering breaks, we observe a painful swelling at the coronet; when this breaks of itself, there is a discharge of matter from one or more holes. The essence of quittor consists in there being sinuses, or pipes, communicating with the original seat of injury and the surface of the skin. Such cases are usually attended with considerable pain and lameness, and, unless promptly and properly treated, lead to disease of the bone, or deformity of the foot.

\section{Treatment.}

If the cause be corns, or penetrating wounds of the sole of the foot, the horn must be paired in such a manner as to let the pent-up matter out. Then put the foot in a pail of hot water, and afterwards in a large bran poultice. This should also be done for the swelling at the coronet, which should be opened if necessary. In bad cases the horse should not work. The best local application is Merc. Cor. Lotion, and German Wash, injected three times a day into the pipe at the opening on the coronet. If there is a second opening, stop it up during the injection, with the point of the finger, so that the fluid may flow along the windings of the sinus.

If the foot is pricked in shoeing, or if bad shoeing has caused corns, a skillful farrier will know what to do. 


\section{CHAPTER XVIII.}

\section{DISEASES NOT CLASSIFIED.}

\section{1.-Sweeny.}

This is an affection of the shoulder, and is caused by a wide collar, by influenza, or by inflammation on the lungs or the liver. When the inflammation is on the lungs, the left shoulder will be affected, and when it is on the liver, it will be in the right shoulder. I have also known it the result of contraction of the hoof. The cords and muscles being contracted, the shoulder perishes, and the animal becomes very lame. Sometimes sweeny is caused by tying the head to the foot, also by the animal jumping the fence fettered.

\section{Treatment.}

Use the liniment called Shoulder Medicine. In bad cases use the Salve. I have cured chronic cases of four years' standing, by alternating Salve one week with Liniment the next, and so on, alternately, until cured. When the foot contracts, soak it two days in warm water, using Oil of Tar one day, with fresh butter or Neats-foot Oil. Rub the foot well all over, and then shoe the animal with the Patent Horse Shoe.

Recipe for Liniment.-One pint Neats-foot Oil, one gill 
Hartshorn, half pint French Brandy, and half pint Spirits Turpentine.

Recipe for Salve for Sprains, Ring Bone and Sweeny.3 oz. Euphorbia ; 1 oz. Cantharides; $2 \frac{1}{2}$ drams Iodine; 3 drams Red Precipitate; 2 drams Bichloride Mercury; 1 oz. Mercurial Ointment; $\frac{1}{2}$ oz. Venice Tirpentine; $\frac{1}{2} l b$. White Pitch Turpentine; three-fourths 1b. Lard. Melt and mix the Lard and Turpentine, then add one ingredient at a time, as given in the recipe. Apply so much as will cover the enlargement, rubbing it in with the naked hand. Allow it to remain two days; then grease it; the third day wash, and then apply as before, and so continue until the enlargement disappears.

\section{2.-Glanders.}

This malignant disease is at once identified, in its incipient stage, by the comparatively clear watery appearance of the nasal discharge, as also characteristically by its peculiar adhesiveness. Sometimes it issues from one nostril only, sometimes from both, but it is always fluent - not accumulated and discharged in masses, from time to time, and with an effort. This discharge is sometimes barely noticeable, and may continue for very many months without farther progress in the development of disease, and without any apparent constitutional derangement. Yet, from the first, it is highly infectious, virulent, and malignant. By degrees there is an admixture of matter with the discharge.

At first, there is often no enlargement or swelling whatever in the glands, but subsequently, according as the discharge takes place from one or both nostrils, the 
glands (lymphatic, submaxillary glands) of the nether jaw will be affected on the one, or on both sides,-never, however, permanently, with general and diffused swelling of the adjacent parts, but with swelling of small kernels close to the bone; the membranes of the nostrils are characterized by a peculiar color, varying from greenish-blue to deep purple; and actual deep ulcers, with elevated margins - at first small, and gradually extending-will be developed upon the membrane of the nose (not as in cancerous ulcer of the muzzle slightly within, but yet on the extending part of the integument of the muzzle), the presence of which can be distinguished by the touch. Some symptoms of general disease are now developed, and as the discharge thickens with matter, becomes bloody and putrid, the strength, appetite and condition will rapidly fail ; the coat becomes staring, rough, and dull; the ulcers will multiply, the hair will fall off, the belly will be retracted, the respiration will be accompanied with a peculiar sound, as if the breath were expelled or inhaled through a multiplicity of little valves, and cough will supervene. (Compare "Strangles" and "Catarrh" for the distinctions ; in the latter stage of catarrh there is sometimes a stickiness in the discharge, which might mislead, unless all the attendant circumstances were duly considered.) Tubercular disease of the lungs is the last stage of glanders; and glanders and farey will ever be liable to combine, being very closely associated. It is, however, very essential to distinguish the first manifestations of glanders, lest it be spread not only throughout the whole of one team, but throughout many succeeding teams, through the excessive virulence of the infecting malignaney. 
This disease is seated in the lining membrane of the nostrils ; may supervene on catarrh, influenza, bronchitis, inflammation of the lungs, or strangles; more especially that form where the swelled gland does not suppurate, but becomes hard and cancerous. It would seem to be induced by bad ventilation, impure air, and impaired condition; but, more than all, infection is the chief source. If not arrested, it always proves fatal.

Early Stage.-Discharge, from one or both nostrils, of a sticky fluid, about the substance of white of egg, but grayish in color. Smell more or less offensive. The nasal membrane has a leaden hue, and soon becomes ulcerated. Submaxillary gland (generally one first) feels hard, enlarged, fixed. The nasal discharge may be confined to that side of the gland affected. There is no cough, unless complicated.

Second Stage.-The ulcers in the nostrils are generally visible, and often the discharge becomes thick, and smells very foul. There is general debility of the system; coat stares with a deaden look; mane and tail come off upon the slightest force.

\section{3.-Farcy.}

Farcy, which is frequently attendant upon confirmed glanders, or which is aggravated by the complication with glanders in the advanced stage, particularly when the vessels of the head are most distinctly affected, is externally, a very distinct disease-with the occasional manifestation of a variety of indications which charac- 
terize other diseases, such as eruptions like those of mange, apparently odematous swelling of the legs, affections of the foot and heel (cracking), wasting away of the flesh, as in phthisicky disorders; putrid discharge from the nose, as in glanders. The specific nature of the disease, however, consisting of the induction of a poison of a peculiar kind by the absorbent vessels, the characteristic features are: the development of little knotty tumors or protuberances, "farcy-buds," along the course of these vessels, and which are particularly developed about the numerous valves which occur in the course of such vessels, so that there is a general cordy distension of the vessel throughout, with occasional intervening lumps. The disease gradually advances; the buttons become more numerous, more prominent (being at first almost imperceptible), and hot, and generally excessively sensitive to the touch; ulceration (or occasionally induration) subsequently ensues. The development of these manifestations is sometimes preceded, but always followed, by rapid loss of appetite and condition, and by the staring, rough, unhealthy appearance of the skin, which characterizes most severe constitutional disorders. The muzzle, front part of the head, neck, and inner side of the thighs, are first (or alone) affected with the tumors; but sometimes there is a sudden unaccountable enlargement of the legs (or of one of the hind legs), the limb affected being almost incapacitated, and the symptom being accompanied with considerable constitutional disturbance, whereas but a few hours before there was no appearance of ailing. If the ulceration is unarrested, the tumors extend, become multiplied, the groin and fore-legs are affected, and ulceration follows the course 
of the absorbents in every direction; whereupon the appearance of glanders completes the deplorable and hopeless condition of the animal.

Glanders and Farcy are really modifications of the same constitutional disease. Inoculation with the matter of glanders or farey, or transfusion of the blood, may produce either disease in a sound animal. They result from the presence of a poison in the blood, attended with a peculiar specific inflammation. This poison appears to arise from degeneration of some of the constituents of the blood; but in farcy the virus seems to be in a less matured and less virulent stage than in glanders. Hence, farcy is in some degree amenable to treatment, whilst in glanders the disease nearly always proves intractable.

Farcy is often combined with glanders, but is a disease of the lymphatic system, its more common seat being the inner side of the thigh and leg, of the hinder extremity. It may attack the fore-legs, or the neck and lip. A small lump, named a farcy-bud, appears, varying in size; some, the size of a small nut, inflame, ulcerate, and open up a sore, which may extend to the size of a florin; from this broken surface a fluid exudes. At first these buds are confined to the leg, but soon extend to various parts of the body. When they approach the head and neck, glanders will also appear. Farcy is more readily cured than glanders, although inoculation of the same virus will produce either or both diseases. Between the farcy-buds, hard, cord-like swellings may be felt; these are the distended, inflamed lymphatic vessels. Farcy may be attended with dropsical swellings of the legs; and is then called Water Farcy. 
In some cases, a peculiar slipping, or cracking of the joints may be heard when the horse walks.

It is caused by mismanagement, excessive exhaustion, want of proper ventilation, want of drainage, impure air, neglected acute and inflammatory disorders, or anything which tends materially to deteriorate the vigor of the constitution; and, above all, contagion, that is, the infusion of the glanderous discharge upon any spot which may chance to be so excoriated (or otherwise) as to imbibe the poison.

\section{Treatment.}

The first and most important step is to remove the animal beyond the reach of others. Few can afford to assign a totally separate establishment to one useless animal, and therefore the next step would be to destroy it, lest infection should chance to be conveyed, by accident. But if there be means to devote some spot or building, or part of a building, to the glandered animal, the attempt may be made to cure the case; for Homøopathy alone holds out any prospect of saving animals infected with this poison. And the same means which might prove curative of a case actually developed would go far to prevent the development, if the suspicion is aroused of infection received, and they are employed in Homœopathy. At the same time every possible precaution should be taken to prevent the spread of this, the most virulent and destructive infection known to man. The least possible germ or effluvia from an animal suffering with this disease is capable of extending it to others, and thus the danger is again increased. The man who looks after a horse under suspicion of either of these diseases should not be allowed to go among other 
horses. The sponge, brush, pail, and other articles of stable equipment must, of course, be liept quite distinct. The attendant should be particularly warned that the disease is communicable to man, and no one with a broken skin should ever touch a glandered or farcied horse.

Mercurius, Arsenicum, and Kali hydriod, have been recommended for animals affected with glanders; but the ill success which has followed their employment hitkerto would not warrant our wasting time on these remedies, when there are others so much better capable of subduing this dangerous disorder, and of destroying its infection. The sovereign remedy for glanders and farcy is Kali Bichromicum and Carbolic Acid. Give ten drops in a little water, six times a day, for one day, of the Kali Bichromicum, and the same dose of Carbolic Acid, six times a day, on the second day, alternating daily the Kali Bichromicum and Carbolic Acid.

If there are farcy buds, wash them in a solution of Carbolic Acid,--one ounce of the acid to two quarts of water.

All sponges, brushes, and other articles employed about a glandered horse should be burned or deeply buried; as it would be more trouble and expense to disinfect them, even with this antiseptic, than they would be worth.

In addition to the specific remedies with which the disease must be antidoted, it is necessary to take every means to put the animal in good condition. As both glanders and farcy, when not the result of direct infection, come from overwork, poor fare, and damp, foul, and in other respects unhealthy, stables, so it is of the 
first importance to change all this. The diseased animal of course must remain at rest; his food should be nourishing and easy of digestion. Carrots and other green food and esculent roots are particularly recommended.

\section{4.-Rheumatism. IN HORSES.}

Acute Rheumatism is a febrile disease, attended with inflammation of the structures surrounding the joints, or of the lining membrane of the heart, or of the sac inclosing the heart. Some horses are more subject to it than others, owing to constitutional peculiarity, or hereditary predisposition. It is directly excited by exposure to damp and cold, either during health, or during convalescence from some catarrhal or pulmonary attack.

Sudden lameness is the first most marked symptom; it occurs in one or more legs, and in one or other joints, shoulder, stifle, hock, pastern, hip, etc. The affected joint, or joints, are hot and tender to the touch, and swollen. The animal stands still, and is very loth to stir a step. The breathing and pulse are both quickened-the latter also full and bounding. Judging from the animal's manner, there is severe pain in the part. The bowels are active, the tongue furred, and the saliva sour to the smell. There is a marked tendency to one joint being affected after the other, the disease often returning to the one first attacked. Above all, the heart and pleura are apt to be involved, in which case the gravity of the attack is greatly increased. In fatal cases, death from exhaustion, or from incurable lesion of the heart, comes on suddenly. 
Chronic Rheumatism is of the same nature as the acute, but the symptoms are much milder in character, and of longer duration. It is characterized by sudden lameness, of one leg to-day, of another to-morrow, especially after exposure; and there may, or may not be some swelling, heat, and pain in one of the joints of the leg. After cure, it is apt to reappear, and may continue more or less for months. It arises either as an independent affection, or as the result of an acute attack - most frequently the former.

Lumbago is rheumatism of the lumbar fascia, and an animal thus attacked is said to be "loin-bound." Pleurodynia exists when the disease affects the muscles between the ribs and the fibrous fascia lining the chest. The horse is said to be "shoulder-tied," or to have chestfounder, when the fibrous tissues about the shoulder are the seat of rheumatism. Lastly, cases are occasionally met with not unlike human sciatica.

\section{IN OTHER ANIMALS.}

Oxen are probably more subject to rheumatism than even horses. Cow leeches used to call rheumatism of the back, chine-felon, and of the joints, joint-felon. The symptoms, in their general features, of these different forms, or varieties of the disease, do not differ from those observed in the horse.

\section{Treatment.}

Aconitum is indicated at the beginning of the attack, when there are symptoms of febrile excitement; and also when the general symptoms and physical signs point out heart complication.

Belladonna, when the affected parts are extremely ten- 
der to the touch, and painful when moved, causing lameness. It is specially suitable when the shoulder is the seat of the disease.

Bryonia is indicated in cases of acute rheumatism, particularly of the legs, attended with frequent, full pulse, thirst, high-colored urine, pain aggravated by motion.

Nux Vomica is frequently beneficial when the stomach is out of order and the bowels costive, and in those cases which are attended with symptoms of paralytic weakness of the muscles of the legs.

Rhus is another remedy which is attended with good results in those cases where the animal appears to have lost the muscular power of the legs, and when the muscles of the back are principally affected. Also, when there is stiffness on first moving, and when the lameness is less after exercise.

Cimicifuga, Arnica, Arsenicum, Mercurius, and Sulphur, are also indicated in certain cases.

Doses.-20 drops for horses and oxen; 10 for sheep. Give a dose every two, four, six, or eight hours, according to the violence of the symptoms. Rub the diseased parts with Butter Salve.

The following cases, quoted from the British Journal of Hom. for 1858, are excellent examples of the symptoms and treatment of this disease in its different phases :

\section{Case I.-Rheumatism and Lumbago in Cow.}

On March 10th, 1858, I saw a cow belonging to R. Waller, Esq., Withington, near Manchester.

The symptoms are:-Pulse 72 and full; respiration right; great lameness of right leg; some swelling over the hip juint no 
appetite; hair standing on end; appears to suffer great pain; the back is stiff, and so tender that when pressed upon the cow groans aloud.

Treatment.-To have Aconite 1, 20 drops every three hours; keep in doors and clothe well.

Murch 11th.- She is now down and cannot rise; no other change. To have Aconite and Bryonia, 1st dil., 20 drops every three hours alternately.

Murch 12th.- Rather better; pulse reduced; has eaten hay and bran mash.

March 13th.-No further improvement. To have Belladonna 1, 20 drops, thrice daily.

March 14th.-Better; appetite improving; bowels getting right; has been attempting to rise.

March 15th.- She got up to-day; appetite good; rumination resumed. To have Sulphur night and morning for a few days.

\section{Case II.-Rheumatism-Heart Involved.}

A van-horse belonging to the London and North Western Railway Company. This horse is nine years old, and has had several rheumatic attacks during the four years he has been in the Company's possession. Twelve months ago, I attended him for an arthritic seizure, resembling the present one, only not so severe. Whilst out delivering parcels, and standing for a considerable time exposed to a cold, frosty, cutting, east wind, he was observed to limp at starting; this increased so much that he could go no longer, and had to be brought home.

November 24th, 1858.-Symptoms:-- Pulse 60 and full ; respiration 30 ; little or no appetite; dung hard; abdomen tucked up; urine scanty and high colored; he is in the stall, holding up the right hind leg about a foot from the ground; when made to move, he scarcely puts his toe to the floor; examined the foot and found nothing wrong there.

Treatment-To have Aconitum 1, 20 drops every three hours.

25tb.- Considerably better; still very lame, but can put the foot down ; pulse 64 and strong; respiration 24.

Continue medicine.

26th.- IJe is now as lame on the left hind leg as he was at first 
on the right; holds it up, and is evidently afraid to stand upon it; pulse 54, irregular and intermittent - sometimes strong and full, sometimes so weak that it can scarcely be felt; respiration quickened.

To have Digitalis 1, 20 drops every three hours.

27 th. - Much better; pulse 44, still intermitting occasionally; can walk on both legs alike; some stiffness when moved; fetlock joint somewhat swollen; dung and urine natural; appetite good.

Continue medicine.

28th.-Still improving; pulse 36 ; the horse is lively and eats well; rather lamer on the right leg again.

Continue medicine.

30 th.-Pulse is now regular; otherwise same as on 28 th.

To have Bryonia 1, 20 drops, thrice daily.

December 10th.-Gone to work.

\section{Case III.-Acute Rheumatism.}

March 16th, 1859.--A colt, belonging to Messrs. Syddall Brothers, Chadkirk, near Manchester, required my attendance.

Some time ago this animal was successfully treated for a severe rheumatic attack. On March 4th, he was found in the field early in the morning, having been turned out of his comfortable box by some mischievous fellow. On the following day he was feverish, off his food, stiff, and unable to move except with considerable pain and difficulty. Aconitum was given three times a day. On the 8th I was consulted, and prescribed Bryonia and Rhus; but no benefit following, my attendance was requested.

'I'he symptoms are:- Pulse 72, hard and contracted; respiration accelerated; nostrils dilated; the neck is held stiffly, the head being poked out; there is considerable difficulty in turning round in the box; in consequence of pain and rigidity of the.loins; the hind legs seem weak, particularly the right one; the lumbar region is somewhat arched and painful on one circumscribed part; the belly is tucked up; the horse walks in a peculiar manner, hesitatingly and weakly, as if from partial paralysis; he is irritable and easily excited; the appetite is impaired; the bowels regular; the urine scanty and high colored. 
Tiextment.-Tu have Rhododendron 1, 20 drop;, every three hours. 18th.-Better; pulse 54 ; walks better; neck less stiff; considerable swelling in the middle of abdomen; loins less painful, etc.

('ontinue medicine.

23d.-All right. The medicine to be continued night and morning for a few days.

24th.-Summoned to visit the horse, as he was much worse. Pulse 72 and full; respiration blowing and laborious; the skin is saturated with sweat; the left hind hock is very much swollen, and exquisitely painful to touch; the horse is dead lame, and can only point the toe of the affected limb; the mouth is hot and clammy; the urine turbid; the bowels constipated; the appetite gone.

To have Ruta 1, 20 drops every three hours; the hock to be well rubbed night and morning with Ruta Lotion, and a flannel bandage to be applied.

25th.-Received a message to the effect that the colt was considerably better.

27 th. - No lameness; the hock is still somewhat swollen, also the fetlock joint ; eats well and is hearty. Continue medicine and lotion.

30th.-Well.

\section{5.-Myalgia.}

In this disorder the muscles are the seat of pain. Horses, more frequently than other animals, are affected with it after racing, hunting, or any other severe, or longcontinued exertion. Soreness and stiffness express the same state in man. Usually, the pulse and breathing are little, if at all, disturbed, except after exertion, or when there is considerable pain. When the muscles of the chest and shoulder are principally affected, the disease resembles rheumatic pleurodynia, or spraining the shoulder, in which latter ease the horse walks down hill 
with great pain and difficulty, but up hill with comparative ease. Pressure of the affected muscles shows that there is great tenderness. Myalgia may be confounded with chronic rheumatism, from which, however, it chiefly differs in not attacking fibrous structures, or those entering into the formation of joints - in usually following severe exertion, or from a horse being overweighted - and in the shifting character of rheumatism being absent.

The best remedy is Arnica-20 drops every four hours.

The following was probably an instance of myalgia:

\section{Case.}

On June 3d, 1858, a pony, 13 hands high, belonging to Mr. Kershaw, solicitor, Manchester, had the following symptoms: Back arched, and as stiff and immoveable as if the vertebræ were made of one piece; difficulty in moving forward both legs, especially the right one; evident pain attending motion; soreness of the muscles; lies down much; when crouching, preparatory to lying on the ground, considerable hesitation is manifested, arising no doubt from the pain and difficulty attending the muscular movements; instead of lying down slowly and gently, he drops to the ground; he rises with great difficulty; the appetite is tolerably good; the pulse 44 , and the breathing 20 per minute, etc.

Treatment.-To have 20 drops of Merc. v. 5, and the same dose of Rhus 1, every six hours; rub Rhus Lotion on the loins and shoulders.

On June 4th, pulse and breathing normal ; back in natural position; much improved in all other respects.

On June 5 th, the groom called to say that my patient was prancing about at his exercise this morning. 


\section{6.-Purpura Hemorrhagica.}

\section{IN HORSES.}

This disease depends upon some unascertained alteration in the blood, or blood-vessels, or both, which allows the blood to escape into the structure of the skin, into the tissues under the skin, and into internal organs, and cavities, and ducts.

The causes are believed to be those which are calculated to lower vital power, or to impair the process of blood-making (sanguification), such as close, ill-ventilated, damp stables; overwork; bad or scanty food, etc. I have noticed that in nearly, if not in every case, the symptoms of purpura have been preceded, at a longer or shorter period, by a catarrhal affection.

The pulse ranges about 60 , and is full ; the breathing about 20 per minute. Swellings of variable size and irregular outline, and consisting of effused blood, suddenly appear under, or in the skin, in different parts of the body-especially the legs. The swelling of the legs about the hocks and knees is often enormous, and ends abruptly at the stifle and elbow joints. The lips and nostrils are much swollen, tense, and shining. The openings of the nose are often so much narrowed as to cause some difficulty of breathing. On examining the nasal membrane, it is found very red, and studded with purple spots which vary much in size-from a shilling downwards.

The inside of the lips is likewise covered with similar spots. If one of them be pricked, fluid blood exudes.

Usually, in a day or two, the pulse becomes weaker, the urine high colored, and further extravasations occur, 
unless the attack :akes a favorable turn. The swellings, which at first have a well-defined margin, which is gradually lost in the adjacent skin, extend to the breast, flank, belly, quarter, \&c. ; the membrane of the eye is blood-shot; a dark, bloody, fluid, alone or mixed with matter, flows from the nose; the nasal membrane becomes black, and the former spots slough off, and the tissue is seen hanging in shreds. The patches of swelling on some parts of the body become cold, hard as a board, and insensible to feeling-die, in fact, and shortly slough off, leaving raw, unhealthy sores. Other patches, especially those under the belly, exude a fluid, which may be seen hanging in drops on the hair. There is also in severe cases a discharge of blood by the urine, either mixed up with the urine, or passed in small clots. In addition, the animal is weak, eats little or nothing, and cannot move his legs, from the swelling preventing bending of the joints.

Such are the essential symptoms of this disease.

\section{Treatment.}

I have tried several medicines, but now I only use Kali Bichromicum alternately with Carbolic Acid. These I regard as specific, from their remarkable power over this disease.

During the last seven years, I have treated eighteen cases, with only one death, ten of them in horses belonging to the Midland Railway Company.

The following are cases in point:

\section{Case I.}

On the $23 \mathrm{~d}$ of October, 1860 , I was requested to look at a bay horse, the property of the Midland Railway Company. This horse 
had a severe attack of bronchitis on the 16th of September, and resumed work on the lst instant, since which time he has been well and improring in condition, until yesterday, when he was observed by the horse-keeper to be off his food, and looking dull and heavy.

Symptoms.-Pulse 60, full and strong ; respiration 20 ; fore legs, particularly the left one, swollen up to the knees, or rather higher, and in conseqnen e of this swelling he moves with very great difficulty; the Schneiderian membrane is beautifully spotted with small purple spists about the size of a pin's head.

Treatment. - To have Aconite, 20 drops, first dilution, every three hours.

24th.-Much better; pulse 44, and of the usual character ; respiration 14; the swelling is now up to the chest, but decreasing a little on the legs ; there is a considerable and painful swelling on the belly, near the left flank; the purple spots on the Schneiderian membrane are much fainter ; appetite good ; to have Kali Bichrom., 20 drops, first dilution, every three hours.

25th.-The swelling on the right leg is nearly gone, and that on the left is somewhat dıminished; also that on the belly; appetite good; pulse and respiration same as yesterday. Continue medicine.

26th.-Considerable amendment; pulse 40 ; respiration 10 ; swelling of the right leg entirely gone; that on the left is considerably diminished, an 1 the swelling on the belly is gradually disappearing. Continue medicine three times a day.

28 th. - Pulse and respiration normal ; there is a little swelling on the left knee, and on the side of the abdomen; otherwise the horse is weli. Continue medicine night and morning.

30th. - Well, and gone to work.

\section{CASE II.}

On August 13, 1861, Mr. W_ c ıme for me to go and see one of his horses which was unwell. The horse has been ill for four days, but had previously cold and cough before the swelling came on.

Symptoms.-Pulse 6t, and full ; respiration 14 per minute; the nasal membranes are thickly studded with scarlet spots, from the 
size of a pin's head to that of a shilling, and the alæ are somewhat swollen; the hind legs are swollen up to the patella, where the swelling terminates abruptly; the sheath is also enormously swollen, and so are the fore legs up to the chest, where the swelling terminates abruptly; the urine is scanty, and high colored; fæces contain more mucus than usual; appetite fair.

Treatment.-To have Kali Bichromicum, 20 drops, first dilution, three times a day.

This case steadily improved under the use of this remedy. At the end of three weeks from the commencement of the treatment he went to light work, and in another week went to his regular job.

\section{CAse III.}

On December 12th, 1858, I visited a horse belonging to Messrs. Taylor, Hampson, and Pegg, calico printers, Manchester. A fortnight previously the horse began to have a frequent cough, considerable difficulty in swallowing, and loss of appetite, for which Belladonna was given. Since then he has been getting worse, and being no longer fit to work, I was sent for.

Symptoms.-Pulse 50 and full; respiration 16 per minute; the nasal membrane, as far as it can be seen, is studded with numerous small, round, scarlet spots; there is a clot of muco-pus in the left nostril ; cough hard and frequent; eyes watery; profuse lachrymation from right eye; mouth dry; breath more unpleasant than offensive; tongue covered with a yellow fur; dung offensive; urine thick, and dirty-brown in color; hind legs very much swoilen especially around hocks; the swelling extends upwards for twelve inches, and ends abruptly: the fore legs are also swollen up to the knees; the hind legs are paddled uneasily, and there is stiffness and difficulty in walking.

Treatment.-To have Belladonna 1, 20 drops every three hours; sloppy bran mash; a few carrots ; very little hay.

13th.-Pulse 40, soft and full; respiration 10 ; some of the scarlet spots are fading; cough moist, and less frequent; tongue cleaner; appetite improved; swelling abont same, but not so painful. Continue medicine every four hours.

15th.- Some of the spots seem to have coalesced, for they are 
not so numerous, and are much larger, as large as a fourpennypiece, decreasing in size to a pin's point; the swelling in the legs is much less; the animal can walk with greater freedom; the appetite is good, and the excretions natural. Continue medicine.

18th. - Since last report the horse has been slowly improving; the spots are nearly all gone, except one about the size of a shilling; the swellings are reduced, and horse can walk about. Continue medicine.

December 21st.- I was sent for in great haste, as the horse was much worse. Pulse 50, and soft, full, and compressible ; the nasal membrane is quite black; the nose hard, swollen, and exquisitely painful ; respiration 16 , and snuffling; the swelling, which reaches as high as the stifle joint, where it terminates abruptly, is hard, hot, tense, and so tender that he will scarcely allow it to be touched; the fore legs are also swollen, especially at the knees; the borse can hardly move himself; he has a dull and dejected look, and eats little or nuthing; the bowels are costive, and the urine scanty and high colored.

To have Kali Bichrom. -1 grain in $24 \mathrm{oz}$. of water, $2 \mathrm{oz}$. for a dose, every two hours.

22d.-Much better; pulse 46 ; breathing 10 ; ecchymosis on the right nasal membrane has sloughed off, leaving a raw, ragged surface; that on the left is becoming detached; a piece about the size of a half-crown has sloughed off each nasal ala. The tumefaction of the muzzle is considerably less; the legs are much diminished in size, and not so painful; on the right side of the breast there is a hard, circumscribed swelling about the size of a goose's egg; on the left side of the chest there are two similar extravasations; appetite good; looks more lively.

Continue medicine every three hours. To have boiled oats, beans, and bran mixed, given frequently in small quantities, alternately with carrots.

23d.-Some shreds of slough are hanging loose in left nostril; others have been snorted out; the raw surface on the right nostril is filling up; the swellings of the legs are rapidly subsiding, and the horse walks freely about the box. A large slough is separating from the inside of both thighs, where the swelling was so great.

Continue medicine every four hours. 


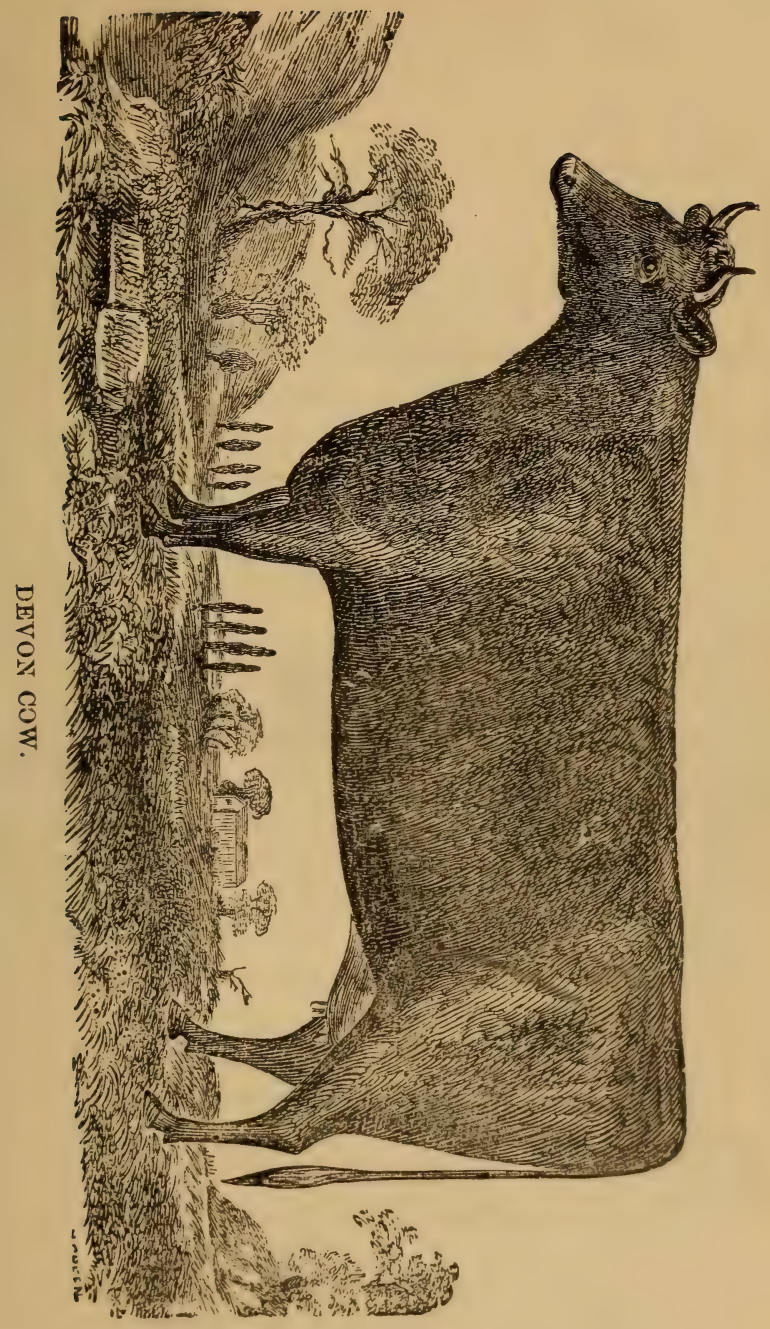



24th.-The sloughs on the left nostril have all been discharged, leaving a raw surface; the extravasations on side and breast are not so large.

Continue medicine three times a day.

28th.--The horse has been gradually improving, and is now quite well.

\section{7.-Milk Fever-Puerperal Fever-Dropping after Calving.}

This very dangerous, and in the ordinary practice, almost incurable disease, is much more common in cows and sheep than in mares. It is said not to be so apt to occur at the first time of calving, as on the subsequent occasions. High and stall-fed cows are most subject to this disorder, especially those supplied with rich food for the sake of making them give as much milk as possible. Milk fever sets in very soon after parturition, from the first to the fourth day; after the fourth day, if doing well, the cow may be considered safe. Sometimes its cause may be traced to violence; at others, to exposure to wet and cold; or the cause may be entirely indiscoverable. Mr Blaine says, "the treatment in the early stage calls for bleeding, and that liberally;" but in the early stage alone; and he advises a plenty of most powerful medicines. Gamgee, on the other hand, tells us, "above all things, avoid strong internal remedies and bleeding." Either method seems equally unsuccessful; for nearly all die that are taken, according to the testimony of allopathic veterinarian surgeons.

From Dr. Moore's original monograph we take the following account of milk fever, and its homøopathic treatment : 
Milk fever benins shortly after calving, and, in the majority of eases, within twenty four hours; the cow refuses ber food, or eats only a very little of it; she is depressed, hangs her head, and looks dull ; the horns are hot, and sometimes cold; the nose, instead of being damp with the healthy dew, is hot and dry; the urine is seanty; the bowels are contined, or, if moved, the dung is hard and lumpy; the pulse is quieker and fuller than in health; the breathing is quickened, and attended with beaving at the flanks. To these warning indications there suceed, with more or less rapidity, those unmistakable symptoms which are perhaps the first to attract the owner's attention. The milk is redueed in quantity, or entirely stopped; the eyes glisten, and look bright and staring; the white of the eye is covered with numerous red streaks, or it is of a leaden color; the eyeballs are thrust forward from their soekets, giving the cow a somewhat wild and anxious expression; the hind legs seem weak, and are separated a little from each other; she appears to stand uneasily upon them, first rests upon one for a short time and then changes to the other; this paddling and shifting about from one leg to its fellow continues until the diffieulty of standing increases, ahd the animal supports herself against the wall or stall; she does not chew the eud; all discharge from the bearing is stopped; the calf is neglected; the pulse is now slower than before, and the breathing more diffieult; the udder is hard and swelled, and little or no milk can be withdrawn from it. Gradually becoming worse, the weakness in the hind legs increases to so great an extent, that they ean no longer support ber: she staggers and sways about, falling, at length, heavily upon the ground; she 
tries to rise again, and may, or may not succeed; in either case, she soon loses all power of getting up, and remains upon the ground in a helpless state. In this stage of the complaint, the symptoms vary in different cases. In some cases the cow tosses her head about from one place to another, writhes her body, lashes her tail, struggles, stretches out her hind legs, moans, bellows, and appears, from the expression of her face and general behavior, to suffer great pain. At the same time, the breathing is difficult and labored; the skin covered with clammy sweat; and the paunch enormously swollen, owing to the stomach having entirely, or partially, lost the power of dissolving the food, which now undergoes the ordinary chemical changes, attended with the giving off of gas.

Unless the swelling which arises from the presence of this gas subside, the breathing becomes more and more difficult and labored, so that the animal can scarcely take her breath;* the pulse becomes oppressed, and can scarcely be counted at the jaw; the legs become very cold; more severe pain is felt; wind, having a bad smell, rises up from the stomach, and death ensues.

In other cases again these symptoms are altogether absent, or exist only in a slight degree; the more prominent being these: the cow lies stretched out full length upon her side, or her head is brought to the opposite side, with the nose towards the udder, and the chin resting upon the ground; or the head is twisted directly backwards, with the nose held out, and the horms turned upon the shoulder in a most awkward manner. The

\footnotetext{
*For directions for fecling the pulse of animals, ree the firmt chupter of this work.
} 
eyes look dim and glassy; upon placing a light near them, the cow takes no notice of it, and does not move or shut the eyelids, for the power of seeing is lost; the pupil is widened, in some cases almost round, and does not become narrower when light is held before the eye, as it does in the healthy state; the ears hang down; the mouth is partly open; and when the head is raised, the lower jaw drops down; the cow has not the power of keeping the head up when you raise it from the ground; the ability to swallow is nearly or quite gone; she has lost the sense of feeling; the breathing is still difficult, and attended with rattling in the throat; the pulse is weak, slow, sometimes stops beating for a moment or two and then goes on again, and, in some cases, can scarcely be felt at all; the horns, legs, and surface of the body generally are cold and chilly; the swelling of the belly increases; the udder is much swelled, hard, and sometimes red on the outside; in some cases, neither dung nor urine is discharged. All these symptoms become worse and worse, and if it resists all treatment, death ensues, generally within two days after the attack, and in some cases within a few hours.

The symptoms of this disease appear so suddenly, and run so quick a course, that the cow, about the calving time, should be narrowly watched, both night and day, in order that no time may be lost in opposing the complaint at its onset by the proper remedies. The delay of even a few hours may settle the question of the cow's recovery; the disease is then fully developed and death may ensue before the medicines have had a chance of acting. But even in the advanced stages the disease may be subdued. The author has had cases of recovery where the 
butcher was in attendance for several hours to slaughter the animal when at the point of death. Still, the cow is much more likely to rally if the disease be combated as soon as it begins. It behooves every farmer, therefore, to be prepared, and at once to give the medicines when the complaint declares itself. Several medicines are suitable for this disease, but the following six are, according to the author's experience, the most efficient, and should be kept at hand in case of emergency; they are Aconitum, Arsenicum, Belladonna, Bryonia, Ammonium causticum, and Nux Vomica.

Attention to the following directions will enable every cow owner to decide which of these medicines is most suitable, in any given case of this disease. He must first find out the symptoms or sufferings of his cow, that is to say, he is to inquire as to the pulse, breathing, milk, chewing of the cud, and general condition of the animal.

\section{Treatment.}

In the first place, then, supposing the following symptoms to be present: refusal of food; dullness and depression; hot horns ; dry, hot nose; scanty urine; confined bowels ; quick, full pulse; hurried, heaving breathing; wild, staring eyes; stoppage of milk; wild and anxious expression of countenance; paddling and shifting of the hind legs; eyeballs thrust out ; tossing about of the head; struggling and uneasiness of the whole body. For these indications of the disease the best and most successful remedies are Aconitum and Belladonna. The author believes that if they were given as soon as the lisease becomes manifest, almost every cow would recover. 
Dosfs.-Give Aconitum and Belladonna, alternately, in 20 drop doses, every one, two, or three hours, according to the severity of the symptoms.

In the second place, if the disease advances, and the symptoms are these: enormous swelling of the paunch; frequent, difficult, and labored breathing; gurgling and rattling in the throat; slow, weak, oppressed pulse ; cold, clammy skin; extreme coldness of the legs; lashing of the tail, tossing about of the head, and writhing of the body, showing that severe pain is felt; then give Ammonium causticum.

Dose.-Give 20 drops of Anmonium causticum, in a wine-glassful of water, every fifteen or twenty minutes, until the swelling goes down.

In the third place, if the symptoms just given remain, and the last medicine has had the effect of lessening the swelling; if, further, the cow is in the sleepy stage, and presenting the following condition : insensibility to pain ; loss of power of seeing, of swallowing, \&c. ; glassy state of eyes; open mouth; inability to hold up the head when it is raised; general coldness of the body, \&c.; then Arsenicum is to be given.

Dose.-Give 20 drops every fifteen, twenty, thirty, or sixty minutes, according to the violence of the symptoms, until improvement sets in, then lengthen the time between the doses to two, three, or four hours.

In the fourth place, if the cow has recovered from all the more urgent symptoms; if all traces of fever and of the sleepy stage have yielded to the foregoing remedies, but the animal still lies on the ground, and is unable to rise up, except upon her fore legs, then give $N u x$ Vomica, 
20 drops in a little water, every four hours, until the cow is well.

In the fifth place, if in the first stage the eyes are not bright, staring, or thrust from their sockets; if the udder is soft and flabby; in short, if the list of symptoms show that the head is not much affected as yet, Bryonia is to be given, 20 drops in a little water, every one, two, or three hours, according to the urgency of the symptoms.

Belladonna will be indicated by symptoms affecting the head. Eyes blinded, or dilated pupils, or bright, projecting, and glistening; by relaxation of the sphineter muscles of the rectum, the fundament and bearing are open and flabby; and by the udder red, hot, distended, and destitute of milk.

In the sleepy stage, when the power of swallowing is gone, and when the cow would choke were large quantities of fluid introduced into the mouth, the medicine, in 20 drop doses, ought to be given in half a tablespoonful of water, instead of a wine-glassful. The advantage of our remedies is here apparent. During this stage, when giving the medicine, care must also be taken not to hold the head too high, otherwise the animal may be choked.

GENERAL DIRECTIONS.

1. The cow should, if possible, be placed in a large box or stall by herself, where there is plenty of fresh air, so that she may breathe freely, and have room to make attempts to rise, which she will frequently do when recovering.

2. She should be laid upon clean, dry straw, and no dung should remain long.

3. She should be comfortably clothed, according to the state of the weather. 
4. The milk should be frequently drawn off, and the udder hand-rubbed.

5. If she makes no water, the catheter should be passed to draw it off, at least night and morning.

6. Care must be taken that the fore part of the cow is rather higher than the hinder, or that she lies upon an exact level, with her legs under her, as in the natural position. She must be placed rather upon one side, and well supported with bundles of straw. If she do not turn herself, she must be shifted from one side to the other every two hours. She must on no account be allowed to lie stretched out full length on one side, else she will surely become worse, and die from suffocation. Her head should be supported with bundles of straw.

We subjoin a couple of Dr. Moore's cases, to show his method of treating this disease, and its great success.

\section{CASE I.}

A cross-bred, six years' old cow has been in the pussession of the Rev. Mr. Figgins, Booth Cottage, Blackley, near Manchester, for a considerable time, and has always been well. About twenty-four hours after calving, she dropped, and was quite unable to rise up. Mr. Figgins, who has always a supply of medicines by him, lust no time in giving several doses of Aconitum and of Belladonna before my arrival. This promptitude of treatment kept the disease in check, for, on examining the cow, I found that all the worst symptoms had yielded.

The symptoms are:-Pulse 76 and strong; respiration hurried; she is lying easily upon the ground, but is quite unable to rise; her eyes are glistening, wild-looking, and projecting; there is no milk, appetite, chewing of the cud, etc.

Treatment.-To have Nux Vomica every three hours.

Much better; has chewed the cud; would eat more than is given, making bold efforts to rise.

Continue medicine. 
She got up last night; gives milk freely; all the functions are again healthy.

\section{CAse II.}

A seven year old, very highly fed cow, belonging to Mr. Joshua Marler, Newton Moor, near Manchester, about twenty-four hours after an easy delivery, her fifth, began to show all the warning symptoms of this disease, and I was sent for immediately.

Symptoms.--The pulse is slow, feeble, and quickened; the udder is hard, distended, hot, and yields no milk; no dung or water comes away ; the fundament and bearing are open and flabby ; the body is occasionally rolled and writhed about uneasily, and the tail lashed: she sometimes tosses herself from side to side, but is, of course, quite unable to rise; the belly is swollen; she cannot hold her head up; the eyelids are half closed; the pupils dilated; tears roll down the face - in short, without enumerating others, she has all the symptoms of the sleepy stage.

Treatment.-To have Arsenicum and Belladonna every hour, turn about, until symptoms of improvement show themselves, then every two hours.

May 21st.- All the above symptoms are much relieved; the swelling is gone down; she can hold up her head; has dunged and made water; drank some gruel, etc.

Continue medicine.

May 22d.-She lies like a cow resting, to all appearances well, only unable to rise.

To have Nux Vomica every three hours.

May 23d.--Got up this morning; all right.

\section{8.-The Liver of the Ox.}

This organ is situated on the right side of the abdomen, between the manyplus and the diaphragm. It is principally supported by a duplicature of the peritoneum extending from the spine, and is confined in its situation by other ligaments, or similar peritoneal duplicature, connecting its separate lobes or divisions with the dia- 
phragm. It is divided into two lobes of unequal size. The right lobe is larger than that in the liver of the horse; the smaller one is comparatively diminutive; and, altogether, the liver of the ox is less than that of the horse.

The blood from the other contents of the abdomen, instead of flowing directly to the heart, passes through the liver. It enters by two large vessels, and is spread through every part of the liver by means of the almost innumerable branches into which these vessels divide. As it passes through the liver, a fluid is secreted from it, called the bile, probably a kind of excrement, the continuance of which in the blood would be injurious, but which, at the same time, answers a peculiar purpose in the process of digestion, that will be presently described.

The bile thus secreted flows into the intestines, and enters the duodenum through an orifice. In the horse, it flows into the intestines as fast as it is secreted or separated from the blood; but in cattle, a portion of it, probably a comparatively small portion, is received into a reservoir, the gall-bladder, where it is retained until needed for the purpose of digestion. While the ox is grazing or asleep, there is no necessity for the whole of the bile to run on into the intestines, but a part of it accumulates in the gall-bladder. While it is retained there, it undergoes some change; part of the water which it contains is absorbed, and the residue becomes thickened, and more effective in its operation; and when the animal begins to ruminate, and portions of food pass through the fourth and true stomach into the duodenum, not only is the flow of bile into the gall-bladder stopped, but, either by some mechanical pressure on that vessel, which no one has yet explained, or, more probably, by 
the sympathy which exists among all the organs of digestion, and the influence of the great organic nerve causing the (probably) muscular coat of the vessel to contract, the bile flows out of its reservoir, and proceeds to its ultimate destination, along with the portion which continues to run directly from the liver into the intestine, through the medium of the hepatic duct. This pear-shaped reservoir, the gall-bladder, is placed in a depression in the posterior face of the liver, and adheres to it by means of a delicate cellular texture. The construction of this vessel deserves attention. It has the same external peritoneal coat with the viscera generally ; beneath is a thicker coat, evidently composed of cellular substance, in which no muscular fibres have yet been demonstratively traced, but in which they may well be conceived to exist, and in which, doubtless, they do exist, in order to enable the gall-bladder to contract and expel its contents. The inner coat is a very singular one. It has not precisely the honeycomb cells of the reticulum in miniature, but it is divided into numerous cells of very irregular and different shapes, in the base of which, as in the cells of the reticulum, are minute follicular glands, that secrete a mucous fluid to defend the internal surface of the gall-bladder from the acrimony of the bile which it contains.

\section{9.-Lymphatitis, or Weed.}

\section{IN HORSES.}

Weed is one of the common names for a rather frequent disease in horses, consisting of inflammation of the lymphatic vessels and glands of the legs, especially 
a hind one. It is most frequently observed in carthorses, and is caused indirectly by errors of feeding, and directly by injuries, exposure of the legs to damp and cold, etc. I have noticed that many horses are attacked on a Monday morning, after the previous day's rest.

Usually, the attack comes on suddenly with a shivering fit, followed by feverishness - quick pulse and breathing, hot, dry mouth, etc. The affected leg is raised from the ground, and the horse frequently looks round to it, as if to tell where his pain is. The leg, on examination, is found to be hot, swollen, and tender to the touch. On the inner side of the thigh and leg we discover a hard, painful, cord-like enlargement along the course of the lymphatic vessels, with here and there several enlarged glands or valves in these vessels. The swelling of the vessels and leg begins in the groin, at the bend of the thigh, and gradually creeps downwards along the inner surface of the leg, as far as the hockjoint, or even to the fetlock. The tumefaction of the leg pits on pressure, and in severe cases an amber-colored fluid oozes out from the skin, and stands in drops on the hair. One attack predisposes to subsequent ones, and the leg is left permanently enlarged.

\section{Treatment.}

I have treated many cases of both these diseases, and at the time I write have just seen several improve rapidly. Several remedies have been employed, but of late I rely exclusively on Kali Bichromicum, 20 drops every three hours. I also cause Kali Bichrom. Lotion to be rubbed in, night and morning. In some cases, I first give a few 20 drop doses of Aconite. 


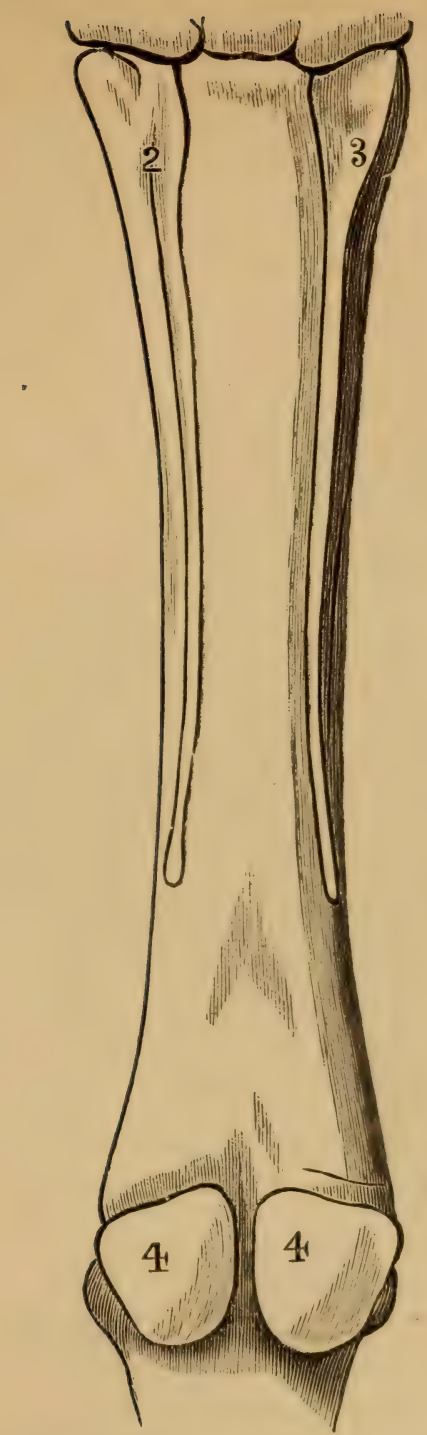

1, Great metacarpal bone.

2, Outer small metacarpal or splint bone.

3 , Inner small metuearpal or splint bone.

4, 4, Sesamoid bonss. 



\section{0.-Cellulitis.}

This disease is often confounded with weed, from which it may be distinguished by the absence of the enlarged vessels, valves, and glands in the inside of the thigh; by the swelling first appearing in the fetlock and extending upwards to the hock, sometimes to the thigh ; by its attacking equally any leg; and by the skin breaking at the most prominent part of the swelling, and giving vent to a large quantity of foul matter. A slough is cast out from the opening, and a deep, unhealthy ulcer remains. At the same time, the animal is feverish and his breathing is quickened; the freces hard and coated with mucus. The leg is excessively painful to the touch, and frequently so hard that the swelling does not pit on pressure with the finger. One attack, like weed, predisposes to subsequent ones, and the leg is left permanently thickened.

\section{Treatment.}

I give a few doses of Aconite, 20 drops every three hours. Then Kali Bichromicum and Carbolic Acid as directed for weed; also rubbing in the lotion of the same drug. The result is rapid recovery.

\section{1.-Foot Rot in Sheep.*}

On swampy, spongy soils sheep are particularly liable to this disease, which, though exclusively almost local, is one of the most severe and even revolting character

* Pigs are also more or less liable to this diacase, with very analogons characteristies. 
If sheep be transplanted from firm, dry, upland soils to deep, low, saturated marsh lands, they will be still more likely to suffer. Foot rot is communicated by contagion, that is, by poisoning with the discharge.

Symptoms. - The reversion of the outer edge of the crust upon the sole generally, or on one side only ; symptoms affecting the fore feet in particular, with more or less rapid progress, sometimes returning from time to time; symptoms affecting one or more of the feet simultaneously, and sometimes finally attended with unaccountable lameness, and tenderness of the foot, and softening of the horn; detachment of parts of the horn; parting of the crust from the sole; fissures, and dislodgment of the crust and horn, generally exposing the quick, and for some time the continual reformation of a horny coating; enlargement of the coronet; ulceration of various parts of the foot, discharging thin but offensive matter; proud-flesh developed by the ulceration; the inflammatory and suppurative process varies in the period of development; sometimes appearing immediately, and sometimes slowly provoked by the irritating causes connected with the growth of the crust; separation of the coronet and hoof; heat, swelling, inflammation, and ulceration of the interstices of the foot; oily, fatty discharge, concreting with dirt, over the whole of the foot; gradual detachment of the hoof by the internal ulcerative process ; the pasterns are affected, and become raw ; there is general swelling of all adjacent parts; more or less constitutional disturbance ensues.

The great importance of this disease requires a fuller account of its milder and severer forms, which we quote from Gunther. 
The mild foot rot of sheep, usually associated with Stomacace or ulceration of the mouth, gradually extends to entire flocks. It commonly begins with fever, more or less violent, which sometimes continues during the course of the disease, and is recognized by symptoms, of which the principal are the following: The animal of a sudden becomes sad, and limps or one or more feet; there are heat, redness, and swelling of the feet, chiefly at the interdigital space, and on the coronet. At a later period the inflamed points are ulcerated, and on the cushion appear vesicles, which "at first secrete a fluid clear as water, and pus at a subsequent period. The disease progresses with great rapidity. It generally disappears of its own accord in a few days. In order to accelerate the cure, however, and render it more certain, the foot is to be washed frequently with warm water, the superfluous horn is to be removed when it projects or is in any way altered, and Arnica is to be employed internally and externally.

Malignant foot rot is known by the following indications: The animal begins to limp, sometimes at first in one of the fore legs or hind legs; sometimes in the two fore legs and two hind legs, until the whole four are affected. The diseased foot is hot and a little swollen; the clefts are separated a little more from each other than in the healthy state. The skin of the interdigital space is red, and exudes a fluid of a bad odor; this fluid gradually assumes the appearance of ichor, which not only inflames and excoriates the surrounding integuments, but becomes effused also behind the horny wall, which is separated in part or entirely from the living parts; sometimes even the integuments, tendons, and the very bones 
are involved. The animal, then incapable of walking, moves along on its knees, or remains lying down, and wastes away gradually, though retaining generally a good appetite. This form is very contagious, so that when the sheep just attacked is not removed away from the flock, all the others soon become affected. To propagate the disease, it is quite sufficient that a flock should pass over a place which has a little before been walked over by a diseased sheep.

\section{Treatment.}

In the milder form, and in the earlier stage, as soon as limping is observed, the animal should be examined, and if there be any signs of the separation, especially of the crust from the sole, or of the coronet from the horn, the first prevention will consist in providing the animal so affected with a separate inclosure, lest others be infected, and in carefully removing all dirt which may have been introduced into the fissures, after which the following local application will, with proper precautions, generally prove sufficient to arrest any further progress of the disease.

For an application to the foot, use four parts oil of tar and one part oil of vitriol, applied with a small swab or brush, after cleaning off the foot and cutting away the dead hoof. If this should fall, use Butter of Antimony.

Tincture of Calendula, constituting a lotion, by the admixture of four parts of water to one of the tincture, should be applied to the parts every three hours for the first day, and three times in the course of the second. Twelve hours should then be allowed to elapse 
before constitutional treatment is adopted, when resort to Carbolic Acid lotion.

In the severer form, the diseased part must be scraped to the quick with a sharp knife. It has been advised by Gunther to bathe the wound and ulcer with salt water and dilute Nitric Acid; and by others to employ, when the ulceration is more extended and the disease very malignant, the stronger dilutions of Arsenicum causticum and Ammonia. But we are quite sure that the dilute Carbolic acid, one part to forty of water, will do much more good, and advise to use this in preference to all other local applications in this disorder. Relapses, which may result from want of sufficient thoroughness in the paring, cleansing, and bathing with the medicated lotion, must be treated as at first. And for a considerable period after apparent recovery the affected animals should still be retained in inclosures by themselves.

The internal medication should consist in the administration of Arsenicum, Nitric acid, or Ammonium causticum, ${ }^{*}$ according to the other symptoms and condition of the patient.

Dose.-The selected remedy should be given in six-drop doses once in three hours; when improvement appears, once in four hours.

The diet should be nourishing but not stimulating, such as mashes and boiled vegetables.

The local applications must be repeated at intervals of three or four hours.

* If pref rred, one dram of the strong solution of Carbolic acid may be placed in a cuart bottle, and one pint of water gradually added, shaking the whole vio. lently with each successive addition, and of this mixture one tablespoonful may be given every two hours in cases of malignant foot rot, in conjunction with the external use of this antiseptic, as already laid down. 


\section{2.-Navicular Disease, Groggy Lameness.}

Many obscure cases of lameness in horses are caused by disease of the navicular bone, and strain of the flexor tendon that passes over it to be inserted into the bottom of the coffin bone. This navicular bone (a sort of large sesamoid bone) is placed behind and beneath the lower pastern bone, and behind and above the head of the coffin bone.

The symptoms of navicular disease in confirmed cases are very peculiar. In the stable it is indicated by the horse standing either with his legs straight out and bearing his weight on his toe (the heel being raised off the ground); or by the fetlock being flexed, whilst the toe is drawn back and stuck into the ground. In action it is manifested by the animal treading chiefly on his toes, and consequently digging them in the ground. When the horse has been shod a week, the extra wear thus occasioned will have made itself perceptible on the toe of the shoe. The object of the horse in all these peculiar positions and motions is to avoid putting weight on the back part of the foot, which is the seat of the disease.

In cases of long standing, the inside quarter of the foot will become straighter, and there will be small, circular ridges on that part of the hoof. The horn of the sole will also be found to be increased in quantity, and the foot and the sole will be more concave than natural. From saving of the back part of the foot and consequent absence of pressure on the frog, thrushes will probably make their appearance. Navicular disease may and very often does appear in good, open feet, but gradually the above changes will take place. 'Tenderness will be 
evinced on the application of concussion to the heels, or at the point of the frog. A further test may be applied by bending up the foot and applying pressure by the thumb to the hollow of the heel. 'The seat of the disease will be nearly under the thumb.

The lameness is most apparent, when the horse first comes out of the stable. It decreases. with exercise. Nevertheless, on the day after severe work, the horse will be more lame than usual. In doubtful cases, therefore, the animal should be subjected to strong work, and examined again on the following day.

\section{Treatment.}

The cure of navicular disease, according to Youatt, is difficult and uncertain. But the homoeopathic remedies show here to great advantage, both for the disease of the bone (of which the cartilage may be ulcerated, and the bone itself carious), and of the ligament which plays over it. Only where such ulceration has already given rise to adhesions and other permanent disorganizations, the medicines can have little effect. Perfect rest must be enjoined. Inflammation, shown by the heat of the foot, must be removed by repeated doses of Aconite; give ten drops every four hours.

Rhus tox. should be given in similar doses, whenever there is reason to suspect a strain or other injury of the flexor tendon. Many cases of lameness in horses, whose proper origin and seat and cause even remain unknown, are permanently cured by this invaluable remedy.

Acidum Phosphoricum may be required when there is reason to suspect inflammation of the cartilages. Silicea is indispensable in most cases of advanced navicular dis. 
ease; this remedy, with Phosphoric Acid, may remove the difficulty, even after ulceration of the cartilage and caries of the bone have occurred.

Antimonium crudum and Sulphur have also been recommended in this disorder.

D osE.-Of the selected remedy give twenty drops in a little water, three times a day.

I have found an excellent remedy for this disease in the patent shoe, as given on the plate. Soak the foot three days in warm water, then put on this patent shoe, spreading it as far as needed to relieve the foot, but not more than one-fourth of an inch at a time. Use for an outside application oil of tar and neatsfoot oil, in equal proportions. Fresh butter, or sweet oil and oil of tar may be substituted for the above. Apply twice a day, until the foot assumes its natural shape.

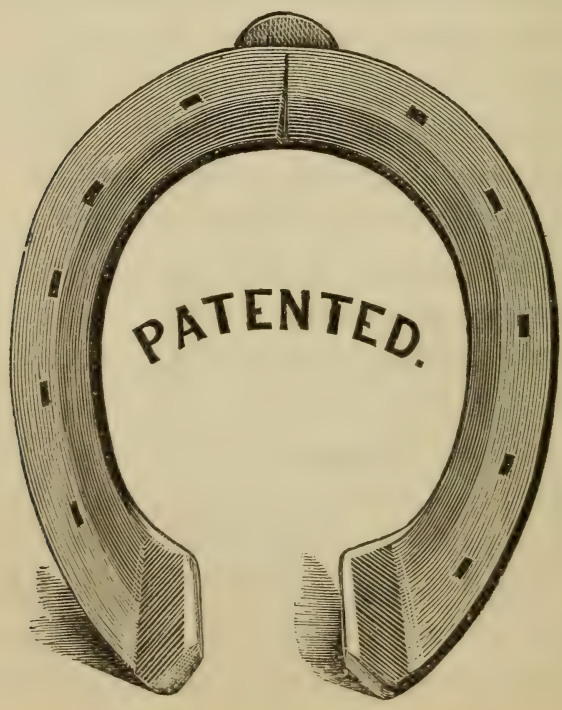




\section{3.-Thrush.}

This affection of the feet of horses is recognized by the discharge of very fetid matter from the frog (from the interstice of the frog). Inflammation of the sensible or inner portion of the frog is the direct cause which provokes the secretion of such matter, and this soon becomes abundant and offensive. The cleft of the frog appears deepened, and assumes the appearance of a crack. Contraction may be considered both as the cause and effect of thrush - in its former relation - by exposing the frog to additional and severe pressure, whereby inflammation is provoked. With respect to thrush, as regards the fore feet, it may generally be attributed to this cause. The hind feet are, however, still more frequently affected with thrush, which is attributed to the exposure of those parts to the injurious effect of the foul moisture, which may, from want of proper attention, be allowed to accumulate at the lower part of the stall. The tenderness of the frog in this disease may result in serious lameness and aggravation of the disease, by the feet being bruised on the stones.

Thrush as an affection of the feet, peculiar to horses, must not be confounded with aphthæ, or thrush of the mouth, which latter affects various domestic animals.

\section{Treatment.}

Calendula Lotion, Arsenicum, 3d dilution, and saturated tincture of Sulphur have been advised for local applica. tion. And they are are all capable of doing good ser- 
vice: Especially the Arsenicum, when the discharge becomes putrid, and the parts assume a gangrenous appearance.

But we regard the Carbolic Acid Lotion, as already advised in other putrid and malignant affections, and in the foot rot of sheep, as being far superior in its influence in this affection to all others. Bathing the inflamed and ulcerated surface with this lotion, prepared as already directed, will best co-operate with the internal exhibition of the appropriate constitutional remedy.

Strict attention to cleanliness, and placing the animal on a dry floor, are essential to his recovery.

Kreosote.-20 drops, four times a day, will be found very useful in healing the affected feet and counteracting the morbid influence in the system, which produces thrush. Very similar to Carbolic Acid, Kreosote is an excellent remedy to give internally in conjunction with the external application of Carbolic Acid Lotion.

Phosphor. Acidum is also to be studied in such cases; especially, where the inflammation threatens to involve the deeper tissues of the foot.

Dose.-6 drops, four times a day.

Give light and unstimulating, but nourishing food; rest and perfect cleanliness, with the external and internal medication, will almost invariably perfectly cure this disease in a short time. When it is most obstinate, Sulphur, or other constitutional treatment, must be persevered in for weeks, as the difficulty with the feet may be only a manifestation of poor condition of the animal in other respects. 


\section{4.-Rinderpest-Cattle Plague of Great Britain.}

The Rinderpest, or, as this German name is literally translated, the Cattle Plague, belongs to the class of zymotic diseases, those in which,--according to the former pathology, the blood was believed to undergo a ferment. It is equally contagious, malignant, and fatal. It is a blood disease; but the blood experiences a change much worse than fermentation; for it becomes infected with the virus of the disorder, so that every single particle of the blood from an affected animal becomes capable of infecting all the blood of any other animal of the ox kind, with which it may be brought into contact. The rinderpest took its rise, according to authentic history, more than one thousand years ago, in the vast, elevated plains (steppes) of Asiatic Russia. And how many hundreds or even thousands of years further back it may have prevailed-the same infectious, malignant disorder, although somewhat various in the form of its manifestation-is still a matter of dispute. During the last hundred and twenty-five years many tens of millions of cattle have perished from this disorder in Europe alone. The following is a brief account of this form of murrain, as it appeared in Great Britain in June, 1865 ; brought by some Esthonian cattle imported feom Copenhagen, and which, in the short space of nine months, destroyed three hundred thousand cattle.

Symptoms.-The period of incubation varies with the mode of introduction of the poison; where the disease has been inoculated, it makes its appearance in four or five days; when it is caught in the usual manner, in 
from eight to ten days. Within thirty-six or forty-eight hours after inoculation, the blood is so thoroughly contaminated that a single drop is sufficient to develop the disease in all its malignity, when employed as an inoculative medium for another animal.

Primary and Successive Appearances, and External Symptoms of First Stage.-When first taken, the animal loses its appetite; ceases to chew its cud; gradually becomes constipated; the dung is of a dark color and sometimes covered with slime; cows diminish their flow of milk. The animal stands in the same posture; looks depressed; with drooping head and reclining ears. There is also loss of the natural heat. The beast is remarkably heavy and dull; hangs its head, lowers its ears, stays behind the herd, and when in the stable keeps away from the crib. The head sometimes shakes to and fro; if lifted up, it goes down again like a dead weight ; there appears also an uneasy, excited condition; the animal acts as if in pain, stamps its feet, frequently lows, butts with its horns, and runs away from the herd. Trembling motions occur; the hairs bristle up; the insertions of the horns are sometimes cold, sometimes warm; the palate is dry; the eyes shining. Respiration is slightly quickened; there may be cough, with great difficulty of breathing, the animal making more noise on expiration than in pleuro-pneumonia. The vulva assume a reddish tinge, of which the color deepens as the disease advances. The mouth shows a faint red or purple line on the under gums along the roots.

In the second or congestive stage the pulse rises (from the healthy standard of forty-five to sixty, in the field and stable respectively) to eighty, ninety, or even one hun- 
dred and ten beats in a minute. Respiration becomes from forty to ninety-six, instead of eighteen or twenty per minute, as in health. Temperature lowered and vitality depressed, characterize this disease throughout its course. Exudations from the eyes, nose, mouth, and vulva, form with rapidity, consisting of a glairy, ropy mucus. An abundant yellow or bloody, stringy discharge comes from the nostrils, which gradually becomes white and fetid, and a tough, viscid slime flows from the corners of the mouth. The anus is frequently highly congested; the urine becomes loaded with blood, and is passed with considerable pain and difficulty.

With convalescence the animal improves in appearance; begins to take food and chew the cud. An itching, scabby eruption sometimes appears on the skin of the nape or sides of the neck, or on the back. But when the disorder takes an unfavorable turn, and the period of congestion is not relieved by favorable indications, then follow the symptoms which result in death. Diarrhoea, often dysenteric in its character, or thin, watery, and offensive in the highest degree, sets in; exhaustion, accompanied by intense restlessness, follows, and death takes place in consequence of the failure of the vital forces, which have been overwhelmed in the putrefactive dissolution of the fluids and solids of the body. Sometimes where the symptoms seem to have been improving, the animal suddenly becomes more dull, the head drops, the eyes look heavier, the conjunctivæ are almost livid, the teeth are ground, the animal butts at everything within its reach, oftentimes becoming furious, and suddenly dies.

With the following description of the course of the 
discase in a cow that had been inoculatedby inser ting in both sides of the neck between the shoulders woolen threads saturated with the secretions from the eyes of a yearling calf, we close our account of this malignant disorder: "On the sixth day, short, hacking cough. On the seventh day, loss of vivacity, drooping head and hanging ears; rumination ceased; shaking of the head; gnashing of the teeth; hair bristling, and skin lying in folds. On the right side auscultation showed blowing murmurs; and percussion, dullness. On the same evening, eyes and nose began to run; milk diminished, and had a strong, salty taste; respiration and pulsation equal to sixty per minute. On the eighth day these rose to eighty; secretions increased; ears and horns alternately hot and cold; mouth hot; chilliness over the whole body; neither ate nor drank. On the ninth day respiration one hundred, with sighing; left side of bowels tympanitic, but normal at six P. M., when the pulse was eighty-eight; diarrhœa mixed with blood; aphthous appearance of the vulva. On the tenth day, pulse one hundred and eight; respiration fifty,-and sighing; bloody diarrhœa; colliquative flux from nose and eyes ; body cold, and death at six P. M."*

The moroid anatomy of this and many other similar cases is well described in the report already cited, and made clear to the eye by numerous colored lithographs.

Says Professor Gamgee: "Little can be done in the way of treatment beyond, etc., etc.; but all treatment

* "If the disorder continued beyond the seventh or ninth day, if the breath continued hot and the body cold, and the discharge from the eyes an i nose increased, the animal appearing in pain, death was usually near."-From Dr. Lagard's Account of the Murrain in England in 1757. 
appears futile." Such is the honest confession of the old school, or allopathic veterinary practice. It remains to show with what success homœopathy has struggled with this deadly plague. But before proceeding with this, it is proper to refer to a method from which, at one time, great results were expected-inoculation. The plan is the same as inoculation with small-pox in men, which was deemed a great discovery, till superseded by Dr. Jenner's safer and equally certain method of vaccination, or inoculation with cow-pox matter. Inoculation for the cattle plague failed for the same reason that the inoculation with small-pox matter failed, that many cases proved fatal. And this method of attempting to arrest the progress of the rinderpest proved less successful just in proportion to the greater malignity and fatality of this disorder.

\section{Treatment.}

The remedies which were found most effectual in treating the cattle plague of Great Britain are reported by James Moore, M. R. C. V. S., and A. C. Pope, M. D., the former a well-known veterinary surgeon, and the latter an eminent physician, who took great interest in practically studying the treatment for the sake of testing the power of homoopathic remedies to cope with this malignant disease. And from the reports of these gentlemen we glean some hints as to the particular indicacations of the remedies employed.*

Arsenicum.-This remedy was largely given as a prophylactic; but although it was at first believed to have exerted a favorable influence in some cases, and

* Monthly Homœopathic Review, March and February, Isiti. 
possibly to have warded off the disease in a few instances, its positive value to prevent the cattle plague generally is more than doubtful. In Mr. Moore's hands Arsenicum proved successful in one case, in which the the animal had running from the eyes and mouth, and loss of appetite and milk. In another case, where the cow was enormously emphysematous all over, except the ears and tail, so much so that the pulse could not be felt; when the respiration was 80 per minute; great difficulty of breathing; the paunch much distended; the nose poked out, and the fæces slimy, Mr. Moore saw little hope of recovery ; but prescribed a teaspoonful of Liquor Arsenicalis and the same dose of Liquor Ammonioe, every two hours in alternation, with perfect recovery. Dr. Pope found Arsenicum chiefly useful in meeting the prostration about the fifth or sixth day.

Dose. -20 drops thoroughly mixed in a little water; two tablespoonfuls to be given every three hours.

Belladonna.-According to Dr. Pope, Belladonna more than any other remedy corresponds to the prominent features of the English cattle plague. The difficult breathing, the congested mouth and throat, the engorged conjunctiva, the general congestion which pervades the mucous surfaces, with desquamation following, all point to this as the remedy par excellence. It has been, he says, "more valuable than any other we have used." The first, second, and third dilutions were tried in the early cases, but they were by no means so satisfactory in their action as the pure tincture, which was given in from four to ten drop doses every two, three, or four hours. 
Mr. Moore reports a case in which he gave Belladonna and Bryonia with success. The following were the symptoms: Pulse 72 ; respiration 50 ; crepitation in left lung; the membranes of the eye and nose reddened; the nasal wings swollen; the muzzle spotted with purplecolored spots; running from nose and eyes; the vagina reddened; the appetite and lacteal secretion diminished; cudding suspended. Another case, a cow suffering from well-marked symptoms of the plague, such as discharge from the eyes and nostrils; redness of the vagina in stripes; pulse 76 ; respiration 64 ; crepitation in left lung; little appetite; rumination suspended; diminished secretion of milk - recovered under Belladonna and Phosphorus. A cure was effected with these same remedies of another cow presenting the following symptoms: Pulse 72 ; respiration 60 ; crepitation in right lung; violent purging of thin, offensive fluid; eyes sunken, and discharging muco-pus; nasal membrane very red; discharge from nostrils; short cough; head protruded; milk gone; cudding suspended; vagina red and mattery.

Phosphorus was equally valuable with Belladonna, and given in alternation with it, in cases where the lungs became decidedly involved, and yet there was not the profound prostration which required Arsenicum. Dr. Moore* reports a couple of cases cured with Phosphorus and Belladonna. These cases have been cited under the head of Belladonna. The cattle plague in Holland was successfully encountered by Phosphorus in the sixth dilution. It should be given in twenty drop doses, well mixed in a little water.

* Monthly Homœopathic Review, March, 1866, p. 154.

16 
Rhus tox.-The chief indication for this remedy, according to Dr. Pope, has been found in the muscular twitchings which characterize this disease in some of its stages.

Dose.- Same as for Arsenicum.

Mercurius has been found useful when the mouth has been long congested, and the patches of desquamation are general.

D ose.-As advised for Arsenicum.

Ammonium Causticum, first decimal, is of service when there is much abdominal distention, with heavy breathing and painful moaning.

Dose.- 12 to 20 drops in a little water, every hour, till relieved.

Terebinth (Turpentine), first decimal, has been of signal service in checking hæmaturia, a symptom which did not yield to Cantharis.

Dose.-- As above indicated for Ammon. Caus.

Phosphorus Acid, first decimal, Mercurius Solubilis, and Arsenic have appeared to control the diarrhœa more than any other remedies, according to Dr. Pope; "but they have not proved altogether satisfactory. In a future case I should be disposed to try Muriatic Acid or China. It has been a more difficult symptom to meet than any other." This diarrhœea must always be an incurable symptom in the last stages of the disorder; then it simply results from the final decomposition of the tissues and dissolution of vital solids and fluids alike.

In addition to the above mentioned remedies, others, called antiseptics, such as Phenic Acid (the original name for Carbolic Acid), Condy's Fluid (a solution of Perman- 


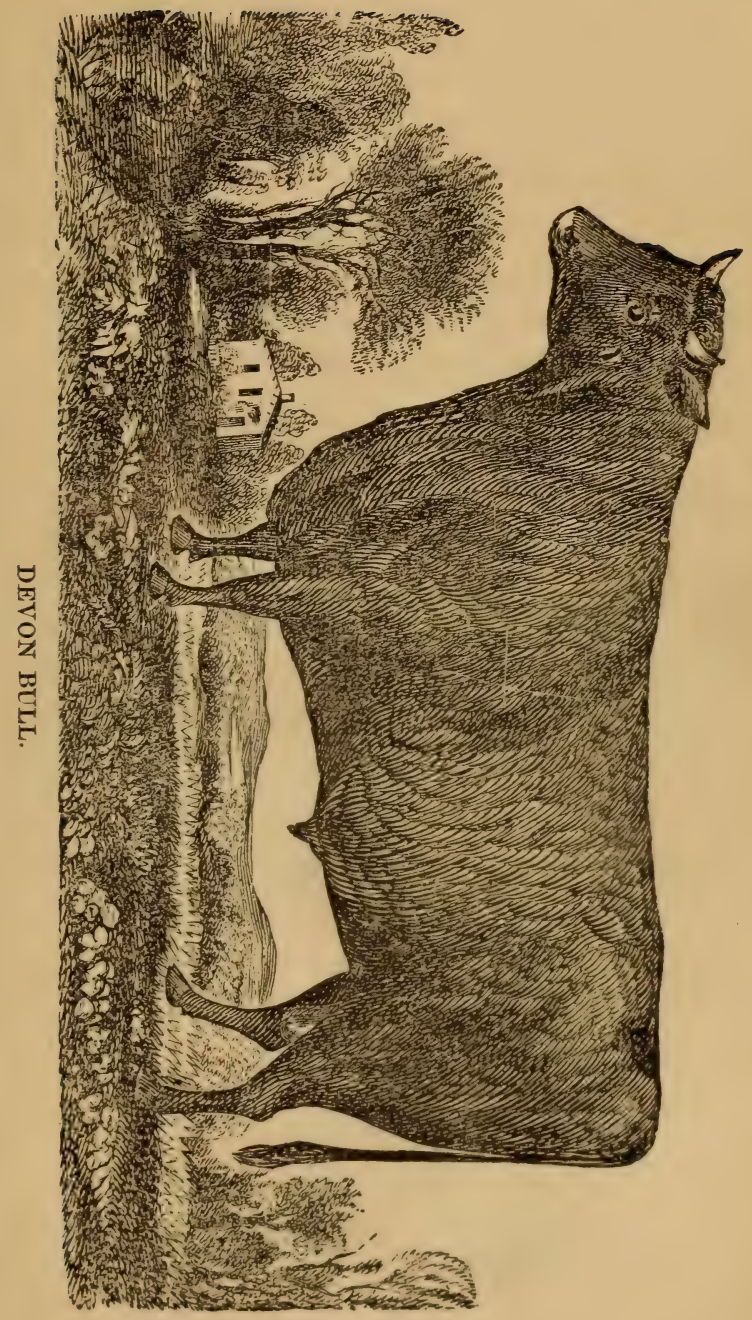



ganate of Potassa), and Arsenious Acid, in the form of Liquor Arsenicalis (Fowler's solution), have been employed with great success. "Many of the veterinary surgeons in Holland believed that the disease was of parasitic origin, and on that ground they tried the Phenic (Carbolic) Acid, and with considerable success."* Later experience, especially in the United States, demonstrates the power of these remedies to destroy the virus of the cattle plague, externally and internally; but the Phenic (Carbolic) Acid is by far the most efficacious of all. And the use of this internally in this class of infectious disorders renders it for them an abortive or preventive treatment.

For further views of the antiseptic treatment of this malignant and infectious disorder, see the "Treatment of the American or Texas Cattle Plague," in the following section.

\section{5.--"Texas Cattle Disease" - "Spanish Fever" - The American Rinderpest.}

In June, 1868, a cattle disease appeared at Cairo, Illinois, which was quickly recognized as that called "Spanish Fever" in the Gulf States. This scourge, previous to the late war, had repeatedly crossed the Texan border, and swept off immense numbers of cattle in Kansas and Missouri. From Cairo it now spread through the interior of Illinois and Indiana, and made itself known along all the great lines of communication between the West and New York city. It was brought

*Monthly IIomœopathic Review, Dec, 1865, p. 746. 
by cattle driven from Texas, and hence known as the "Texas Cattle Disease." But the infection conveyed by these animals to the "native" cattle of the States through which they passed was vastly more fatal to the latter than to the former. Indeed, it was affirmed at first, that the wild cattle from Texas, though imparting the disease to others, themselves 'constantly escaped; but this was soon disproved. From the very able, elaborate, and complete Report of the New York State Agricultural Society, 1867, Part II, we have derived the material of the following statement of the symptoms and course of this disease, often employing in whole paragraphs, and descriptions of individual cases, the very words of the writer. And to this Report, illustrated as it is with numerous colored plates, the reader is referred for an exhaustive account of the natural history and pathology of the Texas cattle plague.

Origin.-The causes which originally developed the disease in question among the savage cattle owned by thousands in the wilds of Texas, it is not within our province to discuss. Suffice it to say, in numberless instances, steers selected at random from droves of Texan cattle, which were to all external appearance in perfect health, would present, on being slaughtered, "the unmistakable scars which this disease invariably leaves upon the coats of the stomach." And the hardships and privations of the long journeys which these poor animals were compelled to make - unable to sleep and deprived of all food, and even water for many days in succession - sufficed to develop the seeds of this disorder, already present in their systems, into a state of virulence which destroyed whole herds, and in some instances completely 
exterminated the domestic cattle in the regions through which these herds were transported.

Nor was the manner in which the infection was communicated to what in contradistinction from the Texan droves were called "native" cattle, the least remarkable circumstance in the history of this American rinderpest. Native animals mingling freely with these Texan strangers would of course receive from them personally whatever of infection they had to impart. But in the great majority of instances in which this plague was communicated, there had been no such personal intercourse. But wherever the foreign cattle had traversed the roads, or been pastured, or even herded over night, their droppings retained so much and so active a form of the virus, that all native animals that occupied the same ground, even weeks or months after, rapidly sickened and the greater number of them died.

The poison was apparently communieated to them through being absorbed by openings in the cleft. For native cattle have been known to take this disease from being only driven over the same roads which had been traversed recently by those from Texas. In other cases there is no doubt that the disease is propagated through the excrements (droppings) coming in contact with the food, grass, and so being taken into the stomach of the animal. Hence the stomach, bowels, and kidneys are seen to become the primary seats of the disease. The rains, which might be supposed capable of washing away and totally dissipating this poison, but served to render it more quickly fatal. Only the hard frosts which appeared late in autumn could effectually destroy it. Until then the very grass seerned alive with the plague. 
Symptoms. - The first indication of the presence of this disease consisted in increased heat of the body, as shown by the thermometer. The natural heat is about 100 。 Fahrenheit; the temperature in those first beginning to be affected would be $102^{\circ}$ or $103^{\circ}$, while in the more advanced stages of the disease, it rose as high as $106^{\circ}$ or $107^{\circ}$. And even the internal organs, immediately after the beast was slaughtered, would carry the mercury up to $106^{\circ}$. This rernarkable and uniform augmentation of temperature became an invaluable prognostic of the onset of the disease; since it could be found three or even five days before the appearance of the more obvious external symptoms. And this interval admitted of the timely administration of medicines calculated to antidote the poison in the system, even before it had fully developed itself.

The period of incubation was very short; in some instances native cattle have died within four or five days of being exposed to the infection. But the rapidity of the course of this disorder may depend somewhat upon the amount and intensity of the poison absorbed into the system. In other instances the native cattle began to be sick about two weeks after some Texas droves had remained over night in their vicinity.

In the beginning of the disease there is an uncertainty of step and trembling, showing an inability to remain firmly standing on the feet, and with this a disinclination to move; the head drooping. The appetite does not seem to be impaired, nor is there any unusual thirst ; the skin is alternately hot and cold; the secretion of milk is diminished; and about the fourth or fifth day a marked change in the appearance of the animal takes 
place. The abdominal walls are shrunken, the animals becoming lean, breathing quick and short, and do not have strength enough to raise themselves, and when raised cannot remain in that position for any length of time. The secretion of milk decreases daily, the secretion itself continuing until death, and is of a thick, creamy character. The secretion of urine is also changed, the animal having a constant desire to urinate, succeeding, however, in ejecting only a small quantity of bloody urine. The fæces are discharged with great effort, and are dry and hard. It is not easy to separate into any distinct stages the course of a disorder which so rapidly reaches its conclusion; but we give successive pictures.

In the more advanced progress of the disease, the animals stand apart from their fellows; present a listless appearance; the head hanging low down; the base of the horns hot; the ears drooping; the eyes dull and staring; the spine, or back, peculiarly arched; the hind feet being drawn under the body and placed in a bracing attitude; a tremulous creeping over the flank muscles, with frequent efforts at voiding fæces, which are generally small, hard, and rounded, and covered with bloody mucus. In the further stages of the disease, there may be diarrhœa, and frequent passing of urine, which has a dark and bloody appearance. A fully developed case presented the following symptoms: An arched or roached back; head carried low down; ears drooping; eyes staring, with a dull, glassy appearance; gait tremulous, and staggering in the hind quarters; the freces hard and streaked with blood; urine copious, and bloody in appearance ; pulse about eighty ; respiration, forty in a 
minute; the temperature of the rectum, as shown by the thermometer, was $107^{\circ}$ Fahrenheit. The debility becomes so great, with refusal to eat, that the animal lies down, and is unable to rise; death closes the scene.

The rapidity with which, after its period of incubation, this disease advances may be judged from the following statement of the condition of a steer sick only thirty-two hours: Temperature of rectum, $107 \frac{3}{4}^{\circ}$ Fahr. ; respiration, 36 ; pulse, 76 ; from nostrils, profuse mucous discharge, streaked with blood; anus appears dry and contracted; fæces almost natural : urine bloody ; the animal balances himself by strongly twisting his head and neck to the left. The condition of an ox in the last stage of the disease, and unable to rise, is thus briefly stated: Head drooping near the ground; eyes staring, dull; horns cold; body hot; breathing rapid; pulse feeble, unable to count it; coat rough, flies adherent; temperature of rectum, $106 \frac{1}{2}^{\circ} \mathrm{Fahr}$. In another case, the temperature of the blood flowing from the aorta as it was slaughtered was $107^{\circ} \mathrm{Fahr}$. The following description of another sick animal,also far advanced, will complete our picture, from which the reader will have no difficulty in recognizing the disorder, when the first case presents itself before him: A four-year-old steer, standing with head low down; saliva running from the mouth; ears drooping; eyes staring, glassy, and dull; coat rough ; arched spine; hind feet drawn under the body; voiding bloody urine; fæces fluid and dark; gait staggering; trembling (subsultus) of a portion of the flank muscles; great debility; unable to walk any distance without falling down; rising with great difficulty; temperature of rectum, $105 \frac{1}{2}^{\circ} \mathrm{Fahr}$. 
Upon milch cows the general effect of this disorder is the same as upon other stock; in most cases the flow of milk is instantly stopped. In two cases, which came under the observation of Mr. Atkins, a leading agriculturist of Illinois, of cows with calf, the disease culminated in abortion, which was followed by immediate convalescence of both animals. Milch cows seem to be very susceptible to the disease. Calves are not so. Not a single instance, says Mr. Atkins, have I known of a calf dying of the disease.

When dead animals are examined, even three or four hours after death has taken place naturally from this disorder, it is found that putrefactive decomposition has already begun. And in some instances it was found in the dissection which instantly followed the slaughter of condemned animals, that putrescent disorganization was already in progress in the kidneys, spleen, and liver, and the capillary blood vessels of these organs and the surrounding tissues were already filled with gases resulting from general decomposition.* From the very nature of the malignant virus, which thus begins to putrify the viscera even before life is extinct, it is evident that the disease itself-the obvious results of this virus-must run a very rapid course. In some recorded cases death occurred in four days from the first appearance of sickness; in one in particular, the sickness set in ten days after exposure, and the animal died in a few hours. Thus it has been considered by some a fever of but one

\footnotetext{
*The following statement of the condition of the internal organs of an animal examined immediately after death shows that he died of putrid blood-poison: "Liver enlarged, softened; weight, $16 \frac{1}{2}$ pounds; gall very thick, dark; bladder one quart of urine, dark, bloody ; kidneys perfectly disorganized ; rectum gangrenous ; spleen an enlarged, engorged, pulpy mass."
} 
paroxysm. This last access of fever, which marks the sudden close of the animal's life, is ushered in by a chill. And from this and many other observed analogies, as well as from its far Southern origin, this disorder has been regarded as corresponding to the yellow fever in man. Indeed it is by Dr. Stites affirmed that the Texas cattle disease may properly be termed the yellow fever in cattle. But it is doubtful if the yellow fever in man possesses a virus so malignant, infectious, and contagious as this cattle plague does. This latter disease may be communicated to horses; and an account is given by Mr. Hill, of Tolono, Illinois, of the prevalence of this Texas epizootic among the horses of the same district in which the cattle had been swept away before the horses began to die. In some cases, the disease had been complicated with pleuro-pneumonia. What is quite singular, calves appear to remain exempt from this disease.

Pathology.-The liver presents, upon examination after death, an increase in size and weight, and shows sometimes a "waxy" condition, especially in animals that had suffered long with the disease. Sometimes there appeared a similarity to that peculiar "dry" condition which occurs in long protracted fatal cases of yellow fever in man. The spleen also is very much enlarged, no doubt from the necessity laid upon it to become the temporary "waste-gate to the portal and gastric circulation," in consequence of the engorgements and obstructions in the circulation of the liver. The kidneys would likewise be enlarged, dark-colored, and engorged throughout with dark blood. Their functional action was kept up almost to the last in the desperate effort of Nature to free herself from this disorder; hence the dark bloody urine 
which characterizes this disease, and with many people gives its distinguishing name of "black water." The kidneys, of course, became themselves destructively affected by the poison they attempted to eliminate from the blood, and presented on dissection evidences of that "marvellous rapidity of disorganization and putrefaction" to which we have already referred as attacking the abdominal viscera even before death.

\section{Treatment.}

The American rinderpest bears a striking resemblance to that already described as prevalent in Europe at various periods for many centuries, and it especially corresponds to the form so lately raging in Great Britain. The more common homœopathic preparations which then proved so successful, as well in Holland as in England, would no doubt be applicable to such a variety of this disorder as appeared in the United States. But there is another remedy, Carbolic acid, which is no less truly homøopathic to this complaint, and which, both as a preventive and as a remedial measure, leaves little to be desired. This most powerful antiseptic, given in large doses, destroys all ths normal vital germs in the blood, and has been known thus to produce death in three minutes.* In smaller doses, Carbolic acid has the wonderful faculty of attacking and destroying all the abnor-

*Carbolic acid "acts by attacking vitality in some mysterious way. The powerful action which this drug exerts on the phenomena of life is the most remarkable property which it possesses. In the presence of Carbolic acid the development of embryotic life is impossible, and before its powerful influence all minute forms of animal life must inevitably perish." $-W m$. Crookes, F. R. S., "On the Application of Disinfectants in Arresting the spread of the Cattle Plague." London, 1867. 
mal or diseased living germs in the blood, and thus it acts internally as an antiseptic in the same manner that it does externally. Hence this drug becomes a homøopathic agent seemingly prepared for this very class of infectious virulent disorders, which yield with so much difficulty to any other medicines, since, in small doses, it saves life (by destroying abnormal or infectious germs) in exactly the same manner that in larger doses it destroys life by destroying all the vital germs.

The treatment of the cattle plague in America may be divided into two stages: one preventive or prophylac. tic, the other curative. As the New York State Commissioners observe in the conclusion of their report: "With reasonable care on the part of stock-owners in keeping themselves supplied with Carbolic acid in some one or more of its forms, and using it freely on their premises, there appears to be a perfect immunity from diseases that have, hitherto, carried inevitable destruction wherever they appeared.

"Further than this, the observations of the Commission warrant the belief that this same agent possesses curative properties of the greatest value, when applied to 'foot-rot' in sneep.

"From the fact that Carbolic Acid acts specifically upon all germs or seeds of disease that are propagated in a manner similar to the spores or fungous parasites of the Texan disease, it is not too much to hope that it may be used successfully in the treatment of many diseases in animals heretofore regarded as incurable, especially the glanders in horses; inasmuch as the recent researches of the world-renowned Hallier, or Jena, have brought to light in the nasal discharges and circulating blood of 
glandered horses the Coniothecium equinum, a microscopic parasite of the same genus as the Coniothecium stilesianum, which is the active agent in the Texas cattle disease."*

"Disinfection and Disinfectants.-Carbolic Acid is an absolute and perfect disinfectant. It not only destroys the odor, but kills the virus of the disease. We advise all farmers and drovers, who have reason to suspect that their cattle have been exposed to the infection, to sprinkle the substance known as 'heavy oil,' which contains about ten per cent. of Carbolic Acid, abundantly about the yards where they are confined, and to put some Carbolic Acid into the water they drink in the proportion of one part of pure Acid, with thrice its own weight of sal soda, to one thousand parts of pure water."

For medical treatment the following method has proved very successful, as may be judged from a single statement. Out of a herd of thirty head, eighteen were diseased; nine of these died before any treatment was instituted, the other nine were saved in the following manner: "The sick animals were placed in a small inclosure by themselves. Pure Carbolic Acid was placed in a large, open-mouthed bottle, dissolved in water. This was held to the nostrils, and given by inhalation at short and repeated intervals. The heavy oil of coal tar, containing seventy per cent. of Carbolic Acid, was liberally sprinkled upon the yard where they were kept, thus presenting the fumes of the Carbolic Acid constantly.

* Annual Report of New York State Agricultural Society, 1867, Part II, p. 974. By reference to the section on "Glanders," it will be seen that the anticipation above given has been confirmed. 
The feeding was low diet, plenty of water, salt, and outdoor air."*

Carbolic Acid seems to exert the same direct, specific influence in destroying the noxious, virulent germs of the infection of glanders and the cattle plague that camphor does in antidoting strychnine, or Belladonna in antidoting poisonous doses of Opium. Carbolic Acid may, indeed, have been first introduced into medical practice by the allopaths, but it is none the less strictly homœopathic in its remedial action, and is very properly given by homœopaths, even in considerable doses. But the method of administering this by inhalation, which old school veterinarians have found most efficacious, is the very one for which Hahnemann, the founder of homoopathy, has been subjected to such unsparing ridicule.

\section{6.-Cow-pox.}

Cow-pox is of two kinds : the milder form is Spurious Cow-pox, and the more malignant form is True Cowpox.

Contagion is certainly one very important and perceptible cause of this disease, but the remote causes have hitherto remained very obscure. It is to be believed, however, that atmospheric influences, combined with mischievous food, would generate it in its original and specific character.

Symptoms of Spurious Cow-pox.-The appearance of a

* New York State Agricultural Society Report, 1867, Part II, p. 1025. 
pustular eruption on the teats, the elevated spots being without any uniformity of size or shape, and attended or unattended with slight constitutional disturbance; after having become developed, the crown becoming distended with thick, opaque, yellowish matter, the exudation produces a crust, which, if undisturbed, will fall off, of its own accord, leaving the skin uninjured, but which, if artificially removed, either in milking or otherwise, will leave behind it a multiplicity of diminutive, ulcerative sores, more or less sluggish, and slow to heal.

Symptoms of Specific Cow.pox.-It is preceded and attended with considerable manifestation of fever. The constitutional disturbance often runs high; the milk fails; the appetite is impaired, and rumination is suspended. The eruption, like that just described, presents itself on the teats, sometimes extending to adjacent parts ; it is, however, of more gradual development, and the pustules are more extended, more uniform (being round in shape), surrounded with a bright red, inflammatory circumference, and becoming concave at the apex, with elevated margins; the pustules, in this instance, first exhibit the presence of a thin, serous fluid, which gradually thickens, and becomes yellower; oozing and scabs ensue, subject to the same conditions as the former, but which, if disturbed, leave behind them a severe, deep-seated, and often malignant ulceration.

\section{Treatment.}

Where any treatment is requisite, it must be similar to that advised for small-pox or variola itself - which see; also bathe the pustules with German Wash, and give internally Antimonium crudum and Thuja occidentalis. 


\section{7.-Fractures.}

Fractures are distinguished by some or all of the following symptoms: 1st. The bone is displaced. Pressure or weight thrown on it causes still further displace. ment. In the case of long bones the broken ends, if the fracture is right across, may pass each other, and thus the limb will be shortened.

$2 d$. The fractured limb partially or entirely loses its power of voluntary movement; but by external force it can be moved more readily and in a greater variety of directions than when sound.

$3 \mathrm{~d}$. When a broken bone is thus moved, the fractured surfaces may be felt and heard to grate on each other or "crepitate." This crepitation is especially noticeable, if the fractured surfaces are rugged, and still more in comminuted fractures. In fractures of an important bone there will also generally be twitching of the neighboring muscles.

4th. From rupture of the arjacent bloodvessels and consequent escape of blood, or from laceration of the neighboring soft parts, there is usually a considerable swelling about a broken bone. From the same causes the skin, where it is visible, may be discolored, and the parts hot and tender.

The causes of fracture may be included under two heads, namely, violence to the bone itself or to some adjacent part, such as occurs in the case of falls or kicks; and less commonly from excessive muscular strain. The sesamoid bones, for instance, are occasionally broken by the stress thrown on them by the suspensory ligament.

In some horses, as in some men, the bones appear to 
be abnormally fragile, probably from imperfect nutrition and fatty degeneration. The liability to fracture also increases greatly in old age.

Fracture of the Peivis.-Any of the three bones constituting the pelvis are liable to be broken. The most common and least serious of these injuries is chipping a piece off the spinous process of the ilium, usually from the animal coming in forcible contact with a doorpost or gate. When the observer stands behind, the flatness of the injured side is very perceptible, and in popular language, the hip is said to be "down." The piece of bone, when separated, occasions no inconvenience, and after a time becomes inclosed in a cyst. A similar injury sometimes, though much more rarely, occurs to the spinous process of the ischium in the posterior part of the hip. These injuries do not usually cause any permanent unsoundness.

The shaft of the ilium may be broken by a fall. This accident most frequently occurs in heavy draught horses. On account of the stoutness of the periosteum and the general position of the bone, the broken ends may not be much displaced; but the nature of the accident is apparent from the tenderness of the adjacent parts, and from the swelling and soreness felt, when the hand is introduced by the rectum, and also by the crepitation observable especially when the patient is moved slowly forward, whilst the hand within the rectum is held over the seat of injury. The animal will straddle greatly in his gait.

If the fracture is not extensive and does not involve the hip joint, and there is not much displacement, and the animal is not irritable, repair may take place. The 
patient must be restrained from laying down, and kept as quiet as possible. Nature may and often does in these cases keep the broken parts in proper apposition, but art cannot, from the position of the parts, do anything to assist. In favorable cases the bones will have fairly reunited in about three months, and the animal may probably be sound.

Occasionally the fracture is very extensive and involves the hip joint. More rarely the body of the ischium is broken. In a few cases the symphysis pubis is fractured. All such injuries preclude any hope of recovery.

Fracture of the Pastern Bones. - Fracture of the great pastern bone sometimes occurs, but that of the small pastern or os coronæ is more frequent. It occurs very suddenly, and particularly in horses with high action. Probably the fracture results from the foot coming to the ground in a faulty position, and thus receiving unexpected concussion, whilst the parts of the leg are not in the proper position to receive it.

Fractures of either of these bones are very uncertain as regards the chance of cure. When the horse gets a little better, he is very apt to attempt to move his leg or to place weight on the foot, and then the fractured ends may probably separate again. A simple longitudinal fracture is worth treatment, but a comminuted fracture is not.

BROKEN RIBS. - The ribs are rather frequently broken; and if so, the ends generally overlap. It may be possible by manipulation temporarily to readjust the ends, but it is not possible by any mechanical means to retain them in their proper position. No material harm ordi 
narily results from their overlapping; though in some cases a broken end thoroughly displaced and turning inwards may injure some of the internal organs, and even cause a fatal result. To prevent this, excision of the part may in such cases be necessary. Nature will afterwards fill up the intervening space with callus.

The treatment, in addition to rest, consists in apply ing a large wide roller or stout webbing over the part, in order to confine the bones in one position as much as possible, and thus prevent undue expansion of the ribs. The roller must be kept in its place by means of straps attached to a collar on the neck.

Fracture of the Nasal Bones. - Fracture generally produces depression of the bones, and the breathing is then interfered with. The treatment consists in raising the bones with some blunt-pointed instrument to their proper position. To do this it will probably be necessary to make an incision through the skin. The bones must be retained in position, until reunion takes place, by pitch plaster and strips of stout pasteboard, or by any other mechanical contrivance convenient in the particular case. The animal's head should be kept tied up for a considerable time afterwards.

Fracture of the JAWS. - The lower jaw is liable to be fractured, especially under the molar teeth, where it is very slender. After readjustment, splints and plasters must be applied to retain the bone in its place; and until union has taken place, the animal must be fed on sloppy diet, so that he may require to masticate as little as possible.

Some excellent remarks on facial injuries by Professor Varnell will be found in the Veterinarian, 1866-1867. 
Partial fracture of the Tibia. - If the tibia be fractured right across, the case is hopeless; but when the blow occurs on the outside, where the parts are well protected by muscles and ligamentous and tendinous tissues, it often happens that the bone is not fractured through ; or the fracture may be merely a longitudinal split. In such cases the periosteum may be strong enough to hold the parts together; and if the nature of the injury is discovered at once, the animal will often recover after a few weeks' rest. It very often, however, happens that the real nature of the injury is not suspected, and the horse after a few days' rest is again sent to work, and then the bone at the first strong exertion becomes fractured through.

Fracture of the Shoulder-Blade.-This accident is rare. In walking, an animal trails the toe along the ground. If, when the foot is brought forward, the hand be placed on the shoulder, a crepitation will probably be felt.

The fracture is usually across the neck; and if so the case is hopeless. A longitudinal, but not a transverse, fracture in any other part may possibly with rest reunite evenly, and the animal may perhaps become sound.

\section{Treatment.}

The first indication is of course to set the fracture, to replace the parts, if out of place, in their natural position; the homøopathic remedies for the cure of the fractures are few in number, but sufficiently efficacious.

Arnica, externally and internally, as recommended for bruises, should first be employed when the fracture is caused or occasioned by bruises or contusions. 
Symphytum should be employed afterwards, and from the first in other cases, in accordance with the following directions of Gunther :

In horses.-It sometimes happens from a fall or a severe blow, that a greater or less portion of the bones of the ilium become fractured. There then appears in the same place a hot, painful tumor; the horse limps, chiefly at the commencement; and when we view him from behind, we see the affected haunch lower than the other. This accident is never dangerous in itself.Every time the case is presented to me, I have removed it by employing externally the strong tincture of Symphytum. I also give some drops of this internally from time to time.

Fractures of the ribs are often cured of themselves; they are treated with Symphytum. When they are complicated with splinters projecting internally, they are liable to produce suppuration of the lung.

Like other fractures, those of the bones of the nose are cured in a little time by Symphytum. Any splinters that may exist must be carefully removed.

Fractures of the bones of the legs are not uncommon in the horse. They are discovered by the animal being unable to rest on the affected limb, which, when carefully examined, exhibits the presence of flexion in a part where there is no joint, and causes a crepitation which is produced by friction of the ends of the bone. An inflammatory swelling soon attacks the part, which becomes very painful to the touch. Fractures of the limbs have been considered as incurable, in consequence of the weight of the body; but several facts have satisfied me that with proper precautions we may suceed in 
curing them. The first is, after having duly fitted the ends of the bones as exactly as possible, to surround the fracture with broad bandages of cloth, over which we are to apply two iron splints, excavated in the form of a gutter, so that the one placed on the posterior surface may pass some inches beyond the hoof, and the affected limb may rest on it. We must then pass large girths around the chest and quarters, and under the belly an empty sack, or broad piece of canvas, which is attached to the ceiling with ropes and pulleys, to be altered at pleasure, so that during the entire time of treatment the animal may be kept in a state of semi-suspension.With respect to internal treatment, he is to take on the first day two doses of Arnica, then one every other day; then after four or five days, every two days only, one dose of Symphytum; and the bandage is to be frequently wet with cold water, to which there has been added from a third to a sixth of this medicine, in the mother tincture. At the end of eight days the bandage must be removed to see whether the fragments of the bone have been duly brought into apposition, after which it is reapplied, and so left until there is a complete cure. Up to this time we may continue to use the Symphytum, both internally and externally.

Calcarea phosphorica may be substituted, in twenty-drop doses, twice daily, where there is too great delay in the bones uniting; where, instead of thus firmly growing together, a callus remains and threatens to result in a movable or false joint.

In oxen.-It is not an uncommon occurrence for oxen to break a horn ; the result is violent hemorrhage, which is to be stopped by fomentations with Arnica water.- 
Sometimes we succeed in restoring the horn by immediately fixing it to its place, tying the animal by itself to a ring, so that it may not rub against anything, and administering internally, first, Arnica, then, in a little time after, Symphytum, alternately with Squilla. But, generally speaking, we cannot succeed, especially when the horn has become cold. We then envelop the stump in linen cloths soaked in Arnica water, which are to be renewed frequently, and we should make the animal take internally every two days a dose of Amica, or one of Symphytum, if the bone also has been fractured. We are told that a double dose of Squilla has also been found very useful in such cases. The cure is effected with great facility. Oxen frequently fracture the ossa ilium, an accident which rarely occasions fatal consequences, and in which Symphytum should be employed, both internally and externally. If there be much heat, inflammation and swelling, some doses of Aconitum and Arnica may be administered with advantage.

\section{8.-Conformation of the Hock.}

A good-shaped hock is seldom unsound, whilst one of defective conformation generally becomes diseased, if exposed to hard work.

To know a good from a bad, a sound from an unsound hock, requires some time, trouble, and attention, but not more than it is worth any horseman's while to give. It is good practice to get the bones of a hock, to put them together, to examine the formation of each, and to feel them when placed in their natural position; and then to manipulate and compare with them the 
prominences of the bones in the living animal, especially on the seat of spavin. With a knowledge of conformation derived from handling the bones, the satisfactory examination of the hock becomes easy.

The hock consists of six bones, namely, the astragalus, cuneiform magnum, cuneiform medium, cuneiform parvum, cuboid, and os calcis. The first five of these may be distinguished as weight-bearing bones, whilst the os calcis or bone at the back acts as a lever to the tendons of the leg.

The true hock joint, however, is formed by only two bones, namely, the tibia, or upper bone of the leg, and the astragalus. The other bones, though they possess a limited amount of motion between each other, do not enter into the true hock joint.

Peculiarities of a good hock. - The outline should be clean, rigid, and, in an adult horse, well defined. Any puffiness or swelling is a sign of weakness or disease.

The bones should be large and prominent. Large size is essential to strength, and prominence is necessary in order to afford due leverage and attachment to the tendons and ligaments. Large bones are usually accompanied by large and well-developed tendons and ligaments.

The hock, when viewed from the side, should appear wide both above and below. Strength and size both of bones and ligaments are indicated by lateral width.

The hocks should be neither straight, nor over much bent. Undue concussion results from the former, whilst weakness and liability to sprain accompany the latter formation. If the hocks are placed too far behind, there will be a want of propelling and jumping power.

The hocks should be placed directly under the centre 
of gravity. Any deviation from the perpendicular line laterally, as is the case when the hocks are inclined too much in or too much out, is a source of weakness and therefore of disease.

In order to give due leverage to the muscles of the thigh, it is essential that the tibia should run down well into the hock. A well-developed and prominent os calcis is also essential to the leverage of the tendons of the legs.

Badly-formed hocks are marked by peculiarities of conformation the reverse of those we have just described.

The diseases found in the hock generally correspond pretty closely with the points in which the conformation is defective. For instance, in upright hocks we may expect bog-spavin and thorough-pin; in over-bent hocks, curb; in narrow hocks, spavin and curb may be looked for; in hocks which bow out, thorough-pin; whilst in small hocks there is general want of strength and consequent liability to sprain and disease of any sort.

It is not, however, to be supposed that horses with defective conformation either in the hocks or elsewhere are useless for any purpose. All that we intend to say is that defective conformation entails a special liability to disease. Again, horses which, from defects of conformation, may be unfit for one sort of work, may be available for another. A horse, for example, whose hocks will not stand the violent exertion of hunting, may last for years for quiet riding or harness work.

\section{9.-Spavin, Bone Spavin.}

Spavin is an exostosis in the region of the hock. It is usually found to involve two or more of the weight- 
bearing bones. The inner small metatarsal bone of the leg, is sometimes, though but seldom, involved.

The conformation of the hock generally has been dwelt upon in the preceding section. The true hock joint consists in the articulation of the tibia and astragalus. This joint is never primarily, and but seldom even ultimately, except as a result of open joint, affected by exostosis.

But besides the true hock joint, there are also joints with a limited amount of motion between each of the other bones, which make up the structure of the hock. The exostosis known as spavin generally forms between two or more of these bones and interferes with their gliding motion; or it may form on the outside of the bones, or partly on the outside and partly between the bones. As stated above, it very rarely extends to or irnplicates the bones of the true hock joint.

When from undue concussion, pressure, sprain, or such like causes, irritation is set up either in or in the neighborhood of the above mentioned bones, it is probable that the irritation will be quickly followed by inflammation, more or less severe, according to circumstances, of the periosteum and articular surfaces of the bones implicated. Irflammation will, as usual, probably be followed by effusion from the overloaded blood vessels of the part. In due time the watery parts of the effusion will be absorbed, and the remainder will solidify. In accordance with the usual rule of nature, the deposition will partake of the character of the part into which it is effused. In short, it becomes bone, or in other words, a spavin is formed.

The probability of the occurrence of spavin in any 
particular hock is mainly dependent on its conformation. If the hock is large and strong, and its position is good and true as regards the incidence of the superincumbent weight, it is not probable that it will be injuriously affected either by concussion, weight, or sprain. But if there is any defect, either in the conformation, structure, or position, it will probably suffer in some part or other; and at that point, sooner or later, accord ing to the circumstances and nature of the work to which the animal is subjected, we are likely to find disease.

It is impossible to define accurately the position of spavin. It varies in some degree, according as weak or defective structure, or improper position of the hind legs, or incidence of the line of weight, or sprain of the ligaments, or such like causes, may induce pressure or concussion on any particular part. The greatest pressure and concussion, however, are always felt towards the inner side, and hence spavin is always found somewhere on that side. The most common seat is between and towards the front of the cuneiform bones.

Spavins may occur on the exterior of the bones. In such cases they are easily seen and felt, and hence are called "detectable;" or they may occur between the bones, in which case there may be little or no external enlargement. These are termed "occult" spavins. During the formation, however, of the exostosis, some degree of extra heat and tenderness on pressure may generally be detected. After consolidation, the existence of occult spavins in many cases can only be divined by . the nature of the lameness they produce.

As a general rule, exostoses on the exterior of the bones arise from sprains of the ligaments of the hock, 
whilst those between the bones are produced by pressure and concussion.

The importance of a spavin depends on its position, rather than on its size.

If it is situated between the bones, and especially if towards the front, there is always great reason to fear that it may, even though small, or very small, interfere with the gliding motion of the weight-bearing bones. Further, any such exostosis, however slight, is likely, on account of its interfering with that motion, to cause further irritation and inflammation in the part, and ultimately ulcerative disease of the articular cartilages of the bones.

If, on the other hand, the spavin, even though it be between the bones, is placed far back, it is of less consequence, because there is not much gliding motion in the posterior portion of the hock; and therefore the exostosis, even though large, may not occasion much inconvenience. Indeed, in many old horses we find that positive union has taken place between the posterior portions of the bones, without ever having caused any sensible lameness.

Spavins on the external surfaces of the bones, and not between them, are less serious. They may or may not interfere with the action, according to their size and position in each particular case. Spavins placed posteriorly, and not between the bones, even though large, seldom interfere much with the free motion of the hock.

In well-developed cases the lameness arising from spavin is very peculiar and characteristic. It is caused by imperfect flexion of the hock, and in consequence, the toe, instead of being properly raised, is dragged along 
the ground. Towards the end of the month the toe of the shoe is sensibly worn by this abnormal friction.

In slighter cases some stiffness of the hock and an occasional tripping of the toe may be noticed, and also a sort of vibration in the hock, when the toe comes to the ground. These peculiarities will be most observable if the horse is trotted on hard, smooth ground. The animal should be especially watched in turning, when a certain degree of flinching will be detected. Exercise, even for a few minutes, greatly diminishes the symptoms; but when the horse, after exercise, is allowed to stand till cool, the stiffness will recur, probably in an increased degree.

In the stable a horse, though only very slightly lame from spavin, will often drop very much, if made to move in his stall to one side, when the weight comes on the diseased leg. In bad cases, in a state of rest, he will usually keep the leg flexed.

For treatment, refer to recipes in Appendix.

\section{0.--Splint.}

As splint, like spavin, generally arises from or at least is connected with some structural weakness, we propose in the first place briefly to consider the conformation of the fore leg between the knee and the fetlock.

The fore leg immediately below the knee is made up, as regards its osseous structures, of three bones, namely, the great metacarpal or shank, or cannon bone, and two smaller bones, called the splint or small metacarpals.

These bones are not united together, but each of the small bones is attached to the great or shank bone by 
interosseous ligaments. The object of this sort of attachment appears to be to allow a very limited power of motion or elasticity between the bones. A similar mode of attachment is found in other parts of the body, where a certain degree of elasticity is required, as, for instance, between the bodies of the vertebræ.

In the fore leg the elasticity conferred by this peculiarity of conformation is useful in diminishing concussion. But this sort of attachment, though useful for the above purpose, gives less strength than union of the bones.

Splint is an exostosis or deposit of bone either between one or other of the small bones and the shank, or upon any of the three bones. The cause of the abnormal growth is irritation and inflammation either in the interosseous ligaments, or in the periosteum, or in the bones themselves.

As the greatest strain and concussion always fall on the inside, on account of its being more under the center of the superincumbent weight, we generally find the exostosis on or towards the inner side - except in horses which turn their toes in, and thereby throw the greater weight on the outer side.

Splints, for the purpose of explanation, are sometimes divided into two classes, namely, those which arise from sprain and inflammation of the interosseous ligaments; and those which arise from irritation and inflammation of the periosteum, or of the bone itself. But for practical purposes it is not necessry to maintain this distinction. Indeed, whichever structure be primarily affected, the other also generally becomes more or less involved.

When inflammation is set up in a bone, or in its periosteum, an exostosis is likely to be the result, unless the 
action is checked in a very early stage. The mode in which osseous material is effused and consolidated was explained on a previous page. The exostosis or "splint" will probably unite firmly the small to the great metacarpal bone.

Again, if the inflammation is set up in the interosseous ligaments, it generally results in absorption of the original tissue to a greater or less degree, according to . circumstances; and in lieu thereof osseous material is deposited, which, when in due time consolidated, unites together the bones between which it is effused. The cause of this peculiar effect of inflammation on fibrocartilage, of which tissue the interosseous ligaments are mainly composed, is not well ascertained; but its almost invariable occurrence is a well-known fact.

Though the immediate causes of splint are irritation and inflammation in the parts affected, yet the occurrence of these causes in any particular horse and at any particular time, is mainly dependent on the conformation of the leg, on the work to which the animal is subjected, on the weight he has to carry, and on his age.

If the bones of the leg are small, or if not positively so, are yet small in reference to the carcass, or if there is undue length between the knee and the fetlock, or if the ligaments and tendons are small, or if the legs are crooked, we may be pretty sure that such defects of conformation will probably give rise to irritation and inflammation in the weakest part.

Again, if the pasterns are overlong, there will be undue stress on the parts above; or if, on the other hand, they are very short, there will be excessive con . cussion. These effects, though arising from defective 
conformation below, may nevertheless be felt above, notwithstanding the portion of the leg from the fetlock to the knee may be well formed.

Again, if the legs are not placed well and directly under the center of gravity, or if the incidence of the weight of the body does not fall fair and true on the legs, there will be irritation and inflammation in the part unduly pressed upon.

But no cause of splint is perhaps more common than the ordinary practice of subjecting young horses to work, for which, however good their make and shape may be, their young bones, ligaments, and tendons are unequal. Farmers, who breed horses, generally put them to harrow at two years old, they often ride or drive them at three, and hunting men sometimes expect four and always five year old animals to carry them across country.

Horses at an early age may no doubt do a certain amount of work, and perhaps may be none the worse for it ; but the work demanded of them is often in excess of the age and capability of the animal. It is mainly from this cause, as we think, that we see so many horses, whose make and shape are unexceptionable, affected with splints.

The exostosis arising from these and such like causes usually appears about midway between the knee and the fetlock, because the middle is the weakest part in long bones. The reason why it appears on the inside rather than on the outside was explained above on page 382 .

Defects of conformation are, however, we must remind the reader, in a great degree relative to the work which we require from a horse. Many an animal's legs, for 
instance, which might stand for years for harness work, may be battered to pieces in a short time by hard riding along a road, or strained by hunting in a deep country.

Though some defect in conformation, or some excess of work relatively to age and structure are the ordinary causes of splint, yet in some cases the exostosis can be traced to no other causes than an hereditary predisposition to throw out ossific material. In such animals we generally find spavins and other exostosis concurrently with splint.

Exostoses, not true splints as defined above, are sometimes found on the outside of the leg, wholly unconnected with any of the above causes, which arise from inflammation set up in the bone or periosteum from the effect of a blow, such as a servant may give a horse with the handle of a pitchfork, or the animal may give himself accidentally, in the bunting field.

In most cases the splint, for reasons already given, appears on the inside and a little above the center of the bone, between the knee and the fetlock. Special circum. stances, however, in some degree vary its position. If, for instance, the incidence of the weight does not fall true on the legs, the exostosis will probably form on that part of the bone or bones on which undue pressure comes; or if the leg is crooked, the exostosis will be found at that spot where the malformation causes unusual strain.

Splints, when fully formed and consolidated, do not of themselves, as a rule, cause lameness. They no doubt lessen to a certain degree the elasticity of the tread, but they do not affect the action in any perceptible degree.

The importance of a splint is dependent more on its $17 *$ 
position in reference to the action of the other leg, or to the passage of the tendons and suspensory ligament, than on its size. If it be so placed that its protuberance is likely to be struck or interfered with by the other leg in action, splint becomes a serious evil. If it is not so placed, the mere exostosis may be of little consequence. This question can only be settled by actual experience in the particular horse affected. A splint in one horse, from some peculiarity of action, may be interfered with by the movement of the other leg, whilst one in an exactly similar position in another horse may not suffer.

For treatment, refer to recipes in Appendix.

\section{1.-Ringbone.}

Ringbone is an exostosis, either on the upper or on the lower pastern bone, affecting in the one case the upper pastern joint, or in the other case the lower pastern or coffin joint, around the coronet.

Ringbone more frequently affects the hind than the fore fetlocks. The degree of lameness is much greater in the lower than in the upper disease.

False ringbone is an exostosis on the bodies of either of the above bones, not affecting or interfering with the joint.

Ringbone is generally connected either with weakness and consequent sprain of the fibres of the lower divisions of the suspensory ligament, which are inserted into the anterior part of the coronet bone; or with sprain of the articular ligaments of that bone; or it may arise from concussion, or from a blow, tread, or other wound, or from any cause producing undue or unusual strain on 


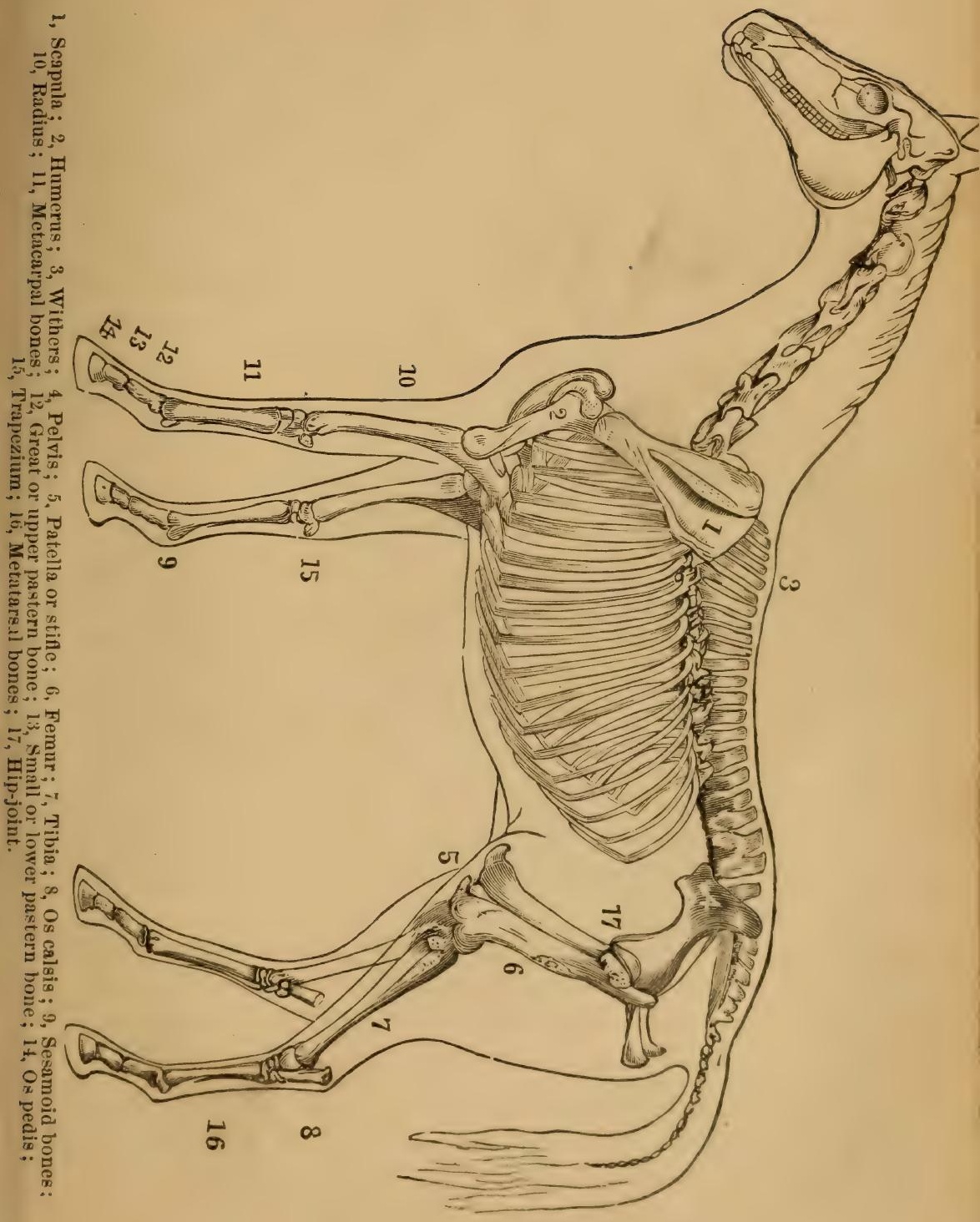



the ligaments of or about the fetlock. From any of these or such like causes inflammation may be set up in one or other or in both pasterns, and an ossific deposit may be the result. In some cases a predisposition to this disease appears to be hereditary.

Ringbone is common in horses with long pasterns, where there is necessarily a tendency to weakness; and also in animals with unduly short or upright pasterns, in which formation there is a tendency to excessive concussion.

Lameness arising from ringbone (as is usually the case when an osseous structure is affected) is more perceptible on hard than on soft ground. The special peculiarity to be noticed is some stiffness or want of flexion in the fetlock joint, and a consequent snatching up of the foot in action. Some swelling and heat are also, in most cases, even in the early stage, apparent about the fetlock, and in a later stage increased heat will invariably be detected.

Ringbone, when fully formed and consolidated, will produce lameness or not, according to the extent, and still more according to the position of the exostosis. If it is so placed as to interfere with the action of the joint, the horse will probably be incurably lame. In slight cases no further mischief occurs than some diminution of the elasticity of the tread. We must, however, warn the reader, that with the renewal of severe work, inflammation is apt to be again set up, accompanied with a fresh deposition of bone.

The formation of ringbone, especially if it appears in more than one fetlock, is generally a sign of weakness or defective formation; and therefore an intending pur. 
chaser will do well to think twice before he buys a horse so affected, if he wants him for hard or fast work. But when the exostosis is found on only one fetlock, there is a probability that the inflammation giving rise to the ossific deposit may have originated in a blow or tread, or some such accidental cause.

For treatment, refer to recipes in Appendix.

\section{2.-Contraction.}

Contraction may be the result of a natural tendency, accelerated by the effect of shoeing; of neglect in the sufficiently frequent repetition of shoeing (frequent removal being highly beneficial when the hoof is evidently predisposed to contraction) ; excess of paring, especially as regards the bars; slow inflammation of the fleshy parts and coatings of the bone adjacent to the horny surface; neglect in providing moisture (by means of the pad) to the sole when in the stable; irregular and deficient exercise; with rich and excessive feeding, and a superfluity of litter, allowed to remain in the stall in the daytime. If sudden, contraction necessarily involves lumeness; if very slow and gradual, lameness may not ensue, or may be deferred for a long time, but the first development of contraction is usually attended with lameness, more or less severe.

Symptoms._-Shuffling, or very slight lifting of the feet in motion, which occasions repeated stumbling; narrow: ing of the heels of one or both of the fore feet, especially of the inner heel; sometimes excessive liollowness and retraction of the sole, the foot being extended from the beel to the toe, but pinched (the crust on either side 
approximating) from side to side, the interstices on either side the frog sometimes almost obliterated. The animal always or generally stands with one of the fore feet stretched out in advance of the other, and if one foot only be affected, the foot so stretched out will be that which is so affected; or if both feet be affected, the projection of the feet will be alternated; sometimes the whole of the affected foot or feet may be observed to be, as it were, gathered up and pinched within an unnaturally small compass, and to offer very little surface to the ground; the retraction and indentation of the external form may occur at any part of the circumference between the coronet and crust, but is generally more apparent midway.

\section{Treatment.}

Give internally Siticea, Sulphur, or Thuja, and apply Oil of Tar and Neats-foot oil, in equal proportions, twice a day. Put on the patent shoe, as directed for "Navicular Lameness," at page 344.

\section{3.-Corns.}

A disease which occasions lameness, from the intense tenderness of the quarters (between which and the bars of the hoof it appears) precisely where the pressure is most severe. Unlike the excrescences of human beings, known by the same name, the corn of the horse consists rather in the softening than in the induration of the horn. The horn is occasionally observed to exhibit a reddish hue on the spot (of the corn), and it is always more subject to compression, but the animal appears to suffer acutely from the least pressure. 


\section{Treatment.}

Bathe with German Wash, three times a day. This will in many cases be all that is necessary, but if it fails to cure the corns, mix $1 \mathrm{oz}$. Oil of Tar with 2 drams Oil of Vitriol, and apply, three times a day.

\section{4.-Synovial Membranes.}

Nature has endued the bursæ and sheaths with a lining membrane, which secrets synovia, a fluid resembling oil, for the due lubrication of the parts.

It is not necessary here to enter into the physical structure of synovial membranes. It may be sufficient to say, that the outer coat of the membrane is thick, tough, and but slightly sensitive; whilst its inner lining is highly vascular and sensitive. From this lining is secreted the clear, bright, glistening, pale straw-colored sero-albuminous fluid, known as synovia or joint-oil.

When any cause, such as over-exertion, produces irritation in the part, the synovial membrane is excited by the irritation to throw out an increased secretion of oil. This increased supply must not be regarded as a evil in itself. On the contrary, it is useful in lessening the irritation, and is in fact a bountiful provision of nature for that purpose. Similarly, when a sprain occurs in the ligaments of a joint or in a tendon, an increased secretion of synovia is poured forth with the same object.

The liability to the occurrence of such causes is of course greatly dependent on the conformation of the animal. Upright shoulders, fetlocks, or hocks, and all 
other points of conformation which do not give due elasticity to the frame in action, are also liable to cause bursal enlargements.

Chronic inflammation of the joints, which is often found as a result of pneumonia, influenza, and sometimes of general debility, is another common cause.

Though overwork, sprain, faulty conformation, or chronic inflammation of the joints may be set down as the usual causes of bursal enlargements, yet they sometimes occur without any such violent exciting causes, and can then only be attributed either to a special irritability of the synovial membrane, on account of which it is excited to increased action on very slight provocation, or to weakness of the coats of the bloodvessels of the membrane, through which an undue effusion takes place.

\section{5.-Disease of the Horns.}

Disease of the horns is very common among cattle. The pith of the horn becomes festered, producing what is commonly called Hollow Horn - Festered Horn, or Horn Ail.

Symptoms. - The beast appears stupid and languid, refusing food; the eyes have a glassy appearance; the horn is very cold; the skin of the back and lymphatics becomes firmly contracted to the back and loins ; that becomes firmly settled to the back. Taking one hand hold of the end of the tail and the other above, you will often find it has become hollow, or soft, as if nothing but the skin held the brush of the tail to the bone. 


\section{Treatment.}

For internal application give Belladonna and Gelseminum, every hour, alternately, in 20 drop doses. For outside, wind the horns with a woolen cloth, tying it very tight at the base, then turn scalding water on to the horns. Take two tablepoonsfuls of vinegar, one teaspoonful each of pepper and salt. Mix and pour in the ear If a cure is not effected in a few days, repeat the dose. 


\section{CHAPTER XIX.}

\section{REARING OF SHEEP, SWINE AND POULTRY.}

\section{1.-The Various Breeds of Sheep, and their Productive Merits.}

The universal adoption and usefulness of the sheep as regards the necessities of mankind, cannot be better exemplified than by a glance at the universality of its location. Next to mankind, there is no living thing which is so universally domesticated, and so abundantly cultivated, as the sheep, or which has continued to engross the attention alike of the wandering hordes of semi-barbarous people, of every nationality, and of the most refined of civilized communities, as the sheep has done. The dog may, perhaps, be said to be as universally the companion, assistant, and favorite of man, but dogs are certainly not reared and nurtured in such numbers.

Europe, Asia, Africa, and America, all number their various breeds of sheep, each being more or less adapted to climate, circumstances, and locality. In Europe there are about twenty-five varieties, which are again subdivided into a multiplicity of local breeds. Those in 
the eastern and southern provinces of Russia (which also include some of the Asiatic breeds,) are famous for the delicate texture of the wool, which they yield abundantly. The Spanish sheep, which are short-wooled sheep, particularly those known by the distinctive name of Merinos, are unrivaled for the exquisite fineness, thickness, closeness, and abundance of their wool. The sheep of France include a great number of varieties, of which those of the northwest, towards the Flemish frontiers and the English coast, yield the largest quantity and the best quality of meat and wool, but they are by no means of a very superior class. The Saxon sheep, also short-wooled, which are Spanish sheep transplanted into Saxony, and which have served to improve the stock of the neighboring countries, especially to the extreme southeast of Germany, rank first in the world for the quality and abundance of their wool.

To these last may be added in succession, as to the comparative value of the wool (amongst the European breeds), those of Austria and its dependencies, of Wallachia and the adjacent provinces. The English breeds, such as they are actually, we shall presently have occasion to notice more at large, and shall therefore withhold any further remark in this place. Amongst the Asiatic breeds we notice more particularly the sheep of the Deccan, remarkable for good qualities of flesh, as well as for fineness of wool ; those of Persia generally, and of Kirman in particular, which yield a wool of peculiarly fine and delicate fibre; and those of Thibet, which possess no mean qualifications in respect of flesh, and the wool of which, like that of the Persian breeds, is of particularly delicate fibre. 
Amongst those of the British Colonies, exclusively of a few West Indian sheep, and exclusive of those of Canada, we have those of Australia, New South Wales, Van Diemen's Land, \&c., which, although they have not, until very recently, sent home to the mother country any wools of very high price, have been progressively improving as the colonies have been developed, and begin to constitute a very important feature in British commerce.

Besides the sheep of Canada, North America produces a few varieties of sheep, partly indigenous, and partly transplanted from England and elsewhere, whereby the aboriginal breed has been much improved.There are many districts in which the sheep yield a fair average quality and quantity both of flesh and wool. The South American sheep, which are chiefly attributable to Spain, have no doubt been equal to the merit of their extraction; but whereas they still retain some of the characteristics of the peculiarly fine wool of the migratory Spanish sheep, they have seriously fallen off from the aboriginal excellencies of the breed.

The African sheep are chiefly of the fat-tailed variety. There are, also, other varieties, partly due to European colonists, and partly to different localities. For the quality of flesh some natural historians praise the Barbary, Morocco, and Tunis sheep; some of which also are not without merits in respect of the fleece. The most interesting, perhaps, to an Englishman, are those of the Cape of Good Hope, which by various admixtures have been brought to considerable perfection.

Of British Breeds of Sireep.-Of the sheep which are generally bred in this country, we may divide the 
great majority into two classes, which distinguish them from those already described above as short-wooled sheep (under the heads of those of Spain and Saxony). British sheep generally are comprised under the heads of sheep with wool of medium length (middle-wooled shtep), and sheep with long wool (long-ucoled sheep), some of which are polled sheep, and some horned.

Amongst the former (the polled sheep) with medium wool, we may number the Herefordshire, Cheviot, Welsh, and South Down, and amongst the latter (the horned sheep), with wool of the same character, we may include the Norfolk, the Dorsetsbire, some of the Welsh breeds, the Heath sheep, \&c.

The Middle Wools.-This great class of sheep belong chiefly:

1. To the mountains of Wales and Scotland.

2. To the midland, eastern, southern, and southwestern counties of England.

Saving the exception of particular breeds of Lincoln and Leicester, or even Romney sheep, (which belong to the long-wooled variety) the middle wools may be considered the favorites of the British agriculturist. In such counties as Sussex, Hampshire, Somersetshire, Devonshire, Wilts, Hereford, Worcester, Cheshire, Kent, and Surrey, we find them universally prevailing. It is amongst the middle-wooled sheep that we have to class one of the most choice breeds of this country, viz., the South Downs, well known for the superior quality of their flesh, and found to yield from certain soils and with ample ranging scope, a by no means inferior quality of wool. These South Down sheep, which are now very generally bred throughout Great Britain, have one 
great merit which should strongly recommend them to the farmer, namely, that they are more healthy and hardy, and less subject to the ordinary diseases and casualties of sheep, than almost any other breer. Herefordshire is noted for one remarkable breed of sheep in particular, known by the name of Ryelands, which, although small, are unsurpassed for the quality of their wool. Amongst the Welsh breeds, that of Glamorganshire holds a high rank for merits at every point; there are also the Anglesey sheep, whose double merit of fattening easily and yielding very fine wool, has strongly recommended them to the attention of the breeder. Whilst in the north of England (in Cumberland), there is a breed known by the name of Hardwicks, which possesses great merit, both in respect of fattening properties, wool, and other features of some importance; whereas, the most eligible breed of all, that which belongs to the Cheviot hills, and has obtained the distinctive name of the locality, has successfully maintained its ascendency over the heath sheep or black faced breed, as it is called by some. The Cheviot sheep possess as much as, or more than, any other breed of English sheep, the three most essential qualities:

1. That of making flesh kindly, and consequently being one of the most profitable feeders.

2. That of being thoroughly hardy, and less affected by the accidents of sèson, \&c., than any other yet brought into the market.

3. Of yielding a particularly fine quality of wool.

Of the Scotch breeds, the Shetland sheep perhaps deserve the palm. Their wool is of a particularly suft texture, and remarkably white. 
The Long Wools.-In alluding to the different varieties of the long-wooled sheep, we will rather follow uniformly a direction northward, from the first district in which they are, as it were, indigenous, than a classification as to respective merits, more especially because these distinctions, in respect of a breed which does not vary in essential particulars of productiveness, are somewhat arbitrary, and because they are rather governed by the circumstances, more or less advantageous, under which they are reared, and in which they are sustained, than by natural superiority. To begin, therefore, in the extreme southeast, we first meet with a very valuable variety of the long-wooled sheep, in various portions of Kent. These have been peculiar to Romney Marsh from a very remote period; and although the wool is not fine, it is generally very sound and serviceable, most abundant, and very long. These sheep have also the merit of being exceedingly good and economical feed. ers ; they fatten very easily, and require but a very small share of nursing and attention. They have been improved by some admixture of the Leicester and Lincoln breeds. We again meet with the long. wooled sheep in the deep lands, and in the luxuriant valleys of Devon, Dorset and Somerset. In the rich lands of Worcestershire and Gloucestershire we meet with them once more, in the few remaining flocks of pure Cotswold blood, and in the mingled breeds descended from them. Their peculiar merit consists in the quality and abundance of their wool ; they are certainly inferior to the majority of the long-wools, and certainly to the Romney and Leicester breeds in respect of flesh-making. The full credit of the long-wools is centred in those of the midland coun- 
ties, and of the Leicester and Lincolnshire breeds, the former of which excels for the quality of flesh, and the kindliness of feeding, whilst the latter is the largest producer of wool of any of the English breeds, but is perhaps the most ungainly feeder of all the long-wools.These different merits are encouraged, to the depreciation of other qualities in each respectively, by the system of husbandry to which they are respectively subjected.

\section{2.-The Wool and its Peculiarities.}

In confining ourselves to the consideration of wool, in speaking of the natural covering of the sheep, it should not be understood, that it is exclusively provided with wool, but merely that the wool is the more valuable product. There are, more or less, admixtures of hair in the fleece, as there are, more or less, admixtures of wool in the coats of animals whose covering consists chiefly of hair. It is, however, the object of the breeder to free the covering of the sheep as much as possible from the presence of hair ; and this, as we shall presently show, is to be effected by the judicious treatment and constant attention of the breeder.

The wool is, in most cases, more or less improved or deteriorated by the abundance or scantiness of on oily alkaline substance, called the yolk, which evidently administers to the richness of the covering; and the yolk is always encouraged by the degree of shelter and warmth which the sheep enjoy, and by the quality of food upon which they are sustained. Where the breeding has been carefully and judiciously conducted, and 
proper attention has been paid to the progress of the animal, and to its protection from sudden changes of temperature, from severe inclemencies of weather, \&c. ; and when the situation and quality of the grazing grounds, and the quality of the artificial food, have been as well adapted as possible to the habits, requirements and origin of the sheep, the defects and irregularities of the wool will be gradually decreased. Wherefore, breeds of sheep which are indigenous to elevated localities, and to whom mountains perhaps are natural, should be fed upon upland soils, whilst the heavy long-wooled sheep, which has invariably been found in rich and irrigated plains, will thrive in low, marshy localities. Shelter from excess of heat, as well as of cold, is important, as the wool is as likely to be rendered coarse by excessive relaxation, and by the over-expansion of the pores, as to become wiry and harsh when exposed too inconsiderately to the utmost severity of a bleak winter. Nothing tends so much to render the wool irregular, the coarse being mingled with the fine, and the fibre being of various thicknesses, as exposure to great and sudden variations of temperature; whilst deficiency or unwholesomeness of food will quickly affect the color, elasticity and tenarity, which are so important, and the wool will lose its whiteness and with it other properties, whilst at the same time it will become patchy, and often also insufficiently thickened about the roots. The softness which we look for in wool will be affected by both contingencies, but by exposure in particular; whilst the intervention of a hairy admixture in the fleece, which should be so studiously avoided, will in general follow all irregularities in general treatment. 
The hair is distinguishable from the wool by being more regularly shed, and generally more than once in the course of a year, and that also more progressively, whereas the wool is cast in patches, if at all, and sometimes over the greater portion of the fleece at the same time. But it may also be stated, that the springiness of the fleece is comparative evidence of the absence of hair, which, from the absence of the systematic coil which conveys this elasticity, is devoid of the peculiar buoyancy which is conveyed to the touch, upon pressure, by pure wool, and which is due to the spiral curvature of the fibre.

With respect to the shedding of the wool, it is well known that the practice of shearing has obviated or forestalled this process.

\section{3.-The General Management, \&c., of Sheep.}

As with other domestic animals, the first great precaution which must be observed by the breeder is to select judiciously from his own stock, or to introduce new blood, in order that the productive qualities or excellencies of both male and female be as distinctively developed as possible; and in order that both be equally adapted to the climate, situation, and produce of the soil upon which the offspring is to be reared. In fact, it requires as much care, and as much attentive consideration of every peculiarity in the locality and in the animals, to breed successfully, as to rear successfully.Wherefore, at the earliest period at which the excellencies or defects of the lambs are observable, it were desirable to remove such as fall short of the characteristic 
good points of the flock in general, and to set them at once aside for fattening and for slaughter; so that they may not subsequently transmit their defects to a new generation, and thus gradually destroy the value of the breed. Besides these general regulations, another holds good of sheep, as well as of all domestic animals, and one, too, which it is the more important to impress upon the reader, that it is rendered imperative by the very care which the breeder takes to preserve the utmost integrity of his stock. It is ever desirable to select the ram as frequently as possible from other families and other loealities, with proper precaution, however, that the interloper have as closely as possible the characteristic habits, requirements, sc., of the floek to which he is introduced.

The Ewe: her Pregnancy, Yeaning, \&c- - In respect of the period of delivery it is important to consider the probable advancement of the season, in accordance also with the average condition of climate and veg. etation at such periods. If the locality be backward, cold, bleak, exposed, and elevated, the more desirable period of delivery will be later than in warm, forward, and sheltered valleys or plains. The most general period of lambing in this country extends from the first week in Mareh to the close of April; the March lambing being the more prevalent. The period should not be deferred until a season when there will, probably, be considerable heat, nor should the lambs be the least exposed at an earlier period, if the weather should happen to become unfavorable, and especially if there be a prevalence of cold, northerly spring winds. As, therefore, the period of pregnancy in sheep lasts a few days 
over five months, the month extending from the first week in October to the third week in November may be considered as the appropriate season for copulation, unless, indeed, if it be desired to obtain house lambs, when the ram should be introduced as early as the month of August. The copulation requires considerable care, lest, indeed, the ewes be not in such condition as to warrant a judicious breeder in propagating from them, and lest they be ill-served by the ram, who in such cases should be superseded. If the ram be inadequate to the service, the ewes will continue to follow him, whereas, if his duties be fulfilled, he will gradually have fewer and fewer followers. In the second autumn, both the ewe and the ram will be sufficiently developed for breeding; that is, if born in the month of March, and at an age varying from one year and seven to one year and eight months; but the ram in his first season is incompetent to serve more than about two score of ewes, whereas in his second and third season he is fully adequate to the service of a flock of from three to four score of ewes. And no sooner does it become apparent that the ewes are effectively breeding, than they should be tended with especial care. This condition is more immediately observable by the subsidence of the sexual evidences, and by their keeping aloof; and as this takes place they should be removed to a convenient and sheltered spot appropriated exclusively to them, and where they will be supplied with a sufficiency of generous food (in moderation). Good pasture and green winter food are advantageous, but should not be exclusively allowed; and care should be taken that they be not folded upon very wet and sodden soil, but that they be removed to dry 
places, in which also shelter must be afforded against the keen and cutting winds of uplands. Care should be taken that neither is the ewe gorged to excess with food, which is not uncommon where sheep have an unlimited access to some particular and favorite article, nor that she be allowed too scanty and impoverished a species of food, nor that there be access for the ram after pregnancy has once ensued, nor that she be harassed by the drivers, \&c., inasmuch as all these circumstances may induce a premature delivery ; when, though abortion be less frequent and less communicative amongst sheep than amongst cows, and though it be generally attended with little or no danger to the dam, there is no chance of rearing the lamb. There is, moreover, but very rarely an opportunity of arresting the course of a premature labor, because the ewe betrays but few premonitory symptoms until the process of ejecting the foetus has fairly set in. The indications of approaching abortion are akin to those already described under the head of the "Cow," \&c. If the abortion should actually occur, the ewe will require such appropriate treatment as will be found described at large in the article on "Abortion," in the directions for the treatment of disease.

\section{4.-Parturition.}

All risk of premature labor having been passed, we next arrive at the period of yeaning, or of parturition. As the time approaches at which some of the earlier ewes of a flock are expected to be delivered, the ewes should be confined within a comparatively small inclosure, and near at hand, that individual attention may be 
bestowed upon them. An inner fold should also be constructed with thatched hurdles, or some other material which may serve to afford shelter; and another portion of land, with abundance of keep (if possible), should also be parted off, wherein such of the ewes as yield twins should be conveyed. It is by no means disadvantageous, moreover, to confine all the ewes, which have not lambed during the night, within a smaller and sheltered inclosure or fold, so that they may be more readily watched by the person whose duty it is to superintend the lambing. This person will then continue to watch them night and day, always provided with the means of rendering mechanical assistance should it be required, and furnished with the medicaments which may be urgently and immediately called for, as well as with the means of marking the lambs, to provide against confusion, consequent upon the birth of many twins. In the meanwhile, also, the operation known by the name of clatting should be performed; this consists of clipping away the wool from about the thighs and udder, so as to render the milk as accessible as possible for the lambs.

But we do not wish to be understood to recommend direct interference during the progress of labor, unless this should become imperative from the duration of the labor and the exhaustion of the ewe, because there are comparatively few cases amongst sheep which demand any positive interference. But if the labor extend over a period of more than twenty-four hours, and the ewe should appear to sink under the continued suffering, or if the movements of the animal should indicate that the pains were evidently diminishing, and were inatequate to expel the foetus, medicinal or mechanical inter- 
ference would be called for. Or, again, if at any earlier period it should become apparent that mechanical obstructions to unassisted delivery existed, such a state of things would warrant an earlier interference.

It should, however, be held, as a general rule, that interference is objectionable, unless imperatively requisite. During the prevalence of cold, easterly winds, it is generally observed that the pains are less powerful than in a warm and moist state of the atmosphere, and consequently under the former circumstance the labor will naturally be of longer duration; and all these conditions should be duly considered. The same rules apply respecting the proper position of the foetus, and the mechanical means for the assistance of the dam, as those already described under the head of "Parturition," in respect of cows, saving that the mere introduction of the hand will suffice where operations are unnecessary, and that there is no necessity for the same application of power.

It is obvious that, to the breeder, the production of twins is an important source of profit; but twins are neither usual nor desirable at the first parturition. . It is from the second yeaning (or third year), to the fourth yeaning (or fifth year), that twins are generally yielded, and are most advantageous to the breeder.

At the first yeaning the ewe will not generally generate milk enough to sustain a double offspring.

There are frequent cases of three lambs being yielded at a birth; but in such cases it were desirable to leave one to be reared by hand, or by another ewe, because very few ewes are capable of nourishing three lambs at one time.

In the majority of cases, the ewe is fattened after her 
fourth parturition; and this is strictly in accordance with her capabilities, inasmuch as the absence of teeth incapacitates her from procuring sufficient sustenance for her own requirements and for those of her parasitic offspring. But when the teeth (as occasionally occurs), are not deficient, there is nothing to incapacitate the ewe from rearing two or even more additional lambs in sucsessive seasons.

\section{5.-The Early Treatment of Lambs.}

The first care should be to supply the place of the mother, in administering food to the lamb; and this is not unfrequently requisite, not only from the inability of the lamb to find the teat or to suck, but often from the timidity or temporary recklessness of the dam. The shepherd should be provided with milk, (ewe's milk if possible), in a bottle, whence he can feed the helpless or desolate lambs. It is almost needless to add, that this milk should be kept warm, by being carried in the breast as close to the body as possible. Even young ewes, in their first lambing, when they momentarily abandon their lambs, rarely abstain from seeking them out when the fulness of the udder begins to inconvenience them. Until, however, the lambs receive sufficient attention from the dam, in such cases it is incumbent on the shepherd to provide them with a sufficiency of warmth and food by artificial means. Where there are twins, it is desirable to remove the dam and her young to the fold appropriated especially to the twin lambs, as soon as possible. And in cases (of occasional occurrence with the first lambing), in which the dam absolutely forsakes 
her young, we may easily provide a foster-mother, if there be any ewe in the flock which has lost her lamb, by stripping the dead lamb of its skin, and folding the substitute therein for a time, until the ewe has got accustomed to her false young one, by means of the scent which originally deceived her. In cases in which a lamb is abandoned by the ewe, she should be folded apart with it, (the lamb being meanwhile fed by hand), until the dam becomes reconciled to it, and instinctively seeks to suckle it, to relieve the pain arising from distension of the udder. In cases of the first confusion, occurring amongst the twin lambs in the first days of their separate inclosure, constant attention will be necessary, as well to guard ewes which are deficient in strength, from being drained beyond their strength, as to provide for such lambs as may be rejected on all hands. With respect to the lambs, this attention will constantly be requisite, until they are able to provide themselves with a sufficient quantity of food, independent of the udder.Some will require to be reared by hand by means of the bottle, when they pine and fall away, and have evidently not sufficient strength to suckle of their own accord.

Lambs are rarely difficult of treatment in this manner. But if, after some time, and after the lamb has previously been suckled without artificial interference, we should notice an unaccountable falling off in condition, and an evident decline of health and strength, it will be desirable to examine the dam in respect of general health, and of the sufficiency and quality of her milk, and to provide against the ill consequences of any derangement, by proper treatment. If the deficiency be attributable to scantiness or poorness of keep, it will be necessary to 
obviate this mischief as gradually and carefully as possible, lest the too sudden transition should be followed by an inflammatory affection of the liver in the lamb, as is frequently the case. If the milk be productive of continued purging in the lamb, it will be evident that for some reason it disagrees, and the lamb should be artificially fed, or supplied by another ewe, if possible, for a time. As the lambs advance, so as to afford some idea of their future qualifications in the evidences of strength and kindliness, and in the quality of the fleece, we have an opportunity of making our selection of those which should be preserved for breeding, a reason why the habit of castrating or spaying very early is an error; because, with respect to the castration of the tup-lambs, of which very few will be preserved intact, it is quite as well to have the benefit of as much development as possible to assist us in making a judicious selection of the males, through which all the merits or defects of the breed are to be transmitted from one generation to another. And with respect to the spaying of the ewe lambs, from which we will have to make a large selection, the same advantage accrues from allowing the lamb as much growth and development as can be safely allower before the operation.

The operation of docking, which some breeders, or rather fanciers, repudiate, evidently tends to enhance the profitableness of the sheep, apart from the fact that it renders the lamb much less troublesome by removing a frequent cause of uncleanliness. The principal thing is to choose warm weather for the operation, but to afford shelter to the lambs from too severe exposure to the sun. The fact that warmth is important to the lamb after 
docking, should be a sufficient reason for not performing the operation too immediately after birth. There is not so much risk of inconvenience attending it in the threemonths-old lamb as in that of three days; and, as the season is then far advanced, the disadvantages attending the exposure to cold, \&c., are obviated.

The most important precaution to be observed in docking is, that the tail should be severed without cutting the bone, that is, in the partition between two of the vertebræ or links. The third joint is in general the point of separation. When the loss of blood is considerable, and continues for an undue length of time, it is sometimes found requisite to resort to mechanical means to arrest it, such as the binding round of the extremity of the tail-as it remains-tightly with stout thread for a short time.*

\section{6.-The Weaning of Lambs.}

As a general rule, we may wean the lamb at from fifteen to eighteen weeks old. But, inasmuch as the period of copulation is very important, the weaning must, in many respects, be accommodated to the probable time required by the dam to acquire her strength for breeding again; so that, on poor lands, and in ungenial climates, the period of weaning often ranges from ten to twelve weeks after birth; whereas, under very advantageous

* In the majority of cases, the use of tincture of Calendula is far preferable; and the following application should in general be adopted: To one part of tincture of Calendula add four parts of water, and bathe the bleeding extremity freely with the lotion occasionally, until the bleeding ceases; or even bind up the parts with linen rag, thoroughly saturated with this lotion. 
circumstances in respect of pasture and climate, the dam is not deteriorated in her breeding capacity if the lambs be not weaned until they have attained from twenty to twenty-five weeks of age. The lambs, at the period of weaning, should be removed to pasture more rich and productive than that on which they have been folded with the dams; but, if possible, there should be intermediate gradations in the quality of the food; for, whereas the lambs will require better independent feeding, it will also be advantageous to be very careful in affording them an increase in the abundance and richness of the pasture. The better method of parting the ewe and the lamb is as follows:

1st. To select a new pasture for the lambs, at a considerable distance from that upon which they were originally folded with the dams, consistent in abundance and quality with the above description.

$2 d$. To begin by folding the ewes and lambs together upon the newly-selected pasture which is intended to be appropriated to lambs only.

$3 d$. After the ewes and lambs have been together for several days upon the new pasture, to remove the former, either to the locality originally occupied by them, or to some other of an equally exhausted or impoverished description.

The separation will thus be rendered less apparent and painful, particularly if the ewes and lambs be so placed as to be beyond hearing of one another. So that upon farms of small extent (which, however, are ill-adapted to the breeding of large flocks,) it should be so contrived that, when separated, the ewes and lambs are at opposite extremities of the tract of land. 


\section{7.-Ewe's Milk; its Uses, Advantages and Disadvantages.}

* The custom of making use of ewe's milk, which is still adhered to in some parts of the Continent, requires some notice, for the very reason that it prevails; although, where the more profitable products of the sheep are properly considered, it will be found that the artificial extraction and adaptation of the milk reacts injuriously upon the fleece and flesh. In richness, the milk of the sheep is almost unequalled, but it possesses a peculiar oleaginous property which considerably detracts from the value and conservation of its products. The use of butter made from ewe's milk is thus almost precluded, from its tendency to become tainted almost immediately ; the cheese extracted from it is, however, much used, and yields a favorite commodity in some parts of Europe. Nor has the practice been totally abandoned in some of the mountainous districts of Great Britain and Ireland.

It is obvious, however, from what has been stated relative to the method of breeding and rearing of sheep, that the habit of milking ewes must be injurious.

1st. Because, if they be milked before the lambs are weaned, the lambs will necessarily suffer.

$2 d$. Because the interval between the weaning of the lamb, and the renewed appliance of the ewe to breeding purposes should, in order to render the ewe most profitable, be only sufficient to promote an adequate strength of constitution.

$3 d$. Because the artificial removal of this product will react to the deterioration of properties which are sources of far greater profit; namely, upon the wool and flesh. 
Moreover, it is impossible to obtain a firm and consistent butter from ewe's milk, owing to the oily nature of the cream. As in respect of all other products, however, it cannot escape the observation of the sagacious reader, that the relative advantages of adapting this or any other product to particular uses, must be regulated by the relative advantages which may be derived from them in particular localities. Wherefore if, in various parts of France, the custom of milking ewes for the manufacture of cheese be still adhered to, it is because that is found a better source of profit than either the fleece or flesh. Although, nevertheless, it is very questionable whether the last two might not be rendered yet more productive and profitable by the suspension of this source of produce.

\section{8.-The Uses of the Sheep's Skin.}

The uses of the sheep's and lamb's skin, with or without the fleece, are sufficiently known to excuse us from entering largely upon this topic. It should be observed, nevertheless, in respect of the comparative value of different skins, that whatever conduces to the fullest development of the general good qualities of the animal, in respect of flesh, and to the preservation of healthy action generally, will serve to enhance the soundness and value of the skin.

\section{9.-The Teeth, as Indications of Age in the Sheep.}

The complete set of teeth, in the sheep, consists of thirty-two, as follows: in the lower jaw, eight incisors, 
or cutting teeth, (two central and three on each side,) and twelve molars, or grinding teeth, (six on each side); in the upper jaw, twelve molars.

These teeth, however, like those of the horse, and of cattle, are of gradual development, and appear first of all as temporary or milk teeth (excepting two of the grinders) and are wholly perfected as a permanent set as early as the completion of the fifth year.

There is, however, frequently a variation of several months in the development of these teeth, according to the greater or less degree of strength, and to the quality and abundance of the food, and to a multitude of such qualifying conditions.

In the majority of instances in which the ewe is in good condition, and the lamb strong, the latter will be born with the two central cutting milk teeth; five grinders on each side, above (10), and the like number below (10) being present.

Like that of the ox, cow, \&c., the set of temporary incisors is completed within the first month, making them a complete set of milk teeth, twenty-eight in number.

But the temporary incisors cannot be said to be matured in less than from sixty to sixty-five weeks after birth, whereas, ordinarily, before the completion, we find the substitution of two central permanent incisors, and in the course of from thirty to thirty-five months we may observe another permanent incisor on each side of the two central teeth, making four altogether; each period of twelve months adding two more perfected permanent incisors to the jaw, until, at five years old, the sheep has completed its set of new cutting teeth, and has, in the meanwhile, also completed its set of permanent grinders 
by the gradual substitution of permanent for milk teeth, and by the protrusion of the sixth molar above and below on each side.

The wear, damage, and casting of these permanent teeth, after the fifth year, offers but a very imperfect clue to age, as circumstances will always hasten this state of things. But it should also be remarked that, saving for slaughter, the sheep becomes comparatively unprofitable after the fifth or sixth year, and at that age, the less time is lost in fattening the better, if it be anticipated to render the sheep of any value.

The construetion of the grinding teeth of the sheep is rather peculiar, which will explain for the casual observer the peculiar oblique method of mastication adopted by the animal. These teeth are furnished, on the grinding surface, with alternate ridges and indentations (running athwart the crown of the tooth, from the external to the internal edge), which indentations, \&c., are exactly matched by similar alternate irregularities on the crown of those of the other jaw, so that they correspond with and fit one another. Besides this, the crowns of the teeth above and below are matched, in respect of an oblique slope, those of the upper jaw being shorter at the outer than at the inner edge, and those of the lower jaw shorter at the inner than at the outer edge; the consequence of which (combined with the irregular surface above described) is that in the oblique action of the jaw all the food is secure of being thoroughly ground down. The age of the sheep can only be judged of by the degree of relative advancement of the teeth; there are no positive marks to be conveniently modified from time to time. 
416 BREEDING, REARING, \&C., OF YOUNG PIGS.

The difference between the temporary and permanent incisors is easily distinguishable in the size and in the sharpness, relatively to the general development of the frame, as evidence that they are too recent to have been much worn.

The first glance of the mouth would lead many persons to mistake the sheep at four years old for that at five, but upon closer examination it will be found that the two extreme incisors have become so much attenuated, and so overgrown by the larger permanent teeth, as to be almost totally concealed; whereas if they belonged to a sheep of five full years, the whole set of incisors would be equally developed.

\section{0.--Shearing.}

Shearing requires no particular notice, because all those who have tended sheep are sufficiently acquainted with the operation, and because nature herself, in the increased heat of the season and the natural shedding of the wool, has suffleiently pointed out the period of shearing, which varies only according to climate and circumstances.

\section{1.-Of the Breeding, Rearing, Weaning, \&c., of Young Pigs.}

The general merits of both the male and female from which we are desirous of propagating, for purposes of breeding, consist, as in all other domestic animals, of all 
the desirable qualities which are sought for in the offspring, and which are summed up briefly thus:

1. Kindly and economical feeders, and ready fatteners, yielding fine and delicate flesh.

2. Prolific parturitions.

3. Good nurses, and consequently successful in rearing their young.

In the boar we should look chiefly to construction, and to the consistency of such construction relatively to the breed of the sows, the locality, climate, and general circumstances. One boar, of desirable qualifications, may be deemed sufficient for the service of from eight to twelve sows. He should be between one year and four years old; and should be separately confined with the sows, one by one, and for a sufficient time only. If, however, the offspring of the first intercourse has been deficient in any important points, and that the characteristic merits of the sows be undoubted, this should be a sufficient reason for setting him aside, and for ceasing to breed from him.

The sows reserved for breeding, out of each litter, should be selected from amongst the most kindly of a prolific brood. There will be no incentive to retain young breeding sows from a scanty litter of less than eight pigs; nor should we any longer retain a sow for breeding purposes which has not farrowed at least eight pigs at her first parturition. No sow should be retained for breeding purposes which is not furnished with at least ten teats; all others should be spayed,* and reserved for fattening. A sow which has once been

* The general period of castration and spaying is about four wer ka after birth. 
guilty of rolling on her young, which has suffered a difficult labor, has proved a bad nurse, has failed to rear her young without any distinct, circumstantial cause, is in the habit of eating the after-birth, of devouring flesh of any kind, etc., etc., should at once be set aside for slaughter; she is utterly unfit for breeding from. A sow which has been delivered of a prolific litter at her first parturition (say of ten pigs), and which has proved an excellent nurse at that time, and has reared her young successfully, acquires considerable value. A sow of really good qualities as a breeder should rear at least two litters well every year, and may often furnish a third; but no sow should be retained for breeding more than three successive years. The average duration of pregnancy may be stated at nineteen weeks, although there is a variation of more than three weeks in this respect, between very young or very debilitated sows (which go a shorter time), and vigorous and matured sows (which go longer.) Every sow ought to be between ten and twelve months old, when first applied to breeding purposes, although, as a general rule, few sows are so old. If possible, time should be so measured that the two yearly litters may be farrowed as near the end of February and August (or a week or two earlier in both instances) as possible.

\section{2.-Preparatory Treatment, and Killing of Pigs.}

With respect to the preparatory treatment, the pig having been ascertained to be in good health, and perfect condition, little need be said, except the injunction, 
to withhold the food for an entire day previously, or to kill the first thing in the morning, and before any food has been administered. With respect to the killing of pigs, the inhumanity of the method of exposing the animal to the prolonged agonies of a lingering death is so obvious that any modification which could provide against this mischief would be worthy of notice. The most reasonable proposal is that of plunging the knife in such a manner into the throat as to separate the main artery. This, however, requires some experience; for very few of the ordinary bunglers who set themselves up as able managers of pigs would succeed in striking accurately, in many instances, in cases in which the animals were exceedingly fat.

\section{3.-Poultry.}

The importance and productiveness of poultry appear to be too much overlooked in this country. There are only a few districts in Great Britain where fowls of any kind are admitted to constitute a staple commodity for the market, and even then this consideration is confined to one or two varieties, viz., geese and turkeys generally; whereas, nowhere in the United Kingdom do we meet with the swarms of domestic poultry which hover about the homesteads of Picardy. The common domestic fowl, nevertheless, yields two very delicate, wholesome, and nutritious materials to the provision market - the flesh and the eggs, and possesses additional value, from the appropriation of the feathers to various branches of manufacture. 
The common Farmyard Fowl is divided, as it is bred in this country, into seven or eight varieties, and these again into a series of subordinate breeds, attributable to particular localities and the experiments of particular fanciers. Of these varieties, some are, more or less, purely foreign fowls, which have been introduced under the idea of improving the native stock. Such is the Spanish fowl. This breed is provided with black plumage, the bare parts being also black, and the comb and gills being large and very red. The flesh is much in request for the table on account of the whiteness and fineness of grain which characterize it, and the fowl is advantageously adapted for this purpose, owing to its size, in which respect it is second only to the Malay fowl and its combinations. Such also is the Malay fowl, the largest of the varieties known amongst the poultry in this country; the eggs are very large and abundant, and possessed of peculiarly nourishing properties - a remark which applies also to the flesh; but the latter does not possess the qualifications which constitute the fitness for the table; it is uncertain, never fine, and has always a darkish hue. The plumage is dark brown, with an occasional ray of yellow; the legs are long. As another distinct variety amongst the foreign fowls which have been introduced or naturalized in this country, we may name the Poland breed, whose merits, in the majority of respects, excel those of either of the two former, but which is not quite so large and much less gaunt. If anything, the flesh of this breed is more dainty than that of the best of our indigenous fowls. They fatten well and readily, and they yield an immense and never-failing profusion of eggs, which, however, perhaps as a 
natural consequence, are less in weight, thinner in shell, and sometimes considerably less charged with the peculiarly nutritive properties than those of the generality of our poultry. Like all other fowls, which are constantly laying eggs, the Poland breed are averse to batching them, and it is very rarely, in comparison, that they can be brought to devote themselves patiently and punctually to the offices of incubation. The better way, therefore, is not to depend upon them at all, but to place their eggs at once beneath a steady sitter. The characteristic features of this breed consist in their possessing more rotundity, obesity, and shortness of limb than either of the foregoing; in the glossy blackness of the plumage, relieved only by the mingled white and black, or even by a predominance of white in the tuft which overshadows the crown. In the place of a comb there is a circle of small, fleshy points.

Amongst the British breeds, we cannot but afford the first rank to the Dorking fowl. This breed is easily distinguished by a white plumage of a peculiar cream tinge, or sometimes of a sandy gray, with which, however, there is no admixture of feathers of any other color; by the presence of a fifth claw on each foot; by the shortness of the legs, in comparison to the size of the bird, which is next to that of the Spanish fowl ; and by the expansiveness and rotundity of the shape. The flesh is deep, sound, delicate, and fine-grained, and partakes of the creamy hue of the plumage.

The common Dunghill fowl cannot be numbered amongst specific breeds, inasmuch as it partakes in general of every variety, as is evinced in the endless variety of plumage, form, size, qualifications, etc. 
The Game fowl may rather be cited as the beau ideal of a fancier, than on account of any productive qualifications; for, although the flesh be unrivaled in whiteness, delicacy, and flavor, it is but a scanty layer, and still more incompetent of being reared successfully, and in numbers. The Game fowl will be readily distinguished by the ruddy, brilliant, and dazzling hue of its plumage, by the admirable symmetry of its shape, by the rapidity and activity of its movements, the fierceness and intolerance of its temper, and lastly, by the beautiful cleanness, slenderness, yet nervous and sinewy construction of the limbs.

Next in importance amongst English poultry to the Dorking breed, we may mention the Bolton gray breed, remarkable for the abundant supply of eggs, and for the whiteness of the flesh. It is appropriately named from its peculiar mottled-gray plumage, and distinguishable for the invariable snow-white fold in the plumage on either side of the neck. The interspersion of black spots throughout the feathers is more or less frequent, and the tail is generally marked with black rays.

The original breed known by the name of Shackbags, has been insensibly merged into new varieties, principally by the introduction of Malay blood, as also by crossing with other breeds, and is, we believe, no longer to be found in its integrity.

The pure Bantam breed, as remarkable for the yield of eggs, as for persevering incubation, besides the very superior quality of flesh, has earned for itself, however, a reputation rather with the fancier and rearer of game (for whose purpose it is unrivaled), than with the more mercenary peasant. 


\section{4.-The Breeding, Rearing, and General Man- agement of Poultry.*}

Selection of Breeding Cocks and Hens.-The breed from which these should be chosen is generally a mere matter of taste; but for purposes of profit, it is clear, that the selection should be made from such as possess most of the merits, and as few as possible of the defects, which are attributable to poultry. The cocks should, therefore, be possessed of high-mettled courage, and even of a savage disposition; the cock which has not the courage to offer a bold front, even against odds, is ever deficicnt as a breeder. The breeding cock should also be of such a construction as has been attributed to the characteristic formation of the most profitable breeds ; the body should be long and deep, the legs short, the breast broad and expansive, and rotundity should be selected rather than gauntness. Both the cocks and the hens should be healthy and vigorous, as evinced by the bright crimson hue of the comb and gills. Amongst the hens we should combine, in the aggregate, what is rarely combined in individual birds, by providing an adequate proportion of prolific layers and of patient and persevering incubators. We should avoid yellow-legged, or large-combed fowls, as a general rule, and we should pay particular attention to the indications of age. As breeders, cocks and hens alike, of about two years old, should be chosen; or even of three years old. Fowls which have not completed the second year are not advantageous breeders. But the advantage of selecting a first stock at the earliest age, when they are good and profitable breeders, consists in being provided with young substitutes, of adequate 
age, as the original stock declines; whereas, if we begin by selecting foyls in their prime (three years old), we shall not have young stock to replace them until the first stock has ceased to be eligible for breeding purposes. As the fowls grow old, we may observe the gradual fading of the bright redness of the comb, until it changes to something of a dirty yellow-red or brownred; we may also distinguish the increasing harshness, prominence, and size of the scales which cover the featherless parts of the legs; whilst the length of the talons, and the hardness, crustiness, and dull hue of the beak afford additional indices of age. The comparative number of hens; as respects the cock, should be as six to one. Some breeders prefer even a greater proportion of cocks, but we have in general found, that the excess was worse than the deficiency, and the proportion of eight to one will more frequently prove advantageous than that of four to one.

Selection of the Site, Soil, Conveniences, etc., for the Breeding and Rearing of Chickens. - The appropriateness of the situation should rule imperatively with those who seek for profit from the rearing of chickens. A dry, welldrained, and elevated situation is the best adapted for this purpose; and when the situation in which it is proposed to breed them is otherwise - as low, damp, illdrained, stagnant, or of a heavy, loamy soil - either some artificial provision should be made to provide against the ill effects of such a locality, or else the project should be abandoned. Fowls thrive best upon dry, sandy, or gravelly soils, and also upon elevations where chalk prevails. Districts which are adapted to the breeding of sheep are equally desirable for the breeding 
of chickens. In the absence of an appropriate soil and situation, a spot may be selected where there is a slope of southerly, southwesterly, or westerly aspect, whereupon an inclosure of strong and lofty fences may be erected, the bottom of the fences being sunk into the ground, to prevent the wandering of small chickens, and protected by the additional sinking of broken glass, and other hard and keen substances beneath the foundation, a precaution which may serve to exclude such vermin as would find means to burrow beneath, to get access to the fowls, whilst the upper ridge of the fences should also be well armed with tenter-hooks, to prevent access from that quarter. The soil should also be removed to the depth of two feet or more, and replaced, first by a considerable quantity of bricks and other broken building materials; and above these by layers of chalk and fine gravel, the upper surface being well hardened, and laid perfectly even to facilitate the proper cleansing of the inclosure. Heaps of fine coal ashes or sand should be placed in some corner of the inclosure, whereon the fowls may dust themselves. From the western extremity of the inclosure to the northward, and thence to the eastward, that is, on the side extending from the east by the north to the west, the sheds or roosts should be erected. Thus they will have a southerly aspect. They should be sufficiently lofty, thoroughly well covered and inclosed, and rendered proof against the access of vermin. The flooring or ground within should be such as that already described. From the floor upwards to the thwarts or perches (all of which should be on a level in the upper parts) should rise oblique (not perpendicular) ladders, consisting of a number of short rails projecting 
from the wall. The nests for purposes of laying should consist of a tier of parted shelves or boxes, not less than three feet from the ground, and accessible by means similar to those already described for the ascent to the perches. These boxes should be open in front only, provided with clean hay or straw, and guarded along the bottom of the opening in front by a strip of plank about four inches deep. The nests for incubating hens should be similarly constructed, with the addition of a sliding grated door, which may be closed at pleasure, to exclude the disturbance of the other fowls; but these nests, unlike those devoted to laying hens, should not be raised more than three or four inches from the ground, so that when hatched, the chickens may not be injured if they fall out.

If it can be so contrived that the side facing the inclosure can be constructed of moveable panels, this is a great advantage, because for the purposes of proper ventilation, the weather and temperature permitting, the whole or part of the frontage can be thrown open.

The same inclosure parted off for each respectively the whole of the appurtenances being similarly constructed . for the turkey, and the resting-places and nests differing only for ducks and geese (for which these conveniences should be on the ground-level) - is applicable to all domestic poultry. Because nothing will be easier than to provide an artificial pool in front of the shed in that compartment which is appropriated to the ducks and geese. If, however, there is no objection to a separate establishment, and a suitable sheet of water be at hand, it is not improper to notice, that it is yet preferable to erect all inclosures and buildings appropriated to the aquatic birds, on the brink of such a sheet of water. 
Care and General Treatment of the Poultry, Eggs, \&c., for Breeding.-If there be any arnongst the hens which are harassed and ill-treated by the cock, they should be removed; and the better way is to exchange them, providing also against the ill-usage of the substitute by its new companions.

Many fowls seem, from time so time, to have an imaginary inclination to sit, without any disposition to persevere in hatching their eggs; it may not be superfluous, therefore, to try the disposition of the hen for some days, with a number of false eggs, in order to ascertain whether or not she will abandon the nest. And if she then appears to persevere, it will be time enough to provide her with an adequate number of real and sound eggs for incubation; because, otherwise, if the hen should be fickle, all the eggs placed under her at first will be addled. As hens grow old, and do not moult until late in the season, or continue very long in the process of casting and changing their feathers, they become comparatively valueless, either for laying or sitting, inasmuch as they never lay except in the season of abundance, and then only a few eggs ; and as, moreover, they rarely possess sufficient breeding energy to hatch or rear their chickens.

The eggs which are reserved for breeding should be taken from the laying nests when quite fresh, as nearly uniform in size, etc., as possible, and exhibiting no flaws or irregularities in the shell. They should also be selected from amongst the full-sized eggs, lest they be those of the first season of pullets, which are generally smaller. They should not be kept longer than from twenty-one to twenty-five days, and, during that time, should be im- 
mersed in bran, and kept in a dark and cool place. Nor should we begin to reserve eggs for breeding until the spring is well advanced, lest there be none amongst the hens which are disposed to sit. Being provided with a stock of eggs for hatching, we will presuppose that some of the hens betray a disposition to sit, and have been proved, by the method above indicated, when we may select as many as can be well covered by the hen, carefully marking them with a pen or pencil, so as to detect any interloper (which may be deposited either by the sitting hen, or during her absence), and we may then place them in the nest which the sitting hen has already appropriated. There is a prejudice in favor of an odd number of eggs, eleven being about the average number. If, by accident, any of the eggs be broken in the nest, they should be removed, together with the adhering straw, etc., as soon as the fact is observed; and if the others have been rendered glutinous and sticky, they must be very carefully washed with water of a temperature of about $90^{\circ}$ to $110^{\circ}$ of Fahrenheit. .

The nests appropriated to the sitting hens should be constantly and thoroughly cleaned so long as they are unoccupied, and should be expressly cleaned and provided with fresh, short-cut straw or hay, before the eggs are placed under the hen. But, in all these operations, it is necessary to observe as much gentleness and expedition as possible, lest the sitting hen be driven away from the nest to which it is desired to confine her. A sufficient supply of fresh grain and clean water should then be placed within the reach of the hen, in troughs appropriated to this purpose only. These accessories should also be carefully renewed from time to time, care 
being taken to clean the vessels thoroughly on every such occasion, and to effect the removal with as little disturbance of the hen as possible. For the better effecting of these purposes, we may have the assistance of mechanical arrangements, whereby the grain and water troughs may be removed from the front of the nests without access to the interior of the shed, and without abruptly presenting ones self immediately in front of the nest.

Indeed, it might be added, that a separate compartment of the sheds should exclusively be devoted, if this can be effected, to the breeding fowls.

If the precaution of placing food, as above mentioned, within the immediate reach of the sitting fowls, be not adopted, they will often deprive themselves of food, in their obstinate perseverance in the duties of sitting.

Some hens have a far greater disposition to sit than others, and a few even never persevere until the completion of the period of incubation. But, whereas the eggs reserved for breeding should be placed only under good and patient sitters, these even should be so far discouraged from a constant disposition for incubation firstly, because they undergo such privations as consid. erably to weaken them; and secondly, because they do not devote a sufficient length of time to the rearing of the brood.

The length of the period of incubation is subject to slight variations, according to the various circumstances involved in the season, climate, temperature, state of the weather, persistence, or restlessness, or condition of the hen, \&c., \&c. Nor can we always calculate correctly from the period at which the eggs are assigned to the 
hen, which will sometimes feign to sit for some days before she actually does so.

Ducks and geese are subject to less variation in this respect than farm-yard fowls. The former almost invariably hatch in a month, and the latter in two days more. Hens sometimes sit as long as twenty-three days; but, under favorable circumstances, more commonly hatch their young on the twentieth. They have been known even to sit longer, or to hatch earlier.

In respect of pigeons, we have remarked an extraordinary difference, attributable to the different position of the dove-cotes. One instance has been most credibly reported to us, of four distinct nests of pigeons in the same dove-cote, immediately under a slate roof, having been hatched on the fifteenth day, or at least four days before the average period. The survivors of the pigeons thus hatched, amounting to five in number, it should be stated, were observed, one and all, to be more tame than the rest ; and, as it were, to seek the protection and intervention of their attendants. Two of them became entirely domesticated, and were oftener seen with the inmates of the house, by whom they were pampered, than with those of the dove-cote, by which they appeared to be harassed.*

The Hatching.-In proportion to the difficulty, danger, and inadequacy of mechanical interference in the liberation of the young of animals from their prenatal condi-

* This is a case illustrative of the deficient hardihood of animals, attributable to fotal or prenatal circumstances, and also of the extraordinary jealousy which animals in the state of nature, or in an approximate condition, entertain towards those which have become the objects of peculiar care and attention, or which are petted and pampered. 
tion, we remark the decrease of casualties attending such liberation naturally performed. Few animals which fall within the province of interference assigned to the reason of man, are subject to less difficulties at birth than fowls; whilst few, also, are so little susceptible of a practical and beneficial intervention.

With respect to animals whose parasitic existence (fotal) is completed before the completion of the animal organism ; that is, those which are twice born - once, in the emancipation of the egg, and again, in the liberation of the young animal from the egg - it is a strong argument against the artificial interference of man, that they are not even susceptible of the interference of their own kind.

The bird, of whatever kind, ow.es its final introduction into the animated world to the efforts of its own strength and energy alone. Sufficiently developed to dispense with its temporary inclosure, the young bird lustily ham. mers at the shell of the egg, to start into actual existence; and even a blow from the beak of the parent bird, guided and prompted by the natural instinct and affection for the fruits of its own labor, suffering, and privation, would endanger the life of the offspring.

The cases are, therefore, very rare, although they sometimes occur, when the interference of man, with the manifold appliances of his reason and ingenuity, are needed, or can avail to facilitate the completion of this second birth. In these rare cases, we may sometimes resort to the expedient of breaking the shell of the egg, but this must be done with great care, and very gently. The signal for such a course will be the suspension of further strong efforts on the part of the incarcerated 
chicken, after a struggle of many hours' duration. In the majority of cases (i.e., taking the average), the chicken does not effect its release until after twelve hours of labor, although instances are not wanting of the hatching being completed within periods varying from one to twelve hours. Whereas, on the other hand, these efforts have continued ineffectually for nearly two days. When the period of hatching is about due, according to our estimate, we should pay every attention to the nest.

A more frequent occurrence of the beneficial interference consists in aiding the removal of the membrane which incloses the chicken within the shell, and which will not unfrequently occur, owing to the inspissation of the size or white of the egg in which the chicken floats, by the heat applied during incubation, and the consequent adhesion of the membrane. The artificial removal of this species of skin, or film, can be affected with a little moderately warm water, which will serve to liquefy the size, and consequently to release the membrane on the one hand, and the chicken on the other.

As fast as the chickens are hatched they should be removed, deposited in a nest of wool, in a very warm situation, and there retained until the contents of every egg are extricated, when the hen must be cooped, and the chickens may be restored to her. No food should be given to the chickens (or, at least, not for twenty-four hours) until they are replaced. Nothing but sheer bruised grain, such as groats, should be offered to the chickens for some time, and the water with which they are furnished should be placed in such vessels as to prevent the chickens from falling into it, or becoming immersed. 
The coops of different broods should be as far removed from each other as possible; and after the first week, the hen may be liberated from the coop in fine weather, as soon as the dew is dried up, so that the hen and chickens may wander in search of their own food; but they should never be allowed (if it can be prevented) to be exposed in wet weather, or to run loose until the ground is dry. The chickens will continue with the hen until they are able to provide for themselves, unless, indeed, the hen abandons them; in which case, if they be seen to mope and pine, and fail, they must be carefully treated and reared by hand for some time longer. There is a peculiar wailing, painful, disconsolate chirp, whereby a young chicken signifies its sensation of helplessness.

\section{5. - The Feeding of Poultry for Market or Slaughter.}

The chief considerations in respect of feeding refer :

1. To the nature and quality of the food.

2. To the quantity.

3. To the method of feeding, and the contemporary general treatment.

1a. Without entering upon the consideration of the peculiar crotchets of particular breeders or fanciers in this respect, it will suffice to enumerate the articles which are most serviceable for this purpose; namely, entire grain, especially barley and wheat, and sometimes, also, oats, when they do not purge; malted or sprouting barley, grains, and other like products; meals of grain, especially of barley and wheat; animal substances, such 
as the ant's eggs ; or even pullets' eggs, boiled hard, and chopped up ; or, as recommended by some persons, unadulterated greasy animal substances generally. We may also name certain green vegetables and grasses, as conducive to the advantageous feeding of fowls. With respect to the quality, we have always found that there is greater economy as to actual cost, and that the results are infinitely more advantageous from using the best and heaviest grain, than from the use of refuse or tail corn, such as is commonly appropriated to the uses of the poultry.

$2 a$. With respect to the quantity of the food administered, that must be governed chiefly by the inclination of the fowls; generally speaking, the more the fowls contrive to consume the better.

$3 a$. The method of administering the food for fattening purposes resolves itself into the following proposition: 1st. The liberty to ramble in search of food, especially in farmyards, where, at the first thrashing, the fowls instinctively deriving the best of food from the refuse and waste of the barn, will fatten readily, and will yield the finest quality of flesh. 2d. Being confined, either to a section of the inclosure already described under the head of "General Management of Poultry," parted off as a feeding-ground; or being confined to the still narrower circuit of a coop; or even being deprived of light, to render the fowls more tranquil and indolent, and, consequently, less liable to waste a fraction of flesh, or fatty deposit; or again, with similar confinement, by what is termed cramming, that is, compelling the fowl to swallow a given quantity of barley or wheat meal, or of such other substance as may be 
deerned advisable; and lastly, by recourse to the opera tion of castration, with a subsequent similar course of feeding. The operation in question constitutes the variety of domestic fowls popularly called capons; a species of refinement on the ordinary treatment of poultry, to the employment of which three or four districts in England have undisputed claims.

If, however, young fattened chickens be in request, the best and true method of feeding consists in sustaining the condition which the chicken usually attains under the protection, and with the assistance of the parent fowl, by unremitting rich and generous food, never allowing the pullet intended for slaughter to degenerate into leanness, because such degeneracy, however temporary, will most materially deteriorate not only the quantity of the flesh, but the regularity with which it is interlarded, and, consequently, the juiciness and flavor.

\section{6.-Ducks and Geese.}

Ducks, like Guinea fowls, require watching as soon as the laying season sets in, that is, in February, lest they should steal away to conceal their eggs in some secret or inaccessible place.

There are very few varieties amongst ducks which appear to be indigenous, and these are either white, or have a great admixture of white or light-colored feathers. The Rhine Duck, for which we are indebted to the Continent, when pure, is distinguished by the prevalence of dark colors, and by the greater bulk of the bird.There are not nearly so many varieties of color and mark- 
ing, produced by hybridization amongst ducks, as amongst chickens; whereas, amongst geese again, we distinguish more varied admixtures of color. Muscovy ducks, which are occasionally found even about the homesteads of very rural localities, are but fancy fowls.

The Embden Geese seem to be held in estimation equal to that acquired by the white breed of ducks above named; yet of late years they have generally disappeared from the flocks. Geese, in their wild state, are the most uniform in color of any birds; they generally seem to correspond feather for feather. It is strange that, in their domesticated state, the uniformity has been replaced by the opposite extreme.

Ducks are miscellaneous and foul feeders. Offal of any kind, flesh, weeds, vegetables, and grain - the insects or small fish inhabiting the water which they frequent, \&c., \&c., are arnongst the articles which they consume.They seem to thrive and fatten near tan-yards, where they subsist almost exclusively upon the offal of animals; but their flesh becomes comparatively rank in flavor. Acorns are also found very advantageous for fattening ducks, and they seek for them assiduously in the vicinity of extensive plantations, or forests of oak. The best artificial food for fattening ducks seems to consist of mixed food, with the addition of a considerable share of crushed or bruised oats (especially), and pease. The meal of these kinds of grain may be considered the best form. Barley is to be avoided. The mingled refuse of the household cannot be better appropriated than to the feeding of ducks. Confinement is not preferable for fattening.

The staple food of the goose consists of the finer grass 
of permanent pastures, such as commons, waste lands, \&c., which, however, should be examined to see that it is free from poisonous herbs, such as hemlock, night. shade, \&c., which would destroy the goslings ; and situations should be selected where water is not wanting.Besides grass, if geese are intended for high condition, and to realize considerable weight, we should supply them twice a day with other vegetable substances, such as refuse cabbage-leaves, turnips, carrots, mangelwurzel, \&c., (carefully cut up), lucern, tares and the like. The range of stubbles, immediately after harvest, will fatten geese as well as, if not better, than artificial home feeding, especially if they have been kept in high condition by the means just described. We may begin to fatten the goslings in the course of the second month, when green food should be allowed only very sparingly. It may also be added that, where there are only a few geese, (or a few on which it is desired to bestow especial attention), we may very beneficially use crushed or bruised grain, (as described above in respect of ducks), mixed, if possible, with superabundant milk from the dairy; malt and fresh grains are highly advantageous as winter food for the geese, to forward them for breeding, and to obtain an early brood, so as to be able to rear a second in the course of the same year. Good pollard, sharps, \&c., also constitute very desirable fattening food, or food for goslings during the first three weeks.

The situation of the habitation appropriated to ducks has already been noticed, under the general head of Poultry.

Ducks are very abundant layers, and continue to lay until very late in the season. The period of incubation 
with ducks, extends on the average over a period of one month, more or less. If watched during the time that they are still laying, it will not be difficult to detect the hiding-places selected for nests, but for this purpose they must be watched at night or at the first dawn of day.The drooping of the belly, and the peculiar waddle of ducks heavy with eggs ready for deposition, betrays them, and if on the water the tail will be spread out, and close to the water, instead of being held up. Ducks lay at night, or very early in the morning, and rarely, if ever, after the day is advanced; and when they leave the nest, they invariably collect leaves and rubbish wherewith to conceal the eggs more effectually, a precaution which is also observed by the goose in rarer instances. According to its size and abundance of plumage, the duck can hatch twelve, thirteen, or fourteen eggs on an average, some even more. The proportionate number of drakes to ducks, should be as one to five.

The period of incubation with geese differs little from that of ducks, the average being a day or two over the month. The relative numbers of the male and female are the same as with ducks. The number of eggs covered varies from thirteen to seventeen.

The duck, during incubation, should not be disturbed, but should be left entirely to itself and perfectly secluded. Nor should the brood be meddled with until they are spontaneously brought out by the parent bird, when they should be cooped together upon smooth turf, or, in the case of the prevalence of very wet, cold, or changeable weather, they may better be cooped under cover within the hutches appropriated to them, the tails and the adjacent feathers being cut close. During this 
period whilst they are cooped, the soundest grain should be freely allowed to the old bird, as well as plenty of dry litter, constantly changed. In wet, unhealthy weather, good meal should be supplied to the brood and the old bird, the earliest food of the brood consisting of oatmeal. Ducklings should never be hatched under a hen where there is water at hand; and even when raised by one of their own kind they should be withheld from the water, (the mother being confined to the coop), for a period varying from a week to fifteen or eighteen days, according to the state of the weather.

A pan, containing a constant supply of fresh water, should be placed at hand, close to the coop. This is indispensable. The coops containing different broods should be far apart.

Geese, as the period of breeding approaches, should be carefully watched.

As early as the beginning of February, when the geese have been well fed on malt, grains, and the like, during the winter, we may notice them collecting straw and apparently busy in providing for a nest, when this should be supplied for them. A quiet place should be selected for this purpose, and straw carefully deposited.

During the period of incubation, when all the geese, which are sitting simultaneously, (if more than one), are collected in adjacent nests, the natural guardian, namely, the gander, which is ever at hand, will sufficiently provide for their protection and their wants. The early goslings will be hatched in the course of the month of March. The earliest food of the gosling should be much the same as that of the duckling. For the first fortnight the goose and her brood should be housed during the 
night, and during cold and wet weather, and should be folded in a small inclosure, set apart for this purpose, upon an adjacent grass-plat, as soon as the turf is dry, and the sun high above the horizon. At this early season the brood should be removed to the shelter appropriated to it for the night, at from three to five o'clock in the afternoon. When the goslings are from ten to fifteen days old they may be allowed the full scope of the pasture selected for them, with the parent bird, with the addition of the richer fresh food and grain above described; but, unless the season be far advanced, or if the nights be cold, they must still be housed at sunset.

\section{7.-Of Turkeys.}

The Rearing of the Chicks: During the completion of the process of hatching, the chicks must be removed and kept warm until the liberation of all is completed, when, for the first forty days, they will require to be kept under cover with the hen. They will then be fed with a thick mixture of oat or barley meal, and with chopped eggs, shreds of coarse boiled beef (unsalted) being soon added, the latter being strewed about to encourage the chicks to search for their food. Fresh-mown turf may also be afforded to them, but it must be thoroughly examined for the removal of noxious insects and vermin. As soon as they are allowed greater liberty, either by the cooping up of the hen in an open field, or by their being allowed to range abroad, a close watch must be kept over them to prevent their straying away, to which they are very prone, as well as to prevent the hen from straying from them, in her natural disposition to wander to a 
distance, without allowing the chicks an opportunity of keeping close to her. In the event of their being sickly, within a few days after hatching, a little crushed malt may be added to their food. Whilst they are kept in confinement it will be necessary to provide for the thorough cleanliness and dryness of the place appropriated to them. Grood, clean, but small shingle makes the best flooring.

Turkeys, it should be observed, are more difficult to rear than any of the other varieties of poultry domesticated in this country, and, therefore, all the precautions already prescribed for other fowls will apply yet more imperatively to these, besides those regulations which are specially adapted to their treatment.

After harvest, and later in the autumn, turkeys may very advantageously be driven over the stubbles and amongst oak plantations, whence they will derive much condition towards the fattening for the winter market.Where there are chestnut trees they may be allowed to range with still more advantage, but, in these excursions, they should be invariably attended and watched, lest they should stray away, as they are much in the habit of doing.

In the immediate vicinity of corn-fields, before harvest, they require no less attention; they scramble into the standing wheat, when they will destroy a great deal, and derive little good themselves from the mischief.

It is not uncommon, for the purpose of fattening for great weight, to castrate them in the same manner in which common fowls are converted into capons. At any rate, the mixed meals, derived from wheat, barley, and oats, beaten up into a thick pulp, with water, form the 
best artificial food which can be used to complete the fattening; such feeding, however, is only adapted to turkeys which are pounded for fattening. It will not be of so much service to birds which are allowed to range as the natural food which they will pick up of their own accord.

The proportion of the male to the females should be as six to one. The number of eggs upon which a turkeyhen may sit does not differ from that already mentioned of geese; and the period of incubation is of very regular duration, owing to the perseverance of the turkeyhen as a sitter-it may be estimated at thirty-two days.

The turkey-hen will frequently lay as many as from twenty to twenty-five eggs, so that in saving eggs for incubation, we should in general await the issue of her laying, evinced by the omission to lay at the recurring period, before we place the adequate number of eggs under her.

\section{8.-A Brief Notice of the Diseases Prevalent amongst Poultry.}

The diseases of poultry are generally attributable to one of two causes: Inclemency of climate and weather, or unwholesome food, with or without filth, etc. The great majority of the diseases (out of a comparatively small number) to which any classification has been assigned are of a catarrhal nature: such, for instance, as roup, chip, gapes; whereas others, such as vertigo, scouring, and costiveness (in some instances), pip, and vermin, are attributable to mistaken, excessive, or deficient feeding; and affections of the claws, skin, etc., are attributable to 
want of cleanliness, air, exercise, etc. With respect, therefore, to the treatment, as a general rule, we should indicate the modification of the cause; as, for instance, against the former class of diseases, warmth, protection from the weather, with healthy ventilation, dry housing, etc. And against the second class of complaints, a change of food, the withholding of miscellaneous food, the administration of well selected food at regular intervals, in small quantities, and carefully prepared; as also the thorough cleanliness of the roost, the removal of all noxious vapors and odors, and thorough ventilation.

In the treatment of Bone-wen, an affection which has been thought incurable, we have succeeded with Hepar s. and Silicea, five drops for each fowl every day, in the water apportioned to the fowls affected. We have also used Mercurius for Pip, and for Protrusion of the Ovary ; Bryonia, Allium, and Arsenicum in Roup; Opium and Sulphur, in Gapes and Constipation; Ferrum a. (a popular remedy), Secale and Sulphur, against Scouring, with great success. Agaricus m., and Calcarea, during Moulting, also with considerable success. Colchicum and Sulphur for Chip; China and Staphisagria for the spontaneous generation of Vermin; as also Tobacco (the last externally), followed by Pulsatilla (internally), in the same case; Mercurius, China, and Silicea, against Core; Cannabis against general and unaccountable Emaciation.

The Bone-wen is an enlargement or excrescence, forming about the bones of the joints, similarly to that to which we have referred in respect of the horse, under the head of "Splint."

Pip is a complaint characterized by the formation of a white scale at the extremity of the tongue. The fowl 
begins by moping, and keeping apart, refuses to eat, and is dilatory and irregular in its motions. Costiveness is most frequently an attendant symptom, and we sometimes find considerable heat, especially about the belly, close to the thighs.

Roup is a term which includes a variety of catarrhal affections, arising out of exposure to changes of temperature, wet, etc., etc. One of the characterizing features of the disease, in an advanced stage, consists of discharge from the nostrils; the eyes are also occasionally involved, being swollen and inflamed.

Chip or chipping, so called from the peculiar cry emitted by the bird, is another disease, which partakes of the general character of roup. It is confined to young chickens, and is very fatal if not taken in time; but Colchicum has been administered with admirable results in the earlier stages. It arises from wet weather, the light down of young birds becoming easily saturated and long in drying. The feathers droop, and lose their gloss; the chicken is extremely tender of being touched; there is considerable fever, and external heat is remarkably developed, although the chicken sits trembling violently and continually.

Scouring and costiveness need no particular description.

Core consists of the formation of a species of deposit, or excrescence, either in the gullet or alvine passage. The core varies in color, sometimes being brownish-yel. low, sometimes darker, and sometimes of the color of ochre intermingled with red.

Gapes, or the yawning disease, so called from the prevalent characteristic symptom, is another modification of roup, and its subordinate catarrhal affections. 


\section{SMALLPOX IN MAN.}

This disease is, by medical practitioners of the present day, divided into two varieties: the distinct, when the pustules on the face are clearly defined, and do not run into each other,- and the confluent, when they coalesce and form one continuous whole.

When the symptoms are less severe than those properly characteristic of the disease, and the eruption on the face slight, it is called the modified small-pox. We generally find this description in such persons as have been properly vaccinated.

Symptoms.-The disease is frequently very sudden in its attacks, commencing with chilliness and shivering, followed by a high fever, headache, severe pains in the small of the back and loins, languor, weariness and faintness; the voice of the patient is also changed, and he complains of oppression of the chest, and acute pain in the pit of the stomach, increased by pressure. The eruption makes its appearance on the head at the close of the second day, during the third day on the face and neck, and afterwards spreads over the whole body.

The eruption first displays itself in the form of small, hard-pointed, red elevations, which, in about three days, present a bladder-like appearance, surrounded by an inflamed circular margin, and become depressed in the centre as they enlarge. About the sixth or eighth day, the watery secretion in the pustule becomes converter into matter, and the depression in the centre disappears. 
When the pustules are very numerous on the face, it generally becomes much swollen, and the eyelids are frequently closed up. On the first day, a small lump, like a millet seed, may be felt in each of the elevations above noticed, distinguishing this eruption from all others.

In Confluent Smallpox, all the precursory symptoms are more severe, the fever runs high, and frequently continues so throughout the course of the disease; the pain in the pit of the stomach and difficulty of breathing are more complained of, and in children the eruption is frequently preceded by convulsions and delirium.

\section{Treatment.}

On first appearance of fever in cases of Smallpox, take Aconite and Belladonna, alternately. When eruptions appear, take Bryonia. If nervous, take Chamomilla. If troubled with nausea or vomiting, take Pulsatilla. Rhus Tox is a good remedy when rheumatic pains are felt in the back and extremities. Stramonium is particularly serviceable in forwarding eruptions.

Prepare the above remedies by mixing five drops of solution in half a glassful of water. Give one teaspoonful every hour, except in the case of Aconite and Belladonna, which should be given alternate hours.

As the pustules become filled, open them with a sharp instrument, and their discharge will prevent all disfiguration, and relieve the disease.

Fresh air is our best auxiliary, the emanations from the patient in this complaint being of a nature to react upon the organism, and warmth being calculated to increase its activity. Great cleanliness must also be observed, and the linen frequently changed. 
APPENDIX. 


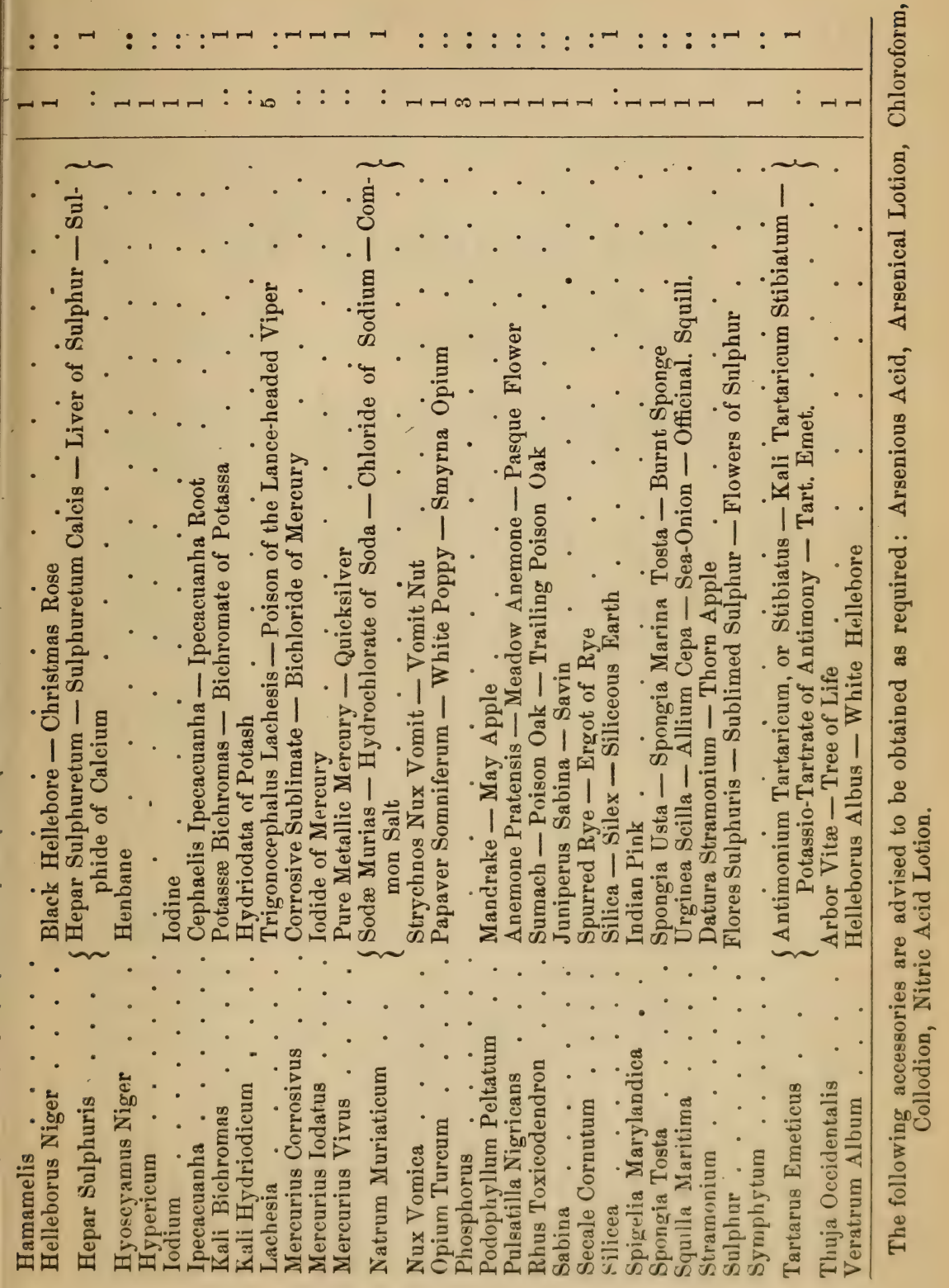




\section{EXTERNAL APPLICATIONS.}

This class of remedies includes certain specified drugs which are applied to the external surface of the body, in local affections, or injuries, for three distinet purposes firstly, to assist the operation of the given drug when it is administered internally at the same time; secondly, to obtain a disinfectant, or antiseptic action in cases of unhealthy ulceration, or sloughing; and thirdly, to destroy vermin.

The following is a list of these applications, and their principal uses:

\section{Arnica Lotion.}

Mix 1 fluid ounce of Tincture of Arnica with 15 of water.

Used in all kinds of injuries caused by mechanical violence, such as falls, blows, etc.; in fractures, dislocations, bruises ; to wounds after operations; to pricks of the fiet, corns, etc. In the last cases, a piece of tow soaked in the strung tincture should be applied; and a bran poultice may be medicated with the same, or with some of the lotion.

\section{Calendula Lotion.}

Mix 1 ounce of Tincture of Calendula with 15 of water.

Used in cuts, lacerations, flesh-wounds, etc. When the wound is irritable and inflamed, and a poultice is required, add to it two or three wine-glassfuls of the lotion. 


\section{Rhus Lotion.}

Mix 1 ounce of Tincture of Rhus with 15 of water.

To be well rubbed into the seat of sprains of ligaments, or tendons, and in some forms of local rheumatism.

\section{Kali Bichromicum Lotion.}

Dissolve 1 dram of the salt in 16 fluid ounces of water.

To be rubbed over farcy-buds, the enlargements of weed and cellulititis; also very efficient in capped hocks, after Arnica Lotion.

\section{Ruta Lotion.}

Mix 1 ounce of Tincture of Ruta with 15 of water.

Used in some cases of distension of joints, or bursal sacs, resulting from sprains. To be rubbed in.

\section{Arsenical Lotion:*}

Boil 4 grains of Arsenious Acid in a pint of distilled water.

Used in some forms of cracked heels, grease, and mange.

\section{Mercurius Corrosivus Lotion.*}

Dissolve 1 dram of Corrosive Sublimate in 16 ounces of hot water.

Used as an injection in fistulas, quittor, etc. ; in the reduction of splints, bone spavins, ring-bones; in thickening of the sheaths of tendons ; and in some parasitic diseases of the skin. When rubbed in, the skin gets tender and scurfy in a few days; it should then be discontinued for a day or two, and the part rubbed with oil, and washed well with soap and water; then resumed.

* To prevent accidents with these two applications, the bottles containing them should be labelled "POISON," and, when not used, locked up. 


\section{Iodine Lotion.}

Tincture of lodine 1 to 2 fluid drams; water 16 ounces.

Used in vaginal discharges.

\section{Kali Chloricum Lotion.}

Kali Chlor. 2 drams; Glycerine 8 fluid ounces; water 8 ounces.

Used in foot diseases of cows, grease, etc.

\section{Belladonna Lotion.}

Extract of Belladonna 2 grains; water 1 ounce.

Used as a local application in periodic oplthalmia.

$$
\text { Hydrastis Injection. }
$$

Tincture of Hydrastis 1 ounce; water 16 ounces.

Used in nasal discharges ; vaginal discharges.

$$
\text { Sulphur Ointment. }
$$

Sulphur 1 ounce; lard 6 ounces.

Used in parasitic skin diseases, and pimply eruptions with itching.

$$
\text { Benzine (Undiluted.) }
$$

Used in parasitic mange - page 265 .

Sulphurous Acid, and Lotion.

Used pure in malignant putrid sores, and unhealthy ulcerations; and diluted with equal parts of water, or of Glycerine, in certain skin diseases.

$$
\text { Hydrastis Inhalation. }
$$

1 dram of the powder; water 1 quart.

Used in nasal discharges. 


\section{Carbolic Acid.}

Mix 1 ounce with 20 ounces of water.

Used in all kinds of putrid sores, as foot rot, glanders and farcy, foul discharges from the head. Also for proud flesh, skin diseases, parasites, and as a disinfectant in malignant and infectious diseases.

\section{Baptisia Inhalation.}

Tincture of Bxptisia 2 fluid drams; water 1 quart.

Applied by means of a specially constructed apparatus, or by pouring some of the liquid on a hot brick held under the nose. In abscess of the lungs - page 181.

\section{Tobacco Fumigation.}

The Tobacco to be burnt on a hot shovel, placed under the animal's nose.

To kill the parasites in the nose, and in bronchitis from worrns.

Sulphur Fumigation.

Sprinkle some Flowers of Sulphur on hot cinders held on a shovel, placed under the nose of the animal.

To kill parasites in the nose, and used in bronchitis due to worms.

$$
\text { Thuja (Undiluted Tincture). }
$$

Used for warts, applied externally, page 272 - and mallenders, page 273.

Powdered Slaked Lime.

Used in open joints - page 53.

\section{Tincture of Urtica (Urens) Dioica.}

This remedy has been found specific against the stings of insects. It should be selected preferably to Arnica, when the stings have produced considerable swelling and there is burning heat and excessive tenderness of the surface to the touch, so much so that the ani- 
mal shrinks from examination, and that when touched the skin quivers.

The Urtica Urens will be found useful in all burns and scalds, when the integuments and subjacent tissues are not destroyed, and when the injury is confined to the skin.

When used for stings, the pure Tincture of Urtica may be touched to the affected part once in half an hour, or once an hour, till the swelling begins to go down.

When applied to burns, this remedy will more quickly relieve the inflammation if mixed with warm water, and applied quite warm, once an hour. For burns, the lotion should consist of six parts of pure soft water to one of the tincture. At first, in severe cases, the lotion should be used afresh every quarter of an hour, by means of a linen rag bound about the parts, and resaturated at such intervals; but, when the severest effects of the injury bave been overcome, it will be sufficient to remoisten the rag at intervals of three hours during the first day, and to bathe the parts with the lotion twice a day afterwards. 


\section{DIRECTIONS}

\section{For Compounding Medicines}

PRESCRIBED IN THIS WORK.

When purchased of a Homeopathic Pharmacy, add one dram of Tincture to ten drams of Alcohol.

When purchased of ordinary druggists, add one dram of Tincture to six drams of Alcohol.

One grain of any of the medicines in powder, is equal to one drop of the liquid. For convenience in using, dissolve one dram of the powder in one ounce of water, then add two ounces of alcohol, and the solution will be of the right strength for twenty-drop doses. 


\section{RECIPES.}

We give here several recipes for the cure of bony enlargements, which have all been used with success in different cases :

3 oz. Euphorbia ; 1 oz. Cantharides ; $2 \frac{1}{2}$ drams Iodine Crystals; 3 drams Red Precipitate; 2 drams Bichloride Mercury; 1 oz. Mercurial Ointment ; $\frac{1}{2}$ oz. Venice Turpentine; $\frac{1}{2}$ lb. White Pine Turpentine; $\frac{3}{4} \mathrm{lb}$. Lard. Melt the Lard and Pitch together; then put in one ingredient at a time, as the recipe reads. Apply what will well cover the enlargement; rub with naked hand, and let it remain two days, and then grease. The third day wash, the fourth day apply as before; and so on until the enlargement is gone. When applied to the shoulder, use 25 per cent. more lard.

2 oz. Cantharides; 4 oz. Mercurial Ointment; 3 oz Tincture of Iodine; $4 \mathrm{oz}$. Turpentine; 3 drams Corrosive Sublimate. Mix all well with 2 lbs. of Lard. Cut off the hair from the part affected, grease the lump with the ointment, and rub it on with the naked hand. In two days grease the part with lard, and in four days wash it with soap and water, then apply the ointment again. So repeat until a cure is effected. For Wind- 
galls, Bog Spavin, or Curb, apply the ointment every six days.

1 oz. Cantharides; $2 \frac{1}{2}$ oz. of Muriated Acid; $1 \mathrm{oz}$. Laudanum; $1 \frac{1}{2}$ oz. Spirits of Turpentine; $\frac{1}{4}$ oz. Sul Zinc; 1 oz. Tincture Iodine; $\frac{1}{2}$ oz. Origanum; 1 oz. Castile Soap; $\frac{1}{2}$ oz. Camphor Gum; 1 oz. Rosin ; $\frac{1}{2}$ oz. Venice Turpentine; $\frac{1}{4} \mathrm{oz}$. Corrosive Sublimate; $1 \mathrm{lb}$. of Lard. Melt the Lard, then add Muriated Acid. When melted, add the balance, and stir until cold.

1 oz. Spanish Flies ; 2 oz. Benzine. Apply for three dayș, then grease with Lard.

$\frac{1}{2}$ gill Spirits Turpentine; $\frac{1}{2}$ oz. Corrosive Sublimate. This will sometimes cure when nothing else will reduce the enlargement, but care must be taken not to use it too freely.

For CURB or Splints.

2 oz. Oil Turpentine ; 2 oz. Camphor; 2 oz. Origanum ; 1 oz. Spirits Ammonia; 1 oz. Olive Oil. Shake it well, and apply with a sponge, without rubbing, three or four times a day, until it scabs.

For Thoroughpin, Bog Spavin and Windgalls.

1 pt. good Soft Soap; $\frac{1}{4}$ lb. Brown Sugar; 2 oz. Spirits Turpentine; 1 tablespoonful of salt.

It is good also to apply to bone spavin, when it has been blistered. Apply twice a day, and rub in well with the hand.

\section{Chloroform Liniment.}

Mix Chloroform and Cod Liver Oil. Good to relieve suffering in case of burns, etc. 


\section{Black Liniment.}

$\frac{1}{2}$ pt. Linseed Oil ; 3 oz. tincture of Iodine ; 4 oz. Turpentine; 1 oz. Oil of Origanum. Shake it well before using. This liniment is good on any swelling. .Wash the part clean with soap and water before applying, and rub the liniment well in with the hand.

\section{Muscle Liniment.}

1 oz. Oil Origanum; $\frac{1}{2}$ pt. Alcohol ; $\frac{1}{2}$ oz. Oil Cedar; $\frac{1}{2}$ oz. Oil Cloves; $\frac{1}{2}$ oz. Turpentine; 8 oz. Olive Oil. Shake it well. This is used for almost all complaints of the muscles.

\section{OPODELDOC.}

$\frac{1}{2}$ gal. Alcohol; 2 lbs. Castile Soap; 4 oz. Gum Camphor; 2 oz. Oil of Ambre.

Pour the Alcohol into a pot, and set it in a kettle of hot water. Shave up the soap, and put it into the Alcohol with the other ingredients. Keep hot until all is dissolved.

This is the old original Opodeldoc.

\section{German Wash.}

1 quart of good, strong Cider Vinegar, 2 oz. pulverized Blue Vitriol, 3.oz. Alum, 3 tablespoonfuls pulverized Loaf Sugar, 6 tablespoonfuls Honey. Simmer together in an earthen or pewter vessel. For human flesh, use with the same amount of vinegar one-quarter of the other ingredients.

This will be found an excellent remedy for all kinds of sores, whether simple cuts or bruises, or unhealthy ulcers, or fever sores. 
For Sprains, Bruises and Lameness.

Two ounces of each of the following ingredients : Oil of Spike, Origanum, Hemlock, Wormwood, Spirits Ammonia, Camphor Gum, Spirits Turpentine; 4 oz. Sweet oil; 1 quart Proof Spirits, 95 per cent. Mix well together and bottle tight.

The above liniment cannot be equaled, and will be very useful to any one keeping valuable horses. Omit the Turpentine, and you have the best liniment ever made for human ails, such as rheumatism or sprains. Whenever an outward application is required, try it and prove its virtue.

\section{Rheumatic Liniment.}

$\frac{1}{2}$ pt. of Alcohol; $\frac{1}{2}$ oz. of Origanum; $\frac{1}{2}$ oz. of Cayenne Pepper; $\frac{1}{2}$ oz. of Gum Myrrh; 1 teaspoonful of Lobelia. Let it stand all day, then bathe the part affected.

\section{SOAP Liniment.}

1 oz. Origanum; 1 oz. Castile Soap; 1 pint Alcohol Used for swellings, etc.

Ointment for Cuts and BRuises.

4 lbs. Mutton Tallow; $\frac{1}{2}$ lb. Beeswax; $\frac{1}{2}$ lb. Rosin; 3 oz. Turpentine. Melt over a slow fire, and when nearly cold, add the Turpentine. Good for cuts and bruises.

\section{Butter Ointment.}

2 lbs. of fresh Butter; $\frac{1}{2}$ oz. Tincture of Iodine; $2 \mathrm{oz}$. Oil Origanum. Mix well for fifteen minutes, and it is ready for use. This salve is good for any inflammation on man or beast, - rheumatism, sprains, burns, swell$20^{*}$ 
ings, bruises, chapped hands and lips, or black eyes. Apply it every night, and rub well in with your hand. For human flesh, put it on warm flannel.

\section{Blue Ointment.}

$4 \mathrm{oz}$. Ointment of Rosin; $\frac{1}{2}$ oz. of finely ground Verdigris; 2 oz. of Turpentine; 2 lbs. Mutton Tallow; $\frac{1}{2}$ oz. Oil Origanum; $\frac{1}{2} \mathrm{oz}$. Tincture Iodine. Mix all well, and this is one of the best medicines that can be made for scratches, hoof-evil, and cuts. It is good to apply on fistula, after the pipes have been taken out.

\section{Hoof Ointment.}

4 oz. Rosin; 6 oz. Beeswax; 2 lbs. Lard. Melt together ; pour it into a pot, and add $3 \mathrm{oz}$. of Turpentine, $2 \mathrm{oz}$. of finely powdered Verdigris, $1 \mathrm{lb}$. Tallow. Stir all until it gets cool. This is one of the best medicines for the hoof ever used. It is good for cork or bruises of the feet.

\section{Treatment of Hoof Bound, or Tender Feet.}

If the part is contracted, have the foot spread at the heels, but never rasped above the nail holes. Use either the hoof ointment or hoof liquid. Apply all around the top of the hoof down one inch, every third day. For split hoof apply every day, first cleansing the cut or crack, and putting a stiff shoe on the foot. Never cut nor burn it. Oil of Tar and Fresh Butter is also a very good remedy.

For Fouls in Cows Feet.

$4 \mathrm{oz}$. Oil of Tar ; $1 \mathrm{oz}$. Oil of Vitriol. Apply twice a day to the infected part. 
Drops to Make Old Horses Young.

1 oz. Tincture Assafoetida; 1 oz. Tincture of Cantharides ; 1 oz. Oil Cloves ; 1 oz. Oil Cinnamon ; 2 oz. Antimony; $1 \mathrm{oz}$. Fenugreek; $\frac{1}{2}$ gal. fourth proof Brandy. Let it stand ten or twelve days, and give ten drops in a pail of water, or 1 gallon.

\section{For Cleansing the Blood.?}

$\frac{1}{4} \mathrm{lb}$. of Blood Root; $\frac{1}{4} \mathrm{lb}$. of Licorice Root; $\frac{1}{4} \mathrm{lb}$. of Fenugreek; $\frac{1}{2} \mathrm{lb}$. Linseed Meal. Give small tablespoonful every twenty-four hours.

For Cough, and Diseased Liver or Lungs.

1 oz. Ginseng, bruised; 1 oz. Gentian, pulverized; 1 oz. Bitter Orange Peel; 1 oz. Chamomile Flowers; 1 oz. Horehound; $\frac{1}{2}$ dram Aloes; 1 oz. Spikenard; $1 \mathrm{oz}$. Dandelion. Add the above to $\frac{1}{2}$ gal. Water and $\frac{1}{2}$ gal. good Whisky, let it stand twenty-four hours, and take a tablespoonful three times a day.

This is the best medicine for consumptive people I know of, and is published in this book by request of many who have proved its merits.

Antidote for Poison - Man or Beast.

White of an Egg and Honey, beat together. Take tablespoonful every half hour, until relieved. 


\section{GLOSSARY :}

\section{OR EXPLANATION OF SOME TECHNICAL WORDS OCCURRING IN THIS BOOK.}

Abscess - A collection of matter.

Absorbents - The absorbent vessels pervade all parts of the body, and take up and convey matter, not altogether useless, to the thoracic duct.

Anthrax - Carbuncle.

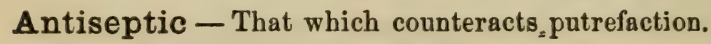

Arthritic - Pertaining to joints.

Articulation - A joint.

Ascites - A collection of fluid in the belly.

Astringent - Applied to plants, etc., that contract animal fibre. ${ }^{* 1}$ Bronchial Tubes - The branches of the air-passages in the lungs. Bronchocele - Enlargement of the thymus gland in the neck.

Cachexia - Bad habits of body, in which the fluids and solids are vitiated.

Caries - Ulceration of bone.

Calculus - A stone,

Calculi-Stones.

Catarrh - A cold.

Characterized - Marked by particular symptoms.

Chronic - Long-standing, as opposed to acute.

Coma - Deep drowsiness.

Comatose - The state of coma. 
Concussion - The disturbance caused by shaking.

Conjunctival $\mathbf{M}$ embrane - The membrane lining the eyeball and eyelids.

Congenital - Applied to diseases, or malformations existing at birth.

Conjunctivitis - Inflammation of the mucous membrane lining the front of the eyeball and the eyelids.

Constipation - Bound bowels.

Contagious - "Catching."

Contagion - The transmission of disease by contact with the morbid matter.

Convalescent - Recovered.

Convulsion - Violent shaking of the body, such as occurs in fits.

Cornea - The clear portion of the eyeball in front.

Coryza - Cold in the head - nasal flux.

Dentition - The process of teething.

Diagnosis - The act of "seeing through" diseases, and so distinguishing one from another.

Diaphragm - The midriff. The muscle which separates the cavity of the chest from that of the belly.

Digestive System - The organs concerned in digesting food.

Dyspnœa - Difficulty of breathing.

Ecchymosed - Stained with extravasation of blood under the skin, or mucous membrane

Efflorescence - A condition of the skin in which there is general redness.

Emaciation - Wasting.

Emphysema - Applied to the state of the lungs when the air-cells are ruptured, or dilated.

Engorgement - The blood being present in too large quantity in the lungs.

Epidermis - The outermost layer of the skin.

Epileptiform - A convulsive attack like that of epilepsy.

Epizootic - Wide-spread diseases amongst the lower animals.

Extravasation - The passing of blood from the vessels into the surrounding tissues. 
Fermentation-The change which takes place in animal and vegetable substances under heat and moisture.

Fistula - A long, pipe-like track of ulceration, with thickened sides, leading to, or arising from an abscess.

Flatulence - Wind in the bowels.

Fluctuation - The movement of fluid in a cavity, when felt by pressure, or striking with the hand.

Fœtid - Having an offensive smell.

Gangrene - The first stage of mortification.

Glands - Bodies whose office it is to produce or void some special fluid from the blood Thus the liver is a gland, and the bile which it produces assists in digesting the food, etc. The kidney is also a gland, and discharges water from the blood.

Granulations - Grain-like, fleshy bodies which arise in wounds.

Haw - A small body at the inner angle of the eye, which is rapidly drawn across the ball of the eye, to protect it from injury.

Hæmorrhage - Bleeding.

Hereditary Predisposition - A liability to disease transmitted from parent to offspring.

Homœopathy - The word signifies like-suffering, and is used to. designate the new mode of treatment, by the law of healing that "likes are cured by likes."

Hypochondrium - The right and left regions of the liver under the ribs.

Incubation - Breeding. The period between the entrance of poison into the body, and the first appearance of its effects.

Inflammation - A state in which the part, if external, is hot, painful, and swollen.

Interdigital Space - The space between the clefts of the hoof.

Intestinal Canal - The tube from the stomach to the anus.

Invagination - A condition of a portion of the bowel in which one part is received into another, like the involution of the finger of a glove.

Keratitis - Inflammation of the cornea.

Larynx - The upper part of the windpipe.

Lesion - Any hurt, injury, or departure from the healthy condition.

Lumbar Region - The loins. 
Lymphatic Vessels - Vessels for the conveyance of lymph. (See "absorbents.")

Masticate - To chew.

Mucocele - A small swelling, containing mucus and tears, near the inner angle of the eye.

Muco-purulent-A mixture of pus and mucus.

Mucus - The fluid secreted by a mucous membrane, such as that lining the mouth, etc.

Necrosed - Ulcerated bone.

Ophthalmoscope - An instrument used for the illumination of the interior of the eye, whereby diseases can be discovered there.

Osseous - Bony.

Papular - Pimply.

Papule - A pimple.

Paralysis - Loss of motion, or of sensation.

Parasite-A minute animal, or vegetable organism, which feeds upon the body of its host.

Parenchyma - The proper substance of the lungs, liver, etc.

Parturition - The act of delivery.

Pathological - Relating to the investigation of the nature of the disease.

Parotid Gland - A gland for secreting saliva.

Paroxysmal - Occurring in fits.

Pharnyx - The back part of the mouth.

Phlegmonous -- Relating to a tense, painful, circumscribed swelling.

Photophobia - Dread of light.

Plethoric - Full habit of body.

Predisposing Cause-That which renders the body open to disease.

Premonitory-Warning.

Prepuce-Foreskin.

Purulent - Consisting of matter.

Pus - Matter.

Pustular-Mattery.

Pustule - A small elevation of the skin containing matter. 
Rectum - The last portion of the large bowel.

Respiration - The function of breathing.

Respiratory Murmur - The sound caused by the ingress and egress of air, as regards the lungs.

Ruminants - Cud-chewing animals.

Rumination - Chewing the cud.

Salivation - Increased secretion of saliva.

Sanious - Thin, serous, offensive discharge.

Schneiderian Membrane-The mucous membrane lining the nose.

Serous Membrane-A membrane that secretes serum.

Spasm - A muscular contraction.

Suppuration - The process whereby matter is formed.

Symptom - A sign, or mark of disease.

Thoracic Cavity - The cavity of the chest.

Trituration - Reduced to powder.

Traumatic - Belonging to, or caused by wounds.

Trocar - An instrument used for removing the water in dropsy of the chest, belly, etc., and gas from the paunch.

Ulceration - The process by which an ulcer is formed.

Ulcer - A breach on any part of the surface of the body, etc., from which matter is given off.

Ureter - The tube from the kidney to the bladder.

Urethra - The tube from the bladder outwards.

Urinary Passages - The passages for the conveyance of urine.

Uterus - The womb.

Vagina - The passage leading to the womb.

Vertebra - One of the back bones.

Vertigo - Giddiness.

Visceral - Relating to the viscera.

Vesicle - A small elevation of the skin containing clear fluid. 


\section{INDEX.}

Abortion, after treatment, 132

........ in cows, 128

....... in ewes, 404

........ precautions against, 131

....... signs of, 130

Abscess, on coronet, 303

........ on lungs, 181

Aloes, overdose of, 89

Amaurosis, 258

Anchylosis of the pastern, 294

Apoplexy, 237

........ splenic, 112

........ treatment of, 241

Appetite, loss of, 75

Applications, list of local, 448

Aptha, $\cdot 37$

........ epizootic, 47

Ascarides, 78

Ascares lumbricoides, 77

....... megalocephala, 77

Ascites, 104

Astragalus, 376

Back, sprain of, 297

Back raking, 87

Back tendons, sprain of, 299

........ thickening of, 299

Bellyache, in horses, 84

... .. in other animals, 86

Belly, dropsy of, 104

Bile, 334

Black Quarter, 282

...... Leg, 282 ।

Bladder, inflammation of, 124

...... stone in, 126

Blain, in oxen and sheep, 44

...... in pigs, 45
Bleeding from the lungs, 181

...... case of, 182

...... from the nose, 147

Blood diseases, 280

Bloody flux, in horses, 93

...... in oxen, 94

...... sheep and pigs, 95

Bloody urine, in horses, 122

...... in oxen, 122

Blowfly, 260

Blue disease, 284

Bone spavin, 292, 377

Bone wen in chickens, 443

Bony enlargements, 292

Bots, in horses, 79

...... in sheep, 156

Bowel, intussusception of, 103

...... sickness in sheep, 93

Bowels, confined, 82

...... diseases of the, 77

...... earthy stones in, 81

...... inflammation of, 96

...... loose, 88

...... twists in, 103

...... worms in, 77

Brain, concussion of the, 245

...... fever, 240

..... hydatids of the, 246

..... inflammation of, 240

...... water on the, 245

Braxy in sheep, 92, 281

Breeding of pigs, 416

Breeds of sheep, 393

Broken down suspensory ligament, 299

....... knees, 287 
Broken ribs, 370

....... wind, 186

Bronchial tubes, inflammation of, 164

Bronchitis, 164

Broncho-Pneumonia, 179

Bruises of the leg and foot, 302

Brushing, 302

Bursal enlargements, 295

Calculi, 126

Calf, scour in, 90

...... slipping the, 130

Calves, earliest treatment of, 138

Calving, dropping after, 325

Canine madness, 224

Cannon bone, 381

Capped elbow, 296

...... hocks, 296

Carbuncular Diseases, 280

$\ldots \ldots$ in pig, 59, 284

Caries, 34

Case of Abscess, 181

..... bleeding from the lungs, 182

...... bronchitis, 166

..... broncho-pneumonia, 179

...... catarrh in pony, 150

....... chronic cough, 185

...... colic, 88

....... congestion of lungs, 163

...... diabetes, 119

...... diarrhœea, in horse, 89

...... diarrhœa in cow, 90

...... double pneumonia and pleurisy, 175

dysuria 127

...... garget, 144

..... grease, 272

...... hysteria, 231, 232

...... impetigo, 272

Case of pleurisy, 175, 176, 178

...... pleuro-pneumonia, 176

...... purpura hemorrhagica, 321 , 322,323

..... retained placenta, 138

...... rheumatism, $315,316,317$

..... ringbone, 295

...... sore throat, 56, 57

...... spasm of the diaphragm, 215

...... spavin, 294

...... sprain, 300, 301, 302

...... typhoid pneumonia, 177

Cataract, 257

Catarrh, nasal, 148

...... epizootic, 157

Catarrhal fever, 157

Cattle plague, 347

...... treatment of, 351

...... American, 355

...... treatment of, 363

Cause of splint, 384

Cellutitis, 337

Chal sbeate infusions, 129

Chapped teats, 267

Chest, dropsy of, 182

...... founder, 314

Chest, tapping the, 183

Chickens, diseases of, 442

...... general management of, 424

Chine felon, 314

Chip or chipping in poultry, 443

Choking, in horses, 60 in oxen and sheep, 61

Cholera, hog, 284

Chronic cough, 183, 197

...... inflammation of the joints, 391

Clap, 141

rheumatism, 314

Cleansing, the, 137

$\ldots \ldots$. indigestion in the cow, 69 Coffin joints, 294

...... inflammation of the lungs, Colic, flatulent, 85 171

...... case of, 88 inflammation of the pleura, 176

megrims, 234

...... milk fever, 332

...... myalgia, 319

..... nasal gleet, 152

...... pericarditis, 222

$$
\text { case of, } 88
$$$$
\text { in horses, } 84
$$

$\ldots \ldots$ in other animals, 86

Collections of pus in the nose, 153

...... matter in guttural pouches, 154

Concretions in bowels, 81

Concussion of the brain, 245 
Conformation of the hock, 375 ...... of the leg, 383

Congestion of the lungs, 162 ...... case of, 163

Contraction of the hoof, 388

Core in poultry, 443

Corns, 389

Coryza, in horses, 148

...... in oxen, malignant, 149

Costiveness, 82

...... in poultry, 443

Cough, broken winded, 194

...... bronchial, 192

...... chronic, 183,197

...... classification of, 190

...... consumptive, 197

...... from dentition, 191

$\ldots \ldots$ in general, 188

...... laryngeal, 191

...... pharyngeal, 191

..... remedies for, 199

...... pulmonary, 193

...... tracheal, 192

Coup de soleil, 238

Cow-hocked, 299

Cow-pox, 366

Cracked heels, 267

Crammed stomach, 72

Cramp of leg, 298

Crib biting, 74

Cuneiform magnum, medium, parvum, 376

Curb, 298

Curby hocked, 299

Cutting and brushing, 302

Cystitis, 124

Delirium, 239

Delivery, mechanical means for assisting, 136

...... flooding after, 136

Dentition, in horses, 29

...... in oxen, 31

$\ldots \ldots$ in sheep, 31,413

..... in pigs, 32

Dermatodectes equi, 262

...... ovis, 263

Diabetes, case of, 119

...... insipidus, 117

....... mellitus, 118

Diaphragm, spasm of, 215
Diarrhœa, case of, 89

...... in horses, 88

...... oxen and sheep, 90

..... pigs, 91

Diet in sickness, 28

Difficulty in urinating, 127

Dilatation and stricture, 63

Dilated heart, 220

Directions for compounding medicines, 453

Discharge from the vagina, 141

Disease, blue, 284

...... navicular, 342

D.... of the horns, 391

Diseases, blood, 281

...... carbuncular, 281

...... of the bladder, 115

...... of the bowels, 77

...... eye, 251

..... generative organs, 128

..... gullet, 60

..... heart, 218

...... kidneys, 115

...... liver, 107

...... mouth, 37

...... nervous system, 224

..... not classified, 305

...... parasitic, 260

..... of poultry, 442

...... of the respiratory organs 147

...... skin, 260

..... spleen, 107

...... stomach, 64

..... teeth, 29

...... throat, 54

Disinfectants, 365

Dislocation of patella, 298 ?

Distemper in pigs, 284

...... in sheep, 281

Distoma hepaticum, 111

Docking, 409

Dose, how much to give, 25

...... how often to give, 26

Dropping after calving, 325 .

Dropsy of the belly, 104

...... of the chest, 182

Ducks and geese, 435

Dust and hair balls, 81

Dysentery, in horses, 98 
Dysentery, in oxen, 94

$\ldots \ldots$ in sheep and pigs, 95

Dysuria, case of, 127

Early treatment of calves, 138

...... of lambs, 407

Echinorynchus gigas, 78

Eczema, 47

Elbow, capped, 296

Enlargements, bony, 292

..... bursal, 295

Enteritis, 96

...... with exudation, 98

Epizootic aptha, 47

...... catarrh, 157

..... eczema, 47

Eruptions, erythematous, 266

...... papular, 269

...... pustular, 270

Eruptive fevers, 274

Erysipelas, 274

Erythema, 267

Erythematous Eruptions, 266

Evil, wood, 102

Ewe, her pregnancy, etc., 402

...... parturition, 404

Ewe's milk, use of, 412

Examination of the patient, 19

External applications, list of, 448

Eye, diseases of the, 251

...... injuries to, 251

Face, fistula of the, 35

Facial paralysis, 248

Farcy, 308

Farcy buds, 309

Fardel bound, 71

Feeding of poultry, 433

Festered horn, 391

Fever, brain, 240

Fever, catarrhal, 157

...... inflammatory, 282

...... milk, 325

...... puerperal, 325

Fevers, eruptive, 274

Fistula, in poll evil, 291

...... of the coronet, 304

...... of the face, 35

Fistulous withers, 292

Flatulent colic, 85

Flooding after delivery, 136

Flukes in the liver, 111 .
Flux, bloody, 93

....... in oxen, 94

$\ldots \ldots$ in sheep and pigs, 95

Foals, costiveness in, 83

Foot and mouth disease, 47

Foot rot in sheep, 337

...... malignant, 339

...... treatment of, 340

Foreskin, stone in, 126

Fractures, 368

...... of the horn, 374

...... of the jaws, 371

...... of the leg, 373

...... of the nasal bones, 371

...... of the pastern bones, 370

...... of the pelvis, 369

...... of the ribs, 370

..... of the shoulder blade, 372

...... tibia, partial, 372

...... treatment of, 372

Frenzy, 240

Fumigations, 451

Gadfly, 79, 261

Gall-bladder, 334

Gaps in chickens, 443

Garget, 142

....... case of, 144

Gas in the paunch, 65

Geese, 435

General management of sheep, 401

...... remarks on nasal discharges, 155

Generative organs, diseases of, 128

Gid of sheep, 235

Giddiness, 232

...... treatment of, 241

Glanders, 306

..... ox, 149

Gleet, nasal, 151

Gloss anthrax in oxen and sheep, 44,281

...... in pigs, 45

Glossary, 460

Gonorrhœea, 141

Grass staggers, 71

Grapy grease, 271

Grease, 270

...... case of, 272

Gripes, 84

Gullet, diseases of, 60 
Gullet, obstruction of, 61

...... stricture of, 63

Gutta serena, 258

Guttural pouches, collection matter in, 154

...... inflammation of, 55

Hair balls, 81

Hatching of chickens, 430

Haw, 254

Heart, dilated, 220

...... diseases of, 218

...... enlargement of, 219

...... palpitation of, 218

Heels, cracked, 267

Hematuria, 122

Hemiplegia, 248

Hemorrhoids, 103

Hepatitis in horses, 108

$\ldots \ldots$ in oxen, sheep, etc., 109

Hide bound, 67

Hind legs paralyzed, 230

Hip down, 369

..... sprain of, 298

Hock, conformation of the, 375

Hocks, capped, 296

Hog cholera, 284

Hollow horn, 391

Hoof, contraction of, 388

Hoose, 216

Horns, broken, 374

...... disease of the, 391

Horse bot, 79

...... shoe, patent, 344

Hove, 65

...... relapsing, 66

Hydatids of the brain, 246

Hydrophobia, 224

Hydrothorax, 182

Hysteria, 230

...... cases of, 231

Impaction of stomach, 72

Impetigo, 270

Importance of splints, 386

Incised wounds, 286

Indigestion, 67

...... acute, 85

Inflamed throat, 54

Inflammation of the bladder, 124

...... of the bowels, 96

....... brain, 240
Inflammation of the bronchial tubes, 164

...... guttural pouches, 55

of ...... jaw bone, 35

....... joints, 391

...... kidneys, 115

...... liver, 108

...... lungs, 167

...... lymphatics, 335

...... mouth, 38

...... mucous membrane of bowel, 93

...... nose, 148

...... parotid gland, 52

...... peritoneum, 99, 104

..... pleura, 172

...... prepuce. 142

..... serous membrane of bowel, 99

tongue, 38

$\ldots$.... udder, 325

Inflammatory fever, 282

Influenza, 157

Inhalations, 450, 451

Injuries, 286

..... about the mouth and tongue, 41

Insects in the brain, in cows, 247

...... in sheep, 246

Intestinal worms, in horses, oxen, and sheep, 77

...... in pigs, 78

Introductory remarks, 19

Intussusception of bowel, 103

Itch, 262

Jaundice, 107

Jaw joint open, 53

....... osteo-sarcoma of, 43

Jaw, fractures of, 371

...... inflammation of, 35

Joint felon, 314

...... murrain, 282

...... coffin, 294

...... open, 289

Kidneys, diseases of, 115

...... inflammation of, 115

...... stone in, 126

Killing of pigs, 418

Knees, broken, 287 
Lambs, early treatment of, 407

...... weaning of, 410

Lameness, groggy, 342

Lampas, 42

Leucorrhœa, 141

Leg bones, fracture of, 373

Lice, 261

Lichen, 269

List of external applications, 448 ...... internal remedies, $\mathbf{4 4 6}$

Liver, diseases of, 107

...... flukes in the, 111

..... inflammation of, 108

...... of the $\mathrm{ox}, 333$

Lockjaw, 226

Loin bound, 314

Long wool sheep, 398

Looseness of bowels in horses, 88

..... case of, 89

..... in oxen and sheep, 90

...... case of, 90

...... pigs, 91

Loss of appetite, 75

Lotions, $\mathbf{4 4 8}$

Lumbago, 314

...... case of, 315

Lumps in the udder, 143

Lung parasites, 216

Lungs, abscess in, 181

...... case of, 181

...... bleeding from the, 181

..... case of, 182

...... congestion of, 162

...... case of, 163

..... inflammation of, 167

...... parasites in the, 216

Lymphatitis, 335

Madness, canine, 224

Mad staggers, 70,239

...... treatment of, 241

Maggots on sheep, 260

Malignant coryza, 149

...... pustule, 280

Mallenders, 273

Mange, 262

..... from diseased straw, 266

Measles, 275

Mechanical means for assisting delivery, 136

Megrims, 232
Membranes, synovial, 390

Merino sheep, 394

Mercurial poisoning, 43

Metacarpal bone, 381

Metritis, 140

Middle wools, 396

Milk fever, 325

...... cases of, 332

..... treatment of, 329

...... general directions for, 331

Molten grease, 93

Moon blindness, 253

Moor ill, 102

Mouth and foot disease in oxen, 47

$\ldots \ldots$ in sheep and pigs, 50

Mouth, inflammation of, 38

...... injuries about, 41

...... ulceration of the, 339

Mumps, 52

Murrain, 47, 347

Myalgia, 318

Nasal bones, fracture of, 370 catarrh, 148

...... discharges, general remarks on, 155 gleet, 151

Navicular disease, 342

Neck, sprain of, 297

Nephritis, 115

Nests for sitting hens, 426

Nettlerash, 268

Nodes, 292

Nose, bleeding from, 147

...... collection of pus in, 153

...... sheep bots in, 156

Nursing, directions for, 27

Obstruction of the gullet, 60

Estrus equi, 79

..... hemorrboidalis, 79

...... veterinus, 79

Ophthalmia, 251

...... periodic or specific, 253

Open joint, 289

Os calcis, 376

Osteo sarcoma of jaw, 43

Overreach, 303

Ox glanders, 149

Pain in stomach, 75

Palpitation of the heart, 218

Papular eruptions, 269 
Paralysis, facial, 248

.... of the hind half, 249

..... of the legs, 230

....... of the side, 248

....... tongue, 40

Paraplegia, 249

Parasites in the lungs, of sheep, 216 ...... of calves, 216

...... nose of sheep, 156

...... vegetable, 266

Parasitic diseases, 260

Parotid gland, inflammation of, 52

Partial fracture of the tibia, 372

Parturition, in cows, 132

...... in ewes, 404

Pastern bones, 370

Patella dislocated, 298

Patent shoe, 344

Patient, examination of, 19

Paunching, 73

Pelvis bone, fracture of, 369

..... sprain of, 298

Pericarditis, 220

Periodic ophthalmia, 253

Peritoneum, inflammation of, 99

Peritonitis, 99

Phthiriasis, 262

Phytolacea decandra, 144

Pigs, breeding, rearing, etc., 416

..... carbuncular disease in, 59 , 284

$\ldots \ldots$ killing of, 418

Piles, 103

Pilules, 24

Placenta, retained, 137

case of, 138

Pleura, inflammation of, 172

Pleurisy, 172

Pleurodynia, 314

Pleuro-pneumonia in cattle, 208

...... treatment of, 212

$\ldots \ldots$ in horses, cases of, 176,178

Pneumonia, broncho, case of, 179

..... pleuro, in cattle, 208

..... in horses, 167

...... case of, 175

..... pleuro, case of, 178

...... typhoid, case of, 177

Poisoning, antidote for, 459
Poisoning, mercurial, 43

Pokeweed, 145

Poll evil, 290

Polypus in gullet, 60

Poultry, care of eggs, 427

...... different breeds of, 419

...... diseases of, 442

..... feeding for market, 433

...... general management of, 423

...... hatching of, 430

Premonitory signs of Abortion, 130

..... pneumonia in cattle, 208

Prepuce, inflammation of, 142

Profuse staling, 117

Protrusion of the rectum, 105

...... tongue, 40

Prurigo, 269

Psoriasis, 273

Puerperal fever, 325

Pulse, in horse, 20

..... in mule, 20

..... in ox, 20

...... in sheep, 20

...... where to feel the, 20

Purpura hœmorrhagica, 320

Pus in nose, collection of, 153

Pustular eruptions, 270

Pustule, malignant, 280

Putrid sore throat, 58

Quarter evil, 282

Quittor, 303

Rabies, 224

Ranula, 43

Rectum, eversion of, 105

...... protrusion of, 105

Red water, 123

Remarks, introductory, 19

Remedies, list of, 446

..... selection of, 23

...... the, 24

Respiration, frequency of, 21

Respiratory organs, diseases of, 147

Retained placenta, 137

...... case of, 138

Retching, 64

Retention of urine, 120

Rheumatism, acute, 813

...... chronic, 314

..... in cow, case of, 315

...... in horse, case of, 317 
Ribs, broken, 370

Rinderpest, American, 355

.......treatment of, 363

..... Great Britain, 347

....... treatment of, 351

Ringbone, 293, 386

...... case of, 295

...... recipes for treatment, 454

Ringworm, 266

Roaring, 186

Round worms, 77

Rot, in oxen and sheep, 111

Roup, in chickens, 443

Ruptured stomach, 71

Saddlegall, 267

Salivation, 43

Sallenders, 273

Sarcoptes equi, 262

...... ovis, 263

Scabies, 262

Scab in sheep, 263

Scanty urine, 119

Scarlatina, 276

Scoke, 144

Scour in calves and lambs, 90

..... in chickens, 443

Shearing, 416

Sheep, bots in nose of, 156

...... breeds of, 393

..... breeds of, 393
...... general mang wool, 398

...... middle wool, 396

Sheep's skin, 413

Shoulder blade, fracture of, 372

...... sprain of, 298

Shoulder tied, 314

Sitfast, 267

Sidebone, 293

Skin, diseases of, 260

...... parasites on, 260

Sleepy staggers, 69, 239

Slipping the calf, 128

Small-pox, 277

Soreness and stiffness, 318

Sore teats, 145 throat, 54

Spanish fever, 355 sheep, 394

Spasm of diaphragm, 215 ...... leg, 298
Spavin, 292, 377

...... detectable, 379

...... occult, 379

...... recipes for treatment, 454

Speedy cut, 302

Spleen, diseases of, 107

Splenic apoplexy, 112, 281

Splint, 293, 381

...... cause of, 384

...... recipes for treatment of 454

Sprains of the back, 297

... . back tendons, 299

...... hip, 298

..... neck, 297

..... pelvis bone, 298

...... shoulder, 298

..... stifle, 298

Staggers, sleepy, 69, 239

..... mad, 70, 239

Staling, difficult, 127

...... profuse, 117

...... scanty, 119

Stifle, sprain of, 298

Stomacace, 359

Stomach, crammed, 72 diseases of the, 64

....... pain in the horse, 75

...... ruptured, 71

..... staggers in horses, 69,239

..... staggers in oxen, 71

...... treatment of, 241

Stones, in bladder, 126

...... bowels, 81

...... foreskin, 126

...... kidneys, 126

...... prepuce, 126

...... ureter, 126

..... uretha, 126

Strain of the flexor tendon, 342

Strangles, 161

Strangulation of the bowel, 103

Stricture, 63

Stringhalt, 250

Strongylus armatus, 77

...... radiatus, 77

Stumbling, 388

Sturdy, 235

Sunstroke, 238

...... treatment of, 241

Surfeit, 268 
Symbiotes equi, 262

Synovial meinbranes, 390

Sweeny, 305

Toenia denticula, 77

...... expansa, 77

...... mamilana, 77

...... perfoliata, 77

..... plicata, 77

Tape worm, 77

Tapping the chest, 183

Teats, chapped, 267

...... sore, 145

Teeth, as tests of age, in horses, 29

...... in oxen, 31

...... in pigs, 32

...... in sheep, 31,413

...... decayed, 34

..... diseases of the, 29

...... general symptoms eased, 36

...... irregularities of, 32

Teething, difficult, 33

Tendons, back, sprain of, 299

Tetanus, 226

Texas cattle disease, 355

Thick wind, 186

Thorough pin, 295

Thread worm, 77

Throat diseases of the pig, 59

..... diseases of the, 54

...... inflamed, 54

..... obstruction in, 61

...... polypus in, 61

..... putrid or nualignant sore, 58

...... sore, 54

Thrush of the feet, 345

...... of the mouth, 37

Tibia, fracture of, 372

Ticks, 261

Tinctures, 24

..... list of, 446

Tongue, inflammation of, 38

..... injuries to, 39,41

...... protrusion of, 40

Toothache, 35

Tooth, " buck," 312

...... "woll's," 33

Tread, 303

Trichiasis, 252

Triturations, 24
Triturations, list of 446

Turkeys, rearing of the chicks, 440

Turnsick, 246

Twists, etc., of the bowels, 103

Typhoid pneumonia, case of, 177

Udder, inflammation of the, 142

Ulceration of the mouth, 47, 339

...... of the feet, 337

Ureter, stone in, 126

Uretha, stone in, 126

Urinating, difficulty of, 127 .

Urine, bloody, 122

...... incontinence of, 121

...... retention of, 120

...... scanty, 119

Urticaria, 268

Use of ewe's milk 412

Vagina, discharge from, 141

of dis- Variola, 277

Vegetable parasites, 266

Vertigo, 232

Vomiting, 64

Warbles on skin, 261

Warts, 272

Water farcy, 310

...... on the brain, 245

..... red, in cattle, 123

Weaning of calves, 139

...... of lambs, 410

Weed, 335

Wheezing, 186

Whistling, 187

Whites, the, 141

White scour, in calves and lambs, 90

Wind, broken, 186

...... thick, 186

Windgalls, 295

Wind sucking. 74

Windy colic, 85

Withers fistulous, 292

Woinb, inflammation of, 140

Wood evil, 102

Wool and its peculiarities, 399

Wools, long, 398

...... middle, 396

Worms, intestinal, 77

Wounds and injuries, 286

Y..... incised, 286

Yellows, the, 107 


\section{ILLUSTRATIONS.}

I Plate I.-Front view of the bones of the knee . . 289

Plate II.-Showing external diseases of the horse . 290

Plate III.-Tendons and ligaments of the fore leg . 298 Plate IV.-Devon cow . . . . . . . . . . . . 325

Plate V.-Metacarpal and sesamoid bones. . . . . 336 Plate VI.-Devon Ox . . . . . . . . . . . . 355 Plate VII.-Skeleton of the horse . . . . . . . 386 







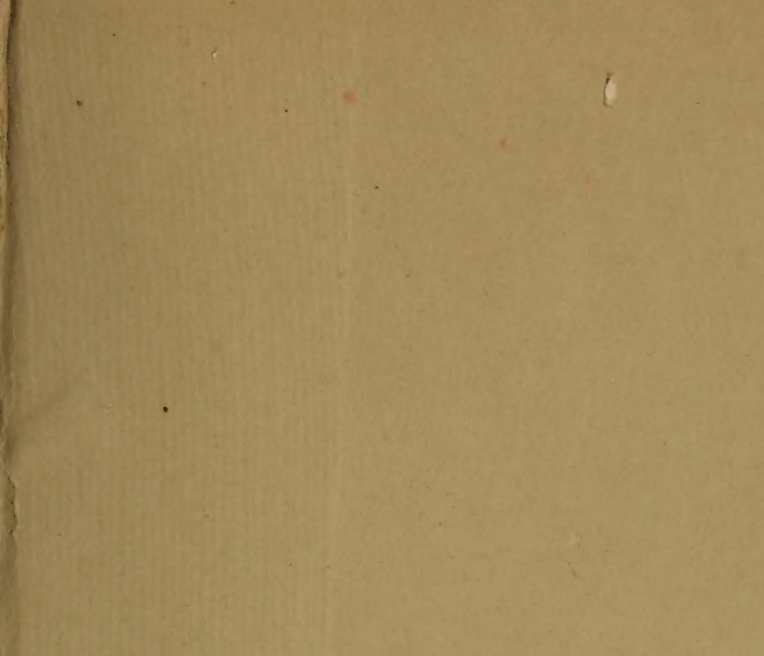

1.
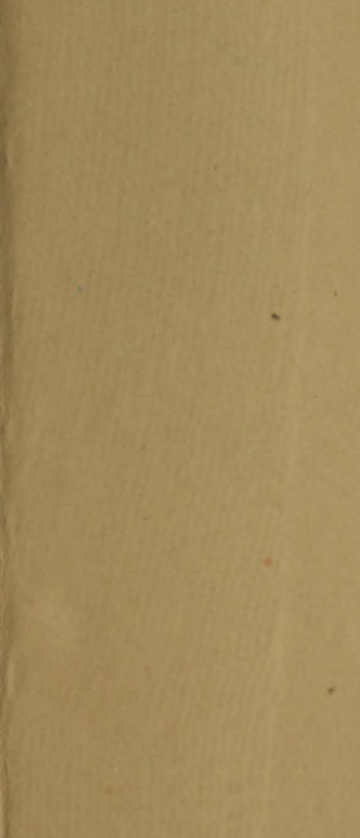

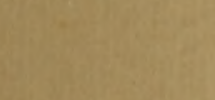
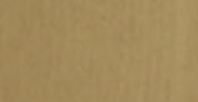


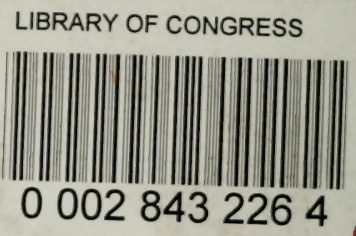

